\title{
Thin Layer Flow in \\ Rolling Element Bearings
}
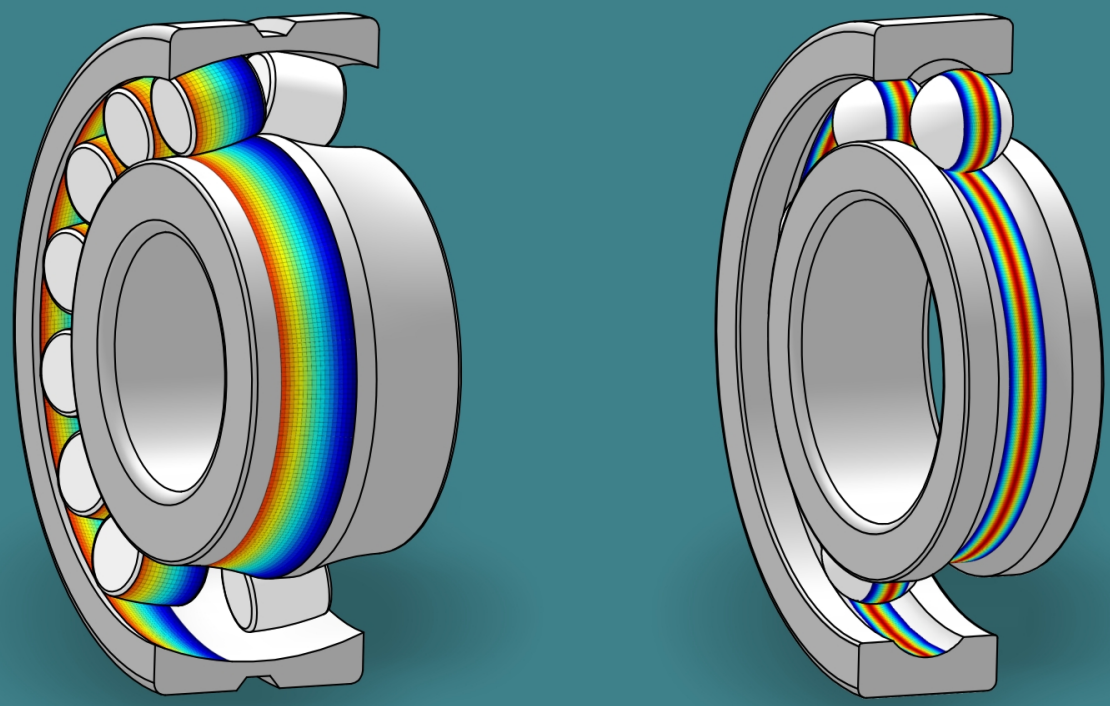

M.T. van Zoelen 



\section{Thin Layer Flow in Rolling Element Bearings}

M.T. van Zoelen 
This research project was supported by SKF Engineering \& Research Centre in Nieuwegein, the Netherlands, and carried out at the University of Twente, the Netherlands. The support is gratefully acknowledged.

Thin Layer Flow in Rolling Element Bearings M.T. van Zoelen

Cover: M.T. van Zoelen

Thesis University of Twente, Enschede - With a summary in Dutch. ISBN 978-90-365-2934-1

Copyright (C 2009 by M.T. van Zoelen, the Netherlands 


\section{THIN LAYER FLOW IN ROLLING ELEMENT BEARINGS}

\section{PROEFSCHIFT}

ter verkrijging van

de graad van doctor aan de Universiteit Twente, op gezag van de rector magnificus, prof. dr. H. Brinksma, volgens besluit van het College voor Promoties

in het openbaar te verdedigen op vrijdag 11 december 2009 om 16.45 uur

door

Marco Theodorus van Zoelen

Geboren op 27 augustus 1981

te Apeldoorn 
Dit proefschrift is goedgekeurd door de promotoren:

prof. dr. ir. H.W.M. Hoeijmakers

prof. dr. ir. P.M. Lugt

en de assistent promotor:

dr. ir. C.H. Venner 


\section{TABLE OF CONTENTS}

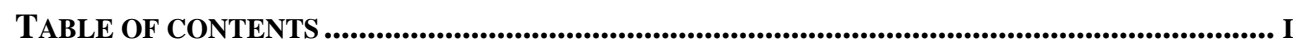

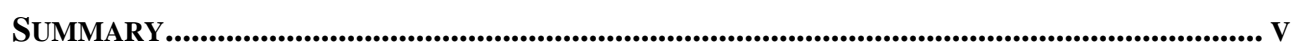

SAMENVATTING ......................................................................................................................... VII

NOMENCLATURE................................................................................................................ IX

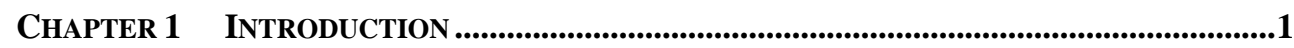

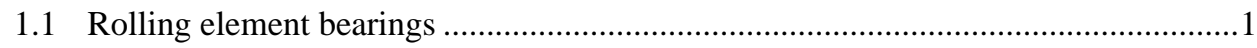

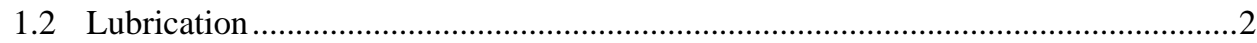

1.2.1 Elasto - Hydrodynamic Lubrication ...............................................................

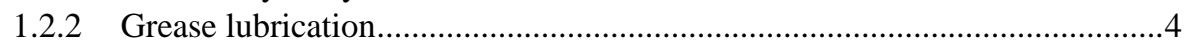

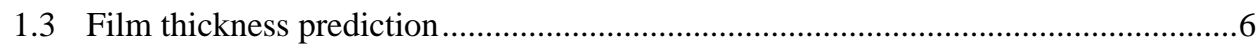

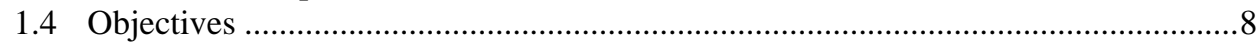

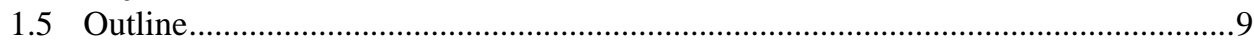

CHAPTER 2 CENTRIFUGAL EFFECTS ON FLOW …....................................................11

2.1 Thin layer flow modeling ..................................................................................11

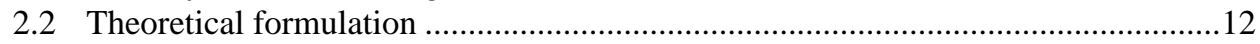

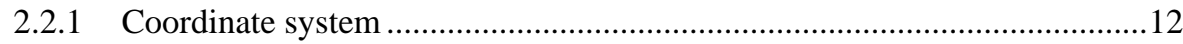

2.2.2 Equations of motion .................................................................................

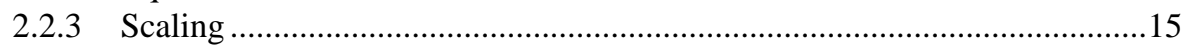

2.2.4 Boundary conditions.....................................................................................16

2.2.5 Scaled body force, pressure and flow velocity..............................................18

2.2.6 Layer thickness equation .........................................................................19

2.2.7 Simplification and analytical approach .......................................................20

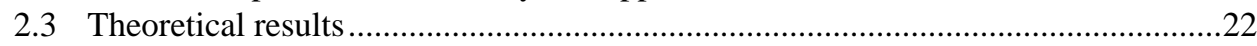

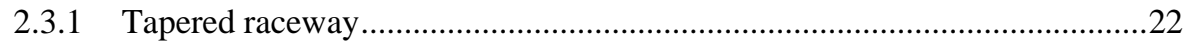

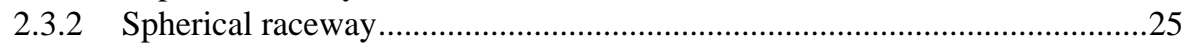

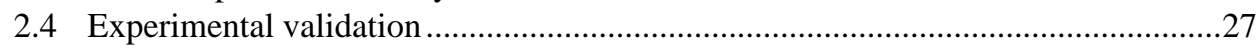

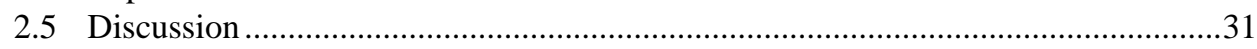

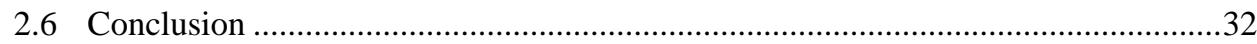

CHAPTER 3 CENTRIFUGAL EFFECTS IN ROLLING ELEMENT BEARINGS...........................33

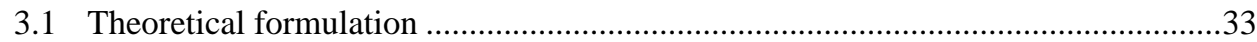

3.1.1 Layer thickness equation ...........................................................................34

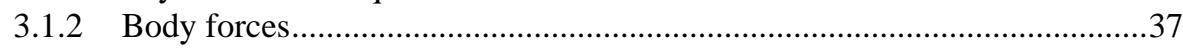

3.1.3 Solution layer thickness equation..................................................................39

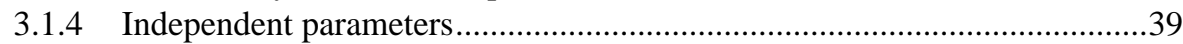

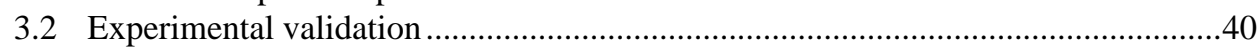

3.2.1 Experimental setup and procedure …………………………….................40 
3.2.2 Experimental results ........................................................................... 42

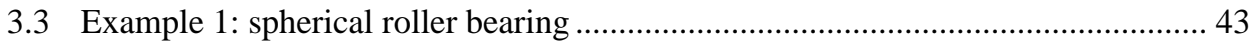

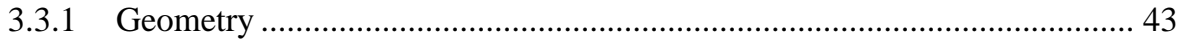

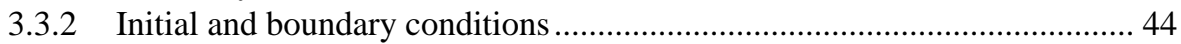

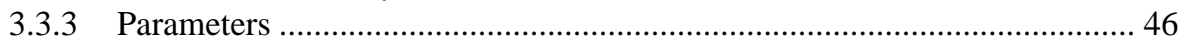

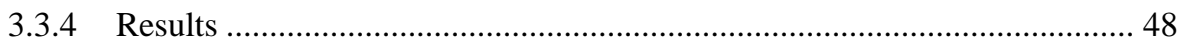

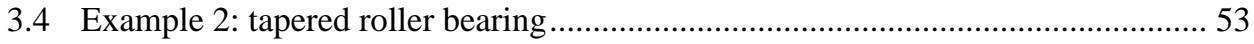

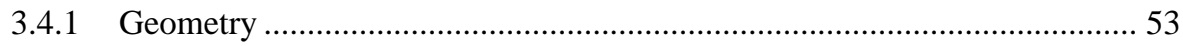

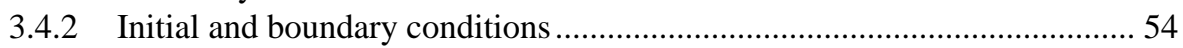

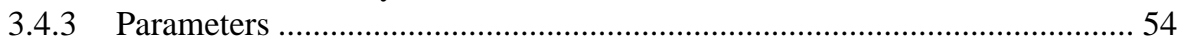

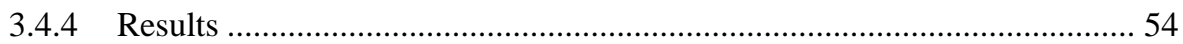

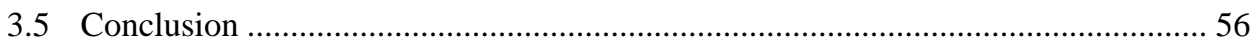

CHAPTER 4 ELASTO-HYDRODYNAMIC LUBRICATION ................................................57

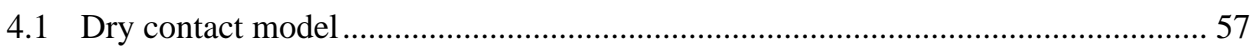

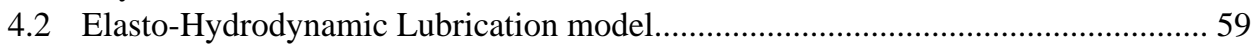

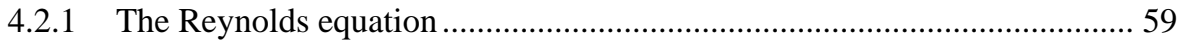

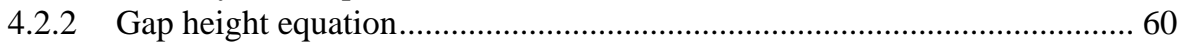

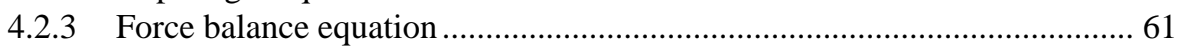

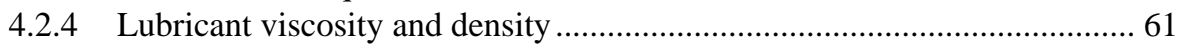

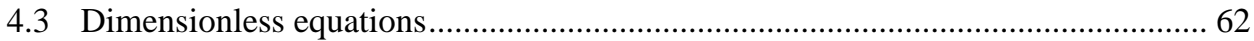

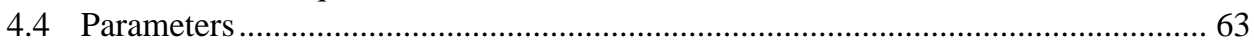

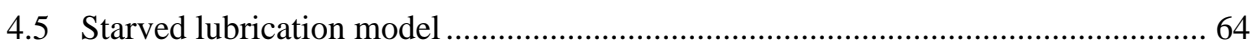

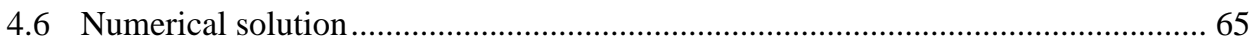

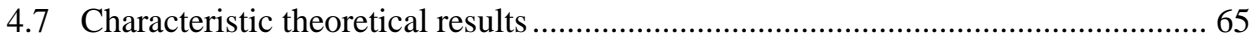

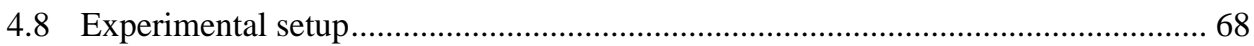

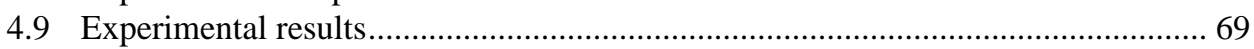

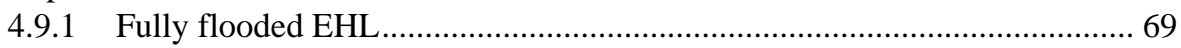

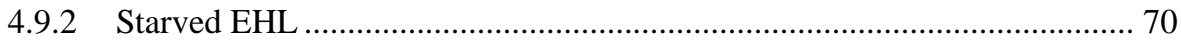

CHAPTER 5 FILM THICKNESS DECAY IN STARVED EHL CONTACTS ...............................73

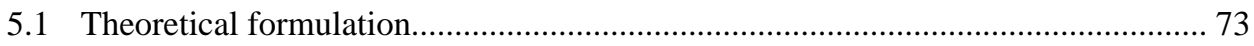

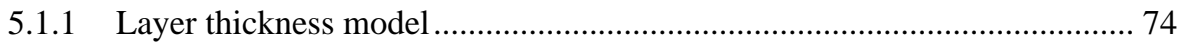

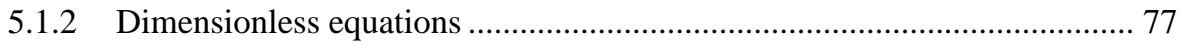

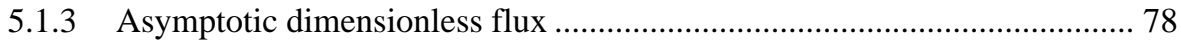

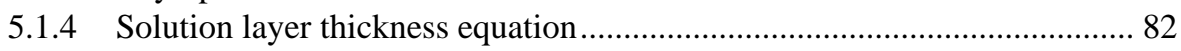

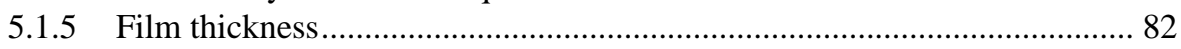

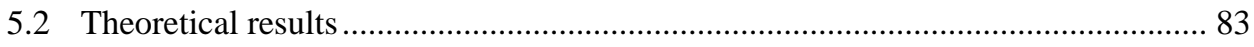

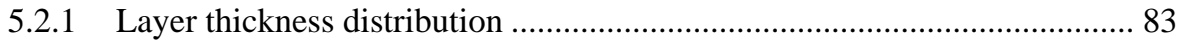

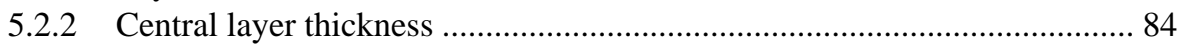

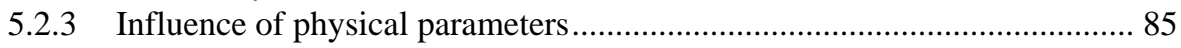

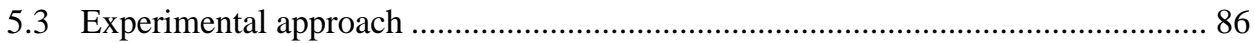

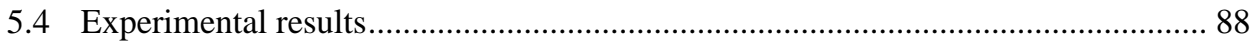

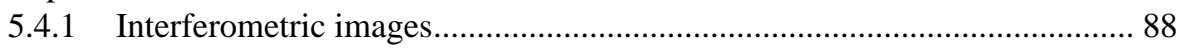

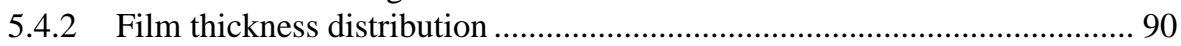

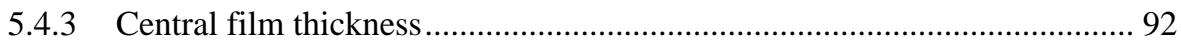

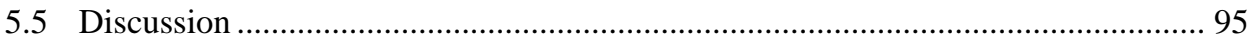


5.5.1 Film thickness decay model Chevalier/Damiens ........................................95

5.5.2 Centrifugal effects and contact pressure effects.......................................96

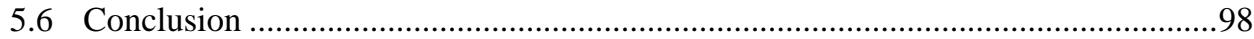

CHAPTER 6 LAYER THICKNESS DECAY IN RADIALLY LOADED BEARINGS .....................99

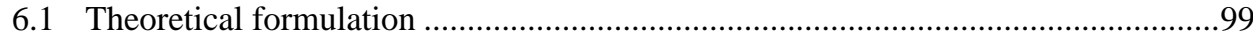

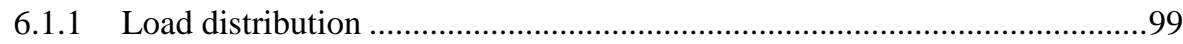

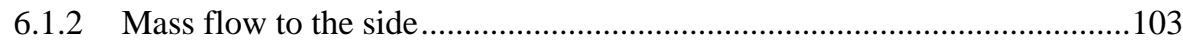

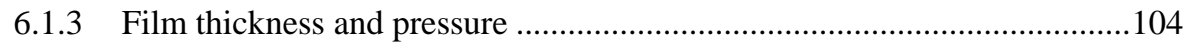

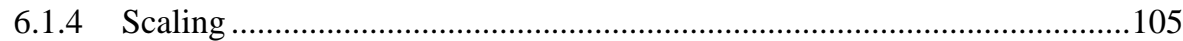

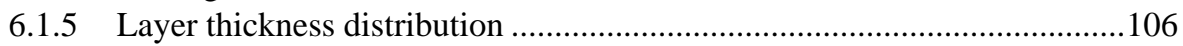

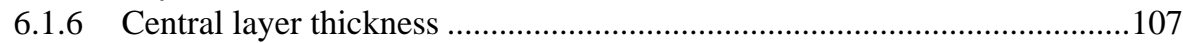

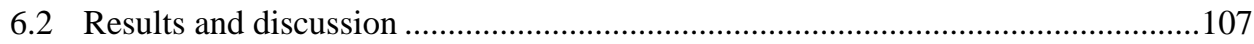

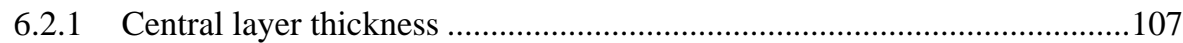

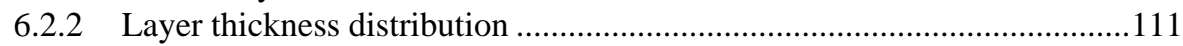

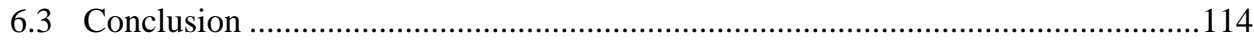

CHAPTER 7 CONCLUSION AND RECOMMENDATIONS...............................................115

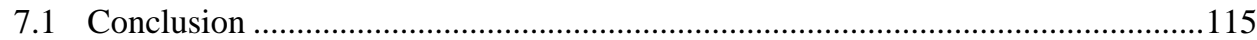

7.2 Recommendations for future research .............................................................116

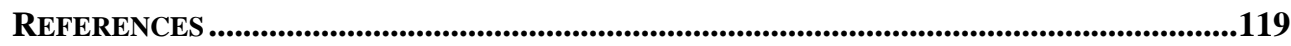

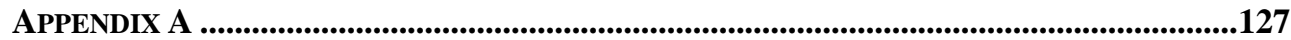

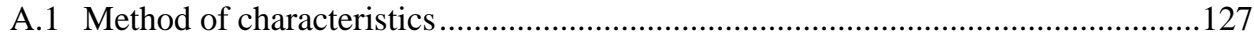

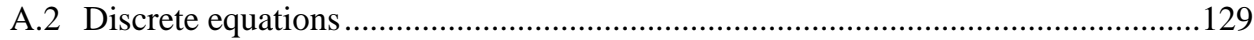

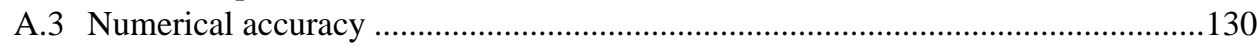

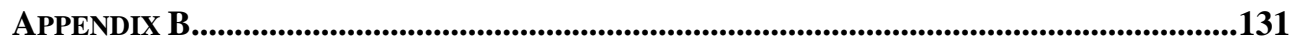

B.1 Coordinate transformations............................................................................... 131

B.2 Acceleration vector of a fluid particle ............................................................132

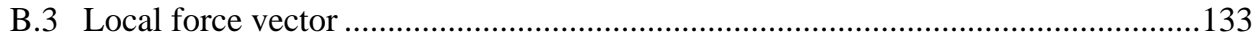

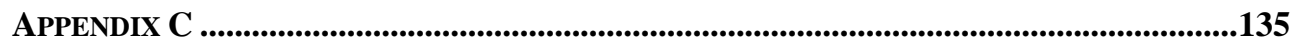

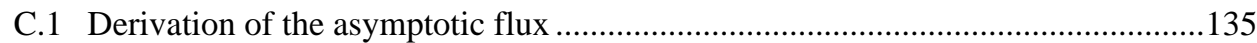

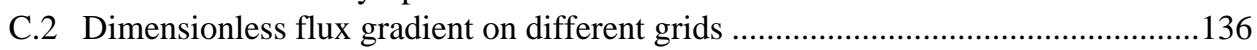

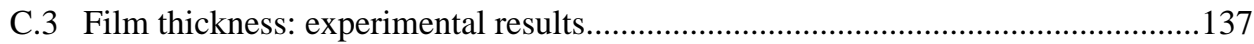

C.4 Details implementation Chevalier/Damiens model ...........................................139

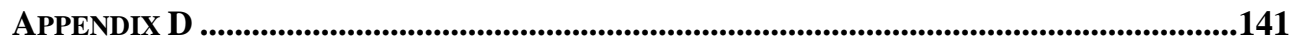

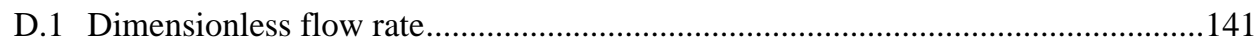

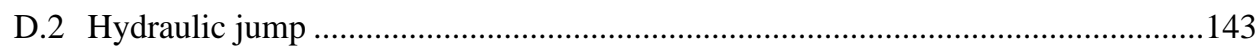

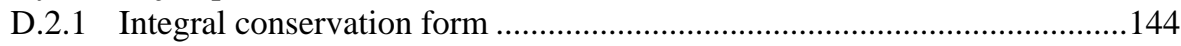

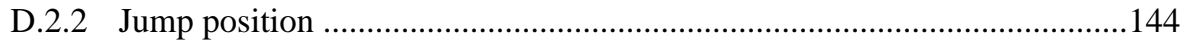

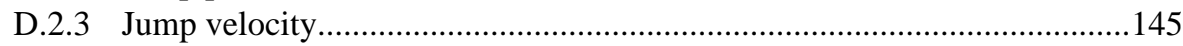

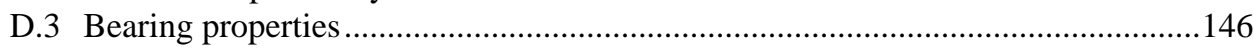

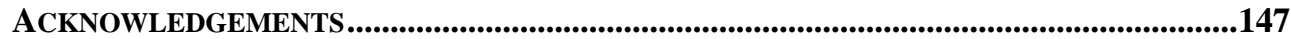

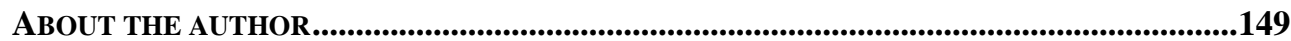




\section{SUMMARY}

To extend the service life of rolling element bearings lubricant such as oil or grease is used. The lubricant significantly reduces the wear of the steel surfaces, provided that the lubricant is able to form a sufficiently thick film to separate the surfaces in the contacts between the different parts in the bearing. In the lubricated contacts between the rollers and the raceways pressures of several GPa can occur. In that case the elastic deformation of the rollers and the raceways has a significant effect on the film thickness and shape. Such contacts are referred to as Elasto-Hydrodynamically Lubricated (EHL) contacts. When the thickness of the lubricant film in an EHL contact is reduced due to insufficient supply of lubricant, it is operating in the so-called starved lubrication regime. This typically occurs in grease lubricated rolling element bearings. As most bearings are lubricated with grease, accurate prediction of the EHL film thickness in starved lubricated rolling element bearings is of great importance.

The film thickness in a starved EHL contact is directly related to the thickness of the lubricant layers on the running tracks supplied to the contact. In rolling element bearings the thickness of these supply layers is determined by many effects. Accurate models predicting the influence of these effects on the thickness of these supply layers are a prerequisite to the reliable prediction of the film thickness in rolling element bearings operating in the starved regime.

In this thesis two effects that influence the supply layer thickness in rolling element bearings are studied in detail.

First the effect of the centrifugal forces on the supply layer thickness is considered. As a first step the layer thickness on the raceway of the inner ring is analyzed separately. Therefore, a free surface thin layer flow equation for axisymmetric rotating surfaces is derived. For the case of bearing applications it is shown that a quasi-linear differential equation can be derived for the layer thickness, as a function of location and time. Experiments have been carried out using real bearing raceways. The results showed good agreement with the model predictions.

The model for the raceway is used to obtain a model for the prediction of the change of the layer thickness due to centrifugal effects in rolling element bearings. In particular, the model is used to predict the layer thickness on the surface of the inner and outer raceway and each of the rollers. In this extended model it is assumed that the lubricant layers in each of the roller raceway contacts separate equally between the diverging surfaces. To provide some justification for this hypothesis, a roller/plate experiment has been carried out.

As an example, the model is applied for the geometry of a spherical roller bearing and a tapered roller bearing. Depending on the shape of the lubricated surfaces, two flow types 
are distinguished for the spherical roller bearing. A similar decrease of the central layer thickness is predicted for various sizes and for different geometry parameter settings. It is shown that the centrifugal effects can significantly reduce the layer thickness, within the service life of the bearing.

Secondly, the effect of the flow in circular or elliptical starved EHL contacts on the supply layer thickness is studied. It is assumed that the reflow or supply of new lubricant to the track is negligible. The model is validated experimentally using optical interferometry. It is found that for severely starved contacts the film thickness decay rate is smaller at higher loads and it is independent of the velocity. Also, in most cases the flow in the EHL contacts in a bearing is much larger than the flow due to the centrifugal effects discussed previously.

The model is used for the prediction of the layer thickness as a function of time and position across the track in a rolling element bearing. The model takes account of the differences between the individual contacts, due to the difference in the geometry of the outer raceway and inner raceway, and also the variation of the load along the circumference of the bearing. Results of the layer thickness as a function of time are presented for a ball bearing and for a spherical roller bearing. In both cases the effects of the bearing load and rotational speed on the layer thickness decay rate are investigated. It is shown that the decay rate for a ball bearing is significantly larger than for a spherical roller bearing. The effect of the bearing load on the decay rate is small compared to the effect of the rotational speed. However, in all cases the predicted decay periods are small compared to practically observed grease life times. The results show that unless significant replenishment takes place to the track a bearing cannot sustain an adequate lubricant layer. With the developed models it is possible to determine the minimum amount of replenishment needed to maintain a sufficiently film thickness. 


\section{SAMENVATTING}

Om de levensduur van een wentellager te verlengen wordt smeermiddel zoals olie of vet toegepast. Het smeermiddel zorgt ervoor dat de stalen oppervlakken in de contacten tussen de verschillende onderdelen in het lager worden beschermd tegen beschadiging, mits de smeerfilm tussen de oppervlakken dik genoeg is. In de contacten tussen de loopvlakken van de ringen en de rollichamen kunnen contactdrukken van enkele GPa voorkomen. De dikte van de smeerfilm wordt dan onder andere bepaald door de elastische vervorming van de stalen oppervlakken. Dit zijn zogenaamde Elasto-Hydrodynamische gesmeerde (EHL) contacten. Er is sprake van schrale smering wanneer de toevoer van smeermiddel aan de EHL contacten zodanig klein is, dat dit de dikte van de smeermiddelfilm nadelig beïnvloedt. Dit komt vaak voor in vet gesmeerde wentellagers. Aangezien de meeste wentellagers worden gesmeerd met vet, is het nauwkeurig kunnen voorspellen van de dikte van de smeerfilm in schraal gesmeerde wentellagers van groot belang.

Voor schrale smering geldt dat de dikte van de smeerfilm direct gerelateerd is aan de dikte van de lagen smeermiddel die worden toegevoerd aan het EHL contact. In wentellagers wordt de dikte van deze toevoerlagen bepaald door tal van effecten. Voor een betrouwbare voorspelling van de dikte van de smeerfilm in schraal gesmeerde wentellagers zijn modellen nodig die de invloed van deze effecten op de dikte van de toevoerlagen nauwkeurig voorspellen.

In dit proefschrift worden twee effecten onderzocht:

Het eerst effect is de invloed van de centrifugaalkracht op de verandering van de dikte van de toevoerlagen in wentellagers. Als eerste stap is de verandering van de laagdikte op het loopvlak van de binnenring geanalyseerd. Hiervoor zijn de stromingsvergelijkingen afgeleid voor een dun laagje vloeistof op een axisymmetrisch roterend oppervlak. Er is aangetoond dat voor een loopvlak van een wentellager de laagdikte als een functie van de locatie en tijd kan worden beschreven door een quasi-lineaire differentiaal vergelijking. Het model is experimenteel gevalideerd voor bestaande loopvlakken van lagers. De resultaten komen goed overeen met de voorspellingen van het model.

Uitgaande van het model voor een enkel loopvlak is een model ontwikkeld voor de laagdikte verandering ten gevolge van de centrifugaalkracht op alle loopvlakken in het lager, dus op zowel de rollichamen als de ringen. In dit model wordt er van uitgegaan dat het smeermiddel aan de achterkant van elk EHL contact in twee gelijke lagen opsplitst. Om deze aanname te valideren is er een experiment uitgevoerd voor de splitsing van een vloeistoflaag tussen een ring en een plaat.

Als voorbeeld is het model toegepast voor de geometrie van een tonlager en een kegellager. Voor het tonlager blijkt dat, afhankelijk van de vorm van de loopvlakken, twee 
stromingstypen kunnen voorkomen. Desondanks wordt er voor verschillende lagerafmetingen en voor verschillende waarden van de geometrie parameters toch een zelfde afname van de centrale laagdikte voorspeld. Uit het model volgt dat de centrifugale effecten aanzienlijk kunnen bijdragen aan het verminderen van de dikte van de smeermiddellaag binnen de levensduur van het lager.

Het tweede effect dat is onderzocht is de invloed van de stroming in schraal gesmeerde cirkelvormige of elliptische EHL contacten op de verandering van de dikte van de toevoerlagen. De terugstroming of aanvoer van nieuwe vloeistof naar de loopvlakken is hierbij verwaarloosd. Het model dat hiervoor is ontwikkeld is experimenteel gevalideerd met behulp van optische interferometrie. Voor zeer schraal gesmeerde contacten blijkt dat de rolsnelheid geen invloed heeft op de afname van de laagdikte. Ook blijkt dat de afname van de laagdikte kleiner is bij een grotere belasting. Uit het model volgt verder dat de stroming ten gevolge van de contactdruk veel groter is dan de stroming ten gevolge van de centrifugaalkracht.

Het model is toegepast voor het voorspellen van de laagdikte in wentellagers, als een functie van de tijd en positie over de breedte van de loopvlakken. Hierbij wordt rekening gehouden met de verschillen tussen de individuele contacten, ten gevolge van het verschil in de geometrie van het loopvlak van de buitenste en binnenste ring. Ook is de variatie van de belasting op de rollichamen over de omtrek van het lager gemodelleerd. Voor een kogellager en voor een tonlager is met behulp van het model de laagdikte als functie van de tijd bepaald. Voor beide lagers zijn de effecten van de belasting en toerental op de afnamesnelheid van de laagdikte onderzocht. De resultaten laten zien dat de afnamesnelheid voor een kogellager aanzienlijk groter is dan voor een tonlager. Het effect van de lagerbelasting op de afnamesnelheid is klein vergeleken met het effect van de draaisnelheid. Echter, in alle gevallen is de voorspelde periode, waarin een kritische laagdikte wordt bereikt, klein vergeleken met de in de praktijk waargenomen vetlevensduur. Hieruit blijkt dat de toevoer van smeermiddel naar het loopvlak van groot belang is voor het in stand houden van een voldoende dikke smeermiddellaag gedurende langere tijd. Met de ontwikkelde modellen is het mogelijk om de minimale hoeveelheid smeermiddel te bepalen dat moet worden toegevoerd om een voldoende dikke smeermiddellaag te behouden. 


\section{NOMENCLATURE}

\begin{tabular}{|c|c|c|c|}
\hline \multicolumn{4}{|c|}{ Roman scalars } \\
\hline$a$ & $\begin{array}{l}\text { Semi-axes Hertzian contact zone in } x \text { - } \\
\text { direction. }\end{array}$ & $a=\left(3 F R / E^{\prime}\right)^{1 / 3}(2 \kappa \mathcal{E} / \pi)^{1 / 3}$ & {$[\mathrm{~m}]$} \\
\hline$\breve{a}$ & $\begin{array}{l}\text { Semi-axes Hertzian contact zone in } x \text { - } \\
\text { direction for a load } F_{\max }\end{array}$ & & {$[\mathrm{m}]$} \\
\hline$a^{+}, a^{-}$ & $\begin{array}{l}\text { Location of the boundary of the } \\
\text { pressurized region. See Figure } 5.2\end{array}$ & & {$[\mathrm{~m}]$} \\
\hline $\bar{a}^{+}, \bar{a}^{-}$ & $\begin{array}{l}\text { Location of the boundary of the } \\
\text { pressurized region. }\end{array}$ & $\bar{a}^{+}=a^{+} / a, \bar{a}^{-}=a^{-} / a$ & {$[\mathrm{~m}]$} \\
\hline$b$ & $\begin{array}{l}\text { Semi-axes Hertzian contact zone in } y \text { - } \\
\text { direction. }\end{array}$ & $b=a / \kappa$ & {$[\mathrm{m}]$} \\
\hline$\breve{b}$ & $\begin{array}{l}\text { Semi-axes Hertzian contact zone in } y \text { - } \\
\text { direction for a load } F_{\max }\end{array}$ & & {$[\mathrm{m}]$} \\
\hline$B$ & Bearing width & & {$[\mathrm{m}]$} \\
\hline$c$ & Hertzian approach solids & $c=\left(a^{2} /(2 R)\right)(\mathcal{K} / \mathcal{E})$ & {$[\mathrm{m}]$} \\
\hline$\breve{c}$ & $\begin{array}{l}\text { Hertzian approach solids for a load } \\
F_{\max }\end{array}$ & & {$[\mathrm{m}]$} \\
\hline \multirow{2}{*}{ C } & Inverse capillary number & $C=\varepsilon^{2} \sigma_{0} /(\eta U)$ & \\
\hline & Function, see Eqs. (5.19) and (6.23) & & \\
\hline$C_{2}$ & Coefficient, see Eq. (5.29) & & {$\left[\mathrm{m}^{-2} \cdot \mathrm{s}^{-1}\right]$} \\
\hline$C_{3}$ & Coefficient, see Eq. (6.26) & & {$\left[\mathrm{m}^{-2} \cdot \mathrm{s}^{-1}\right]$} \\
\hline$d_{2}$ & Smallest diameter inner raceway SRB & & {$[\mathrm{m}]$} \\
\hline$D_{1}$ & Smallest diameter outer raceway SRB & & {$[\mathrm{m}]$} \\
\hline$D$ & Ratio reduced radii of curvature $R_{x} / R_{y}$ & $D=\kappa^{2}(\mathcal{K}-\mathcal{E}) /\left(\mathcal{E}-\kappa^{2} \mathcal{K}\right)$ & \\
\hline$E^{\prime}$ & Reduced modulus of elasticity & $2 / E^{\prime}=\left(1-v_{1}^{2}\right) / E_{1}+\left(1-v_{2}^{2}\right) / E_{2}$ & {$[\mathrm{~Pa}]$} \\
\hline$E_{1}$ & Elastic modulus surface 1 & & {$[\mathrm{~Pa}]$} \\
\hline$E_{2}$ & Elastic modulus surface 2 & & {$[\mathrm{~Pa}]$} \\
\hline$f r$ & Overrolling frequency & & {$\left[\mathrm{s}^{-1}\right]$} \\
\hline $\begin{array}{l}f_{s}, f_{\theta}, \\
f_{n}\end{array}$ & $\begin{array}{l}\text { Body force components in } s, \theta \text {, and } n \\
\text { direction }\end{array}$ & & {$\left[\mathrm{N} \cdot \mathrm{m}^{-3}\right]$} \\
\hline $\begin{array}{l}\bar{f}_{s}, \bar{f}_{\theta}, \\
\bar{f}_{n}\end{array}$ & $\begin{array}{l}\text { Dimensionless body force components } \\
\text { in } s, \theta \text {, and } n \text { direction }\end{array}$ & $\bar{f}=f H^{2} /(U \eta)$ & {$\left[\mathrm{N} . \mathrm{m}^{-3}\right]$} \\
\hline
\end{tabular}




\begin{tabular}{|c|c|c|c|}
\hline$\hat{f}$ & $\begin{array}{l}\text { Body force, averaged over the } \\
\text { circumference of the bearing }\end{array}$ & & {$\left[\mathrm{N} \cdot \mathrm{m}^{-3}\right]$} \\
\hline$F$ & Load & & {$[\mathrm{N}]$} \\
\hline$F_{i}, F_{o}$ & Load distribution inner/ outer raceway & & {$[\mathrm{N}]$} \\
\hline $\bar{F}_{i}, \bar{F}_{o}$ & Dimensionless load distribution & $\bar{F}_{i}=F_{i} / F_{\max }, \bar{F}_{o}=F_{o} / F_{\max }$ & \\
\hline$F_{c}$ & Centrifugal force & $F_{c}=m_{r} \Omega_{c a}^{2}\left(R_{x, \text { rol }}+R_{x, \text { irw }}\right)$ & {$[\mathrm{N}]$} \\
\hline$\overline{\bar{F}_{c}}$ & Dimensionless centrifugal force & $\bar{F}_{c}=F_{c} / F_{\max }$ & \\
\hline$F_{\max }$ & Maximum static load on inner raceway & $F_{\max }=K_{n}\left(P_{d} \varepsilon /(1-2 \varepsilon)\right)^{n}$ & {$[\mathrm{~N}]$} \\
\hline$F_{r}$ & $\begin{array}{l}\text { Radial bearing load acting on a single } \\
\text { row. }\end{array}$ & & {$[N]$} \\
\hline$h$ & Gap height / film thickness & & {$[\mathrm{m}]$} \\
\hline$h_{c f f}$ & Central fully flooded film thickness & & {$[\mathrm{m}]$} \\
\hline$h_{c s}$ & Central starved film thickness & & [m] \\
\hline$h_{c s, 0}$ & $h_{c s}$ at $t=0$ & & [m] \\
\hline$h_{\text {oil }}$ & $\begin{array}{l}\text { Combined thickness of the layers } \\
\text { supplied to an EHL contact }\end{array}$ & & {$[\mathrm{m}]$} \\
\hline$h_{o i l, n}$ & $\begin{array}{l}\text { Combined thickness of the supply } \\
\text { layers at overrolling } n\end{array}$ & & {$[\mathrm{~m}]$} \\
\hline$h_{\text {oil }, 1}$ & $\begin{array}{l}\text { Fully flooded film thickness at the } \\
\text { outlet of the contact, without reflow }\end{array}$ & & {$[\mathrm{m}]$} \\
\hline$\tilde{h}$ & Free surface layer thickness & & {$[\mathrm{m}]$} \\
\hline $\bar{h}$ & $\begin{array}{l}\text { Dimensionless free surface layer } \\
\text { thickness }\end{array}$ & $\bar{h}=\tilde{h} / H$ & \\
\hline $\bar{h}_{0}$ & Starting position characteristics on $\bar{h}$ & & \\
\hline$\tilde{h}_{\infty}$ & $\begin{array}{l}\text { Free surface layer thickness, averaged } \\
\text { over the length of the track }\end{array}$ & & {$[\mathrm{m}]$} \\
\hline$\tilde{h}_{\infty, 0}$ & $\tilde{h}_{\infty}$ at $t=0$ & & {$[\mathrm{~m}]$} \\
\hline$\tilde{h}_{0}$ & Starting position of characteristics on $\tilde{h}$ & & {$[\mathrm{~m}]$} \\
\hline$\tilde{h}_{0}^{I}$ & $\begin{array}{l}\text { Initial layer thickness distribution as a } \\
\text { function of the position } s=s_{0}\end{array}$ & & {$[\mathrm{~m}]$} \\
\hline$\tilde{h}_{0}^{I I}$ & $\begin{array}{l}\text { Layer thickness as a function of time } \\
t=t_{0} \text { at the inflow } s=0\end{array}$ & & {$[\mathrm{~m}]$} \\
\hline$\tilde{h}_{i r w}$ & $\begin{array}{l}\text { Layer thickness distribution of the layer } \\
\text { at the side of the roller, see Figure } 3.8\end{array}$ & & {$[\mathrm{~m}]$} \\
\hline$\tilde{h}_{c r}$ & Critical layer thickness & & [m] \\
\hline \multirow{3}{*}{$H$} & Dimensionless $h$ & $H=h / c$ & \\
\hline & Characteristic layer thickness & & {$[\mathrm{m}]$} \\
\hline & Initial lubricant layer thickness & & [m] \\
\hline $\mathrm{H}_{2}$ & Initial layer thickness on domain $L_{2}$ & & {$[\mathrm{~m}]$} \\
\hline$H_{\text {oil }}$ & Dimensionless $h_{o i l}$ & $H_{o i l}=h_{o i l} / c$ & \\
\hline
\end{tabular}




\begin{tabular}{|c|c|c|c|}
\hline$H_{c f f}$ & Dimensionless $h_{c f f}$ & $H_{c f f}=h_{c f f} / c$ & \\
\hline$H_{c s}$ & Dimensionless $h_{c s}$ & $H_{c s}=h_{c s} / c$ & \\
\hline$H_{c s, 0}$ & $H_{c s}$ at $\bar{t}=0$ & & \\
\hline$\tilde{H}$ & Dimensionless $\tilde{h}$ & $\tilde{H}=\tilde{h} / c$ & \\
\hline$\tilde{H}_{\infty}$ & Dimensionless $\tilde{h}_{\infty}$ & $\tilde{H}_{\infty}=\tilde{h}_{\infty} / c$ & \\
\hline$\tilde{H}_{\infty, 0}$ & $\tilde{H}_{\infty}$ at $\bar{t}=0$ & & \\
\hline$i$ & Index solids & & \\
\hline$j$ & Index rolling elements & & \\
\hline \multirow{2}{*}{$k$} & Index of the EHL contacts & & \\
\hline & Time step number & & \\
\hline$K_{n}$ & Load deflection factor & $K_{n}=\left(K_{i}^{-1 / n}+K_{o}^{-1 / n}\right)^{-n}$ & {$\left[\mathrm{n} / \mathrm{m}^{\mathrm{n}}\right]$} \\
\hline$K_{i / o}$ & $\begin{array}{l}\text { Load deflection factor for elliptical and } \\
\text { circular contacts }\end{array}$ & $K_{i / o}=\frac{2}{3} E^{\prime} \sqrt{2 R \pi^{2} \mathcal{E} /\left(4 \kappa^{2} \mathcal{K}^{3}\right)}$ & {$\left[\mathrm{n} / \mathrm{m}^{\mathrm{n}}\right]$} \\
\hline$l_{t}$ & Total length of the tracks & & {$[\mathrm{m}]$} \\
\hline$l_{s}, l_{\theta}, l_{n}$ & Scale factors, see Eq. (2.4) & & $\begin{array}{c}{[-],[\mathrm{m}],} \\
{[-]}\end{array}$ \\
\hline \multirow{3}{*}{$L$} & Length in $s$ direction, see Figure 3.4 & & {$[\mathrm{~m}]$} \\
\hline & Characteristic length layer/film & & {$[\mathrm{m}]$} \\
\hline & Dimensionless Moes parameter & $L=\alpha E^{\prime}\left(2 \eta_{0} u_{m} /\left(E^{\prime} R_{x}\right)\right)^{1 / 4}$ & \\
\hline$L_{2}$ & Length in $s$ direction, see Figure 3.4 & & {$[\mathrm{~m}]$} \\
\hline$M$ & Dimensionless Moes parameter & $M=\frac{F}{E^{\prime} R_{x}^{2}}\left(\frac{E^{\prime} R_{x}}{2 \eta_{0} u_{m}}\right)^{3 / 4}$ & \\
\hline$m_{r}$ & Mass rolling element & & [kg] \\
\hline \multirow{3}{*}{$n$} & Number of overrollings & & \\
\hline & Load deflection coefficient & $\begin{array}{l}n=1.11 \text { for line contacts, } \\
n=1.5 \text { for point contacts. }\end{array}$ & \\
\hline & Coordinate normal to the solid surface & & {$[\mathrm{m}]$} \\
\hline $\bar{n}$ & $\begin{array}{l}\text { Dimensionless coordinate normal to the } \\
\text { solid surface }\end{array}$ & $\bar{n}=n / H$ & \\
\hline$n_{c}$ & Number of EHL contacts & & \\
\hline$n_{r}$ & Number of rolling elements per row & & \\
\hline$n_{\text {solids }}$ & $\begin{array}{l}\text { Number of solid objects in rolling } \\
\text { contact per row. (rollers + raceways) }\end{array}$ & & \\
\hline$N$ & Dimensionless Moes parameter & $N=M \sqrt{D}$ & \\
\hline$p$ & Pressure & & {$[\mathrm{Pa}]$} \\
\hline $\bar{p}$ & Dimensionless pressure & $\bar{p}=p H^{2} /(U L \eta)$ & \\
\hline$p_{0}$ & Atmospheric pressure & & {$[\mathrm{Pa}]$} \\
\hline$p_{h}$ & Central Hertzian pressure & $p_{h}=3 F /(2 \pi a b)$ & {$[\mathrm{Pa}]$} \\
\hline
\end{tabular}




\begin{tabular}{|c|c|c|c|}
\hline$\breve{p}_{h}$ & $\begin{array}{l}\text { Central Hertzian pressure for a load } \\
F=F_{\max }\end{array}$ & & {$[\mathrm{Pa}]$} \\
\hline$p_{r}$ & Reference pressure Roelands equation & $p_{r}=1.96 \cdot 10^{8}$ & {$[\mathrm{~Pa}]$} \\
\hline$P$ & Dimensionless pressure distribution & $P=p / p_{h}$ & \\
\hline$P_{H}$ & $\begin{array}{l}\text { Dimensionless Hertzian pressure } \\
\text { distribution }\end{array}$ & & \\
\hline$P_{i}, P_{o}$ & $\begin{array}{l}\text { Dimensionless Hertzian pressure } \\
\text { distribution inner/outer raceway contact }\end{array}$ & $P_{i}=p_{i} / \breve{p}_{h, i}, P_{o}=p_{o} / \breve{p}_{h, i}$ & \\
\hline$P_{d}$ & Diametral clearance & & {$[\mathrm{m}]$} \\
\hline$q$ & Function & & \\
\hline$q_{\text {cage }}$ & $\begin{array}{l}\text { Mass flow rate from cage onto track, } \\
\text { per unit width of the track }\end{array}$ & & {$[\mathrm{kg} / \mathrm{s} / \mathrm{m}$} \\
\hline$q_{s}, q_{\theta}$ & $\begin{array}{l}\text { Mass flow rate in } s / \theta \text { direction per unit } \\
\text { length/width of the track }\end{array}$ & & {$[\mathrm{kg} / \mathrm{s} / \mathrm{m}]$} \\
\hline$q_{y, k}$ & $\begin{array}{l}\text { Mass flow rate in } y \text { direction per unit } \\
\text { length of the track for EHL contact } k\end{array}$ & & {$[\mathrm{~kg} / \mathrm{s} / \mathrm{m}]$} \\
\hline$\hat{q}_{s}, \hat{q}_{y}$ & $\begin{array}{l}\text { Mass flow rate in s/y direction, } \\
\text { integrated over track }\end{array}$ & & {$\left[\mathrm{kg} \cdot \mathrm{s}^{-1}\right]$} \\
\hline$\hat{q}_{y, k}$ & $\begin{array}{l}\text { Mass flow rate in } y \text { direction in EHL } \\
\text { contact } k \text {, integrated over } x\end{array}$ & & {$\left[\mathrm{~kg} . \mathrm{s}^{-1}\right]$} \\
\hline$\hat{q}_{y, i}, \hat{q}_{y, o}$ & $\begin{array}{l}\text { Mass flow rate in } y \text { direction in EHL } \\
\text { contact on the inner/ outer raceway, } \\
\text { integrated over } x\end{array}$ & & {$\left[\mathrm{~kg} \cdot \mathrm{s}^{-1}\right]$} \\
\hline$\hat{Q}_{Y}$ & $\begin{array}{l}\text { Dimensionless mass flow rate in } Y \\
\text { direction, integrated the track }\end{array}$ & $\hat{Q}_{Y} / \hat{q}_{y}=12 \eta_{0} b /\left(h_{\text {oil }}^{3} \rho_{0} p_{h} a n_{c}\right)$ & \\
\hline$\hat{Q}_{Y, k}$ & $\begin{array}{l}\text { Dimensionless mass flow rate in } Y \\
\text { direction in EHL contact } k \text {, integrated } \\
\text { over } X\end{array}$ & $\hat{Q}_{Y, k} / \hat{q}_{y, k}=12 \eta_{0} b /\left(h_{o i l}^{3} \rho_{0} p_{h} a\right)$ & \\
\hline $\begin{array}{l}\hat{Q}_{Y, i} \\
\hat{Q}_{Y, o}\end{array}$ & $\begin{array}{l}\text { Dimensionless mass flow rate in } Y \\
\text { direction in an EHL contact on the } \\
\text { inner/outer raceway, integrated over } X\end{array}$ & $\frac{\hat{Q}_{Y, i}}{\hat{q}_{y, i}}=\frac{\hat{Q}_{Y, o}}{\hat{q}_{y, o}}=\frac{12 \eta_{0} \breve{b}_{i}}{h_{o i l}^{3} \rho_{0} \breve{p}_{h, i} \breve{a}_{i}}$ & \\
\hline$Q_{s}, Q_{\theta}$ & $\begin{array}{l}\text { Volume flow rate in } s / \theta \text { direction per } \\
\text { unit width/length }\end{array}$ & & {$\left[\mathrm{m}^{2} \cdot \mathrm{s}^{-1}\right]$} \\
\hline $\bar{Q}_{s}, \bar{Q}_{\theta}$ & $\begin{array}{l}\text { Dimensionless volume flow rate in } s / \theta \\
\text { direction per unit width/length }\end{array}$ & $\bar{Q}=Q /(H U)$ & \\
\hline$r_{n}$ & Relative film thickness at overrolling $n$ & $r_{n}=h_{o i l, n} / h_{o i l, 1}$ & \\
\hline$r$ & $\begin{array}{l}\text { Radius as function of } s \text {, defining the } \\
\text { shape of an axisymmetric solid surface, } \\
\text { see Figure } 2.2 \text { and Figure } 3.1\end{array}$ & & {$[\mathrm{~m}]$} \\
\hline $\bar{r}$ & Dimensionless radius & $\bar{r}=r / L$ & \\
\hline$R_{x, i}$ & $\begin{array}{l}\text { Radius of curvature surface } i \text { in } x \\
\text { direction }\end{array}$ & & {$[\mathrm{m}]$} \\
\hline$R_{y, i}$ & $\begin{array}{l}\text { Radius of curvature surface } i \text { in } y \\
\text { direction }\end{array}$ & & {$[\mathrm{m}]$} \\
\hline
\end{tabular}




\begin{tabular}{|c|c|c|c|}
\hline$R_{x}$ & $\begin{array}{l}\text { Reduced radius of curvature in } \mathrm{x} \\
\text { direction }\end{array}$ & $1 / R_{x}=1 / R_{x, 1}+1 / R_{x, 2}$ & {$[\mathrm{~m}]$} \\
\hline$R_{y}$ & $\begin{array}{l}\text { Reduced radius of curvature in y } \\
\text { direction }\end{array}$ & $1 / R_{y}=1 / R_{y, 1}+1 / R_{y, 2}$ & {$[\mathrm{~m}]$} \\
\hline$R$ & Reduced radius of curvature & $1 / R=1 / R_{x}+1 / R_{y}$ & {$[\mathrm{~m}]$} \\
\hline$R_{a}$ & Average surface roughness & & \\
\hline$R_{\text {disk }}$ & Track radius on the disk, see Figure 5.9 & & {$[\mathrm{~m}]$} \\
\hline$R_{i r w}$ & Radius inner raceway, see Figure 3.4 & & {$[\mathrm{~m}]$} \\
\hline$R_{\text {rol }}$ & Radius roller, see Figure 3.4 & & {$[\mathrm{~m}]$} \\
\hline $\operatorname{Re}$ & Reynolds’ number & $\operatorname{Re}=\rho U L / \eta$ & \\
\hline$R_{\text {crol }}$ & $\begin{array}{l}\text { Distance between the center of the } \\
\text { roller and the rotational axis of the } \\
\text { bearing }\end{array}$ & & {$[\mathrm{m}]$} \\
\hline$S$ & Coordinate on axisymmetric surface & & [m] \\
\hline$s_{0}$ & Starting position of characteristics on $s$ & & [m] \\
\hline $\bar{s}$ & Dimensionless coordinate & $\bar{s}=s / L$ & \\
\hline$\overline{S_{0}}$ & Starting position characteristics on $\bar{s}$ & & \\
\hline $\mathcal{S}$ & Shape factor & $\mathcal{S}=\left(\mathcal{E}-\kappa^{2} \mathcal{K}\right) /\left(\mathcal{K}-\kappa^{2} \mathcal{K}\right)$ & \\
\hline$t$ & Time & & {$[\mathrm{s}]$} \\
\hline$t_{0}$ & Starting position characteristics on $t$ & & {$[\mathrm{~s}]$} \\
\hline$t_{c r}$ & Critical time & & {$[\mathrm{s}]$} \\
\hline \multirow{2}{*}{$\bar{t}$} & Scaled time, centrifugal effect & $\bar{t}=t U / L=t / \tau_{c}$ & \\
\hline & Scaled time, contact pressure effect & $\bar{t}=t / \tau$ & \\
\hline$\overline{t_{0}}$ & Starting position characteristics on $\bar{t}$ & & {$[\mathrm{~s}]$} \\
\hline$T$ & Temperature & & {$\left[{ }^{\circ} \mathrm{C}\right]$} \\
\hline$u_{m}$ & Average velocity solid surfaces & $u_{m}=\frac{1}{2}\left(u_{1}+u_{2}\right)$ & {$[\mathrm{m} / \mathrm{s}]$} \\
\hline$u_{1}, u_{2}$ & Velocity solid surfaces 1 and 2 & & {$[\mathrm{~m} / \mathrm{s}]$} \\
\hline$U$ & Reference velocity & $U=\rho \Omega^{2} L H^{2} / \eta$ & {$[\mathrm{m} / \mathrm{s}]$} \\
\hline$v_{s}, v_{\theta}$ & $\begin{array}{l}\text { Flow velocity tangential to the solid } \\
\text { surface in } s / \theta \text { direction. }\end{array}$ & & {$[\mathrm{m} / \mathrm{s}]$} \\
\hline $\bar{v}_{s}, \bar{v}_{\theta}$ & Dimensionless $v_{s}$ and $v_{\theta}$ & $\bar{v}_{s}=v_{s} / U, \quad \bar{v}_{\theta}=v_{\theta} / U$ & \\
\hline$w$ & $\begin{array}{l}\text { Flow velocity normal to the solid } \\
\text { surface }\end{array}$ & & {$[\mathrm{m} / \mathrm{s}]$} \\
\hline $\bar{w}$ & Dimensionless $w$ & $\bar{w}=w / W$ & \\
\hline$W$ & $\begin{array}{l}\text { Reference velocity normal to the solid } \\
\text { surface }\end{array}$ & $W=\varepsilon U$ & {$[\mathrm{~m} / \mathrm{s}]$} \\
\hline$x$ & Coordinate & & {$[\mathrm{m}]$} \\
\hline$X$ & Dimensionless coordinate & $X=x / a$ & \\
\hline$y$ & Coordinate & & {$[\mathrm{m}]$} \\
\hline
\end{tabular}




\begin{tabular}{|l|l|l|l|}
\hline$Y$ & Dimensionless coordinate & $Y=y / b$ & \\
\hline$Y_{0}$ & Starting position characteristics on $Y$ & & \\
\hline$z_{r}$ & Viscosity pressure index (Roelands) & $\alpha p_{r} / z_{r}=\log \left(\eta_{0}\right)+9.67$ & \\
\hline$Z$ & $\begin{array}{l}\text { Position on the axis of rotational } \\
\text { symmetry }\end{array}$ & & {$[\mathrm{m}]$} \\
\hline $\bar{Z}$ & Dimensionless $z$ & $\bar{Z}=z / L$ & \\
\hline
\end{tabular}

\section{Greek scalars}

\begin{tabular}{|c|c|c|c|}
\hline$\alpha$ & Viscosity pressure coefficient & & {$[1 / \mathrm{Pa}]$} \\
\hline$\alpha^{\prime}$ & Angle tapered raceway, see Figure 2.4 & & [rad] \\
\hline $\bar{\alpha}$ & $\begin{array}{l}\text { Dimensionless viscosity pressure } \\
\text { coefficient }\end{array}$ & $\bar{\alpha}=\alpha p_{h}$ & \\
\hline$\beta$ & Angle spherical raceway, see Figure 2.6 & & [rad] \\
\hline \multirow[b]{2}{*}{$\gamma$} & Film reduction parameter & & \\
\hline & $\begin{array}{l}\text { Angle between the roller axis } z_{\text {rol }} \text { and } \\
\text { the inner raceway axis } z_{i r w}\end{array}$ & & [rad] \\
\hline \multirow{2}{*}{$\delta$} & Thin layer Reynolds number & $\delta=\varepsilon^{2} \operatorname{Re}$ & \\
\hline & Mutual approach & & [m] \\
\hline$\Delta$ & Dimensionless mutual approach & $\Delta=\delta / c$ & \\
\hline \multirow{2}{*}{$\varepsilon$} & Load distribution factor & & \\
\hline & Ratio $\varepsilon=H / L$ & & \\
\hline $\mathcal{E}$ & Elliptic integral (second kind) & $\mathcal{E}=\int_{0}^{\pi / 2} \sqrt{1-\left(1-\kappa^{2}\right) \sin ^{2}(\psi)} d \psi$ & \\
\hline$\eta$ & Dynamic viscosity & & [Pa.s] \\
\hline$\eta_{\text {dim }}$ & Constant Roelands equation & $\eta_{\mathrm{dim}}=1 \mathrm{~Pa} \cdot \mathrm{s}$ & [Pa.s] \\
\hline$\eta_{0}$ & Ambient dynamic viscosity & & [Pa.s] \\
\hline $\bar{\eta}$ & Dimensionless viscosity & $\bar{\eta}=\eta / \eta_{0}$ & \\
\hline \multirow[t]{2}{*}{$\theta$} & $\begin{array}{l}\text { Angular location on axisymmetric } \\
\text { surface }\end{array}$ & & [rad] \\
\hline & Fractional film content & $\theta=h_{\text {oil }} / h$ & \\
\hline$\phi$ & Integration variable & & \\
\hline$\phi_{a}$ & $\begin{array}{l}\text { Ratio length Hertzian contacts on inner } \\
\text { and outer raceway }\end{array}$ & $\phi_{a}=\breve{a}_{i} / \breve{a}_{o}$ & \\
\hline$\phi_{b}$ & $\begin{array}{l}\text { Ratio width Hertzian contacts on inner } \\
\text { and outer raceway }\end{array}$ & $\phi_{b}=\breve{b}_{i} / \breve{b}_{o}$ & \\
\hline$\psi$ & $\begin{array}{l}\text { Angular location along the } \\
\text { circumference of the bearing }\end{array}$ & & [rad] \\
\hline$\psi_{j}$ & Angular location rolling element $j$ & & [rad] \\
\hline$\psi_{l}$ & Angular location boundary load zones & & [rad] \\
\hline
\end{tabular}




\begin{tabular}{|c|c|c|c|}
\hline$\kappa_{s}, \kappa_{\theta}$ & $\begin{array}{l}\text { Principal normal curvatures } \\
\text { axisymmetric surface }\end{array}$ & & {$\left[\mathrm{m}^{-1}\right]$} \\
\hline \multirow{2}{*}{$\kappa$} & Ellipticity parameter & $R_{x} / R_{y}=\kappa^{2}(\mathcal{K}-\mathcal{E}) /\left(\mathcal{E}-\kappa^{2} \mathcal{K}\right)$ & \\
\hline & Twice the mean curvature & $\kappa=\kappa_{s}+\kappa_{\theta}$ & {$\left[\mathrm{m}^{-1}\right]$} \\
\hline$\kappa_{2}$ & Squared curvature & $\kappa_{2}=\kappa_{s}^{2}+\kappa_{\theta}^{2}$ & {$\left[\mathrm{~m}^{-1}\right]$} \\
\hline $\bar{\kappa}$ & Dimensionless curvature & $\bar{\kappa}=\kappa L$ & \\
\hline$\overline{\kappa_{2}}$ & Dimensionless squared curvature & $\bar{\kappa}_{2}=\bar{\kappa}_{s}^{2}+\bar{\kappa}_{\theta}^{2}$ & \\
\hline$\tilde{\kappa}$ & $\begin{array}{l}\text { Dimensionless curvature at the gas- } \\
\text { liquid interface, see Eq. (2.22) }\end{array}$ & & \\
\hline $\mathcal{K}$ & Elliptic integral (first kind) & $\mathcal{K}=\int_{0}^{\pi / 2}\left(1-\left(1-\kappa^{2}\right) \sin ^{2}(\psi)\right)^{-1 / 2} d \psi$ & \\
\hline$\lambda$ & Coefficient & $\lambda=12 \eta_{0} u_{m} a /\left(c^{2} p_{h}\right)$ & \\
\hline$\lambda_{r r}$ & $\begin{array}{l}\text { Time between exchange position rolling } \\
\text { elements }\end{array}$ & $\lambda_{r r}=2 \pi /\left(n_{r} \Omega_{c a}\right)$ & [s] \\
\hline$v$ & Kinematic viscosity & & {$\left[\mathrm{m}^{2} / \mathrm{s}\right]$} \\
\hline$v_{i}$ & Poisson ration surface $i$ & & \\
\hline$\xi$ & $\begin{array}{l}\text { Parameter characteristic curves, see } \\
\text { Figure } 2.3\end{array}$ & & \\
\hline$\rho$ & Density & & {$\left[\mathrm{kg} / \mathrm{m}^{3}\right]$} \\
\hline$\rho_{0}$ & Ambient density & & {$\left[\mathrm{kg} / \mathrm{m}^{3}\right]$} \\
\hline$\rho_{\kappa}$ & Radius of curvature, see Figure 3.4 & & {$\left[\mathrm{~m}^{-1}\right]$} \\
\hline $\bar{\rho}$ & Dimensionless density & $\bar{\rho}=\rho / \rho_{0}$ & \\
\hline $\bar{\rho}_{c}$ & $\begin{array}{l}\text { Dimensionless density at the center of } \\
\text { the Hertzian contact area. }\end{array}$ & & \\
\hline$\sigma$ & Parameter initial curve, see Figure 2.3 & & \\
\hline$\sigma_{0}$ & Surface tension coefficient & & {$[\mathrm{N} / \mathrm{m}]$} \\
\hline$\tau_{c}$ & Time scale parameter, centrifugal effect & $\tau_{c}=\eta_{0} /\left(\rho_{0} \Omega^{2} H^{2}\right)$ & {$[\mathrm{s}]$} \\
\hline$\tau$ & Time scale parameter, pressure effect & $\tau=\frac{3}{2} \eta_{0} l_{t} b^{2} /\left(p_{h} a c^{2} n_{c}\right)$ & {$[\mathrm{s}]$} \\
\hline$\varphi$ & Variable Hertzian pressure distribution & & \\
\hline$\Omega$ & Angular velocity & & {$[\mathrm{rad} / \mathrm{s}]$} \\
\hline$\Omega_{c a}$ & $\begin{array}{l}\text { Angular velocity of the cage, relative to } \\
\text { the fixed outer raceway (Eq. (3.30)) }\end{array}$ & & {$[\mathrm{rad} / \mathrm{s}]$} \\
\hline$\Omega_{i r w}$ & Angular velocity of the inner raceway & & {$[\mathrm{rad} / \mathrm{s}]$} \\
\hline$\Omega_{\text {rol }}$ & $\begin{array}{l}\text { Angular velocity of the roller, relative } \\
\text { to the cage (Eq. (3.31)) }\end{array}$ & & {$[\mathrm{rad} / \mathrm{s}]$} \\
\hline \multicolumn{4}{|c|}{ Matrices / vectors } \\
\hline$\tau$ & Deviatoric stress & & {$[\mathrm{Pa}]$} \\
\hline $\bar{\tau}$ & Dimensionless $\tau$ & $\overline{\boldsymbol{\tau}}=\boldsymbol{\tau} H /(U \eta)$ & \\
\hline $\mathbf{e}_{\mathbf{z}}$ & Unit vector in z-direction & & \\
\hline
\end{tabular}




\begin{tabular}{|l|l|l|c|}
\hline $\mathbf{e}_{1}, \mathbf{e}_{2}, \mathbf{n}$ & $\begin{array}{l}\text { Unit orthogonal vectors at the solid } \\
\text { surface }\end{array}$ & & \\
\hline $\mathbf{f}$ & Body force vector & {$\left[\mathrm{N} . \mathrm{m}^{-3}\right]$} \\
\hline$\overline{\mathbf{f}}$ & Dimensionless body force vector & $\overline{\mathbf{f}}=\mathbf{f} H^{2} /(\mathrm{U} \eta)$ & \\
\hline $\mathbf{f}_{R}$ & $\begin{array}{l}\text { Body force vector relative to the } \\
\text { rotating frame. }\end{array}$ & & {$\left[\mathrm{N} . \mathrm{m}^{-3}\right]$} \\
\hline $\mathbf{R}$ & Position vector & & {$[\mathrm{m}]$} \\
\hline & Rotation matrix & {$[-]$} \\
\hline $\mathbf{r}$ & Position vector fluid particle & {$[\mathrm{m}]$} \\
\hline$\tilde{\mathbf{t}}$ & $\begin{array}{l}\text { Tangent vector relative to the fluid } \\
\text { surface }\end{array}$ & & \\
\hline $\mathbf{T}$ & Translation vector & & {$[\mathrm{m}]$} \\
\hline $\mathbf{u}$ & Flow velocity vector & {$[\mathrm{m} / \mathrm{s}]$} \\
\hline $\mathbf{x}_{1,2,3, \text { rol }}$ & $\begin{array}{l}\text { Cartesian coordinate systems, see } \\
\text { Figure B.1 }\end{array}$ & {$[\mathrm{m}]$} \\
\hline
\end{tabular}

\section{Super-/ subscripts}

\begin{tabular}{|l|l|}
\hline$\cdot$ & Derivative with respect to $s$ \\
\hline- & Derivative with respect to $t$ \\
\hline- & Scaled variable or function \\
\hline$\sim$ & Free surface \\
\hline$\infty$ & Averaged over the length of the track \\
\hline$c a$ & Cage \\
\hline \multirow{2}{*}{$\boldsymbol{l}$} & Index of the axisymmetric surface on which the fluid layer flows. \\
\cline { 2 - 3 } Node number in s-direction \\
\hline$j$ & Inner raceway \\
\hline$k$ & Index of the rolling elements \\
\cline { 2 - 2 } & Time step \\
\hline$n$ & Normal direction \\
\hline o/ orw & Outer raceway \\
\hline rol & Roller \\
\hline$r w$ & Raceway \\
\hline$S$ & Direction along coordinate $s$ \\
\hline$\theta$ & Circumferential direction \\
\hline Abbreviations \\
\hline EHL & Elasto-Hydrodynamic Lubrication \\
\hline SRB & Spherical Roller Bearing \\
\hline TRB & Tapered Roller Bearing \\
\hline DGBB & Deep Groove Ball Bearing \\
\hline
\end{tabular}

Some symbols have more than one meaning. However, generally at the first use in a chapter or section the appropriate meaning is explicitly stated. 


\section{Chapter 1}

\section{INTRODUCTION}

\subsection{Rolling element bearings}

Bearings are used in mechanical systems to constrain the relative motion between two parts. For example, a wheel of a car is permitted to perform a rotary motion around the wheel axle, but it is constrained to move in any translational direction. For optimal performance it is often required to minimize the friction of a bearing. A successful method to reduce friction is to apply rolling elements between two objects in relative motion. This principle is used in rolling element bearings, resulting in a tremendous reduction of friction, also during the start-up of the motion.

Typically, a rolling element bearing consists of an inner ring, an outer ring, one or two rows of rolling elements and a cage to prevent contact between the rolling elements. Usually, a bearing is lubricated with oil or grease. A bearing can be equipped with a seal, preventing dirt to enter and lubricant to leave the bearing. The high load and high speed capacity combined with a low friction, a long service life, low costs and well developed standardization have lead to the enormous success of rolling element bearings today.

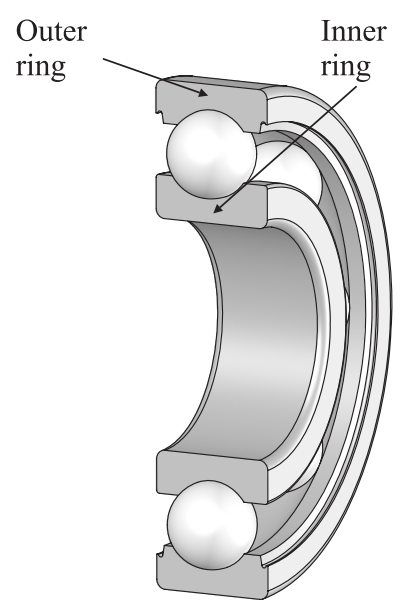

Deep groove ball bearing (DGBB)

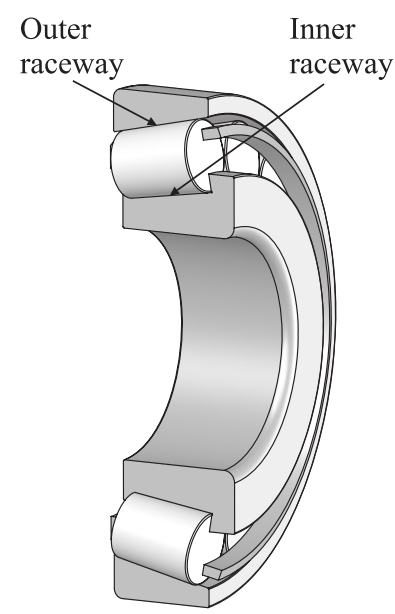

Tapered roller bearing (TRB)

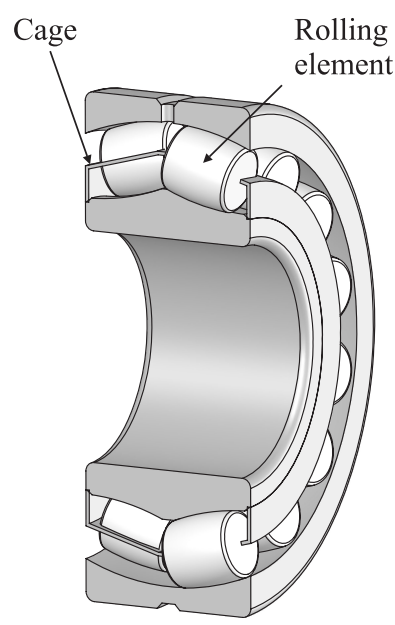

Spherical roller bearing (SRB)

Figure 1.1: Sectional view of three rolling element bearing types. 
Many types of rolling element bearings have been developed. In Figure 1.1 three bearing types are shown, which will be used in the examples given in this thesis. The most widely used rolling element bearing type is the single row deep groove ball bearing. It can carry a radial load and a moderate axial load, i.e. a thrust load. The tapered roller bearing, which was patented in 1898 by Henry Timken, is suited to withstand a relatively high radial and thrust load. In 1917 Sven Wingquist invented the spherical roller bearing with characteristic barrel shaped rollers. An important advantage of this bearing type is that it can endure a relatively large misalignment of the inner and outer ring combined with a high load carrying capacity.

The choice for a certain bearing type depends on the mix of requirements regarding load, speed, precision, stiffness, misalignment allowance, available space, costs, etc, see [2]. An extensive overview of the history of bearings, lubrication and tribology in general, is provided by Dowson [25].

\subsection{Lubrication}

Modern rolling element bearings are capable of operating at high loads for long periods of time. Due to the geometry of the rollers and the raceways, the contact areas can be very small. These small contact areas have to be capable of carrying the load that acts on the bearing. Consequently, commonly contact pressures of several GPa occur. Under these conditions the performance and lifespan of the bearing depends, amongst others, on the ability of the lubricant to form a lubricant film that separates the steel surface of the rolling elements and that of the raceways. This lubricant film prevents the wear of the steel surfaces and may also act as a coolant.

\subsubsection{Elasto - Hydrodynamic Lubrication}

Essential for the build-up of a lubricant film in the contact is the hydrodynamic effect. Due to the converging gap between the rolling elements and the raceway and the motion of the steel surfaces, pressure is generated in the lubricant film. When this pressure is large enough to carry the load that acts normal to the contact, then a full lubricant film is formed. For the high contact pressures that appear in rolling element bearings, the elastic deformation of the rolling element and that of the raceway has a significant effect on the film thickness and shape. This lubrication regime is called Elasto-Hydrodynamic Lubrication (EHL). Moreover, for the very high pressures the viscosity of the lubricant typically increases exponentially with the pressure, which strongly affects the lubricant film formation. In EHL these lubricant films are typically very thin, from tenths of micro-meters to tens of nano-meters. 
The amount of lubricant supplied to an EHL contact can have an influence on the film thickness. This is illustrated in Figure 1.2, which shows the film thickness and pressure distribution for an EHL contact between two rollers operating under fully flooded and under starved lubrication conditions. When the contact operates under fully flooded conditions the gap between solid surfaces is completely filled with lubricant, at least until the point where significant pressure build up starts in the inlet to the contact. When the contact operates under starved conditions the lubricant supply to the contact is insufficient to fill the converging gap between the solid surfaces, resulting in a delay of the pressure build up and a higher pressure gradient at the inlet to the contact. Under these conditions the film thickness can be significantly smaller than under fully flooded conditions.

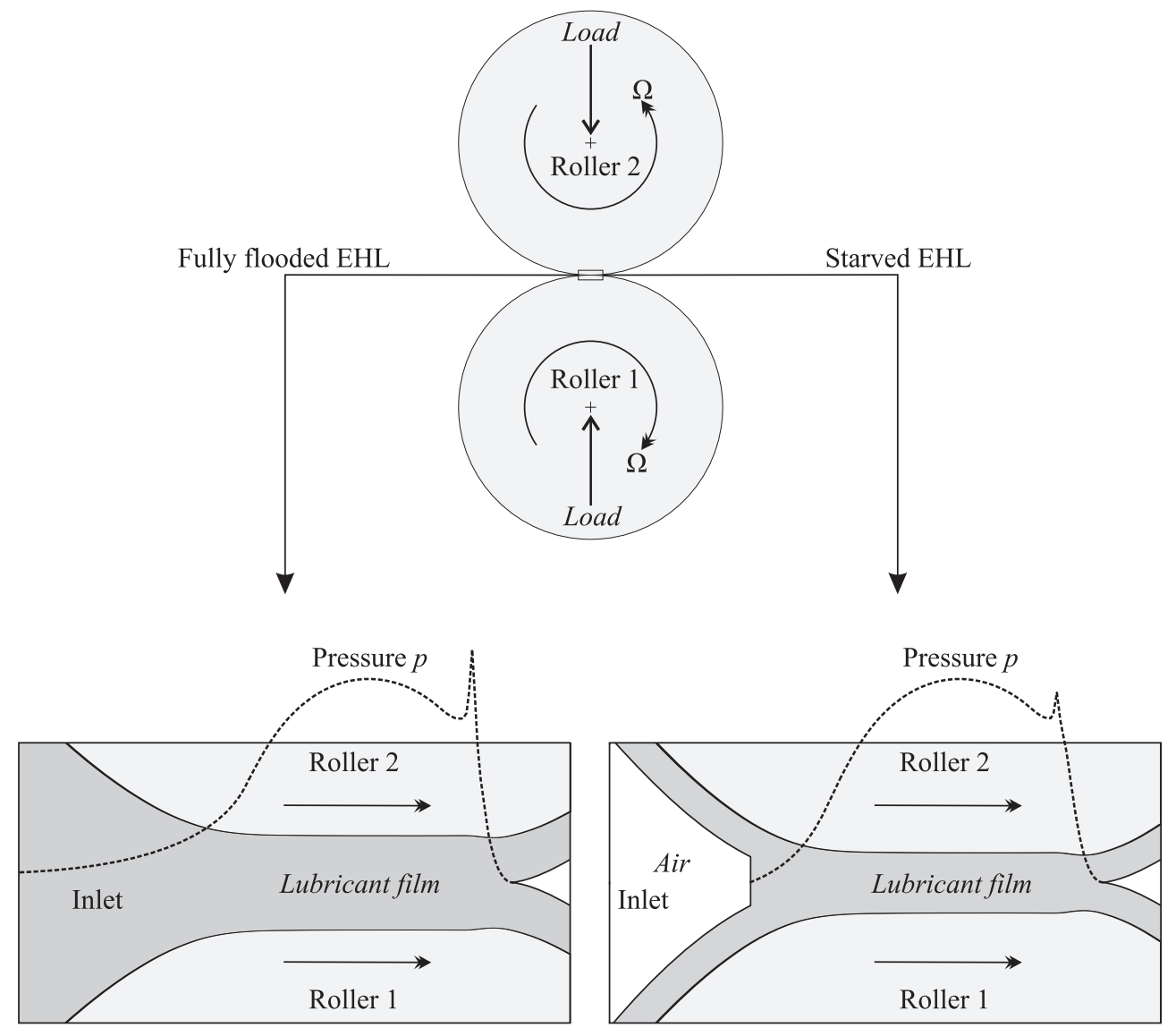

Figure 1.2: Elasto-Hydrodynamically Lubricated contact operating at fully flooded (left) and starved conditions (right). 
Starvation is characteristic for contacts operating at conditions in which the lubricant layers supplied to these contacts are very thin. In rolling element bearings this typically occurs when the track that is formed due to the overrolling of the lubricant layer is still present in the inlet to the next EHL contact. This is illustrated in Figure 1.3, where a close-up is shown of the area around two EHL contacts operating under starved conditions. In the figure, the lubricant layer on the raceway is shown, but the lubricant layer on the rollers is omitted. The dark grey area is the contact region. The layer thickness, the height of the gap between the solid surfaces and the size of the contact area are exaggerated for clarity. At the outlet and the inlet to the contact a track is shown in the middle of two levees of lubricant. Although there is a small reflow effect visible at the outlet of the contact, for this specific case the lubricant is unable to flow onto the track from the sides in between two consecutive overrollings of the track. In that case the thickness of the layers supplied to the next contact is of the same order of magnitude as the film thickness in the previous contact. This is more likely to occur at high speed, high lubricant viscosity and/or a large contact width. Furthermore, it depends on the volume of lubricant available for replenishment, see Cann et al. [9]. Also, grease lubricated contacts are prone to starvation, as will be explained in more detail in the next section.

\subsubsection{Grease lubrication}

Grease is "a solid to semi-fluid product or dispersion of a thickening agent in a liquid lubricant” [1]. Greases that are used to lubricate rolling element bearings consist mainly of oil that is mixed with a smaller portion (5\%-20\%) of thickener. Grease has many advantages and is easy to use. Due to its composition the grease shows a solid-like behavior at low shear rates. Therefore, it does not easily leak out of the bearing. Furthermore, it provides a good corrosion protection and it generally provides low friction because, during the initial phase of bearing operation, most of the initial grease volume is pushed to the side onto the covers/shields/seals, so that churning losses are minimized. Also, the pushed aside grease acts as an additional protection of the roller raceway contacts against contamination. These properties provide cost savings on seals, bearing housing, relubrication systems, maintenance and operation, compared to oil lubricated bearing systems. Consequently, more than $90 \%$ of the rolling bearings are lubricated with grease.

Initially a bearing is filled for approximately $30 \%$ with grease, see Lugt [49]. During the initial overrollings, most of the grease will be pushed to the side of the rolling track. Due to the contact stresses the thickener structure of the remaining grease breaks down, releasing the base oil. In this phase the roller raceway contacts operate in the starved regime. Evidence of starvation was already obtained in 1979 by Wilson [75], who measured the film thickness in rolling element bearings and showed that after the initial churning phase a decrease in film thickness occurs. In the starved regime the film thickness tends to be determined by a film of base oil, possibly on top of a thickener rich layer, with the worked grease near the track and on the cage acting as a lubricant reservoir, i.e. see Cann et al. [13], Kendall et al. [43], Hurley and Cann [39], Booster and Wilcock [6], Wikström and Höglund $[72,73]$. 


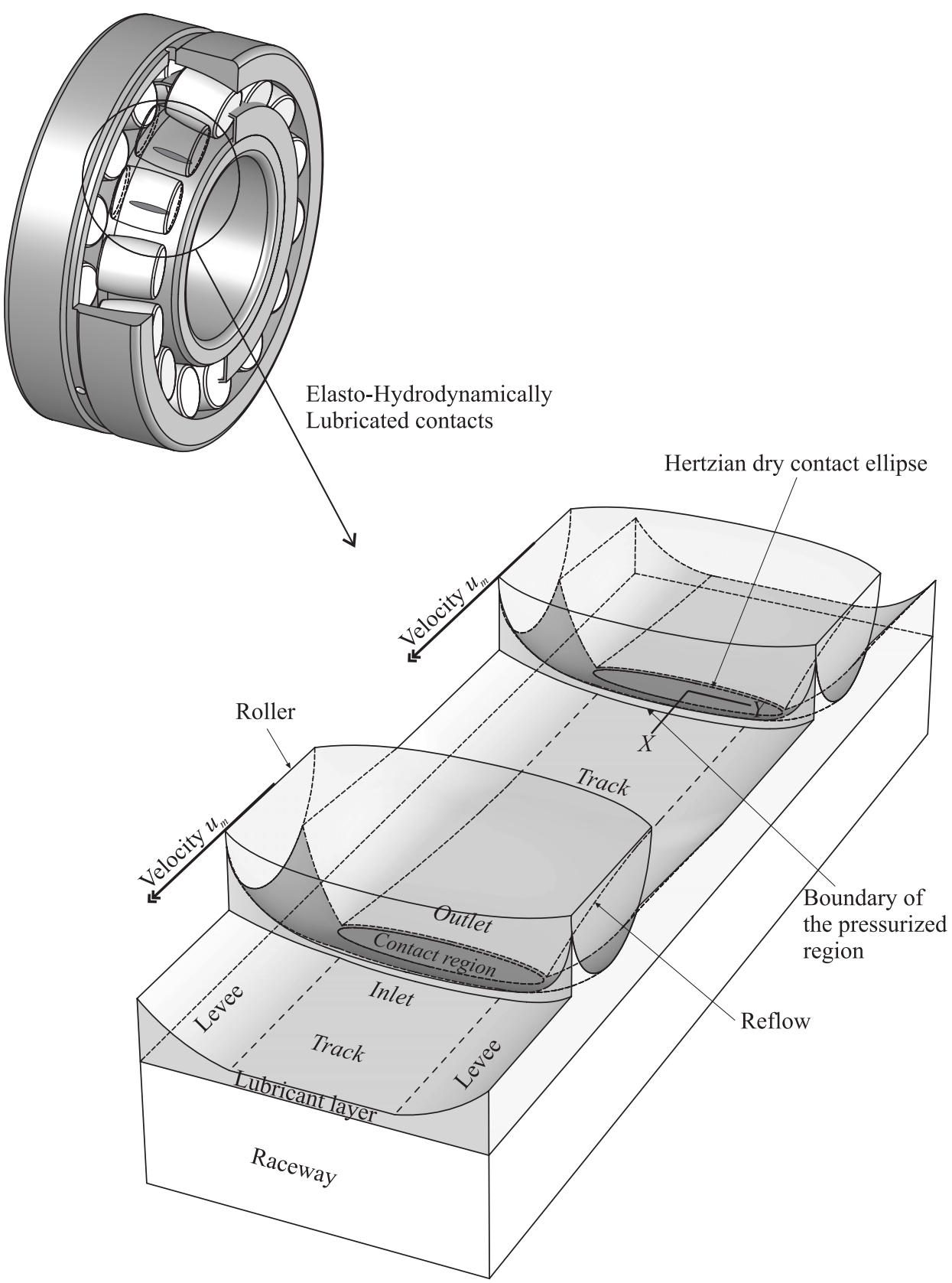

Figure 1.3: In rolling element bearings starvation occurs when the lubricant is unable to replenish the track in between two subsequent overrollings. 
Due to the appearance of starvation grease is often not able to ensure a sufficiently thick lubricant film in the roller-raceway contacts throughout the entire fatigue life of the bearing, and its service life is restricted by what is referred to as grease life. As most bearings nowadays are "greased and sealed" for life, extending this grease life by supplying fresh grease to the bearing is often not an option. Hence, to be able to predict service life of bearings, it is of major importance to be able to predict the grease life, i.e. to predict when the lubricant film formation capability has decreased below a certain critical level.

\subsection{Film thickness prediction}

The prediction of the lifetime and performance of rolling element bearings requires detailed knowledge of the behavior of the EHL contacts for varying conditions of load and lubricant supply during many load cycles. Owing to the development of theoretical models, advanced numerical solution techniques and accurate measurement methods, much is known today about the behavior of EHL contacts, see Dowson [26] and Lee-Prudhoe et al. [47].

The film formation in grease lubricated contacts is affected by many different aspects and further complicated by the complex rheological behavior of grease. However, in the regime after the initial overrollings, grease lubricated contacts can be modelled quite well as oil lubricated starved contacts. Chevalier et al. [18] and Damiens et al. [19] analysed the film thickness in starved circular and elliptical EHL contacts by means of numerical calculations and experiments. They showed that the lubricant film thickness in the contacts is directly related to the thickness and shape of the lubricant layers supplied to the contacts.

However, the prediction of the film thickness in grease lubricated rolling elements bearings is still a major challenge, as the thickness and shape of the layers supplied to the EHL contacts in a bearing application are generally unknown. Many effects are involved that can cause the lubricant to migrate, e.g.:

- Contact forces between de rolling elements and the raceway

- Contact forces between de rolling elements and the cage, see Damiens et al. [21]

- Centrifugal forces, see Gershuni et al. [32] and van Zoelen, et al. [77]

- Gravity: Horizontal or vertical shaft arrangements

- Surface tension, see Åstrom et al. [3] and Gershuni et al. [32]

- Capillary forces, see Jacod [40]

- Ball spin

- Air flow

- Shocks/vibrations

- Transient loading, see Cann and Lubrecht [11]

- $\quad$ Start-stop operation 
Furthermore, the amount of base oil available for lubrication may differ. This is determined by:

- The initial filling and distribution of lubricant, see Lugt et al. [50]

- Grease bleeding: from the cage and the side reservoirs

- $\quad$ Grease degradation, see Cann, et al. [15]

- Evaporation

- Oxidation

Different physical mechanisms may dominate depending on the bearing type, lubricant properties, and the operating conditions, see Cann and Lubrecht [10, 12]. Moreover, the above mentioned effects are not independent. The thickness of the supply layers and thus the film thickness in the EHL contacts is determined by a combination of the effects that expel lubricant from the rolling track and effects that replenish the rolling track (Wikström and Jacobson [74]). This balance is not constant in time and can show chaotic behavior, see Lugt et al. [50].

In order to predict the film thickness in starved lubricated rolling element bearings, new models for the prediction of the supply layer thickness distribution in rolling element bearings are required. 


\subsection{Objectives}

The aim of the research presented in this thesis is to develop a model that predicts the thickness of the lubricant layers supplied to the EHL contacts in rolling element bearings operating under starved conditions.

\section{Two effects are considered (see Figure 1.4):}

- The centrifugal effect; depending on the geometry of the lubricated surfaces, the inertia forces that act on the lubricant layers on the rollers and the raceways can have an effect on the thickness distribution of these layers.

- The contact pressure effect; due to the pressure distribution in the EHL contacts lubricant flows to the sides of the running track, resulting in a decrease of the layer thickness supplied to the EHL contacts.

The influence of relevant parameters on the time variation of the layer thickness distribution is investigated in detail.

\section{Contact pressure effect}

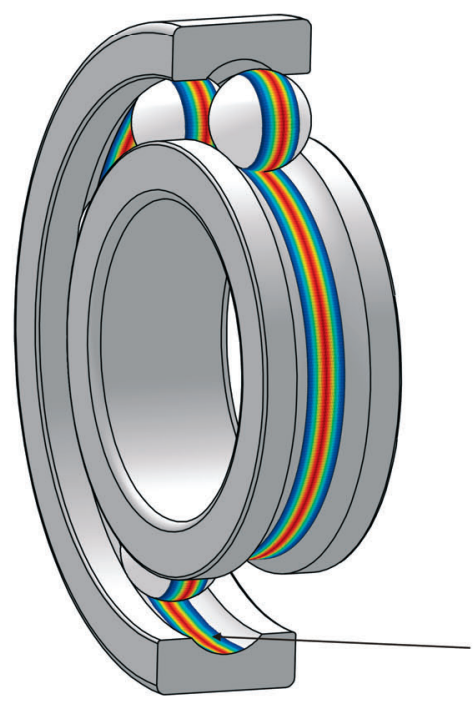

\section{Centrifugal effect}

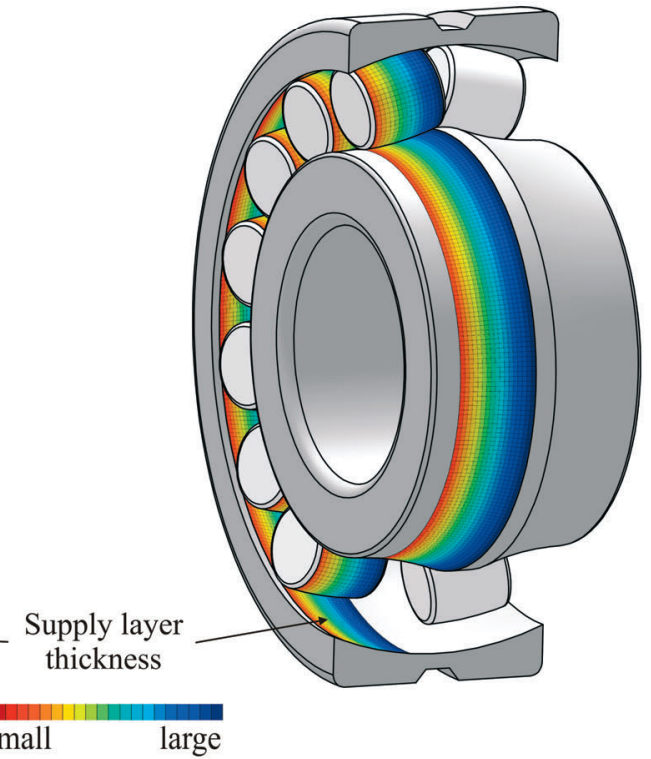

Figure 1.4: Supply layer thickness, illustrating the effect of contact pressure and the centrifugal force on the lubricant layer thickness distribution. 


\subsection{Outline}

First, the centrifugal effects are considered. In Chapter 2 the effect of the centrifugal force on a liquid layer on a raceway is analyzed in relation to the geometry of the raceway.

Based on the single raceway model a model is developed for an entire bearing. It can be used to predict the long term decrease of the supply layer thickness due to centrifugal forces, as a function of the position across the running track, for different types of bearings. This model is presented in Chapter 3.

In addition to the effect of the centrifugal force, the thickness of the lubricant layers on the track is affected by the flow in the EHL contacts. In the second part of this thesis this effect is studied in detail. This requires solving the EHL contact problem. Therefore, in Chapter 4 the standard theoretical model and experimental method to analyze single EHL contact behavior are described.

In Chapter 5 the long term effects of the EHL contact pressure on the layer thickness under starved conditions are considered. A model is presented for the prediction of the change of the layer thickness, assuming that the reflow is negligible. This model is applicable for a single contact situation at a constant load, or a multiple contact situation, where the contact geometry and the load are constant and equal for each contact. This occurs for example in purely axially loaded thrust bearings.

In many cases a bearing is (also) loaded in radial direction. In that case the load varies over the circumference of the bearing. Furthermore, the geometry of the steel surfaces can be different for the inner and outer raceway. In Chapter 6 the model presented in Chapter 5 is extended to include the effects of the load variation and the difference in the geometry of the outer and inner raceway.

Finally, in Chapter 7 the conclusion of the present study and recommendations for future research are given. 


\section{Chapter 2}

\section{CENTRIFUGAL EFFECTS ON FLOW}

In this chapter the subject of investigation is the effect of the centrifugal forces on the flow of an oil layer situated on a raceway. The main part of this research has been published in van Zoelen et al. [77]. Starting from the Navier-Stokes equations, the flow equations for a fluid layer on rotating axisymmetric solid surfaces are derived. For rolling element bearings the model can be simplified to a quasi-linear partial differential equation for the layer thickness, as a function of location and time. This equation can be solved using the method of characteristics. Experiments have been carried out, measuring the changes in time of the thickness of an oil layer on rotating raceways, as a function of the rotational speed and the raceway geometry. The results of the experiments are compared with the predictions of the simplified model. Finally, the effects on the solution of some of the assumptions made to obtain the simplified model are illustrated by means of numerical simulations.

\subsection{Thin layer flow modeling}

In this thesis a layer refers to a thin liquid layer on a solid surface with an air/liquid interface, and a thin liquid layer separating two solids is called a film. (See Figure 2.1). The height of the layer and the film is denoted by $\tilde{h}$ and $h$, respectively. The flow of a liquid layer or a liquid film is characterized by the dimensions of the fluid domain which, in the direction perpendicular to the surface is much smaller than in the direction along the surface. Let $H$ be a characteristic layer/film height and $L$ a characteristic dimension in the other directions, then the ratio $\varepsilon=H / L$ is small. Assuming $\varepsilon^{2} \ll 1$, the flow can be modeled using the lubrication approximation. This greatly simplifies the flow equations.

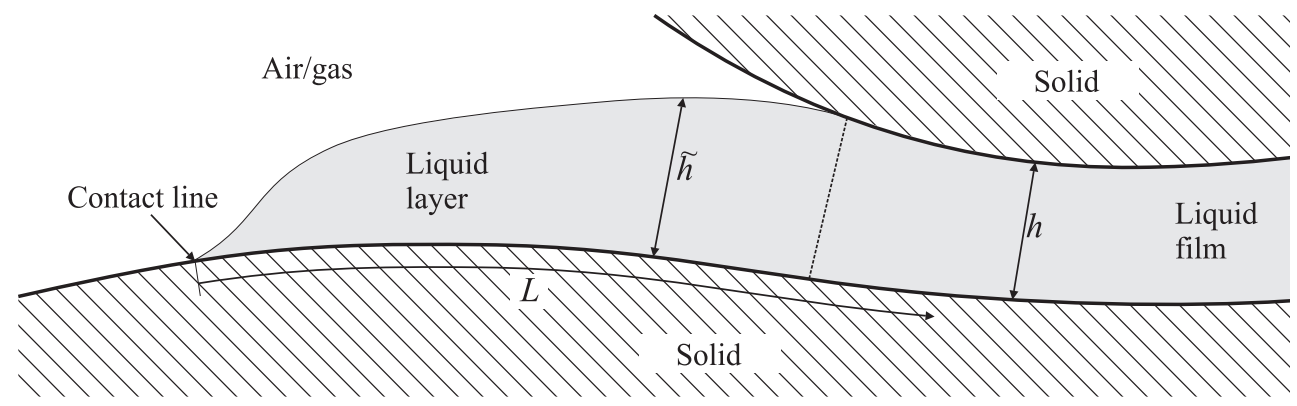

Figure 2.1: Cross section thin liquid film/layer 
Problems that can be characterized as thin layer flow on a solid surface appear in many practical applications, e.g. motion of rain down a window, ice accretion on aircraft wings, flow of mucus in lungs, coating flows and paint films. The simplest form concerns the flow of a homogeneous Newtonian fluid, but also non-Newtonian behavior can occur, in which the viscosity varies with shear stress, temperature and/or pressure. In practical applications there are many other effects that can be important e.g. air flow above the layer, gravity, van der Waals forces, centrifugal forces, surface tension, the Marangoni effect, evaporation, condensation and temperature effects, see O'Brien et al. [55]. For partially wetted systems the dynamics of the contact line, shown in Figure 2.1, can be important, see Diez et al. [24].

The flow of a thin Newtonian fluid layer on a curved two-dimensional surface is treated by Schwartz et al. [61], and the equations for thin layer flow on an arbitrary three-dimensional surface have been presented by Myers et al. [54] and Roy et al. [64]. In the next section Myers' approach is followed to derive the governing equations for the free surface thin layer flow on an object with a smoothly curved axisymmetric surface. Next, this model is used to study the flow on bearing raceways.

\subsection{Theoretical formulation}

\subsubsection{Coordinate system}

In Figure 2.2 an illustration is shown of the configuration considered and the coordinate system that will be used in the description. The solid surface, on which the layer flows is assumed to be non-porous, rigid, and axisymmetric with respect to the $z$ axis. It is parameterized by the variables $s$, which is the arc length of the surface in axial direction, and $\theta$ in circumferential direction. The location of a specific point of the solid surface is given by:

$$
\mathbf{R}(s, \theta)=\left[\begin{array}{c}
x \\
y \\
z
\end{array}\right]=\left[\begin{array}{c}
r(s) \cos (\theta) \\
r(s) \sin (\theta) \\
z(s)
\end{array}\right],
$$

where $r(s)$ is the local radius and $z(s)$ the position on the z-axis as a function of $s$ (See Figure 2.2). The derivatives are $r^{\prime}(s)=d r / d s$ and $z^{\prime}(s)=d z / d s$. The unit orthogonal vectors along the coordinate directions $\left(\mathbf{e}_{1}, \mathbf{e}_{2}, \mathbf{n}\right)$ are defined by:

$$
\begin{aligned}
& \mathbf{e}_{1}=\frac{\partial \mathbf{R}}{\partial s} /\left|\frac{\partial \mathbf{R}}{\partial s}\right|=\left[\begin{array}{c}
r^{\prime}(s) \cos (\theta) \\
r^{\prime}(s) \sin (\theta) \\
z^{\prime}(s)
\end{array}\right], \quad \mathbf{e}_{2}=\frac{\partial \mathbf{R}}{\partial \theta} /\left|\frac{\partial \mathbf{R}}{\partial \theta}\right|=\left[\begin{array}{c}
-\sin (\theta) \\
\cos (\theta) \\
0
\end{array}\right], \\
& \mathbf{n}=-\mathbf{e}_{1} \times \mathbf{e}_{2}=\left[\begin{array}{c}
z^{\prime}(s) \cos (\theta) \\
z^{\prime}(s) \sin (\theta) \\
-r^{\prime}(s)
\end{array}\right], \quad \text { with } \quad z^{\prime}(s)=\sqrt{1-r^{\prime}(s)^{2}}
\end{aligned}
$$




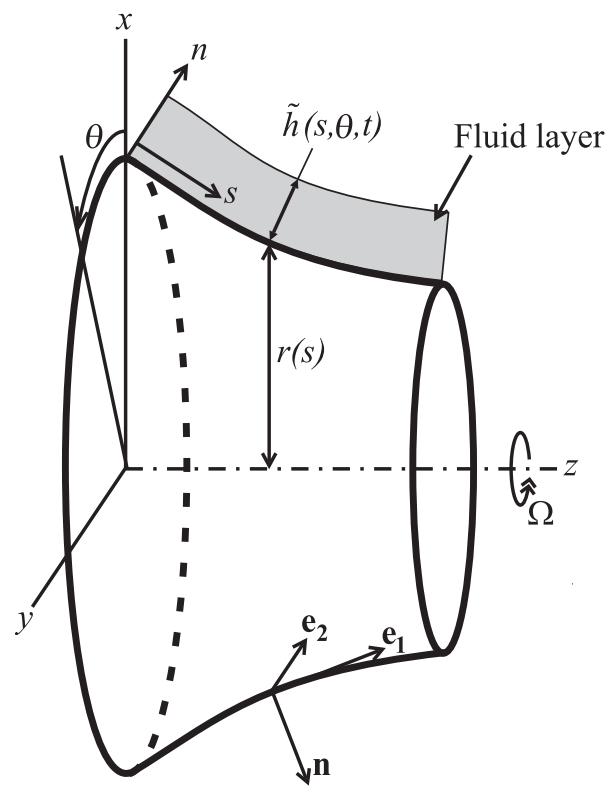

Figure 2.2: Typical configuration of a thin layer with free surface on a surface of revolution and a suitable coordinate system for its description.

The surface rotates at a constant angular velocity $\Omega$. The flow of the layer on the surface is described using a local curvilinear orthogonal coordinate system $(s, \theta, n)$, where $n$ is the coordinate in the normal direction from the surface to a position in the layer. The position vector of a liquid particle in the layer is written as:

$$
\mathbf{r}(s, \theta)=\mathbf{R}(s, \theta)+n \mathbf{n}(s, \theta)
$$

The following scale factors are introduced:

$$
l_{s}(s, n)=\left|\frac{\partial \mathbf{r}}{\partial s}\right|=1-\kappa_{s} n, \quad l_{\theta}(s, n)=\left|\frac{\partial \mathbf{r}}{\partial \theta}\right|=r\left(1-\kappa_{\theta} n\right), \quad l_{n}=\left|\frac{\partial \mathbf{r}}{\partial n}\right|=1,
$$

where $\kappa_{s}$ and $\kappa_{\theta}$ represent the principal normal curvatures in the $s$ and $\theta$ direction, respectively, which are defined according to:

$$
\kappa_{s}(s)=\frac{r^{\prime \prime}(s)}{z^{\prime}(s)}, \quad \kappa_{\theta}(s)=\frac{-z^{\prime}(s)}{r(s)} .
$$

The thickness of the layer on the surface at a given position $(s, \theta)$ and time $t$ will be denoted by $\tilde{h}(s, \theta, t)$. 


\subsubsection{Equations of motion}

The liquid is assumed to behave as a Newtonian fluid, to be incompressible and free of solvents. The dynamic viscosity $\eta$ and the density $\rho$ are assumed to be constant. The Navier-Stokes equations for this case are:

$$
\rho\left(\frac{\partial \mathbf{u}}{\partial t}+(\mathbf{u} \cdot \nabla) \mathbf{u}\right)=\mathbf{f}-\nabla p+\eta \nabla^{2} \mathbf{u},
$$

where $p$ is the pressure, $\mathbf{f}$ is the body force vector and $\mathbf{u}$ is the flow velocity vector. The pressure distribution, the flow velocities, and the body forces are defined relative to a reference frame, which rotates along with the solid surface at a constant angular velocity $\Omega$, where the axis of rotation coincides with the $z$-axis. The body force vector $\mathbf{f}$ is then defined as:

$$
\mathbf{f}=\mathbf{f}_{R}-\rho \Omega^{2} \mathbf{e}_{z} \times\left(\mathbf{e}_{z} \times \mathbf{r}\right)-2 \rho \Omega \mathbf{e}_{z} \times \mathbf{u},
$$

where the second and the third term of the right hand side represent the centrifugal and Coriolis forces, respectively. $\mathbf{f}_{R}$ is a body force vector relative to the rotating reference frame and $\mathbf{e}_{z}=(0,0,1)$ is the unit vector in $z$-direction.

The Navier-Stokes equations written in orthogonal curvilinear coordinates are:

$$
\begin{aligned}
& \rho\left\{\frac{\partial}{\partial t}+\frac{v_{s}}{l_{s}} \frac{\partial}{\partial s}+\frac{v_{\theta}}{l_{\theta}} \frac{\partial}{\partial \theta}+\frac{w}{l_{n}} \frac{\partial}{\partial n}\right\}\left(v_{s} \mathbf{e}_{1}+v_{\theta} \mathbf{e}_{2}+w \mathbf{n}\right)= \\
& \left(f_{s}-\frac{1}{l_{s}} \frac{\partial p}{\partial s}\right) \mathbf{e}_{1}+\left(f_{\theta}-\frac{1}{l_{\theta}} \frac{\partial p}{\partial \theta}\right) \mathbf{e}_{2}+\left(f_{n}-\frac{1}{l_{n}} \frac{\partial p}{\partial n}\right) \mathbf{n}+ \\
& \frac{\eta}{l_{s} l_{\theta} l_{n}}\left\{\frac{\partial}{\partial s}\left(\frac{l_{\theta} l_{n}}{l_{s}} \frac{\partial}{\partial s}\right)+\frac{\partial}{\partial \theta}\left(\frac{l_{s} l_{n}}{l_{\theta}} \frac{\partial}{\partial \theta}\right)+\frac{\partial}{\partial n}\left(\frac{l_{s} l_{\theta}}{l_{n}} \frac{\partial}{\partial n}\right)\right\}\left(v_{s} \mathbf{e}_{1}+v_{\theta} \mathbf{e}_{2}+w \mathbf{n}\right),
\end{aligned}
$$

where $v_{s}=v_{s}(s, \theta, n, t)$ and $v_{\theta}=v_{\theta}(s, \theta, n, t)$ denote the velocity components tangential to the surface and $w=w(s, \theta, n, t)$ represents the velocity in normal direction. The equation of continuity $\nabla \cdot \mathbf{u}=0$ for an incompressible lubricant in curvilinear coordinates is given by:

$$
\frac{\partial}{\partial s}\left(l_{\theta} l_{n} v_{s}\right)+\frac{\partial}{\partial \theta}\left(l_{s} l_{n} v_{\theta}\right)+\frac{\partial}{\partial n}\left(l_{s} l_{\theta} w\right)=0
$$

In case the vector $\mathbf{f}_{R}$ is zero, the body force components Eq. (2.7) in curvilinear coordinate directions are:

$$
\begin{aligned}
& f_{s}(s, \theta, n, t)=\mathbf{f} \cdot \mathbf{e}_{1}=\rho \Omega^{2} r^{\prime}\left(r+n z^{\prime}\right)+2 \rho \Omega r^{\prime} v_{\theta}, \\
& f_{\theta}(s, \theta, n, t)=\mathbf{f} \cdot \mathbf{e}_{2}=-2 \rho \Omega\left(r^{\prime} v_{s}+z^{\prime} w\right), \\
& f_{n}(s, \theta, n, t)=\mathbf{f} \cdot \mathbf{n}=\rho \Omega^{2} z^{\prime}\left(r+n z^{\prime}\right)+2 \rho \Omega z^{\prime} v_{\theta} .
\end{aligned}
$$




\subsubsection{Scaling}

The equations are made dimensionless introducing a characteristic time scale $\tau_{c}$, a characteristic length scale $L$, a characteristic thickness of the liquid layer $H$ and characteristic velocities $U$ and $W$, in the direction along the surface and in the cross layer direction, respectively. The variables used in the following derivation are expressed in dimensionless variables, denoted by overlined symbols:

$$
\begin{array}{lll}
n=H \bar{n}, & \tilde{h}=H \bar{h}, & \kappa=\bar{\kappa} / L, \\
r=L \bar{r}, & s=L \bar{s}, & z=L \bar{z}, \\
v_{s}=U \bar{v}_{s}, & v_{\theta}=U \bar{v}_{\theta}, & w=W \bar{w}, \\
p=\frac{U \eta L}{H^{2}} \bar{p}, & Q=U H \bar{Q}, & \boldsymbol{\tau}=\frac{U \eta}{H} \bar{\tau}, \\
\mathbf{f}=\frac{U \eta}{H^{2}} \overline{\mathbf{f}}, & t=\tau_{c} \bar{t}=\frac{L}{U} \bar{t}, &
\end{array}
$$

where $\kappa$ is a curvature, $Q$ is the rate of volume flow per unit width of the layer, and $\tau$ is the deviatoric stress tensor across the free surface of the layer. Characteristic for thin layer flow problems is that the aspect ratio $\varepsilon^{2}=(H / L)^{2} \ll 1$ and $\delta=\varepsilon^{2} \operatorname{Re} \ll 1$, where $\mathrm{Re}=\rho U L / \eta$ is the Reynolds number. In addition, it is assumed that the typical radius of curvature of the solid surface is large compared to the layer thickness, hence $\varepsilon \bar{\kappa} \ll 1$. The scale factors (2.4) expressed in the non-dimensional variables are:

$$
l_{s}=\left(1-\varepsilon \bar{\kappa}_{s} \bar{n}\right), \quad l_{\theta}=L \bar{r}\left(1-\varepsilon \bar{\kappa}_{\theta} \bar{n}\right), \quad l_{n}=1 .
$$

Substitution of the non-dimensional variables and scaling factors in the continuity relation Eq. (2.9) gives, after neglecting all $\varepsilon \bar{\kappa}$-terms and division by $U$ :

$$
\frac{\partial}{\partial \bar{s}}\left(\bar{r} \bar{v}_{s}\right)+\frac{\partial \bar{v}_{\theta}}{\partial \theta}+\frac{W}{\varepsilon U} \bar{r} \frac{\partial \bar{w}}{\partial \bar{n}}=0
$$

From Eq. (2.13) it follows that $W=\mathrm{O}(\varepsilon U)$, i.e. the velocity component in the $n$-direction must be much smaller than the components along the surface. Substitution of the nondimensional variables and scale factors into the Navier-Stokes equation (2.8), using $W=\varepsilon U$ gives, after multiplication with $H^{2} /(U \mu)$ and neglecting all $\mathrm{O}(\varepsilon \bar{\kappa})$-terms:

$$
\begin{aligned}
& \delta\left\{\frac{\partial}{\partial \bar{t}}+\bar{v}_{s} \frac{\partial}{\partial \bar{s}}+\bar{v}_{\theta} \frac{1}{\bar{r}} \frac{\partial}{\partial \theta}+\bar{w} \frac{\partial}{\partial \bar{n}}\right\}\left(\bar{v}_{s} \mathbf{e}_{1}+\bar{v}_{\theta} \mathbf{e}_{2}+\varepsilon \bar{w} \mathbf{n}\right)= \\
& \left(\overline{f_{s}}-\frac{\partial \bar{p}}{\partial \bar{s}}\right) \mathbf{e}_{1}+\left(\bar{f}_{\theta}-\frac{1}{\bar{r}} \frac{\partial \bar{p}}{\partial \theta}\right) \mathbf{e}_{2}+\left(\bar{f}_{n}-\frac{1}{\varepsilon} \frac{\partial \bar{p}}{\partial \bar{n}}\right) \mathbf{n}+ \\
& \left\{\varepsilon^{2} \frac{1}{\bar{r}} \frac{\partial}{\partial \bar{s}}\left(\bar{r} \frac{\partial}{\partial \bar{s}}\right)+\varepsilon^{2} \frac{1}{\bar{r}^{2}} \frac{\partial^{2}}{\partial \theta^{2}}+\frac{\partial^{2}}{\partial \bar{n}^{2}}\right\}\left(\bar{v}_{s} \mathbf{e}_{1}+\bar{v}_{\theta} \mathbf{e}_{2}+\varepsilon \bar{w} \mathbf{n}\right) .
\end{aligned}
$$


Ordering the terms according to their order of magnitude gives:

$$
\begin{gathered}
\frac{\partial^{2} \bar{v}_{s}}{\partial \bar{n}^{2}}=\frac{\partial \bar{p}}{\partial \bar{s}}-\bar{f}_{s}+\mathrm{O}\left(\varepsilon \bar{\kappa}, \varepsilon^{2}, \delta\right), \\
\frac{\partial^{2} \bar{v}_{\theta}}{\partial \bar{n}^{2}}=\frac{1}{\bar{r}} \frac{\partial \bar{p}}{\partial \theta}-\bar{f}_{\theta}+\mathrm{O}\left(\varepsilon \bar{\kappa}, \varepsilon^{2}, \delta\right),
\end{gathered}
$$

and:

$$
\frac{\partial \bar{p}}{\partial \bar{n}}=\varepsilon \bar{f}_{n}+\mathrm{O}\left(\varepsilon \bar{\kappa}, \varepsilon^{2}, \varepsilon^{2} \delta\right)
$$

To obtain the pressure and the velocities boundary conditions are required. These are discussed in the following section.

\subsubsection{Boundary conditions}

The boundary conditions at the solid/liquid interface and the liquid/air interface are:

1. No-slip condition at the solid surface:

$$
\bar{v}_{s}=\bar{v}_{\theta}=\bar{w}=0 \quad \text { on } \quad \bar{n}=0 .
$$

2. All liquid particles at the liquid/air interface follow the interface. This is defined by the kinematic boundary condition:

$$
\bar{w}=\frac{\partial \bar{h}}{\partial \bar{t}}+\frac{\bar{v}_{s}}{l_{s}} \frac{\partial \bar{h}}{\partial \bar{s}}+\frac{\bar{v}_{\theta} L}{l_{\theta}} \frac{\partial \bar{h}}{\partial \theta} \quad \text { on } \quad \bar{n}=\bar{h} .
$$

3. The liquid/air interface is free of contamination. The shear stresses due to the induced air flow at the interface are assumed to be small, and will not be included in the model, i.e. the shear stress at the interface is thereby assumed to be zero:

$$
\overline{\boldsymbol{\tau}} \cdot \tilde{\mathbf{t}}=0 \Rightarrow \frac{\partial \bar{v}_{s}}{\partial \bar{n}}+\mathrm{O}\left(\varepsilon \kappa, \varepsilon^{2}\right)=\frac{\partial \bar{v}_{\theta}}{\partial \bar{n}}+\mathrm{O}\left(\varepsilon \kappa, \varepsilon^{2}\right)=0 \quad \text { on } \quad \bar{n}=\bar{h}
$$

where $\tilde{\mathbf{t}}$ is the tangent vector relative to the liquid/air surface and $\overline{\boldsymbol{\tau}}$ is the dimensionless deviatoric stress across the free surface.

4. Due to surface tension a jump in the normal stress at the liquid/air interface occurs, which is proportional with the mean curvature of the interface. If $\sigma_{0}$ is the surface tension coefficient, then the dimensionless pressure at the interface is defined according to:

$$
\bar{p}=\bar{p}_{0}-C \tilde{\kappa}+\mathrm{O}\left(\varepsilon^{2}\right) \quad \text { on } \quad \bar{n}=\bar{h},
$$


where $C=\varepsilon^{2} \sigma_{0} / \eta U$ is an inverse capillary number, $\bar{p}_{0}$ is the dimensionless atmospheric pressure and $\tilde{\kappa}$ is the dimensionless curvature of the air/liquid interface:

$$
\tilde{\kappa}=\bar{\kappa}+\varepsilon \bar{\kappa}_{2} \bar{h}+\varepsilon \nabla^{2} \bar{h}+\mathrm{O}\left(\varepsilon^{2}\right) .
$$

Here $\bar{\kappa}=\bar{\kappa}_{s}+\bar{\kappa}_{\theta}$ is twice the dimensionless mean curvature of the solid surface and $\bar{\kappa}_{2}=\bar{\kappa}_{s}^{2}+\bar{\kappa}_{\theta}^{2}$. Neglecting the $\varepsilon \kappa$ terms, the Laplacian of the height of the liquid/air interface is defined by:

$$
\nabla^{2} \bar{h}=\frac{1}{\bar{r}} \frac{\partial}{\partial \bar{s}}\left(\bar{r} \frac{\partial \bar{h}}{\partial \bar{s}}\right)+\frac{1}{\bar{r}^{2}} \frac{\partial^{2} \bar{h}}{\partial \theta^{2}} .
$$

For a detailed derivation of these boundary conditions the reader is referred to Roy et al. [64].

Considering the flow on bearing raceways the material of the solid surfaces is steel and the liquid is oil or base oil. Jacod et al. [40] analyzed the reflow of oil onto the running track from the sides under starved conditions. They showed that the effect of the van der Waals energy between the metal surface and an oil layer on the layer thickness profile is insignificant. Therefore, in this work the effect of the van der Waals energy are neglected.

Kuznetsov and Martynov [46] have measured the angle of the surface at the contact line of oil drops on a smooth steel surface in equilibrium. They have found that the contact angles are small, namely approximately 25 degrees at $20^{\circ} \mathrm{C}$ to 1 degree at $180^{\circ} \mathrm{C}$. This means that, especially at high temperatures, the wetting of the steel surface is favorable and oil tends to spread over the surface. Therefore, it is justified to assume that the steel surface in bearings is fully wetted.

For liquid-solid systems with a low wettability, additional relations are required in the model for the behavior of the boundaries between the wetted and the dry areas. A major complication in that case is that the motion of these boundaries, i.e. the contact lines, is conflicting with the no-slip boundary condition. This is referred to as the contact line paradox. There are several methods to overcome this problem. The most simple and practical method is the precursor film model, in which dry areas are modeled as wetted, but with a very thin film of constant thickness, which is called a precursor film. Other complications are for example the occurrence of contact line hysteresis and the breakdown of the validity of the lubrication approximation in the region near the contact line for large contact angles. For more details the reader is referred to Diez et al. [24] and Snoeijer [63]. As the raceway is completely wetted the contact line dynamics are not included in the model. At the edges of the considered domain on the surface, conditions need to be prescribed, such as an absorbing boundary condition or a condition that determines the amount of lubricant flowing into the domain. For bearing raceways these boundary conditions will be discussed in the examples in section 2.3. 


\subsubsection{Scaled body force, pressure and flow velocity}

The body force components (Eq. (2.10)) are written in terms of the dimensionless variables, $\bar{Z}^{\prime}=-\bar{r} \bar{\kappa}_{\theta}$ is substituted, and after multiplication with $H^{2} /(U \mu)$ the dimensionless body force components are:

$$
\begin{aligned}
& \bar{f}_{s}=\frac{\rho \Omega^{2} L H^{2}}{U \eta} \bar{r}^{\prime} \bar{r}\left(1-\varepsilon \bar{\kappa}_{\theta} \bar{n}\right)+2 \frac{\rho \Omega H^{2}}{\eta} \bar{v}_{\theta} \bar{r}^{\prime}, \\
& \bar{f}_{\theta}=-\frac{\rho \Omega H^{2}}{\eta}\left(2 \bar{r}^{\prime} \bar{v}_{s}-2 \varepsilon \bar{r} \bar{\kappa}_{\theta} \bar{w}\right), \\
& \bar{f}_{n}=-\frac{\rho \Omega^{2} L H^{2}}{U \eta} \bar{\kappa}_{\theta} \bar{r}^{2}\left(1-\varepsilon \bar{\kappa}_{\theta} \bar{n}\right)-2 \frac{\rho \Omega H^{2}}{\eta} \bar{v}_{\theta} \bar{r} \bar{\kappa}_{\theta} .
\end{aligned}
$$

The terms of Eq. (2.24) multiplied by $\Omega^{2}$ and $\Omega$ represent the centrifugal forces and the Coriolis forces, respectively. When the centrifugal force is the driving force of the flow, the dimensionless terms of the centrifugal force are of the leading order. In that case the typical flow velocity is:

$$
U=\frac{\rho \Omega^{2} L H^{2}}{\eta} .
$$

Hence, the characteristic time becomes:

$$
t_{c}=\frac{L}{U}=\frac{\eta}{\rho \Omega^{2} H^{2}}
$$

Substituting Eq. (2.25) in Eq. (2.24) and using $\delta=\varepsilon^{2} \operatorname{Re}=H^{2} \rho U /(L \eta)=H^{4} \rho^{2} \Omega^{2} / \eta^{2}$ gives:

$$
\begin{aligned}
& \bar{f}_{s}=\bar{r}^{\prime} \bar{r}\left(1-\varepsilon \bar{\kappa}_{\theta} \bar{n}\right)+2 \delta^{\frac{1}{2}} \bar{v}_{\theta} \bar{r}^{\prime}, \\
& \bar{f}_{\theta}=-\delta^{\frac{1}{2}}\left(2 \bar{r}^{\prime} \bar{v}_{s}-2 \varepsilon \bar{r} \bar{\kappa}_{\theta} \bar{w}\right), \\
& \bar{f}_{n}=-\bar{\kappa}_{\theta} \bar{r}^{2}\left(1-\varepsilon \bar{\kappa}_{\theta} \bar{n}\right)-2 \delta^{\frac{1}{2}} \bar{v}_{\theta} \bar{r} \bar{\kappa}_{\theta} .
\end{aligned}
$$

So, the order of magnitude of the body force $\bar{f}_{\theta}$ is equal to $\delta^{\frac{1}{2}}$ and substitution into Eq. (2.15) shows that the order of magnitude of the velocity $\bar{v}_{\theta}$ is also equal to $\delta^{\frac{1}{2}}$. Hence, the Coriolis terms of the first and third expression of Eq. (2.27) are of the order $\delta$ and may therefore be neglected within the approximations of lubrication theory. For further details on the effect of the Coriolis force on axisymmetric rotating thin layer flows the reader is referred to Myers and Lombe [53]. As a consequence of the small-curvature assumption, the $\mathrm{O}(\varepsilon \bar{\kappa})$ terms will also be neglected. The dimensionless force components become:

$$
\bar{f}_{s}=\bar{r}^{\prime} \bar{r}, \quad \bar{f}_{\theta}=-2 \delta^{\frac{1}{2}} \bar{r}^{\prime} \bar{v}_{s}, \quad \bar{f}_{n}=-\bar{\kappa}_{\theta} \bar{r}^{2} .
$$


The dimensionless pressure is obtained by integration of Eq. (2.17) and application of boundary condition Eq. (2.21), yielding:

$$
\bar{p}=\bar{p}_{0}+\varepsilon \bar{f}_{n}(\bar{n}-\bar{h})-C \tilde{\kappa} .
$$

Substitution of the derivative of the pressure with respect to $\bar{s}$ into Eq. (2.15) and double integration with respect to $\bar{n}$, using the boundary conditions (2.18) and (2.20), leads to the following expression for the velocity along the solid surface in $\bar{s}$ direction:

$$
\bar{v}_{s}=\left[\frac{1}{2} \bar{n}^{2}-\bar{n} \bar{h}\right]\left[-\varepsilon \bar{h} \frac{\partial \bar{f}_{n}}{\partial \bar{s}}-\varepsilon \bar{f}_{n} \frac{\partial \bar{h}}{\partial \bar{s}}-C \frac{\partial \tilde{\kappa}}{\partial \bar{s}}-\bar{f}_{s}\right]+\varepsilon\left[\frac{1}{6} \bar{n}^{3}-\frac{1}{2} \bar{h}^{2} \bar{n}\right] \frac{\partial \bar{f}_{n}}{\partial \bar{s}} .
$$

When $\operatorname{Re} \leq 1$ also $\varepsilon \delta^{\frac{1}{2}} \leq \varepsilon^{2}$ and when $\mathrm{Re} \geq 1$ then $\varepsilon \delta^{\frac{1}{2}} \leq \delta$, hence $\varepsilon \delta^{\frac{1}{2}}$ terms are negligible in accordance with lubrication theory. Substitution of Eq. (2.30) into Eq. (2.27) and neglecting $\mathrm{O}\left(\varepsilon \delta^{\frac{1}{2}}, \varepsilon \bar{\kappa}\right)$ terms results in:

$$
\bar{f}_{\theta}=2 \delta^{\frac{1}{2}} \vec{r}\left[\frac{1}{2} \bar{n}^{2}-\bar{n} \bar{h}\right]\left[C \frac{\partial \tilde{\kappa}}{\partial \bar{s}}+\bar{f}_{s}\right] .
$$

Substitution of Eq. (2.31) and the derivative of Eq. (2.29) with respect to $\theta$ into Eq. (2.16), and double integration with respect to $\bar{n}$, using the boundary conditions (2.18) and (2.20), leads to the following expression for the velocity along the surface in $\theta$ direction:

$\bar{v}_{\theta}=\left[\frac{1}{2} \bar{n}^{2}-\bar{n} \bar{h}\right]\left[-\varepsilon \bar{f}_{n} \frac{1}{\bar{r}} \frac{\partial \bar{h}}{\partial \theta}-C \frac{1}{\bar{r}} \frac{\partial \tilde{\kappa}}{\partial \theta}\right]-2 \delta^{\frac{1}{2}} \bar{r}^{\prime}\left[\frac{1}{24} \bar{n}^{4}-\frac{1}{6} \bar{n}^{3} \bar{h}+\frac{1}{3} \bar{h}^{3} \bar{n}\right]\left[C \frac{\partial \tilde{\kappa}}{\partial \bar{s}}+\bar{f}_{s}\right]$.

\subsubsection{Layer thickness equation}

To obtain a differential equation for the layer thickness $\bar{h}(\bar{s}, \theta, \bar{t})$, the equation of continuity (2.13) is integrated across the layer thickness, using the zero-slip condition (Eq. (2.18)) and the kinematic boundary condition (Eq. (2.19)). Using the Leibniz rule of integration, one obtains:

$$
\frac{1}{\bar{r}} \frac{\partial}{\partial \bar{s}}\left(\bar{r} \bar{Q}_{s}\right)+\frac{1}{\bar{r}} \frac{\partial \bar{Q}_{\theta}}{\partial \theta}+\frac{\partial \bar{h}}{\partial \bar{t}}=0
$$

where the leading order terms of $\bar{Q}_{s}$ and $\bar{Q}_{\theta}$ are found by integration of the tangential velocity vector over the layer thickness:

$$
\bar{Q}_{s}=\int_{0}^{\bar{h}} \bar{v}_{s} d \bar{n}, \quad \bar{Q}_{\theta}=\int_{0}^{\bar{h}} \bar{v}_{\theta} d \bar{n} .
$$

Substitution of Eq. (2.22) , Eq. (2.30) and Eq. (2.32) into Eq. (2.34) and neglecting the $\varepsilon \bar{\kappa}$ terms gives: 


$$
\begin{aligned}
& \bar{Q}_{s}=\frac{1}{3} \bar{h}^{3}(\overbrace{\overline{f_{s}}+\varepsilon \overline{f_{n}} \frac{\partial \bar{h}}{\partial \bar{s}}}^{\text {Centrifugal force }}+\overbrace{C\left(\frac{d \bar{\kappa}}{d s}+\varepsilon \frac{\partial}{\partial \bar{s}}\left(\nabla^{2} \bar{h}\right)\right)}^{\text {Surface tension }}), \\
& \bar{Q}_{\theta}=\frac{1}{3} \bar{h}^{3}(\overbrace{-\frac{4}{5} \delta^{\frac{1}{2}} \bar{r}^{\prime}\left(C \bar{\kappa}^{\prime}+\bar{r}^{\prime} \bar{r}\right) \bar{h}^{2}}^{\substack{\text { Coriolis } \\
\text { force }}}+\overbrace{\varepsilon \bar{f} \frac{1}{\bar{r}} \frac{\partial \bar{h}}{\partial \theta}}^{\substack{\text { Centrifugal } \\
\text { force }}}+\frac{\text { Surface tension }}{\varepsilon C \frac{1}{\bar{r}} \frac{\partial}{\partial \theta}\left(\nabla^{2} \bar{h}\right)}) .
\end{aligned}
$$

Equation (2.35) substituted into Eq. (2.33) leads to a fourth-order partial differential equation for the layer thickness $\bar{h}(\bar{s}, \theta, \bar{t})$, that can be solved numerically, e.g. using a finite-difference method, as is explained in appendix A.2. However, using some additional assumptions that are realistic for the case of thin layer flow on bearing raceways, a simplified equation can be derived from which, given an initial layer, an analytic solution for the layer variation as a function of space and time can be obtained.

\subsubsection{Simplification and analytical approach}

For a bearing application it is justified to assume that, after running in at steady operating conditions, the layer flow is axisymmetric. In Chapter 3 the justification of this assumption will be discussed in more detail. Assuming oil as liquid, the order of magnitude of the surface tension coefficient is $\sigma_{0}=10^{-2} \mathrm{~N} / \mathrm{m}$ and the density will be $\rho=\mathrm{O}\left(10^{3}\right) \mathrm{kg} / \mathrm{m}^{3}$. If the rotational speed is $\Omega>\mathrm{O}\left(10^{3}\right) \mathrm{rpm}=\mathrm{O}\left(10^{2}\right) \mathrm{rad} / \mathrm{s}$ and the geometry of the solid surface is $L>\mathrm{O}\left(10^{-2}\right) \mathrm{m}$, which is reasonable for many applications, the inverse capillary number is small: $C=\sigma_{0} /\left(\rho \Omega^{2} L^{3}\right)=\mathrm{O}\left(10^{-3}\right) \ll 1$. Hence, considering a relatively smooth liquid/air surface, the surface tension terms in Eq. (2.35) can be neglected. Furthermore, when the geometry of the raceway surface is such that $\varepsilon \bar{\kappa}_{\theta} \bar{r} \ll \bar{r}^{\prime}$, the normal force term of Eq. (2.35) is also negligible. For the raceways of tapered roller bearings this is generally the case. For spherical raceways this assumption is violated on a part of the domain. However, as will be shown in section 2.5, this has no significant influence on the global solution. For the conditions assumed $\bar{Q}_{s}$ in Eq. (2.35) becomes:

$$
\bar{Q}_{s}=\frac{1}{3} \bar{h}^{3} \bar{f}_{s}
$$

For an axisymmetric layer $\partial \bar{Q}_{\theta} / \partial \theta=0$. Substitution of Eq. (2.36) into the equation of continuity (2.33) leads to the following equation for the layer thickness $\bar{h}(\bar{s}, \bar{t})$ :

$$
\frac{1}{\bar{r}} \frac{\partial}{\partial \bar{s}}\left(\frac{1}{3} \bar{h}^{3} q\right)+\frac{\partial \bar{h}}{\partial \bar{t}}=0,
$$

where $q(\bar{s})=\bar{r} \bar{f}_{s}=\bar{r}^{2} \bar{r}^{\prime}$. This quasi-linear partial differential equation for $\bar{h}(\bar{s}, \bar{t})$ can be solved by the method of characteristics (See Howell [38] and appendix A.1). The parameterization of the characteristics is illustrated in Figure 2.3. The time $\bar{t}$, the position $\bar{s}$, and the layer thickness $\bar{h}$ are parameterized according to:

$$
\bar{t}=\bar{t}(\sigma, \xi), \quad \bar{s}=\bar{s}(\sigma, \xi), \quad \text { and } \quad \bar{h}=\bar{h}(\sigma, \xi) .
$$




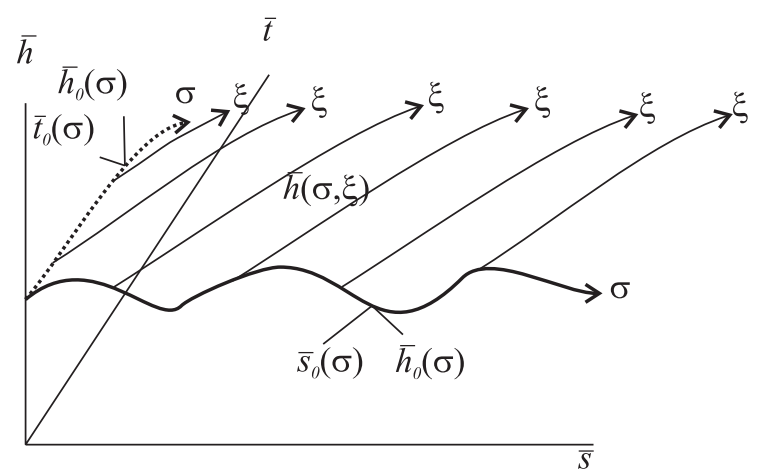

Figure 2.3: 3-D representation of the characteristic curves, parameterized by $\xi$, emerge from the initial curve which is parameterized using $\sigma$.

The characteristic curves, parameterized by $\xi$, emerge from a curve which is parameterized using $\sigma$ :

$$
\xi=0 \quad \rightarrow \quad \bar{t}=\bar{t}_{0}(\sigma), \quad \bar{s}=\bar{s}_{0}(\sigma), \quad \text { and } \quad \bar{h}=\bar{h}_{0}(\sigma) \text {. }
$$

Thus, $\bar{t}_{0}, \bar{s}_{0}$ and $\bar{h}_{0}$ are the starting positions of the characteristic curves on $\bar{t}, \bar{s}$ and $\bar{h}$. The solution of Eq. (2.37) is:

$$
\bar{h}(\sigma, \xi)=\left(\frac{q\left(\bar{s}_{0}\right)}{q(\bar{s})}\right)^{\frac{1}{3}} \bar{h}_{0},
$$

and:

$$
\bar{t}(\sigma, \xi)=\xi+\bar{t}_{0} \Rightarrow \xi=\bar{t}-\bar{t}_{0},
$$

where the general equation for the characteristic projections is:

$$
\int_{\bar{s}_{0}}^{\bar{s}} \frac{\bar{r}(\phi)}{q(\phi)^{\frac{1}{3}}} d \phi=\left(q\left(\bar{s}_{0}\right)\right)^{\frac{2}{3}} \xi \bar{h}_{0}{ }^{2} .
$$

Solving the integral gives the expression for $\bar{s}=\bar{s}(\sigma, \xi)$. The integral only depends on the geometry of the raceway surface, and for some cases, it can be evaluated analytically. In other cases, a suitable analytic approximation may be found. An example of each case is given in the following section. As the simplified modeling equation is hyperbolic and nonlinear, it is clear that in some applications, depending on the initial shape of the liquid surface, a discontinuity in the layer thickness may develop, called a hydraulic jump. Starting with a smooth initial layer may prevent the formation of hydraulic jumps within the spatial domain. In reality the surface tension will provide the physical mechanism by which such shocks are prevented. Including such effects requires solving the complete thin layer equation numerically. 


\subsection{Theoretical results}

In this section the results for the thin layer flow on a tapered and a spherical bearing raceway are presented. These results are obtained with the simplified layer thickness model.

\subsubsection{Tapered raceway}

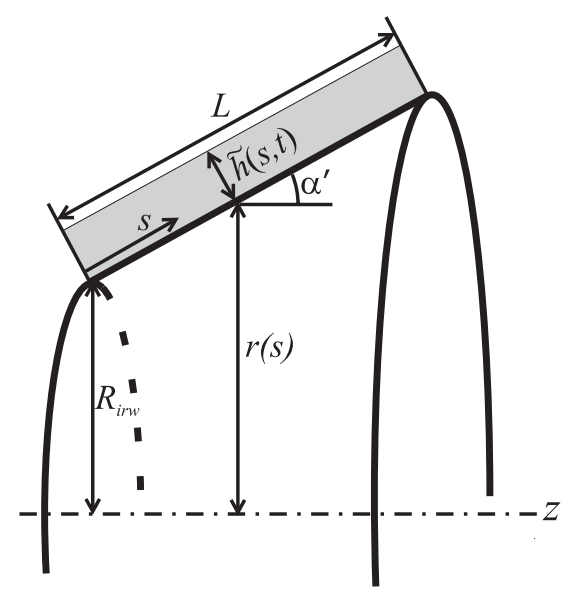

Figure 2.4: Parameterization of the inner raceway of a tapered roller bearing.

The surface is parameterized as shown in Figure 2.4. The non-dimensional radius is given by:

$$
\bar{r}(\bar{s})=R_{i r w} / L+\sin \left(\alpha^{\prime}\right) \bar{s},
$$

The $q$-function becomes:

$$
q(\bar{s})=\bar{r}(\bar{s})^{2} \bar{r}^{\prime}(\bar{s})=\left(R_{i r w} / L+\sin \left(\alpha^{\prime}\right) \bar{s}\right)^{2} \sin \left(\alpha^{\prime}\right) .
$$

Substitution of Eq. (2.41), Eq. (2.43), and Eq. (2.44) into Eq. (2.42) gives the equation for the characteristic projections:

$$
\bar{s}(\sigma, \bar{t})=\left(\frac{4}{3} \sin ^{2}\left(\alpha^{\prime}\right) \bar{h}_{0}^{2}\left(\bar{t}-\bar{t}_{0}\right)+1\right)^{\frac{3}{4}}\left(\frac{R_{i r w}}{L \sin \left(\alpha^{\prime}\right)}+\bar{s}_{0}\right)-\frac{R_{i r w}}{L \sin \left(\alpha^{\prime}\right)} .
$$

Substitution of Eq. (2.44) and Eq. (2.45) into Eq. (2.40) results in:

$$
\bar{h}(\sigma, \bar{t})=\frac{\bar{h}_{0}}{\sqrt{\frac{4}{3} \sin ^{2}\left(\alpha^{\prime}\right) \bar{h}_{0}^{2}\left(\bar{t}-\bar{t}_{0}\right)+1}} .
$$


As an example, in Figure 2.5 the evolution of the layer thickness as a function of location and time is shown for an inner raceway of a tapered roller bearing 30310 (see [2]), with $\alpha^{\prime}=8.9 \mathrm{deg}$ and $R_{\text {irw }} / L=1.69$. The initial layer has a uniform thickness of $\bar{h}_{0}=1$ between $\bar{s}=0$ and $\bar{s}=1$. At $\bar{s}=0$ the layer thickness is zero and no liquid enters the domain. At $\bar{s}=1$ there is an absorbing boundary condition, so the liquid can leave the domain and waves are not reflected. Also, the characteristic projections are shown in the figure.

The layer thickness profile consists of two regions:

Region I: The horizontal line is calculated using Eq. (2.46) with $\bar{t}_{0}(\sigma)=0$, $\bar{h}_{0}(\sigma)=1$, and $\bar{s}_{0}(\sigma)=\sigma$.

Region II: The initial vertical line at $\bar{s}=0$ develops into a curve, which can be obtained using the initial conditions $\bar{t}_{0}(\sigma)=0, \bar{h}_{0}(\sigma)=\sigma$, and $\bar{s}_{0}(\sigma)=0$. For these initial condition Eq. (2.45) and Eq. (2.46) can be written as a single expression:

$$
\bar{h}(\bar{s}, \bar{t})=\sqrt{\frac{3}{4 \sin ^{2}\left(\alpha^{\prime}\right) \bar{t}}\left(1-\frac{1}{\left(\sin \left(\alpha^{\prime}\right) \bar{s}+1\right)^{\frac{4}{3}}}\right)} .
$$

In the long term non-uniform layers also converge to this solution, since the waviness of the liquid surface disappears at $\bar{s}=1$ as a consequence of the absorbing boundary condition. Consequently, Eq. (2.47) is a useful expression to predict the amount of lubricant that is left on a tapered raceways after long times, even when the exact shape of the initial lubricant distribution on the surface is unknown.

The discontinuity in the slope appearing in the layer thickness profiles marks the location of the boundary of region I and II. The characteristic projection of this boundary is represented by a dashed line. It is obtained using Eq. (2.45) with $\bar{t}_{0}=0, \bar{s}_{0}=0$, and $\bar{h}_{0}=1$. 

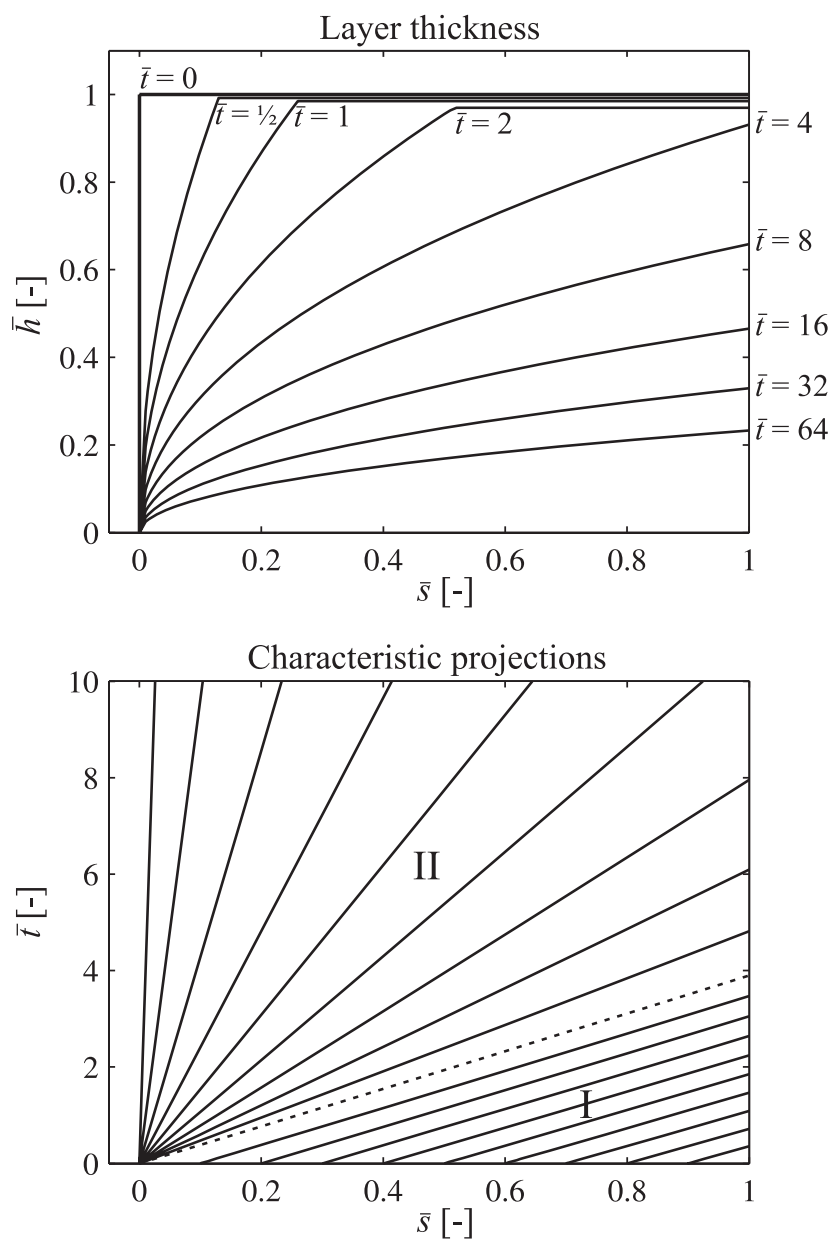

Figure 2.5: Top: Solution layer thickness on a tapered raceway of bearing 30310 in dimensionless variables, starting with a uniform layer. Bottom: characteristic projections. Geometry parameters: $R_{i r w}=31.8 \cdot 10^{-3} \mathrm{~m}, L=18.9 \cdot 10^{-3} \mathrm{~m}$, and $\alpha^{\prime}=8.9 \mathrm{deg}$. 


\subsubsection{Spherical raceway}

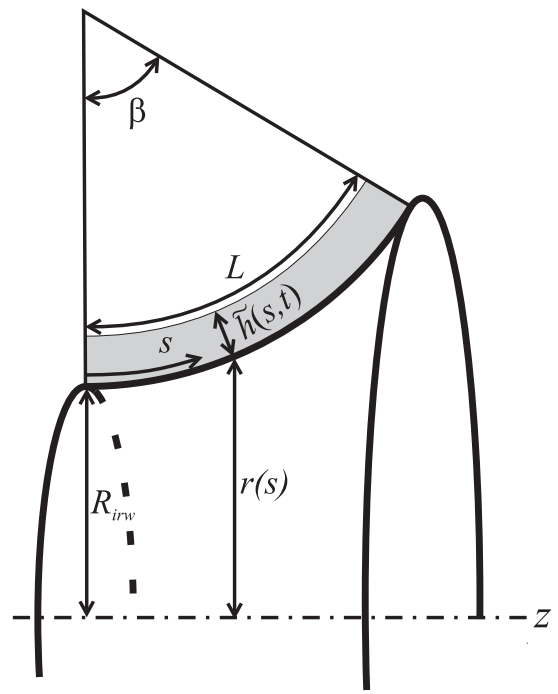

Figure 2.6: Parameterization of the inner raceway of a spherical roller bearing.

The spherical surface is parameterized as shown in Figure 2.6. The dimensionless radius is defined according to:

$$
\bar{r}(\bar{s})=\frac{R_{i r w}}{L}+\frac{1}{\beta}(1-\cos (\beta \bar{s})) .
$$

The $q$-function is defined by:

$$
q(\bar{s})=\bar{r}(\bar{s})^{2} \bar{r}^{\prime}(\bar{s})=\left(\frac{R_{i r w}}{L}+\frac{1}{\beta}(1-\cos (\beta \bar{s}))\right)^{2} \sin (\beta \bar{s}) .
$$

The equations for the characteristic projections $\bar{s}(\sigma, \bar{t})$ is obtained by substituting Eq. (2.41) into Eq. (2.42). The integral is approximated in order to obtain an analytical expression:

$$
\int_{\bar{s}_{0}}^{\bar{s}}\left(\frac{\frac{R_{i r w}}{L}+\frac{1}{\beta}(1-\cos (\beta \phi))}{\sin (\beta \phi)}\right)^{\frac{1}{3}} d \phi \approx \int_{\bar{s}_{0}}^{\bar{s}}\left(\frac{R_{i r w}}{L \beta \phi}\right)^{\frac{1}{3}} d \phi=\left(\frac{R_{i r w}}{\beta L}\right)^{\frac{1}{3}} \frac{3}{2}\left(\bar{S}^{\frac{2}{3}}-\bar{s}_{0}^{\frac{2}{3}}\right) .
$$

When $R_{i r w} / L>1$ and $\beta<0.5$ rad the kernel of the integral of Eq. (2.50) is approximated with a maximal error for $\bar{s}=1$ of $9.1 \%$. 

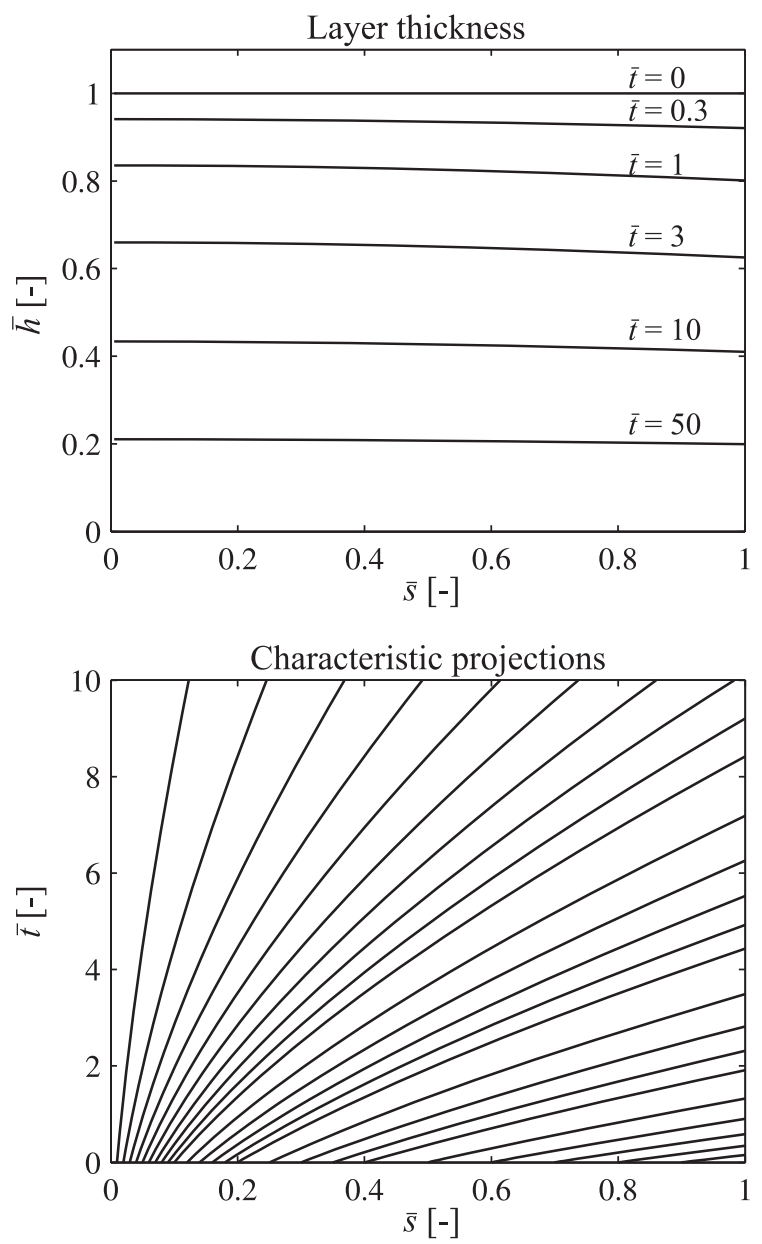

Figure 2.7: Top: Solution layer thickness on the inner raceway of the spherical roller bearing 22205 in dimensionless variables, starting with a uniform layer. Bottom: characteristic projections. Geometry parameters: $R_{i r w}=15.6 \cdot 10^{-3} \mathrm{~m}, L=8.68 \cdot 10^{-3} \mathrm{~m}$, and $\beta=0.36 \mathrm{rad}$.

This results in the following expression for s:

$$
\bar{s}(\sigma, \bar{t})=\left[{\overline{s_{0}}}^{\frac{2}{3}}+\frac{2}{3}\left(\frac{\beta L}{R_{i r w}}\right)^{\frac{1}{3}} \bar{h}_{0}^{2} q\left(\bar{s}_{0}\right)^{\frac{2}{3}}\left(\bar{t}-\bar{t}_{0}\right)\right]^{\frac{3}{2}} .
$$

The layer thickness $\bar{h}(\sigma, \bar{t})$ is defined by Eq. (2.40) with $\xi=\bar{t}-\bar{t}_{0}$. In Figure 2.7 an example is given for the evolution of the layer thickness, as a function of location and time, for the raceway geometry of a spherical roller bearing 22205. Also the characteristic projections are shown in the figure. The reader is referred to the caption for the values of the corresponding parameters. The initial layer and boundary conditions are similar to that 
of the tapered case (part I). Note that the layer uniformity is maintained during outflow. Unlike for the tapered raceway, the tangential flow velocity at $\bar{s}=0$ is zero. This follows from the slope of the solid surface $\vec{r}^{\prime}$ being zero at this boundary.

\subsection{Experimental validation}

To validate the model predictions, the variation of the layer thickness on a rotating raceway surface as a function of time was studied experimentally. In the experiments three different raceway geometries were used: two tapered raceways, with different taper angles, and one spherical raceway. The relevant data is given in Table 2.1. The inner rings of the bearings were mounted on a shaft, driven by an electromotor with adjustable speed.

A layer of oil was distributed on the surface and the layer thickness, as a function of the location (s) at a given angular position, was measured using an optical interferometry surface profiler. As the surface profiler can cover only a limited measuring range (440 microns along the surface), to map the dependence on the position, about 10 measurements were performed at successive locations in $s$ direction along the raceway, as indicated in Figure 2.8. Care was taken that the initial layer was smooth and completely wetted the raceway. Subsequently, the raceway was spun for several intervals of time and the layer thickness was measured at the end of each interval. Since the initial layer may not be completely axisymmetric, it was ensured that each series of measurements was carried out at the same position in circumferential direction.

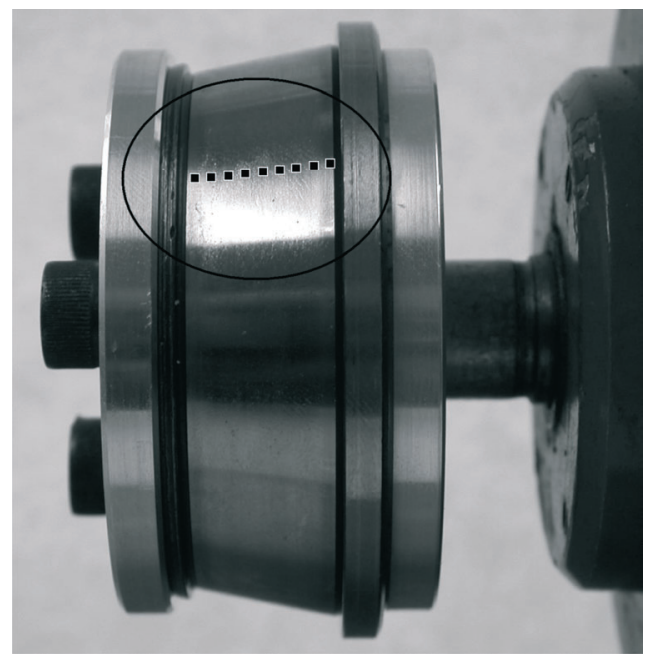

Figure 2.8: Tapered bearing inner raceway mounted on a rotating shaft. The markers indicate locations where layer thickness is measured using the surface profiler. 


\begin{tabular}{|c|c|c|c|c|}
\hline Raceway & Radius $R_{i r w}$ & Length $L$ & Angle & Roughness $R_{a}$ \\
\hline Tapered I & $25.4 \mathrm{~mm}$ & $13.5 \mathrm{~mm}$ & $\alpha^{\prime}=10.9^{\circ}$ & $7 \cdot 10^{-8} \mathrm{~m}$ \\
\hline Tapered II & $28.0 \mathrm{~mm}$ & $16.0 \mathrm{~mm}$ & $\alpha^{\prime}=15.4^{\circ}$ & $14 \cdot 10^{-8} \mathrm{~m}$ \\
\hline Spherical & $27.5 \mathrm{~mm}$ & $10.6 \mathrm{~mm}$ & $\beta=16.3^{\circ}$ & $8 \cdot 10^{-8} \mathrm{~m}$ \\
\hline
\end{tabular}

Table 2.1: Geometry parameters of the raceways.

The entire layer measurement required about 20 minutes. In this time the shape of the liquid layer may have changed by effects of gravity and surface tension. However, these effects are expected to be small, since a high viscosity oil (HVI 650) was used and during measurement the oil layer was positioned horizontally. The experiments were carried out at room temperature, which varied from experiment to experiment between 23 and 25 degrees [C]. The viscosity at room temperature was obtained using the Walther formula, see Seeton [62], using the known viscosity at $40{ }^{\circ} \mathrm{C} \quad(\mu=0.419 \mathrm{~Pa} \cdot \mathrm{s})$ and at $100{ }^{\circ} \mathrm{C}$ $\left(\mu=28.7 \cdot 10^{-3} \mathrm{~Pa} \cdot \mathrm{s}\right)$ as input to determine the two empirical constants in the Walther formula. The oil density used in the calculations is $\rho=910 \mathrm{~kg} / \mathrm{m}^{3}$.

In Figure 2.9 the results of the experiments are shown for the tapered raceway I for three rotational speeds; 500, 1000 and $2000 \mathrm{rpm}$. The results are presented in dimensional variables. In the figure, the sets of dots at different locations $s$ indicate each profile measurement. Results are shown after different times. The drawn line at $t=0$ is a curve fit of the measured initial layer thickness. The other drawn lines in the figure indicate the theoretical predictions. These are obtained using the curve fit of the initial layer thickness as input to the simplified layer thickness model, discussed in section 2.3.

As can be seen from the figure, the results of the model are in good agreement with the measured results, regarding the shape and decay of the layer thickness as a function of time, except in the region just to the left of $s=L$. The observed difference is explained by the fact that in reality the layer experiences a boundary condition at the contact line at $\mathrm{s}=L$. The oil cannot continue to flow in $s$ direction and therefore the oil accumulates, which leads to a region of increased layer thickness. The width of the accumulation region decreases with increasing rotational speed. This implies that the simplified model predictions are accurate for a larger region at higher speeds. The overall layer thickness decays with time according to $\tilde{h} \sim t^{-1 / 2}$, which is in agreement with the model predictions. Comparison of the results for different rotational speeds shows that the relation between the thickness and the rotational speed is given by $\tilde{h} \sim 1 / \Omega$.

The experimental and theoretical results for a speed of $1000 \mathrm{rpm}$ for the tapered raceway type II are shown in Figure 2.10. The results of the measurements for the spherical surface are shown in Figure 2.11. Note that the model predicts an almost uniform layer thickness distribution on a spherical raceway. The measurements confirm this trend, except for the region near the edges. This will be discussed in more detail in the next section. 

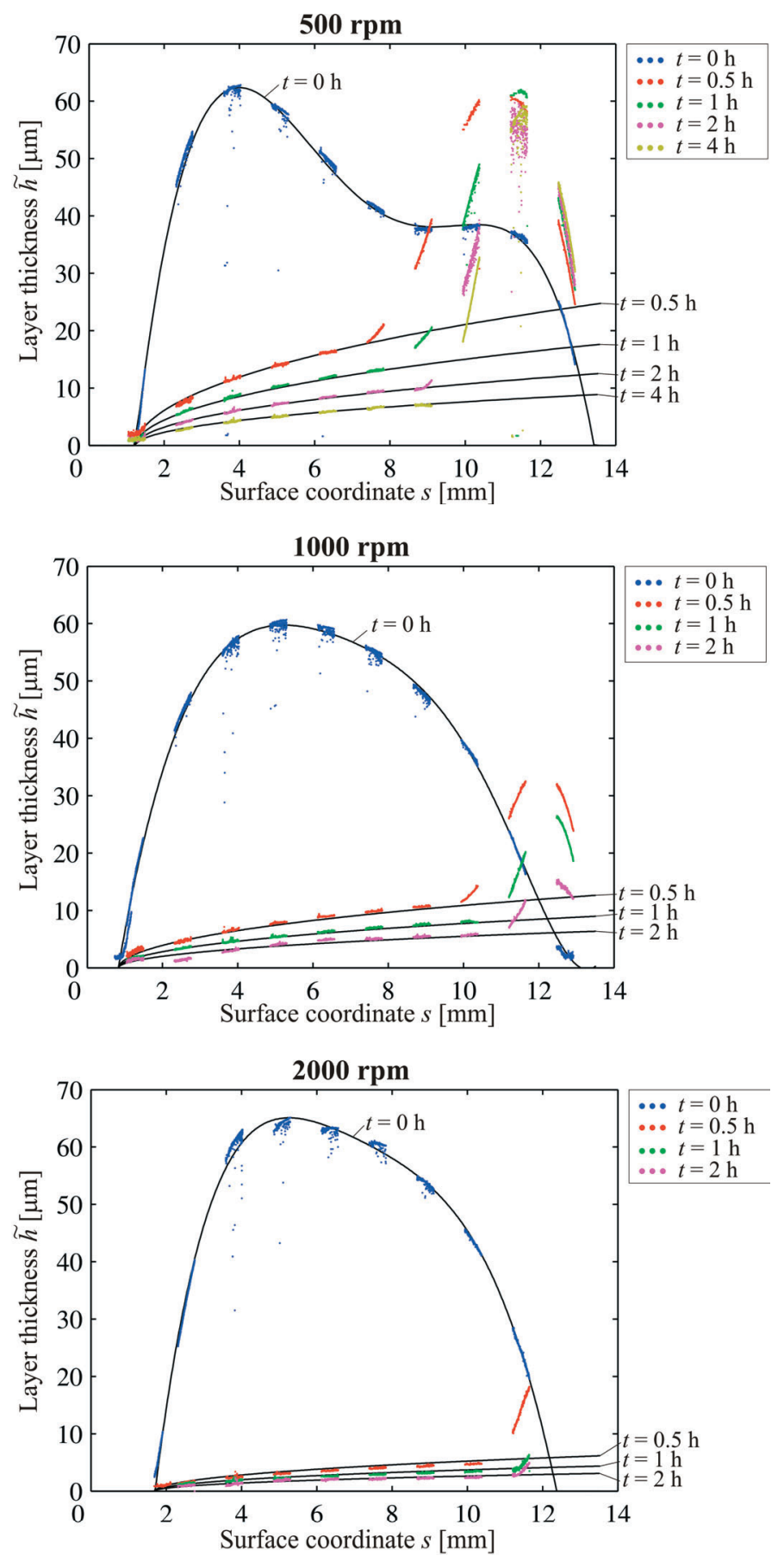

Figure 2.9: Results for the oil layer thickness for different rotational speeds on a tapered surface type I: 500rpm, 1000rpm and $2000 \mathrm{rpm}$, respectively. The dots are the measured values. The drawn lines at $t=0$ are a curve fit of the initial layer. The other drawn lines are predicted by the current model. Viscosity: $\mu=1.25 \mathrm{~Pa} \cdot \mathrm{s}$ at $T=24.6^{\circ} \mathrm{C}$. 


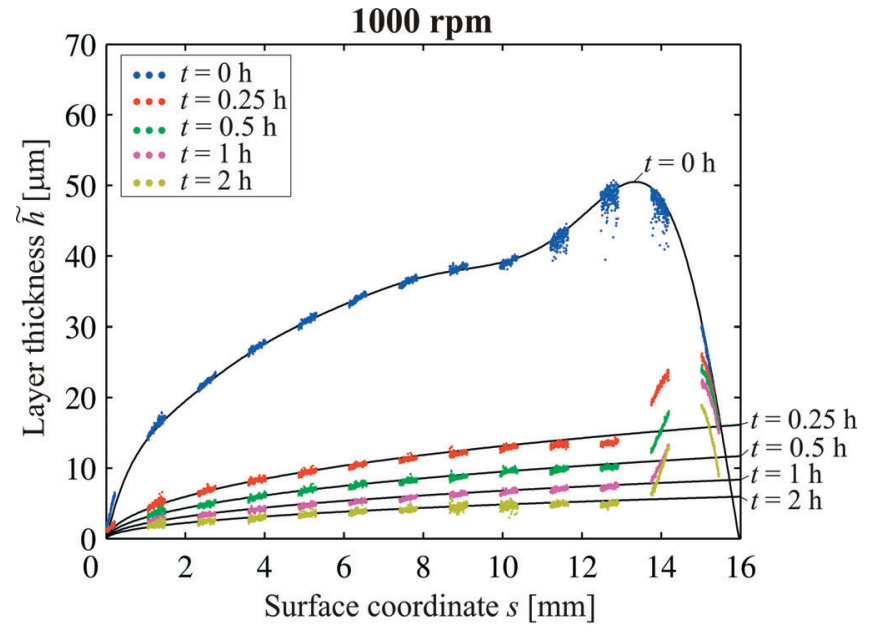

Figure 2.10: Results for the oil layer thickness on the tapered raceway type II rotating at 1000 rpm. Viscosity: $\mu=1.42 \mathrm{~Pa} \cdot \mathrm{s}$ at $T=23.0^{\circ} \mathrm{C}$.

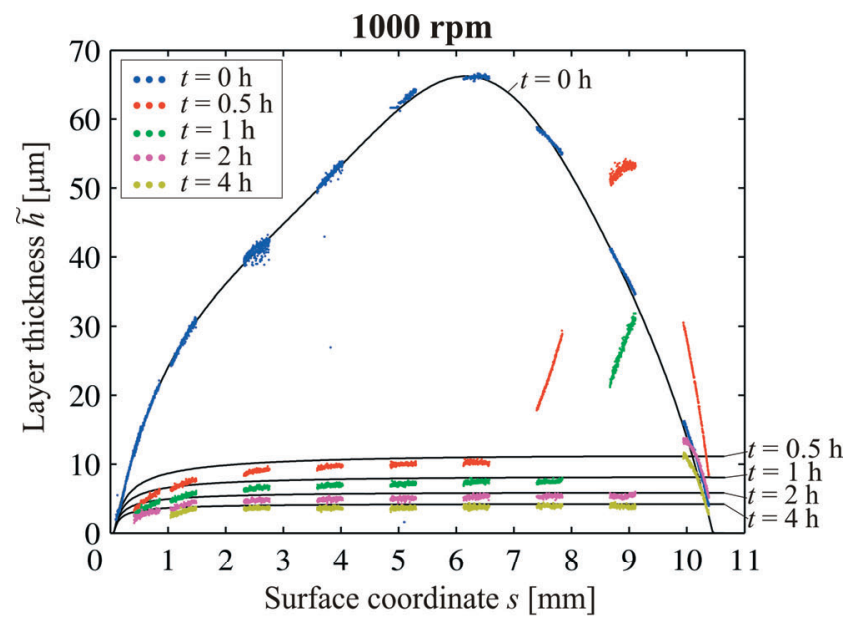

Figure 2.11: Results for the oil layer thickness on the spherical raceway rotating at $1000 \mathrm{rpm}$. Viscosity: $\mu=1.47 \mathrm{~Pa} \cdot \mathrm{s}$ at $T=22.5^{\circ} \mathrm{C}$. 


\subsection{Discussion}

The observed difference between the experimental results and the theoretical results in the edge region can be explained by the fact that edge effects are not included in the simplified model. During the experiments it was observed that near $s=L$ liquid leaves the surface in the form of a droplet. This behavior can not be modeled using the thin layer approximation. Moreover, due to the high curvature and slope of the liquid surface in these areas, the neglected higher-order terms in Eq. (2.35) become significant. Finally, as the geometry of the spherical raceway violates the assumption $\varepsilon \bar{\kappa}_{\theta} \bar{r} \ll \vec{r}^{\prime}$ near $s=0$, where the slope of the raceway $\vec{r}$ tends to zero, the influence of the component of the centrifugal force in $n$ direction is no longer negligible. This results in a difference between the experimental results and the theoretical results in the region near $s=0$.

In order to show the influence of these neglected terms, the predictions of the simplified model have been compared with the results of the numerical solutions of the complete equation for the layer thickness. The reader is referred to appendix A.2 for the discretization of these equations. The parameters of the presented experiment with the spherical raceway are taken as input for the numerical calculations.

The measured layer thickness $\tilde{h}$ is zero at the contact line near $s=L$, hence, according to Eq. (2.35), the liquid flux is zero and thus, in the model no liquid leaves the surface at $s=L$. However, during the experiments it was observed that liquid does leave the surface near this boundary in the form of droplets. Therefore, in order to allow mass to leave the system in the numerical calculations, an extra condition is defined. This condition requires that, when near $s=L$ the positive slope of $\bar{h}$ exceeds a certain critical value, the liquid surface is limited to this maximum slope. In the calculations the surface tension coefficient is assumed to be $\sigma_{0}=4 \cdot 10^{-2} \mathrm{~N} / \mathrm{m}$ and the critical value of the slope is chosen $\partial \bar{h} / \partial \bar{s}=9$, which gives a layer thickness profile $\tilde{h}$ near $s=L$ that roughly resembles the measured one.

In Figure 2.12 the results of the numerically as well as the analytically calculated solutions are shown in terms of dimensional parameters. The scaled spatial step size is $\Delta \bar{s}=1 / 255$ and the scaled time step is $\Delta \bar{t}=2.81 \cdot 10^{-5}$. The reader is referred to appendix A.3 for some comments on the numerical accuracy. Note that near the left and the right boundaries, the higher-order terms indeed influence the solution. Liquid accumulates near $s=L$ and the liquid layer is leveled near $s=0$. However, this does not influence the solution in the central part of the domain, where the solution of the numerical method is in good agreement with the analytical solution. Similar behavior has been observed in the experimental results. 


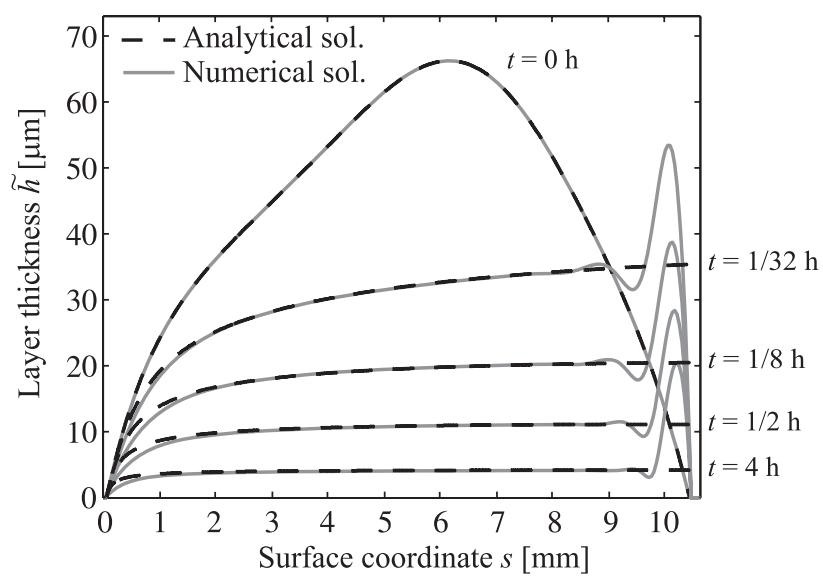

Figure 2.12: Numerical solution of the complete equation (solid lines) and analytical solution of the approximate equation (dashed lines) for the thin layer flow on a spherical raceway rotating at $1000 \mathrm{rpm}$.

\subsection{Conclusion}

In this chapter the effects of the centrifugal force on the flow of an oil layer on a bearing raceway were analysed for different geometries of the raceway. Starting from the NavierStokes equations the free surface thin layer flow equation for axisymmetric rotating solid surfaces was derived. For the case of bearing applications it was shown that, neglecting higher-order terms, a quasi-linear partial differential equation can be derived for the layer thickness, as a function of location and time, which can be solved analytically.

Experiments have been carried out measuring the changes of a layer of oil in time for a tapered and a spherical raceway and for different rotational speeds. It was shown that the simplified model accurately predicts the general trend of layer thickness decrease due to centrifugal effects, except in a region near the boundary, where the effect of the boundary condition on the layer shape is crucial. This was confirmed by the results of numerical simulations, using the complete thin layer flow equations. 


\section{Chapter 3}

\section{CENTRIFUGAL EFFECTS IN ROLLING}

\section{ELEMENT BEARINGS}

The thin layer model presented in Chapter 2 is used to obtain a model for the prediction of the lubricant migration induced by centrifugal effects in rolling element bearings. In particular, the model is used to predict the time variation of the layer thickness as a function of the position across the track on the surface of the inner and outer raceway, and on each of the rollers (in one row) for different types of bearings. The main part of this research has been published in van Zoelen et al. [78].

First, the theoretical formulation of the model is discussed. Subsequently, experimental results are presented, which provide some justification for one of the assumptions made in the development of the model. Finally, as an example the theoretical results for the layer thickness distribution as a function of time are discussed for a spherical roller bearing and a tapered roller bearing.

\subsection{Theoretical formulation}

The derivation of the model in this chapter is presented in dimensional variables. In order to construct a model for the layer evolution in an entire bearing, the thin layer flow on each of

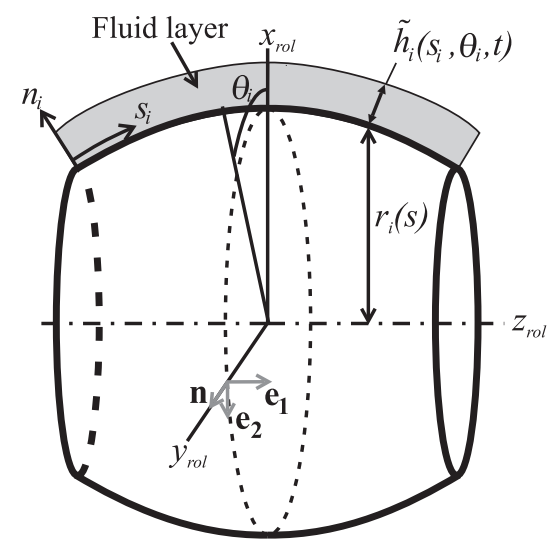

Figure 3.1: A thin liquid layer with a free surface on the surface of a roller and a suitable coordinate system for its description. 
the rollers (in one row) and on the inner and outer raceway is considered. A subscript $i$ will be used to number the solids, i.e. the raceways and the rolling elements in the bearing, see Figure 3.2. The shape of the axisymmetrical surfaces of the rollers and the raceway is defined by the radius function $r_{i}\left(s_{i}\right)$, as was used for the case of a raceway in Figure 2.2. For a roller this is illustrated in Figure 3.1. The flow of the layer on each of the solid surfaces is described using a local orthogonal coordinate system $\left(s_{i}, \theta_{i}, n_{i}\right)$, where $n_{i}$ is the coordinate in the direction of the outward normal to the surface. The thickness of the liquid layer on solid $i$ at a given position $\left(s_{i}, \theta_{i}\right)$ and time $t$ is denoted by $\tilde{h}_{i}\left(s_{i}, \theta_{i}, t\right)$.

\subsubsection{Layer thickness equation}

The continuity equation for an incompressible liquid layer on an axisymmetrical solid surface has been derived in Chapter 2, see Eq. (2.33). Due to the high pressures in the EHL contacts in the bearing, the compressibility of the lubricant may not be neglected. For a compressible liquid layer on solid $i$ the continuity relation averaged over the height of the layer, Eq. (2.33), in dimensional variables reads:

$$
\frac{\partial \rho \tilde{h}_{i}}{\partial t}=-\frac{1}{r_{i}} \frac{\partial}{\partial s_{i}}\left(r_{i} q_{s, i}\right)-\frac{1}{r_{i}} \frac{\partial q_{\theta, i}}{\partial \theta_{i}},
$$

where $q_{s, i}=q_{s, i}\left(s_{i}, \theta_{i}, t\right)$ and $q_{\theta, i}=q_{\theta, i}\left(s_{i}, \theta_{i}, t\right)$, which are the rate of mass flow in the $s_{i}$ direction and in the $\theta_{i}$ direction, respectively, per unit width of the layer. $\rho=\rho\left(s_{i}, \theta_{i}, t\right)$ is the liquid density averaged over the height of the layer.

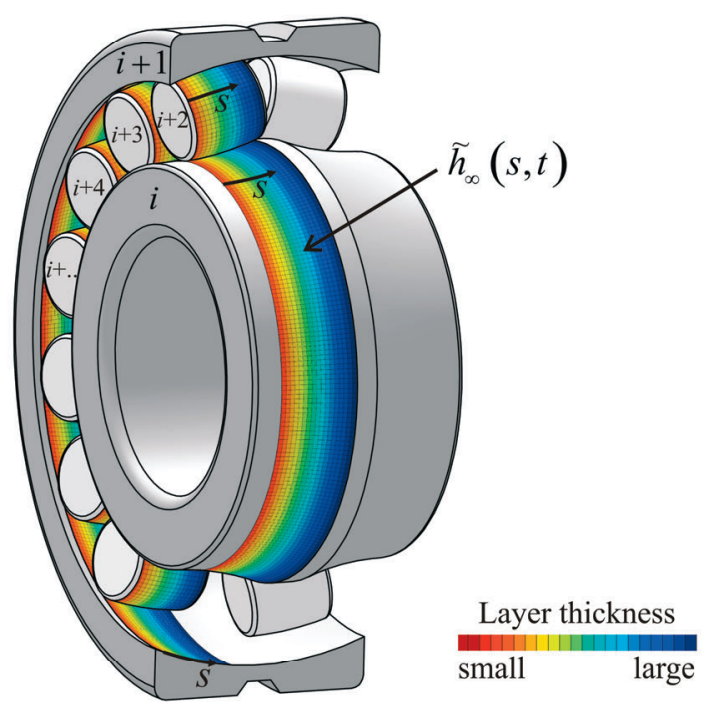

Figure 3.2: Sectional view of a spherical roller bearing. The inner and outer raceway and each of the rollers has its own lubricant layer. 
Next, using Eq. (3.1) a continuity relation will be derived that includes the lubricant layers on the raceways and on each of the rolling elements in one row. Due to the conforming geometry of the solid surfaces of the raceways and the roller in $s_{i}$ direction, a general coordinate $s$ can be used to denote a position across the track on each solid surface, starting at the left boundary of the track, see Figure 3.2.

The layer thickness $\tilde{h}_{i}$ multiplied by $\rho$ and averaged in circumferential direction over all solids, for position $s$ is defined by:

$$
(\rho \tilde{h})_{\infty}=\frac{1}{l_{t}} \sum_{i=1}^{n_{\text {solids }}}\left(r_{i} \int_{0}^{2 \pi} \rho \tilde{h}_{i} d \theta_{i}\right),
$$

where $n_{\text {solids }}$ is the total number of solids in contact, i.e. the inner and the outer raceway and the rolling elements in one row. $l_{t}$ is the total length of the tracks on the surface of these solids:

$$
l_{t}(s)=\sum_{i=1}^{n_{\text {solids }}} 2 \pi r_{i}(s)
$$

Next, Eq. (3.2) is differentiated with respect to time $t$ and Eq. (3.1) is substituted. Also, the term $\partial q_{\theta, i} / \partial \theta_{i}$ is omitted, as its integral with respect to the circumference is zero. This gives:

$$
\frac{\partial(\rho \tilde{h})_{\infty}}{\partial t}=-\frac{1}{l_{t}} \frac{\partial \hat{q}_{s}}{\partial s},
$$

where $\hat{q}_{s}$ is the rate of mass flow in $s$ direction, integrated over the circumference of the solids:

$$
\hat{q}_{s}(s, t)=\sum_{i=1}^{n_{\text {solids }}}\left(r_{i} \int_{0}^{2 \pi} q_{s, i} d \theta_{i}\right) .
$$

The EHL contacts cover only a very small part of the total track length $l_{t}$. Consequently the influence of the density variation in the EHL contacts on the average layer thickness is small and the following approximation is justified:

$$
(\rho \tilde{h})_{\infty} \approx \rho_{0} \tilde{h}_{\infty}
$$

where $\rho_{0}$ is the density at atmospheric pressure and $\tilde{h}_{\infty}=\tilde{h}_{\infty}(s, t)$ is the layer thickness distribution across the track, averaged in circumferential direction over all solids. Substitution of Eq. (3.6) into Eq. (3.4) and division by $\rho_{0}$ gives:

$$
\frac{\partial \tilde{h}_{\infty}}{\partial t}+\frac{1}{l_{t} \rho_{0}} \frac{\partial \hat{q}_{s}}{\partial s}=0 .
$$




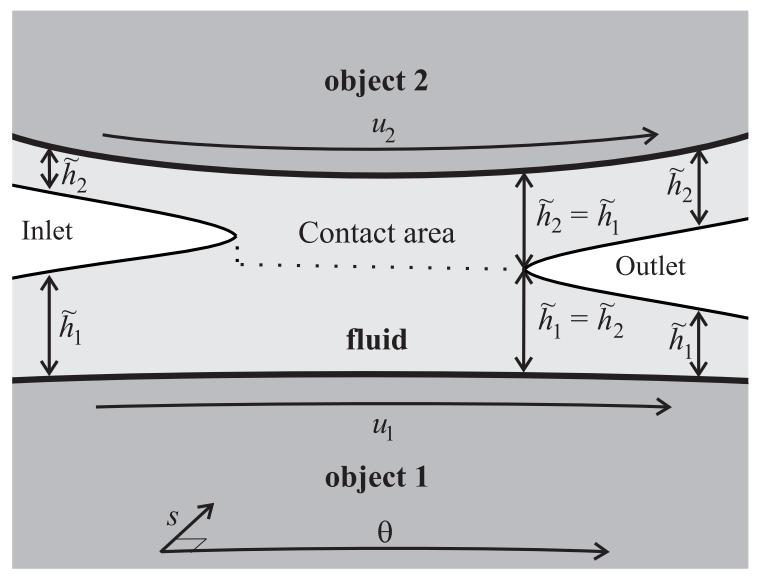

Figure 3.3: Illustration of concept of equipartition: At the outlet of the contact the layer thickness on both solid objects is assumed to be equal.

By using Eq. (3.7) the layer thickness $\tilde{h}_{\infty}$ can be calculated, when the rate of mass flow $\hat{q}_{s}$ is known. The rate of volume flow per unit width of the layer $Q_{s}$ due to the centrifugal effect is defined by Eq. (2.36) in dimensionless variables. Writing this equation in terms of the dimensional variables, see Eq. (2.11), and multiplication by the liquid density $\rho$, the rate of mass flow in s-direction per unit length on the circumference is obtained:

$$
q_{s, i}\left(s, \theta_{i}, t\right)=\rho Q_{s, i}=\frac{\rho \tilde{h}_{i}^{3}}{3 \eta} f_{s, i},
$$

where $\eta$ is the viscosity of the lubricant and $f_{s, i}=f_{s, i}\left(s, \theta_{i}, t\right)$ is the body force component in $s$-direction, that acts on the lubricant layer on solid $i$.

Next, the layer thickness model is simplified by assuming that the layer thickness distribution in s-direction is equal for all solids $i$ and uniform in circumferential direction. This assumption is based on the hypothesis that, in each roller-raceway contact, the lubricant in the exit region equally partitions in two layers on the surfaces of the roller and the raceway. For a single overrolling this is illustrated in Figure 3.3. The continuous exchange of lubricant in the roller raceway contacts, together with other effects such as contact pressure (see Venner et al. [68]) and surface tension, will tend to distribute the lubricant evenly along the circumference of the rollers and the raceways. In addition, the flow speed is low compared to the rotational speed of the bearing. Consequently the change of the layer thickness in between two consecutive roller-raceway contacts can only be small. Therefore, in the long run after many overrollings, the layer thickness distribution will be uniform in circumferential direction and equal for the raceways and the rollers. In Figure 3.2 an example of such a layer thickness distribution is shown. In section 3.2 the experimental validation of this assumption is discussed. 
Accordingly, the layer thickness $\tilde{h}_{i}\left(s, \theta_{i}, t\right)$ of Eq. (3.8) can be replaced by the average layer thickness $\tilde{h}_{\infty}(s, t)$. The EHL contact covers only a very small part of the track, thus the effect of the density and viscosity increase in the EHL contacts on the total mass flow due to centrifugal effects may be assumed to be negligible. Consequently, the value for the ambient viscosity $\eta=\eta_{0}$ and the ambient pressure $p=p_{0}$ can be used. Substitution of Eq. (3.8), $\eta=\eta_{0}, \rho=\rho_{0}$, and $\tilde{h}_{i}=\tilde{h}_{\infty}(s, t)$ into Eq. (3.5) gives:

$$
\hat{q}_{s}(s, t)=\frac{\rho_{0} \tilde{h}_{\infty}^{3}}{3 \eta_{0}} \sum_{i=1}^{n_{\text {solids }}}\left(r_{i} \int_{0}^{2 \pi} f_{s, i} d \theta_{i}\right) .
$$

Substitution of Eq. (3.9) into Eq. (3.7) and rearranging terms gives the final equation for the layer thickness:

$$
\frac{1}{l_{t}} \frac{\partial}{\partial s}\left(\frac{1}{3} \tilde{h}_{\infty}^{3} q\right)+\frac{\partial \tilde{h}_{\infty}}{\partial t}=0,
$$

with:

$$
q(s)=\frac{1}{\eta_{0}} \sum_{i=1}^{n_{\text {solids }}}\left(2 \pi r_{i} \hat{f}_{s, i}\right)
$$

where $\hat{f}_{s, i}$ is the body force component in $s$ direction averaged over the circumference of solid $i$ :

$$
\hat{f}_{s, i}(s)=\frac{1}{2 \pi} \int_{0}^{2 \pi} f_{s, i}\left(s, \theta_{i}, t\right) d \theta_{i} .
$$

Notice that the average body force is only a function of the position s. This will be discussed in more detail in the next section. For convenience of notation the subscript $\infty$ in $\tilde{h}_{\infty}$ will be omitted in the following sections.

\subsubsection{Body forces}

The body forces acting on the lubricant layer due to the centrifugal effects are expressed in terms of a component $f_{n}$, directed in normal direction to the solid surface and a tangential component $f_{s}$. The tangential component is the driving force for the flow (migration) across the track. For a raceway the force components are defined by Eq. (2.28) in dimensionless variables. Writing this equation in terms of the dimensional variables and substitution of $f_{s}$ into Eq. (3.12) gives:

$$
\hat{f}_{s, r w}(s)=\rho_{0} \Omega_{r w}^{2} r_{r w} \frac{d r_{r w}}{d s},
$$

where $\Omega_{r w}$ is the rotational speed of the raceway, $r_{r w}=r_{r w}(s)$ defines the geometry of the raceway, and $\rho_{0}$ is the ambient density of the liquid. 

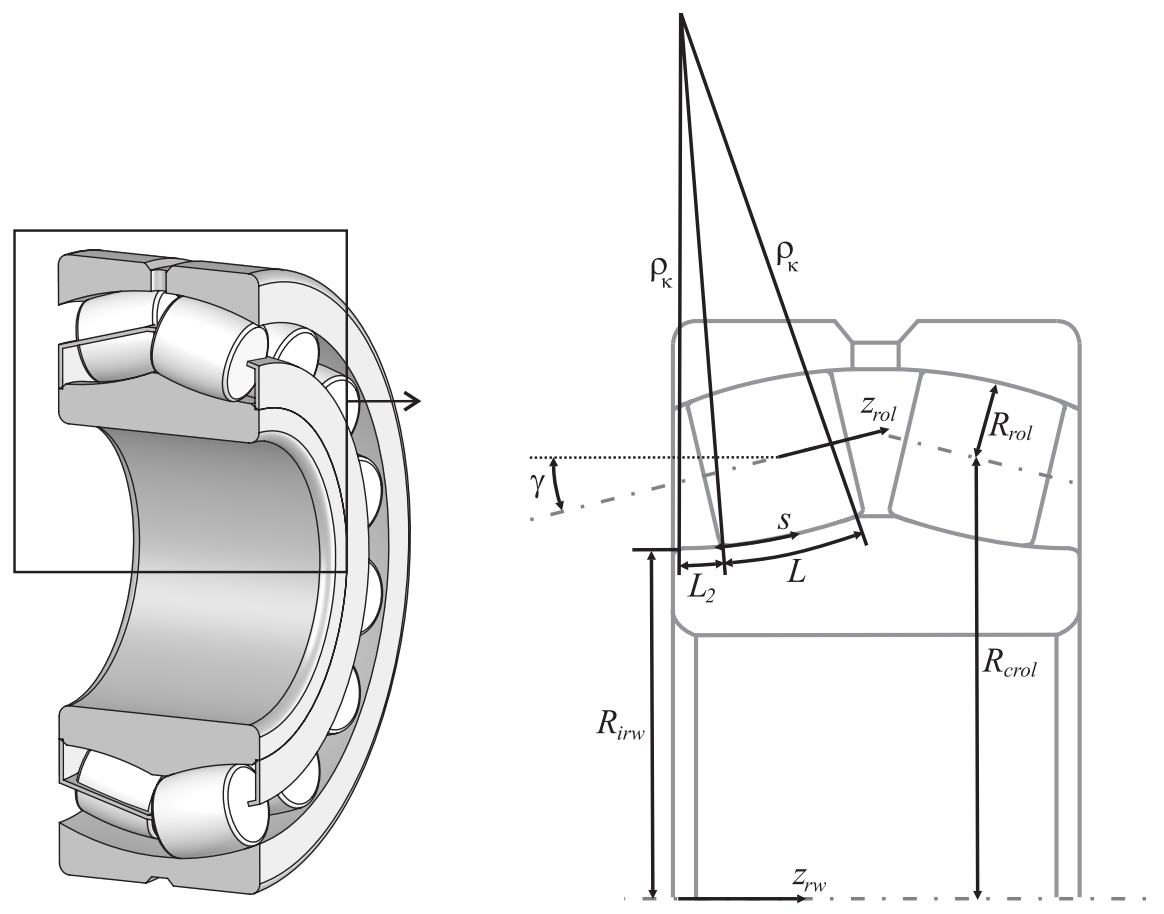

Figure 3.4: Sectional view of a spherical roller bearing including the relevant geometry parameters.

It is assumed that the inner ring of the bearing is rotating and the outer ring is stationary. The rollers rotate around the central axis of the bearing $z_{r w}$ as well as around their own axis of rotational symmetry $z_{\text {rol }}$, see Figure 3.4. In addition, in many configurations, e.g. spherical and tapered roller bearings, these two axes are not aligned. Due to the relatively complex motion of a roller, the body force acting on the layer on this roller is a function of the position $\left(s, \theta_{i}\right)$ and time $t$. For a detailed derivation of these body forces the reader is referred to Appendix B. The time-dependent terms are periodic with a wave length of one rotation of the roller around the $z_{\text {rol }}$ axis. It is found that the equations for the body forces are quite involved. However, after substitution of the body force component $f_{s, \text { rol }}$ into Eq. (3.12) and evaluating the integral, it is simplified substantially and it is only a function of the position $s$ :

$$
\begin{aligned}
\hat{f}_{s, r o l}(s)= & \rho_{0} \Omega_{c a}{ }^{2}\left(\sin ^{2}(\gamma) z_{r o l}+\sin (\gamma) R_{c r o l}\right) \frac{d z_{r o l}}{d s} \\
& +\left(\left(\frac{1}{2} \cos ^{2}(\gamma)+\frac{1}{2}\right) \Omega_{c a}{ }^{2}-2 \Omega_{c a} \Omega_{r o l} \cos (\gamma)+\Omega_{r o l}{ }^{2}\right) \rho_{0} r_{r o l} \frac{d r_{r o l}}{d s},
\end{aligned}
$$

where $\Omega_{c a}$ denotes the rotational speed of the cage of the bearing relative to the fixed outer raceway, $\Omega_{\text {rol }}$ the rotational speed of the roller relative to the cage, $R_{\text {crol }}$ the distance between the center of the roller and the rotational axis of the bearing, and $\gamma$ the angle 
between the axis of rotation of the roller and the cage, see Figure 3.4. $r_{\text {rol }}=r_{\text {rol }}(s)$ defines the geometry of the roller and $z_{\text {rol }}(s)$ is defined as:

$$
Z_{\text {rol }}(s)=\int_{\frac{1}{2} L}^{s} \sqrt{1-\left(\frac{d r_{\text {rol }}}{d s}\right)^{2}} d \phi
$$

\subsubsection{Solution layer thickness equation}

Equation (3.10) is an hyperbolic equation that can be solved using the method of characteristics, see appendix A.1. The solution for the layer thickness can be expressed as:

$$
\tilde{h}(s, t)=\left(\frac{q\left(s_{0}\right)}{q(s)}\right)^{\frac{1}{3}} \tilde{h}_{0} .
$$

The equation for the characteristic projections is defined by:

$$
\int_{s_{0}}^{s} \frac{l_{t}(\phi)}{(q(\phi))^{\frac{1}{3}}} d \phi=\left(q\left(s_{0}\right)\right)^{\frac{2}{3}}\left(t-t_{0}\right) \tilde{h}_{0}{ }^{2},
$$

where $t_{0}, s_{0}$ and $\tilde{h}_{0}$ define the starting position of the characteristics in the solution space $t, s$ and $\tilde{h}$. The boundary conditions will be discussed in the examples at the end of this chapter.

Equation (3.17) can easily be evaluated numerically. In this study a recursive adaptive Simpson quadrature approximation to the integral was used, with an absolute error tolerance of $10^{-6}$. The resulting equation was evaluated iteratively with a termination tolerance of approximately $2 \cdot 10^{-16}$.

\subsubsection{Independent parameters}

When pure rolling contacts are assumed, the rotational speeds of each of the solids $i$ is linearly related to a reference rotational speed $\Omega$. All variables that describe the geometry of the solid surfaces, like $r_{i}, z_{i}, s$ and $l_{t}$, can be scaled using the same coefficient $L$. The scaled variables will be denoted by a bar. From Eq. (3.13) and Eq. (3.14) it follows that the body force is linearly dependent on $f / \bar{f}=\rho_{0} \Omega^{2} L$. Subsequently, from Eq. (3.11), it is deduced that $q / \bar{q}=\rho_{0} \Omega^{2} L^{2} / \eta$. When the layer thickness is scaled by $\tilde{h}=H \bar{h}$ and the time by $t=\tau_{c} \bar{t}$, the layer thickness equation (3.10) written in dimensional variables reads:

$$
\frac{\rho_{0} \Omega^{2} H^{3}}{\eta} \frac{1}{\bar{l}} \frac{\partial}{\partial \bar{s}}\left(\frac{1}{3} \bar{h}^{3} \bar{q}\right)+\frac{H}{\tau_{c}} \frac{\partial \bar{h}}{\partial \bar{t}}=0 .
$$

Multiplication of Eq. (3.18) by $\tau_{c} / H$ then leaves a single dimensionless group of parameters, which is unity, when the time scale $\tau_{c}$ is defined as given in Eq. (2.26), i.e. $\tau_{c}=\eta /\left(\rho \Omega^{2} H^{2}\right)$. According to this time scale, the effect of the centrifugal force on the 
layer thickness is larger for thick layers of low viscosity and high density, rotating at high speeds.

Note that the size scaling parameter $L$ does not appear in the scaled layer thickness equation (3.18). This implies that the model will give the same results for identical bearings of different size, where identical refers to all aspects identical including the shape of the lubricated surfaces, number of rollers, etc. So, in addition to the time scale $t_{c}$ the solution for $\tilde{h}(s, t)$ depends on the number of rollers and the parameters that define the shape of the surfaces of the inner and outer raceway and the rollers.

\subsection{Experimental validation}

In section 3.1.1 it has been conjectured that the lubricant layer partitions in two equal layers in the exit region of each roller-raceway EHL contact, one on the roller and one on the raceway, see Figure 3.3. This assumption is not crucial in the model, as a different partitioning can be used, although this will give different results. When the surface properties such as wettability and roughness are not very much different, assuming equipartition appears reasonable, certainly upon average in the long run. In the long run after many equipartitions, the layer thickness distribution will be uniform in circumferential direction and equal for the raceways and the rollers.

In this section direct experimental evidence for the occurrence of equipartition is presented. In the experiments a wetted plate is overrolled by a ring, at low speed. Subsequently, the thickness of the oil layer on the ring and that on the plate are measured, showing that the lubricant equally partitions between the steel surfaces. Additional (indirect) evidence for the occurrence of equipartition in a starved EHL contacts, also at high speeds and during many overrollings, is provided in Chapter 5. The layer thickness model presented in Chapter 5 is also based on the assumption of equipartition and uniform layers along the track. This model is experimentally validated and the results show good agreement between theory and experiment. The reader is referred to Chapter 5 for more details.

\subsubsection{Experimental setup and procedure}

The experimental setup consists of a steel ring, representing a roller, and a steel flat plate, representing the raceway, with four grooves of different depth, i.e. approximately 5, 10, 20 and $40 \mu \mathrm{m}$ (see Figure 3.5). At the start of the experiment, the steel surfaces are thoroughly cleaned. One of the grooves is completely filled with oil, which is subsequently carefully levelled off, in order to get a smooth layer with at least the thickness of the depth of the groove. The clean ring is aligned and manually rolled over the groove, making a single revolution with a speed of approximately $0.02 \mathrm{~m} / \mathrm{s}$. Subsequently, the thickness of the oil layer picked up on the ring and of the layer that remained behind in the groove is measured using optical interferometry.

The depth of the grooves is chosen such that the resulting thickness of the oil layers is in the measurement range of the optical profiler, which is approximately 1-70 $\mu \mathrm{m}$. The measurements are carried out at four locations along the groove $20 \mathrm{~mm}$ apart. The corresponding positions of the measurements on the ring and the groove are chosen such 
that they are in contact simultaneously during the experiment. The layers are measured at the center of the groove and the ring circumference, respectively, in order to minimize edge effects. The experiment is carried out three times for each groove, each time starting with a clean ring and a newly lubricated groove.

The ring used is the inner raceway of a cylindrical roller bearing and has an outer diameter of $26.5 \mathrm{~mm}$. The width of the grooves and of the ring is $6.3 \mathrm{~mm}$ and $19 \mathrm{~mm}$, respectively. On average the surface roughness of the steel surfaces was $0.4 \mu \mathrm{m}$. The measured RMS surface roughness of the steel surfaces did not exceed $14 \%$ of the depth of any groove. The experiment is done at a room temperature of $21{ }^{\circ} \mathrm{C}$. Oil with a high viscosity (HVI650) is used, in order to minimize changes of the layer thickness during the optical measurements.

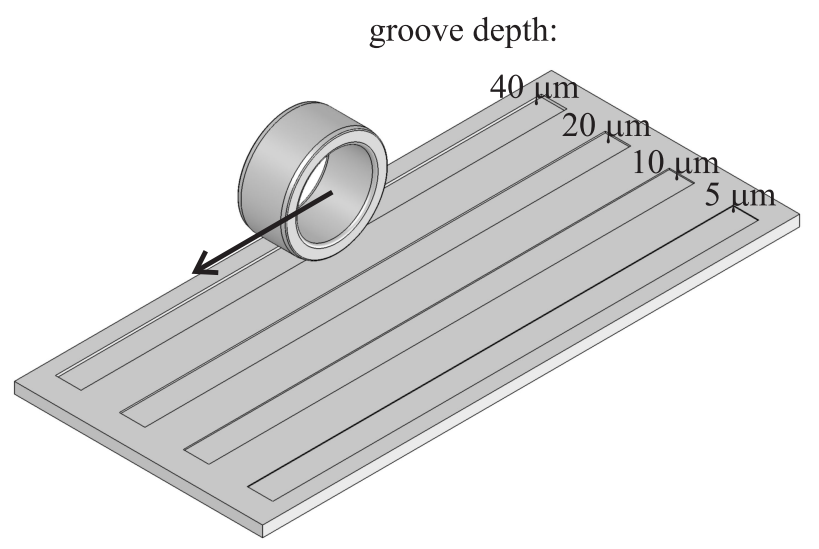

Figure 3.5: Experimental setup. A steel flat plate with four grooves of different depth. The clean ring is manually rolled over a groove, which is filled with oil. 


\subsubsection{Experimental results}

The results of the layer thickness measurements are presented in Figure 3.6. Note that the layer indeed, for the bulk of the results, equally partitions between the groove and the raceway. The results show a slight decreasing trend of the relative thickness of the oil layer on the ring with groove depth. This may be attributed to gravity affecting the layer thickness during the rolling and the measurement procedure, which has a larger effect on thicker layers. The series of measurement results, denoted by * and $\mathrm{x}$ for the groove depths of 10 and $20 \mu \mathrm{m}$, respectively, show a large deviation from the expected value. This can be explained by analysis of the initial layer thickness. It was found that for these measurements the groove was not properly filled with oil before overrolling. The initial layer thickness was only $35 \%$ to $75 \%$ of the depth of the groove. This results in the entrapment of air in the contact, which explains the smaller layer thickness on the ring.

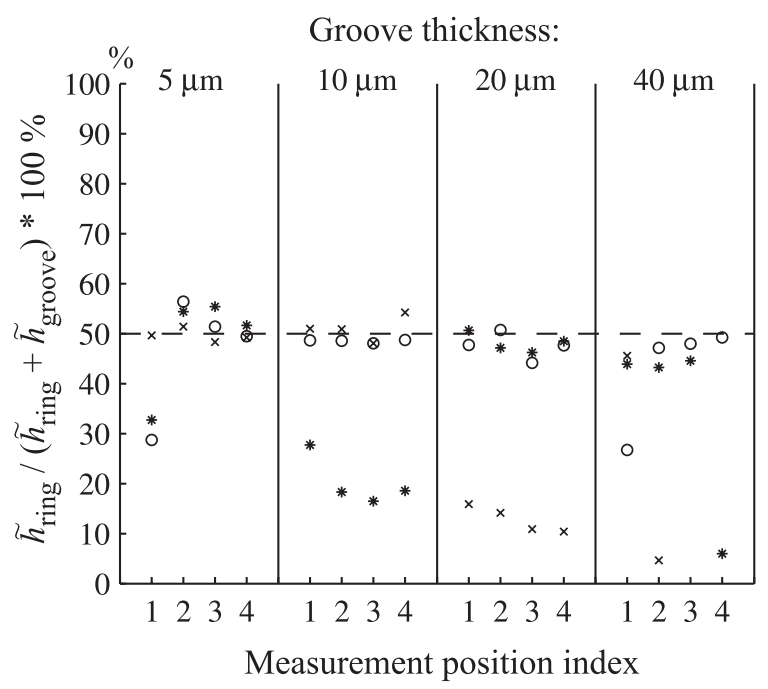

Figure 3.6: Measurement results showing the percentage of the lubricant situated on the ring after rolling over the grooves. The experiment has been repeated three times for every groove, denoted by $\mathrm{o}, \mathrm{x}$ and *. 


\subsection{Example 1: spherical roller bearing}

As a first example, the model is applied to a spherical roller bearing. In the following sections the equations for the geometry of the rollers and the raceways, the boundary conditions, and the independent parameters are specified for this bearing. Finally, the results for the film thickness distribution are discussed.

\subsubsection{Geometry}

The parameters used to describe the spherical roller bearing are shown in Figure 3.4 on page 38. It is assumed that the shape of the raceway and the rollers in s-direction is an arc. The radius function $r(s)$ of the inner raceway, the rollers and the outer raceways are defined as:

$$
\begin{aligned}
& r_{i r w}(s)=R_{i r w}+\rho_{\kappa}-\rho_{\kappa} \cos \left(\frac{L_{2}+s}{\rho_{\kappa}}\right), \\
& r_{r o l}(s)=R_{r o l}-\rho_{\kappa}+\rho_{\kappa} \cos \left(\frac{\frac{1}{2} L-s}{\rho_{\kappa}}\right),
\end{aligned}
$$

and:

$$
r_{\text {orw }}(s)=r_{i r w}(s)+2 \cos (\gamma) r_{\text {rol }}(s) \text {, }
$$

respectively. The axial position on the roller is defined as:

$$
Z_{\text {rol }}(s)=-\rho_{\kappa} \sin \left(\frac{\frac{1}{2} L-s}{\rho_{\kappa}}\right) .
$$

The total track length is defined as:

$$
l_{t}(s)=2 \pi r_{i r w}(s)+2 \pi r_{\text {orw }}(s)+n_{r} 2 \pi r_{\text {rol }}(s) .
$$




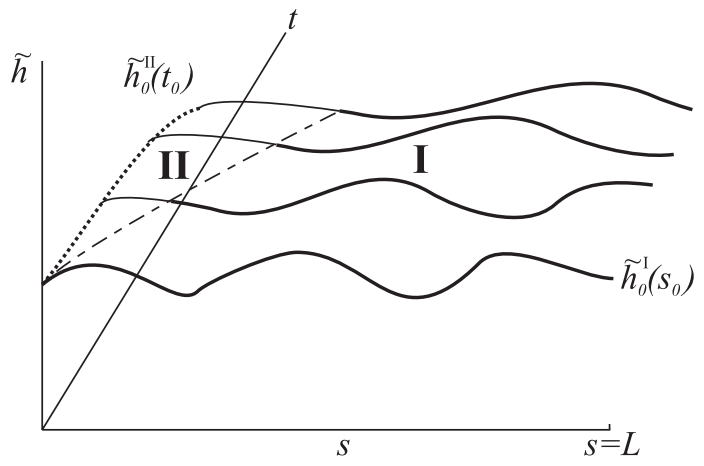

Figure 3.7: Solution of the layer thickness with initial condition and boundary condition.

\subsubsection{Initial and boundary conditions}

In Figure 3.7 a schematic representation of a solution for $\tilde{h}$ is shown. The solution consists of two domains, I and II, which are separated by the dashed line. Liquid is assumed to flow into the domain at $s=0$ and to leave domain at the opposite boundary on the right at $s=L$. The solution in region I originates from the layer thickness distribution at time $t=0$. The solution in region II originates from the inflow at $s=0$. The starting positions of the characteristics for region I and II are, respectively:

Region I: $\tilde{h}_{0}=\tilde{h}_{0}^{I}\left(s_{0}\right), t_{0}=0$ and $s_{0}$ is found by solving Eq. (3.17) for a given $s$ and $t$,

Region II: $\tilde{h}_{0}=\tilde{h}_{0}^{I I}\left(t_{0}\right), s_{0}=0$ and $t_{0}$ is found by solving Eq. (3.17) for a given $s$ and $t$,

where $\tilde{h}_{0}^{I}\left(s_{0}\right)$ is the initial layer thickness distribution as a function of the position $s=s_{0}$ and $\tilde{h}_{0}^{I I}\left(t_{0}\right)$ is the layer thickness as a function of time $t=t_{0}$ at the position of the inflow $s=0$. The location of the boundary between domain I and II at time $t$ is found by solving Eq. (3.17) for $s$, with $h_{0}=h_{0}(0), s_{0}=0$ and $t_{0}=0$. When no liquid leaves or enters the domain at $s=L$ a third domain exists with the following starting positions of the characteristics:

Region III: $s_{0}=L, t_{0}=0$ and $h_{0}$ is found by solving Eq. (3.17) for a given $s$ and $t$.

The location of the boundary between domain II and III at time $t$ is found by solving Eq. (3.17) for $s$, with $h_{0}=h_{0}(L), s_{0}=L$, and $t_{0}=0$. 


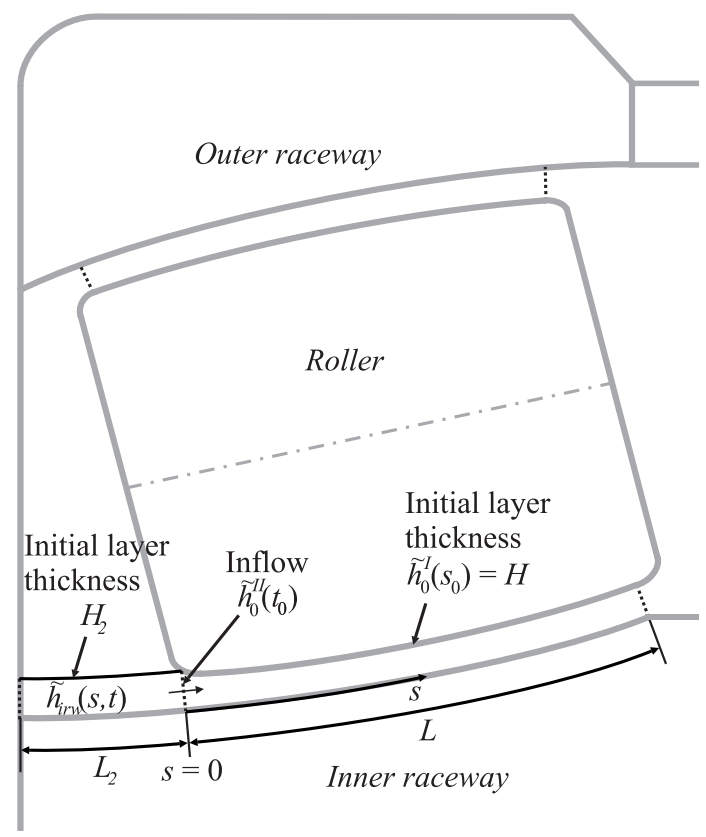

Figure 3.8: Sectional view of a spherical roller bearing including the initial and inflow conditions.

In this example the initial layer thickness distribution is defined by:

$$
\tilde{h}_{0}^{I}\left(s_{0}\right)=H \text {, }
$$

with $H$ a constant. The layer thickness at the inflow $\tilde{h}_{0}^{I I}\left(t_{0}\right)$ is determined by the amount of lubricant flowing onto the track from the side. This is illustrated in Figure 3.8. Substitution of Eq. (3.7) into Eq. (3.10) it is deduced that:

$$
\hat{q}_{s}(s, t)=\frac{1}{3} \rho_{0}(\tilde{h}(s, t))^{3} q(s) .
$$

Rewriting Eq. (3.25) for the layer thickness and substitution of $s=0$ the layer thickness at the position of the inflow is found by:

$$
\tilde{h}_{0}^{I I}\left(t_{0}\right)=\left(\frac{3 \hat{q}_{s}\left(0, t_{0}\right)}{\rho_{0} q(0)}\right)^{1 / 3},
$$

where $\hat{q}_{s}\left(0, t_{0}\right)$ is the rate of mass flow at $s=0$, flowing onto the track from the side as a function of time $t_{0}$. From the perspective of grease lubrication it represents a "grease bleeding rate" parameter, which may be defined as a function of time. In this study, this mass flow rate will be related to the flow rate due to centrifugal effects of an initially uniform oil layer with thickness $H_{2}$, which is situated in the region of the inner raceway to 
the side of the roller, denoted by $L_{2}$, see Figure 3.8. This mass flow rate is obtained by integration of Eq. (3.8) over the circumference of the inner raceway and substitution of Eq. (3.13):

$$
\hat{q}_{s}(0, t)=2 \pi \rho_{0} \Omega_{i r w}^{2} r_{i r w}^{2} \frac{d r_{i r w}}{d s} \frac{\rho_{0}\left(\tilde{h}_{i r w}(0, t)\right)^{3}}{3 \eta_{0}} .
$$

The layer thickness $\tilde{h}_{i r w}(0, t)$ can be calculated starting with Eq. (3.16), with $s=0$, $q(s)=2 \pi r_{i r w} \hat{f}_{s, i r w} / \eta_{0}=2 \pi \rho_{0} \Omega_{i r w}^{2} r_{i r w}^{2}\left(d r_{i r w} / d s\right) / \eta_{0}$ and $l_{t}=2 \pi r_{i r w}$. The starting point of the characteristics $s_{0}$ is found by solving Eq. (3.17), where the integral of Eq. (3.17) can be simplified to:

$$
\begin{aligned}
& \int_{s_{0}}^{0}\left(\frac{l_{t}(s)}{(q(s))^{\frac{1}{3}}}\right) d s=2 \pi\left(\frac{\eta_{0}}{2 \pi \rho_{0} \Omega_{i r w}^{2}}\right)^{\frac{1}{3}} \int_{s_{0}}^{0}\left(\frac{1}{r_{i r w}} \frac{d r_{i r w}}{d s}\right)^{-\frac{1}{3}} d s \approx \\
& 2 \pi\left(\frac{\eta_{0} R_{i r w} \rho_{\kappa}}{2 \pi \rho_{0} \Omega_{i r w}^{2}}\right)^{\frac{1}{3}} \frac{3}{2}\left(L_{2}^{\frac{2}{3}}-\left(L_{2}+s_{0}\right)^{\frac{2}{3}}\right) .
\end{aligned}
$$

Using this approximation, Eq. (3.17) is solved iteratively for $s_{0}$, with $s=0$ and $t_{0}=0$, on the domain $\left[-L_{2}, 0\right]$. The initial layer thickness on $L_{2}$ is defined according to:

$$
H_{2}=H \sqrt[3]{\frac{n_{r} r_{r o l} \hat{f}_{s, r o l}+r_{i r w} \hat{f}_{s, i r w}}{r_{i r w} \hat{f}_{s, i r w}}},
$$

where the body forces and radii are calculated for $s=0$. This results in a continuous layer thickness transition between domain I and II.

\subsubsection{Parameters}

The angular velocity of the inner raceway is chosen to be the reference angular velocity $\Omega=\Omega_{i r w}$. Considering pure rolling, the angular velocity of the cage and the rollers are defined by:

$$
\Omega_{c a}=\frac{\frac{1}{2} r_{i r w}}{R_{r o l}+r_{i r w}} \Omega_{i r w}, \quad \text { with } \quad r_{i r w} \quad \text { at } \quad s=\frac{1}{2} L,
$$

and:

$$
\Omega_{\text {rol }}=\frac{r_{i r w}}{2 R_{\text {rol }}} \Omega_{i r w}+\Omega_{c a}, \quad \text { with } \quad r_{i r w} \quad \text { at } \quad s=\frac{1}{2} L,
$$

respectively. The independent geometry parameters are $L, R_{\text {rol }}, R_{i r w}, \rho_{\kappa}$ and the number of rollers $n_{r}$. The parameters $R_{\text {rol }}, R_{\text {irw }}$ and $\rho_{\kappa}$ are expressed in terms of $B, D_{1}$ and $d_{2}$, see Figure 3.9 and Table 3.1, which are used in tables for standard bearing geometry and can be taken from handbooks and open literature. Additional geometry parameters are calculated using the equations given in Table 3.1 . 


\begin{tabular}{|l|l|l|}
\hline \multicolumn{3}{|c|}{ Independent geometry parameters } \\
\hline$\rho_{\kappa}$ & $=$ & $\frac{1}{2} \sqrt{B^{2}+D_{1}^{2}}$ \\
\hline$R_{\text {rol }}$ & $=$ & $\rho_{\kappa}-\frac{1}{2} \sqrt{\frac{1}{4} B^{2}+\left(\rho_{\kappa}+\frac{1}{2} d_{2}\right)^{2}}$ \\
\hline$R_{\text {irw }}$ & $=$ & $\frac{1}{2} d_{2}$ \\
\hline$L$ & & \\
\hline$n_{r}$ & & \\
\hline \multicolumn{2}{|c|}{ Additional geometry parameters } \\
\hline$\gamma$ & $=$ & $\arccos \left(\frac{1}{2} \frac{\rho_{\kappa}+R_{\text {irw }}}{\rho_{\kappa}-R_{\text {rol }}}\right)$ \\
\hline$R_{\text {crol }}$ & $=$ & $\frac{1}{2}\left(\rho_{\kappa}+R_{i r w}\right)$ \\
\hline$L_{2}$ & $=$ & $\rho_{\kappa} \gamma-\frac{1}{2} L$ \\
\hline$n_{\text {solids }}$ & $=$ & $n_{r}+2$ \\
\hline
\end{tabular}

Table 3.1: Geometry parameters spherical roller bearing.

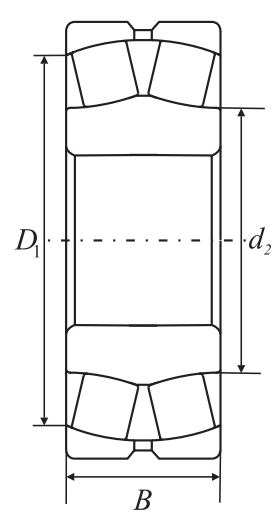

Figure 3.9: Parameters used to define the geometry of a spherical roller bearing.

\begin{tabular}{|c|c|c|c|c|}
\hline \multirow{2}{*}{} & \multicolumn{2}{|c|}{ Range: } & \multicolumn{2}{c|}{ SRB type: } \\
\cline { 2 - 5 } & Min. & Max. & 22205 & 24020 \\
\hline$L / B$ & 0.284 & 0.361 & 0.319 & 0.306 \\
\hline$B / D_{1}$ & 0.198 & 0.493 & 0.407 & 0.379 \\
\hline$d_{2} / D_{1}$ & 0.669 & 0.908 & 0.706 & 0.841 \\
\hline$n_{r}$ & 13 & 40 & 15 & 26 \\
\hline
\end{tabular}

Table 3.2: Typical geometry parameter values for spherical roller bearings, see [2]. 


\subsubsection{Results}

In Figure 3.10 the evolution of the layer thickness as a function of location is shown for a number of moments in time, assuming an initial layer of constant thickness. Also, the characteristic projections are shown. The solution is calculated for the geometry of the spherical roller bearing 22205, see Table 3.2 for the values of the corresponding parameters. The results are presented in terms of the dimensionless time $t / \tau_{c}$, dimensionless layer thickness $\tilde{h} / H$ and dimensionless position $s / L$.

At $s=0$ liquid is flowing into the track. The flow rate at $s=0$ will decrease in time, because the amount of available oil in the reservoir area $L_{2}$ (See Figure 3.8) will decrease in time. This explains the decrease of the layer thickness with time in this region in Figure 3.10. The discontinuity in the slope, appearing in the layer thickness profiles at $t / \tau_{c}=0.3$, $t / \tau_{c}=1$, and $t / \tau_{c}=3$, marks the position of the boundary between the solutions of region I and II. The characteristic projection of this boundary is represented by a dashed line. The layer thickness profiles at $t / \tau_{c}=10$ and $t / \tau_{c}=50$ show the final shape, which will decrease in time at the same rate at every position $s$, according to $\tilde{h} \sim t^{-1 / 2}$. When considering only the flow on the inner raceway of the spherical roller bearing 22205, the layer distribution stays more or less constant, see Figure 2.7 on page 26. Comparing the results of Figure 2.7 and Figure 3.10 shows that the flow on the rollers causes layer enhancement near the outflow side $(s=L)$ and layer reduction near the inflow side $(s=0)$.

In order to study the effect of the geometry parameters on the flow, calculations have been performed for various spherical roller bearings. The range of variation of the geometry parameters, is given in Table 3.2. It is found that, two types of flow behavior can be distinguished. The first type is as shown in Figure 3.10. An example of the second type is shown in Figure 3.11. This solution is calculated for the geometry of the spherical roller bearing 24020, see Table 3.2 for the values of the corresponding parameters. For the second type fluid enters also at $s=L$. The flow is directed towards an attraction point, where a large increase in layer thickness with time is shown. A region III occurs, in which the characteristic projections all originate from $s=L$. It should be mentioned that the real shape and height of the peak in the layer thickness profile cannot be predicted by the present model. The influence of other effects, such as the surface tension and the body force directed normal to the surface, which have been neglected, become significant in this area. Also, the slopes are too large for lubrication theory to hold. In reality such a sharp peak in the layer thickness is not likely to occur. For example, the pressure in the elastohydrodynamic roller-raceway contacts during overrolling will reduce this height.

The appearance of the two types of flow behavior can be explained by considering the $q$ function, Eq. (3.11), and the partial differential equation (3.10). For bearing 22205 the value of $q$ is positive for all values of $s$. For bearing 24020 the $q$ function becomes negative at the right of the peak, which results in a change of direction of the flow. This is shown in Figure 3.12 on page 50. 
Whether the peak occurs or not depends on the values of the geometry parameters. This is shown in Figure 3.13 on page 51. If, for a certain value of $d_{2} / D_{1}$, the value of $B / D_{1}$ is below the curve that corresponds to the number of rollers $n_{r}$, the peak in the layer thickness will occur. The curves have been calculated for $L / B=0.28, L / B=0.32$ and $L / B=0.36$. The results show that the occurrence of the peak is predicted for spherical roller bearings with a large number of rollers, a small bearing width to diameter ratio $B / D_{1}$, a large track width to bearing width ratio $L / B$, and for most cases a large diameter ratio $d_{2} / D_{1}$.
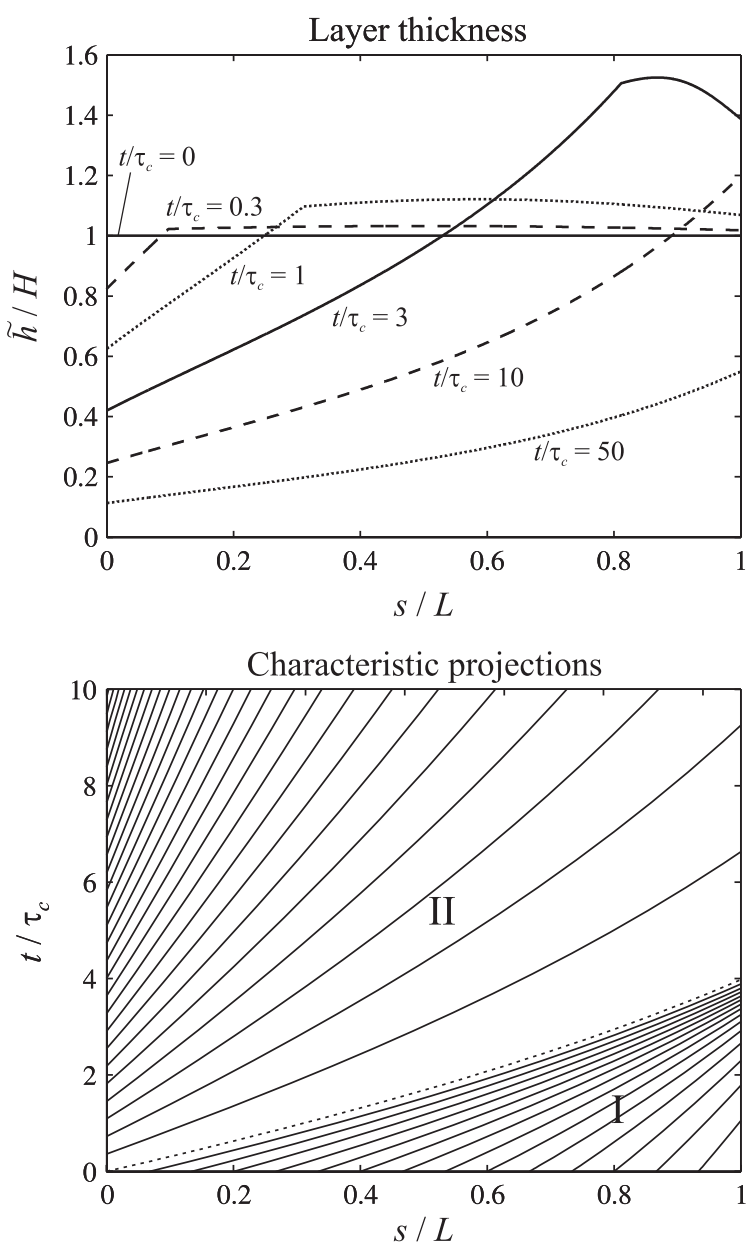

Figure 3.10: SRB 22205 - Top: layer thickness distribution at different times, starting with a uniform layer. Bottom: Characteristic projections. 

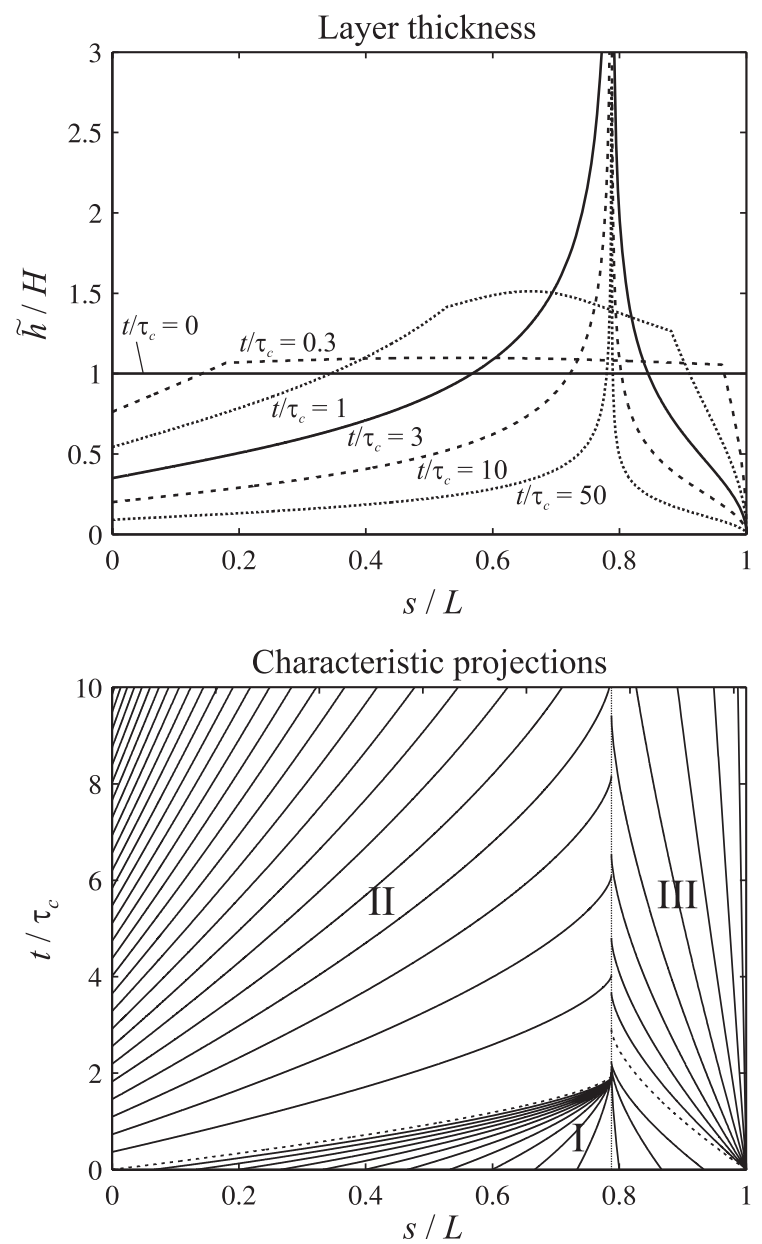

Figure 3.11: SRB 24020 - Top: layer thickness distribution at different times, starting with a uniform layer. Bottom: characteristic projections.
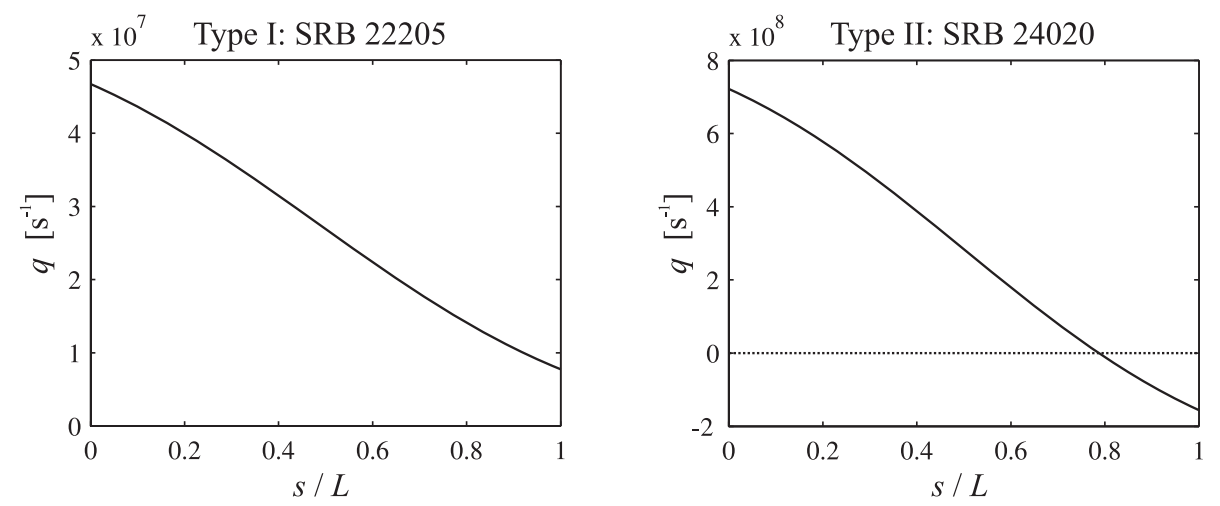

Figure 3.12: $q(s / L)$ for bearing 22205 (flow type I) and for bearing 24020 (flow type II). 

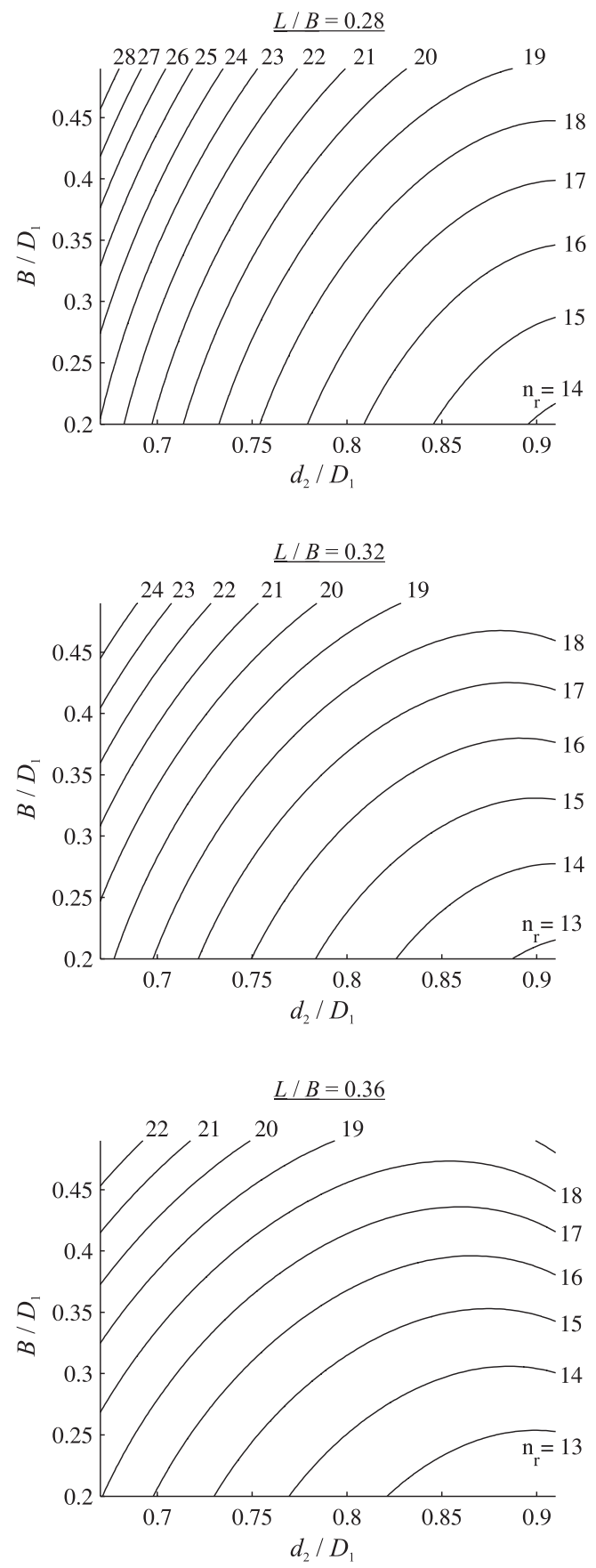

Figure 3.13: Curves for which $q(L)$ is zero. A parameter setting $B / D_{1}$ and $d_{2} / D_{1}$ below the curve $n_{\mathrm{r}}$, denoting the number of rollers, gives a negative value of $q(L)$. 
Next, the layer thickness as a function of the time at the center $s=L / 2$ is studied for various spherical roller bearings. The bearings used in the calculation have various bore diameters: 25, 50, 100 200, 400 and $800 \mathrm{~mm}$. The parameters used are in the range given in Table 3.2. The results are shown in Figure 3.14. The solid lines represent the minimum and maximum values and the dashed line is the mean value. Notice that the solutions form a narrow band. Hence, for various sizes of spherical roller bearings and for different geometry parameter settings, a similar decrease of the layer thickness in the center is predicted.

Finally, as an example of translating these results to "real life", a high speed bearing operating at a rotational speed of $\Omega_{i r w}=10^{3} \mathrm{rad} / \mathrm{s}$ is taken, with $H=10^{-6} \mathrm{~m}$ and $\eta_{0} / \rho_{0}=10^{-5} \mathrm{~m}^{2} / \mathrm{s}$. Substitution into Eq. (2.26) gives a time scale of $\tau_{c}=10 \mathrm{~s}$. Using this time scale value, it can be deduced from Figure 3.14, that the layer thickness reduces by two orders of magnitude in $10^{6} \mathrm{~s} \approx 278 \mathrm{~h}$. Bearings with a large radius generally operate at lower rotational speeds. Hence, the time scale may vary significantly for different bearing sizes. The time scales suggests that for a spherical roller bearing the centrifugal effect may significantly affect the layer thickness distribution within the service life of the bearing. It is emphasized that the actual layer thickness distribution in a bearing application depends on many other phenomena, such as the effect of the flow in the EHL contacts and the supply of lubricant onto the track from the cage. Different physical mechanisms may dominate depending on the operating conditions and bearing type.

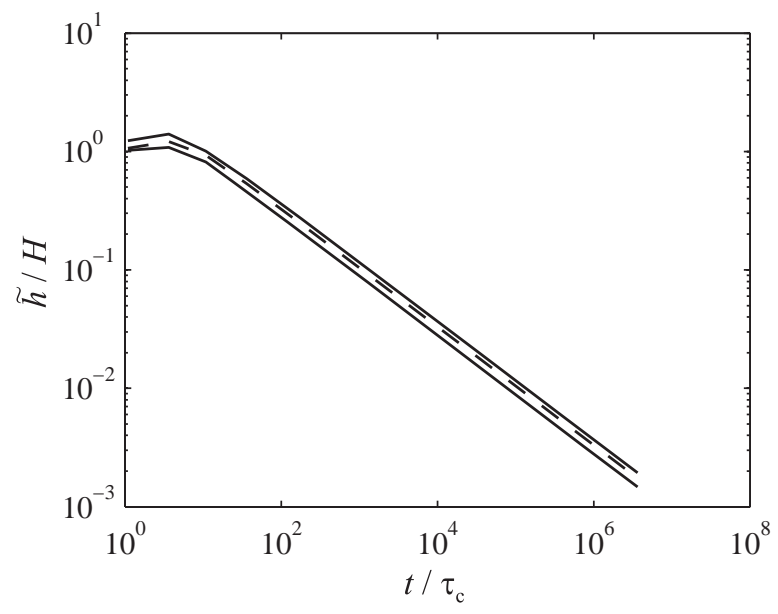

Figure 3.14: Variation of the scaled layer thickness in time at $s=0.5 \mathrm{~L}$ for spherical roller bearings. For parameters, see Table 3.2. 


\subsection{Example 2: tapered roller bearing}

As a second example, the model is applied to a tapered roller bearing. First the equations for the geometry of the rollers and the raceways, the boundary conditions and the independent parameters are specified for this bearing type. Subsequently, the results for the layer thickness in time will be discussed.

\subsubsection{Geometry}

The parameters used to describe the tapered roller bearing are shown in Figure 3.15. The radius function $r(s)$ of the inner raceway, the rollers and the outer raceways are defined as:

$$
\begin{gathered}
r_{i r w}(s)=R_{i r w}+\sin \left(\alpha^{\prime}\right) s, \\
r_{r o l}(s)=R_{r o l}+\sin \left(\gamma-\alpha^{\prime}\right)\left(s-\frac{1}{2} L\right),
\end{gathered}
$$

and:

$$
r_{\text {orw }}(s)=r_{i r w}(s)+2 \cos (\gamma) r_{\text {rol }}(s),
$$

respectively. The axial position on the roller is defined as:

$$
z_{\text {rol }}(s)=\cos \left(\gamma-\alpha^{\prime}\right)\left(s-\frac{1}{2} L\right) .
$$

The total track length $\left(l_{t}\right)$ is defined by Eq. (3.23).
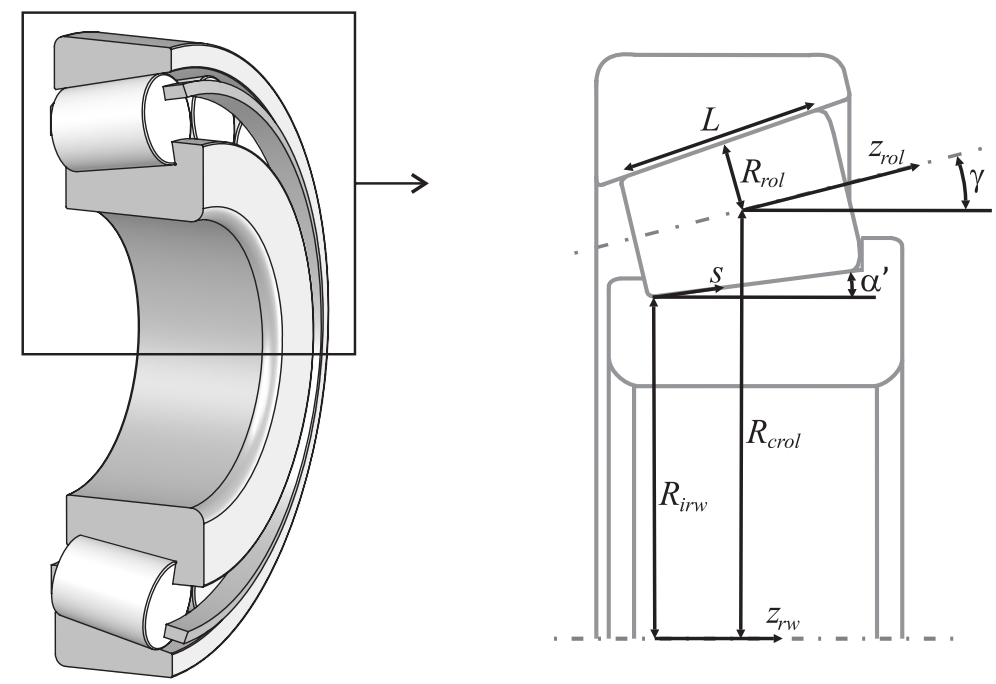

Figure 3.15: Sectional view of a tapered roller bearing including the relevant geometry parameters. 


\subsubsection{Initial and boundary conditions}

In the layer thickness model for the spherical roller bearing lubricant is flowing onto the track at $s=0$. However, for the tapered roller bearing there is a flange blocking the flow at the side of the roller, see Figure 3.15. Therefore, for this bearing type a zero inflow boundary condition is used at $s=0$. A similar boundary condition has been used for the tapered raceway, discussed in section 2.3.1. The starting positions of the characteristics for region I and II are (see Figure 3.7 on page 44):

Region I: $\tilde{h}_{0}=\tilde{h}_{0}^{I}\left(s_{0}\right), t_{0}=0$ and $s_{0}$ is found by solving Eq. (3.17) for a given $s$ and $t$.

Region II: $t_{0}=0, s_{0}=0$ and $\tilde{h}_{0}$ is found by solving Eq. (3.17) for a given $s$ and $t$.

The location of the boundary between domain I and II at time $t$ is found by solving Eq. (3.17) for $s$, with $h_{0}=h_{0}(0), s_{0}=0$ and $t_{0}=0$. In this example the initial layer thickness distribution is uniform $\tilde{h}_{0}^{I}\left(s_{0}\right)=H$.

\subsubsection{Parameters}

The angular velocity of the inner raceway is chosen to be the reference angular velocity $\Omega=\Omega_{\text {irw }}$ and the angular velocities of the cage and the rollers are defined by Eq. (3.30) and Eq. (3.31), respectively. The independent geometry parameters are $L, R_{r o l}, R_{i r w}, \alpha^{\prime}$ and the number of rollers $n_{r}$, see Figure 3.15. The other geometry parameters are calculated using:

$$
\gamma=\operatorname{asin}\left(\frac{R_{r o l} \sin \left(\alpha^{\prime}\right)}{R_{\text {irw }}+\frac{1}{2} L \sin \left(\alpha^{\prime}\right)}\right)+\alpha^{\prime},
$$

and:

$$
R_{\text {crol }}=R_{\text {irw }}+\frac{1}{2} L \sin \left(\alpha^{\prime}\right)+R_{\text {rol }} \cos (\gamma)
$$

\subsubsection{Results}

In Figure 3.16 the evolution of the layer thickness as a function of location and time is shown, assuming a uniform initial layer. Also, the characteristic projections are shown. The results are presented in terms of the dimensionless time $t / \tau_{c}$, dimensionless layer thickness $\tilde{h} / H$ and dimensionless position $s / L$. The solution is calculated for the geometry of the tapered roller bearing 30310. For the values of the corresponding parameters the reader is referred to the caption. This bearing geometry was also used to obtain the results for a single layer on the inner raceway in Chapter 2. The layer thickness distribution shown in Figure 3.16 is very similar to the distribution found for the inner raceway (Figure 2.5 on page 24), although the predicted layer thickness decay rate is approximately half the value found earlier. This means that, when taking into account the effect of the rollers and the 
outer raceway, a smaller layer thickness decay rate is predicted. The discontinuity in the slope appearing in the layer thickness profiles marks the location of the boundary of region I and II. The characteristic projection of this boundary is represented by a dashed line.

In Figure 3.16 the layer thickness at the center $s=L / 2$ as a function of the scaled time is shown for the tapered roller bearing 30310 with a taper angle $\alpha^{\prime}=8.9 \operatorname{deg}(\gamma=11 \mathrm{deg})$. The predicted layer thickness decrease is very similar to the decrease predicted for the spherical roller bearing, see Figure 3.14. Also, in Figure 3.16 the results are shown for $\alpha^{\prime}=20 \operatorname{deg}(\gamma=24 \mathrm{deg})$ and $\alpha^{\prime}=30 \mathrm{deg}(\gamma=36 \mathrm{deg})$. At the same layer thickness larger decay rates are predicted for a larger taper angles $\alpha^{\prime}$. The layer thickness for $\alpha^{\prime}=30 \mathrm{deg}$ at later times is roughly two times smaller than for $\alpha^{\prime}=8.9 \mathrm{deg}$.
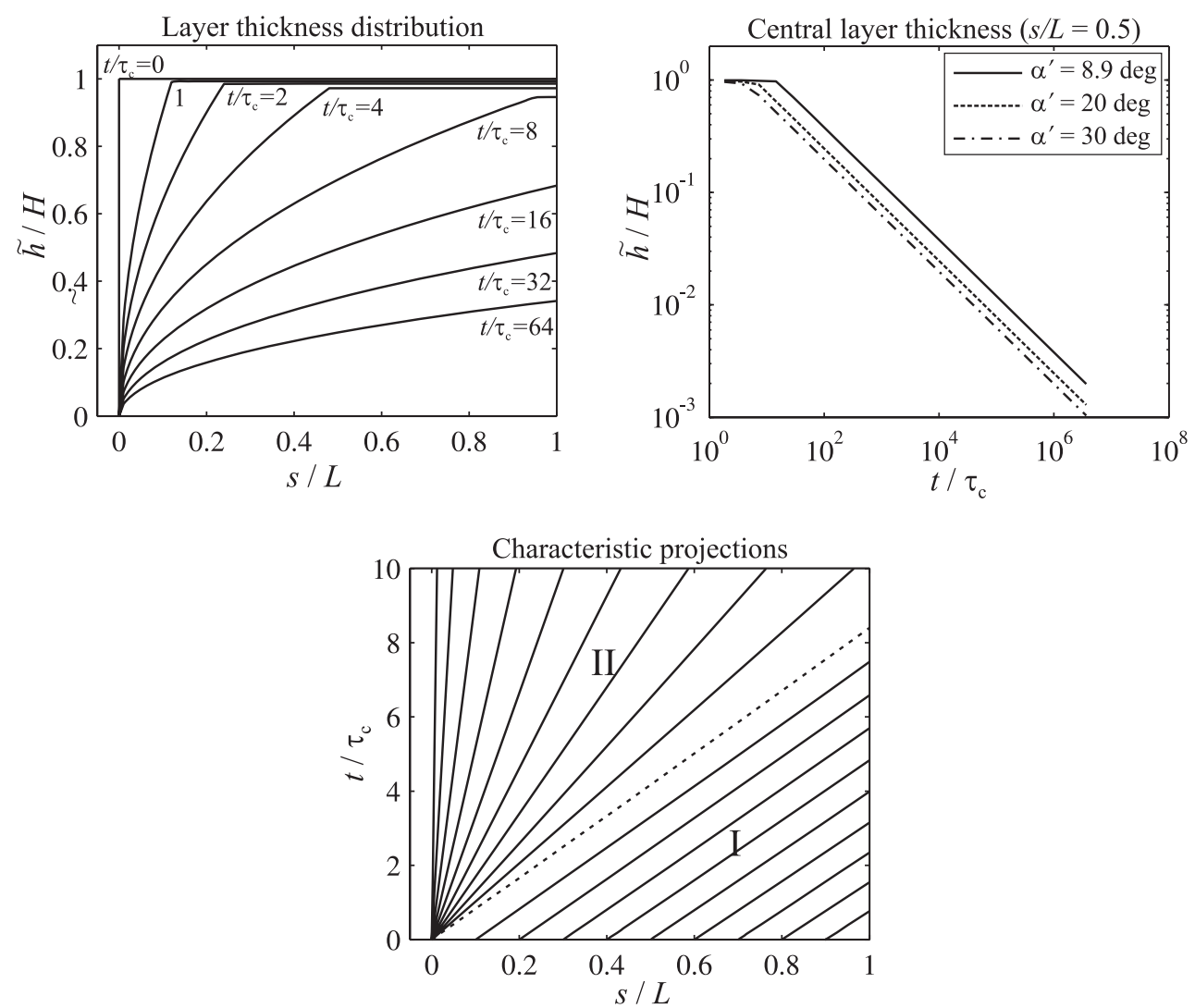

Figure 3.16: Results for tapered roller bearing 30310. Top left: Layer thickness distribution in time, with $\alpha^{\prime}=8.9 \mathrm{deg}$. Top right: Layer thickness in time at $s=\frac{1}{2} L$ for $\alpha^{\prime}=8.9 \mathrm{deg}$, $\alpha^{\prime}=20 \mathrm{deg}$, and $\alpha^{\prime}=30 \mathrm{deg}$. Bottom: Characteristic projections. Other parameters: $R_{\text {rol }}=7.26 \cdot 10^{-3} \mathrm{~m}, R_{\text {irw }}=31.8 \cdot 10^{-3} \mathrm{~m}, L=18.9 \cdot 10^{-3} \mathrm{~m}$, and $n_{r}=16$. 


\subsection{Conclusion}

A physical model and its solution have been presented to predict the change of the lubricant layer thickness distribution on the raceways and the rollers of a roller bearing, due to centrifugal effects. It was shown that the layer thickness can be described by a single firstorder partial differential equation, which is solved using the method of characteristics. It is assumed that the lubricant layer in the EHL contacts equally partitions between the raceway and the roller at the outlet of the contact. This assumption has been verified by experiments, which have shown encouraging results.

It was found that, in addition to the relevant parameters describing the shape of the surfaces on which the lubricant flows and the number of rolling elements, the flow is described by a characteristic time scale parameter. It was shown that, according to the model equations, the change of the layer thickness distribution is independent of the size of the bearing.

As an example, for a spherical roller bearing and a tapered roller bearing, the results for the layer thickness distribution as a function of time were presented. For the spherical roller bearing, two types of characteristic flow behavior may occur. For the first flow type the solution is smooth and at the edge liquid is flowing out of the domain. For the second type a peak in the layer thickness distribution emerges and liquid enters the domain at both sides.

For various spherical roller bearings the central layer thickness as a function of time has been calculated. It was found that the layer thickness decay rates are similar for these bearings. For a typical parameter setting of a high speed bearing it was shown that centrifugal effects significantly affect the layer thickness distribution, within the service life of the bearing. The results for the tapered roller bearing show similar layer thickness decay rates. 


\section{Chapter 4}

\section{ELASTO-HYDRODYNAMIC LUBRICATION}

In addition to the centrifugal force, the flow in the EHL contacts also affects the thickness of the lubricant layers on the track. In the next part of this thesis this effect is studied in detail. This requires solving the EHL contact problem. Therefore, in this chapter the theoretical model and experimental method to analyze single EHL contact behavior are described.

The Hertzian dry contact solution is used for scaling of the EHL equations and it is used as an initial approximation in the numerical solution process. Since the model is thoroughly described in the literature, see for example Moes [51], in this work only an overview of the relevant equations is given.

\subsection{Dry contact model}

Hertz [37] derived an analytical model for concentrated contact between two isotropic, homogeneous, linear elastic solids with smooth surfaces. The undeformed geometry of the solids, denoted by 1 and 2, is defined by the radii of curvature $R_{x, 1}, R_{y, 1}$ and $R_{x, 2}, R_{y, 2}$ in the principle directions, see Figure 4.1. When the solids are pressed together with a force $F$ directed normal to the surfaces, an approximately elliptic or circular contact area is formed.

Undeformed geometry

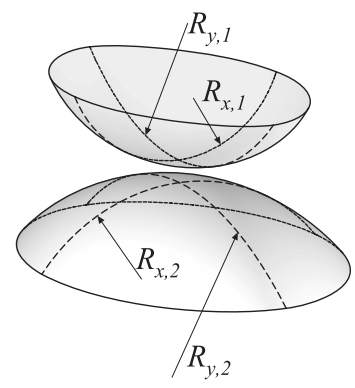

Deformed geometry

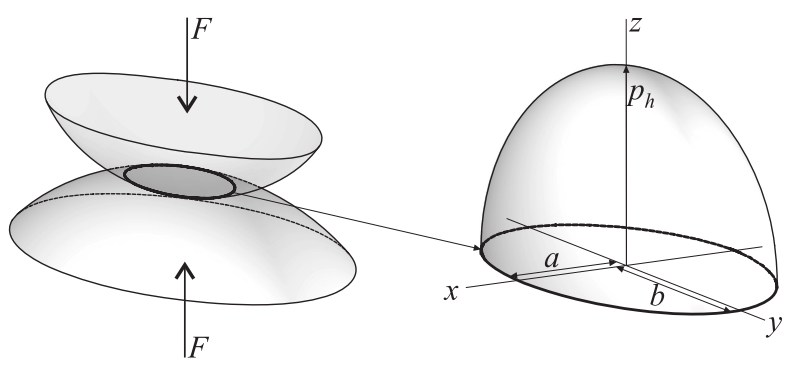

Figure 4.1: Definition of the radii of curvature of the undeformed solid surfaces and Hertzian contact parameters. 
The principle radii of the contact area in $x$ and $y$ direction are defined by:

$$
a=\left(\frac{3 F R}{E^{\prime}}\right)^{\frac{1}{3}}\left(\frac{2 \kappa \mathcal{E}}{\pi}\right)^{\frac{1}{3}}
$$

and:

$$
b=a / \kappa,
$$

respectively, with $0<\kappa \leq 1$.

$R=\left(R_{x}^{-1}+R_{y}^{-1}\right)^{-1}$ is the reduced radius of curvature of the undeformed surface geometry. $R_{x}$ and $R_{y}$ are the reduced radii of curvature in the $x$ and $y$ direction defined, using the radii of curvature of solids 1 and 2, as:

$$
\begin{aligned}
& R_{x}^{-1}=\left(R_{x, 1}^{-1}+R_{x, 2}^{-1}\right)^{-1}, \\
& R_{y}^{-1}=\left(R_{y, 1}^{-1}+R_{y, 2}^{-1}\right)^{-1} .
\end{aligned}
$$

$E^{\prime}$ is the reduced modulus of elasticity:

$$
E^{\prime}=2\left(\frac{1-v_{1}^{2}}{E_{1}}+\frac{1-v_{2}^{2}}{E_{2}}\right)^{-1},
$$

where $E_{1}$ and $E_{2}$ denote the elastic modulus of solids 1 and 2, respectively, and $v_{1}$ and $v_{2}$ the Poisson's ratio of solids 1 and 2 .

The ellipticity ratio $\kappa$ is defined implicitly:

$$
\frac{R_{x}}{R_{y}}=\kappa^{2} \frac{\mathcal{K}-\mathcal{E}}{\mathcal{E}-\kappa^{2} \mathcal{K}},
$$

and can, for a given configuration, easily be solved numerically. $\mathcal{K}$ and $\mathcal{E}$ are the elliptic integrals of the first and second kind defined by:

$$
\mathcal{K}(m)=\int_{0}^{\pi / 2}\left(1-m^{2} \sin ^{2}(\psi)\right)^{-1 / 2} d \psi
$$

and:

$$
\mathcal{E}(m)=\int_{0}^{\pi / 2} \sqrt{1-m^{2} \sin ^{2}(\psi)} d \psi,
$$

respectively, where $m=\sqrt{1-\kappa^{2}}$. Accurate approximations to $\kappa, \mathcal{K}$ and $\mathcal{E}$ as a function of the gap curvature ratio $R_{x} / R_{y}$ are given by Moes [51]. 
The mutual approach of two remote points in the solids is defined by:

$$
c=\frac{a^{2}}{2 R} \frac{\mathcal{K}}{\mathcal{E}} .
$$

The pressure distribution, derived by Hertz, is a semi-ellipsoid as is illustrated in Figure 4.1:

$$
p=p_{h} \sqrt{1-\left(\frac{x}{a}\right)^{2}-\left(\frac{y}{b}\right)^{2}}
$$

where $p_{h}$ is the maximum pressure:

$$
p_{h}=\frac{3 F}{2 \pi a b},
$$

where $a$ and $b$ are given in Eq. (4.1) and Eq. (4.2), respectively.

\subsection{Elasto-Hydrodynamic Lubrication model}

The classical EHL model involves the Reynolds equation, the gap geometry equation, the force balance equation, a viscosity-pressure relation and a density-pressure relation. These equations are discussed in the subsequent sections.

\subsubsection{The Reynolds equation}

The Reynolds equation [58] is the equivalent of the thin layer equation (2.33) for the case of a liquid film in the gap between two moving solid surfaces. Using the Reynolds equation the hydrodynamic pressure that is generated in this film can be calculated. The flow is described using a Cartesian coordinate system $(x, y, z)$, see Figure 4.2.

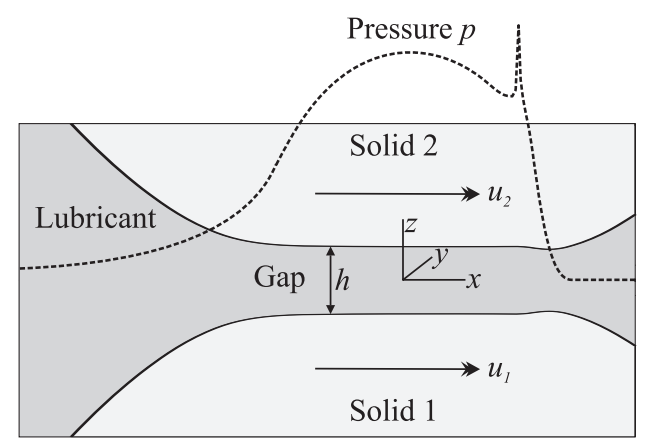

Figure 4.2: Lubricant layer between two deformed moving solids. 
It is assumed that:

- $\quad$ the motion of the two solid surfaces is in the $x$ direction, with a velocity of $u_{1}$ and $u_{2}$, respectively;

- the gap is assumed to be fully filled with liquid. Hence, the gap height equals the liquid film thickness $h=h(x, y, t)$;

- the thickness of the lubricant film is thin compared to the size of the contact area;

- $\quad$ the lubricant film is relatively smooth: $|\nabla h|^{2} \ll 1$;

- $\quad$ the liquid behaves Newtonian;

- $\quad$ the body forces and the inertia terms can be neglected;

- $\quad$ there is no slip between the liquid particles and the solid surfaces;

- the temperature is constant across the gap.

For a detailed derivation of the Reynolds equation the reader is referred to Venner [65]. At the conditions listed above the pressure $p$, the density $\rho$ and the dynamic viscosity $\eta$ are approximately constant over the thickness of the film.

The Reynolds equation for the film thickness $h(x, y, t)$ then reads:

$$
\frac{\partial}{\partial x}\left(\frac{\rho h^{3}}{12 \eta} \frac{\partial p}{\partial x}\right)+\frac{\partial}{\partial y}\left(\frac{\rho h^{3}}{12 \eta} \frac{\partial p}{\partial y}\right)=\frac{\partial \rho h u_{m}}{\partial x}+\frac{\partial \rho h}{\partial t},
$$

where $t$ is the time and $u_{m}$ is the average velocity of the two surfaces, i.e. $u_{m}=\frac{1}{2}\left(u_{1}+u_{2}\right)$. In general for EHL contacts the variation of $u_{m}$ with $x$ can be neglected and for steady state conditions the Reynolds equation reduces to:

$$
\frac{\partial}{\partial x}\left(\frac{\rho h^{3}}{12 \eta} \frac{\partial p}{\partial x}\right)+\frac{\partial}{\partial y}\left(\frac{\rho h^{3}}{12 \eta} \frac{\partial p}{\partial y}\right)=u_{m} \frac{\partial \rho h}{\partial x} .
$$

In Eq. (4.12) $p$ is the pressure relative to the atmospheric pressure. Only pressures larger than the vapor pressure are allowed in the liquid film. The vapor pressure is generally very small compared to the contact pressures and it is therefore justified to approximate the vapor pressure by the atmospheric pressure $p=0$. Hence, in the EHL model only positive pressures relative to the atmospheric pressure $p \geq 0$ should occur.

\subsubsection{Gap height equation}

The height of the gap between the two solids is determined by the geometry of the undeformed solid surfaces, the mutual distance of approach $\delta$ of the solids, and the elastic deformation of the solids due to the contact pressure in the lubricant film. The dimension of the contact is small compared to the radii of curvature of the undeformed solids, and therefore the undeformed geometry can be approximated by paraboloid surfaces. 
The equation for the gap height reads:

$$
h(x, y)=\overbrace{-\delta}^{\substack{\text { mutual } \\
\text { approach }}}+\frac{\begin{array}{c}
\text { undeformed } \\
\text { geometry }
\end{array}}{2 R_{x}}+\frac{y^{2}}{2 R_{y}}+\overbrace{\frac{2}{\pi E^{\prime}} \iint_{A} \frac{p\left(x^{\prime}, y^{\prime}\right)}{\sqrt{\left(x-x^{\prime}\right)^{2}+\left(y-y^{\prime}\right)^{2}}} d x^{\prime} d y^{\prime}}^{\begin{array}{c}
\text { elastic } \\
\text { deformation }
\end{array}}
$$

where $A$ is the contact area in the $(x, y)$-plane. The elastic deformation is obtained by considering each solid as a semi-infinite elastic solid. For a detailed derivation the reader is referred to Johnson [41].

\subsubsection{Force balance equation}

For steady state conditions, the load that acts normal to the contact, i.e. $F$, is balanced by the pressure generated in the film:

$$
F=\iint_{A} p(x, y) d x d y
$$

where $A$ is the contact area in the $(x, y)$-plane. This condition determines the value of the mutual approach of the solids $\delta$ in Eq. (4.13).

\subsubsection{Lubricant viscosity and density}

Due to the high pressures that commonly occur in EHL contacts, in the contact the viscosity of the lubricant can vary many orders of magnitude. Ertel [30] and Grubin [34] were the first to include this effect in a theoretical model. They showed that due to the increased viscosity in the contact a significantly thicker film occurs. In their analysis they used the exponential viscosity-pressure relation ascribed to Barus [4] in 1891:

$$
\eta(p)=\eta_{0} e^{\alpha p}
$$

where $\alpha$ is the pressure-viscosity coefficient and $\eta_{0}$ the dynamic viscosity at ambient pressure. This relation is accurate for relative low pressures only. In the present study, the empirical relation presented by Roelands [59] is used, which describes the viscosity for pressures up to $1 \mathrm{GPa}$ :

$$
\eta(p)=\eta_{0} \exp \left\{\left(\frac{\alpha p_{r}}{z_{R}}\right)\left(-1+\left(1+\frac{p}{p_{r}}\right)^{z_{R}}\right)\right\},
$$

where $p_{r}=1.96 \cdot 10^{8} \mathrm{~Pa}$ and:

$$
z_{R}=\frac{p_{r} \alpha}{\ln \left(\eta_{0} / \eta_{\text {dim }}\right)+9.67}
$$

where $\eta_{\mathrm{dim}}=1 \mathrm{~Pa} \cdot \mathrm{s}$. 
Also, due to the high pressures the density variation of the lubricant is significant. The density-pressure relation is modeled using the empirical relation presented by Dowson and Higginson [28]:

$$
\rho(p)=\rho_{0} \frac{0.59 \cdot 10^{9}+1.34 p}{0.59 \cdot 10^{9}+p} .
$$

In the classical EHL model the viscosity-pressure relation and density-pressure relation are used as input to the Reynolds equation. Strictly, this is not allowed as the Reynolds equation is obtained based on the assumption that the viscosity is constant. Rajagopal and Szeri [57] showed that a consistent derivation of the equations of EHL, that takes into account the pressure dependence of viscosity, leads to additional terms in the governing equations. They showed that the viscosity may differ significantly in the high pressure region. Schäfer et al. [60] concluded that in the case of sliding contacts these extra terms can be significant, but for pure rolling it is permissible to neglect these additional terms and use the Reynolds equation.

\subsection{Dimensionless equations}

The equations are made dimensionless using the following dimensionless parameters:

$$
\begin{array}{ll}
X=x / a, & Y=y / b, \\
H=h / c, & \Delta=\delta / c, \\
P=p / p_{h}, & \bar{\eta}=\eta / \eta_{0}, \\
\bar{\rho}=\rho / \rho_{0}, & \bar{\alpha}=\alpha p_{h} .
\end{array}
$$

Substitution of Eq. (4.19) and Eq. (4.2) into Eq. (4.12) gives:

$$
\frac{\partial}{\partial X}\left(\frac{\bar{\rho} H^{3}}{\bar{\eta} \lambda} \frac{\partial P}{\partial X}\right)+\kappa^{2} \frac{\partial}{\partial Y}\left(\frac{\bar{\rho} H^{3}}{\bar{\eta} \lambda} \frac{\partial P}{\partial Y}\right)=\frac{\partial(\bar{\rho} H)}{\partial X},
$$

with:

$$
\lambda=\frac{12 u_{m} \eta_{0} a}{c^{2} p_{h}}
$$

The gap height equation (4.13) in dimensionless variables reads:

$$
H(X, Y)=-\Delta+\mathcal{S} X^{2}+(1-\mathcal{S}) Y^{2}+\frac{1}{\pi \mathcal{K}} \iint_{A} \frac{P\left(X^{\prime}, Y^{\prime}\right)}{\sqrt{\kappa^{2}\left(X-X^{\prime}\right)^{2}+\left(Y-Y^{\prime}\right)^{2}}} d X^{\prime} d Y^{\prime},
$$

where $\mathcal{S}$ is the shape parameter defined by:

$$
\mathcal{S}=\frac{\mathcal{E}-\kappa^{2} \mathcal{K}}{\mathcal{K}-\kappa^{2} \mathcal{K}} .
$$


The dimensionless force balance equation reads:

$$
\frac{2 \pi}{3}=\iint_{A} P(X, Y) d X d Y,
$$

where $A$ is the contact area in the $(X, Y)$-plane. The dimensionless density-pressure relation is defined by:

$$
\bar{\rho}(P)=\frac{0.59 \cdot 10^{9}+1.34 p_{h} P}{0.59 \cdot 10^{9}+p_{h} P} .
$$

The Barus viscosity-pressure relation in dimensionless variables is:

$$
\bar{\eta}(P)=e^{\bar{\alpha} P}
$$

and the dimensionless Roelands viscosity-pressure relation reads:

$$
\bar{\eta}(P)=\exp \left\{\left(\frac{\alpha p_{r}}{z_{R}}\right)\left(-1+\left(1+\frac{p_{h}}{p_{r}} P\right)^{z_{R}}\right)\right\} .
$$

\subsection{Parameters}

For an incompressible liquid obeying the Barus viscosity-pressure relation (4.26) the problem is fully defined by three non-dimensional parameters, for example: $\lambda, \kappa$ and $\bar{\alpha}$. Also other parameters can be chosen. Frequently used parameter groups for circular or elliptical contacts have been derived by Moes [51]:

$$
\begin{gathered}
M=\frac{F}{E^{\prime} R_{x}^{2}}\left(\frac{E^{\prime} R_{x}}{2 u_{m} \eta_{0}}\right)^{\frac{3}{4}}, \\
L=\alpha E^{\prime}\left(\frac{2 u_{m} \eta_{0}}{E^{\prime} R_{x}}\right)^{\frac{1}{4}},
\end{gathered}
$$

and:

$$
D=\frac{R_{x}}{R_{y}} .
$$

For elliptical contacts Moes also defined $N=M \sqrt{D}$, which can be used instead of $M$. The relation between the parameters $\lambda$ and $\bar{\alpha}$ and Moes' parameters is defined according to:

$$
\lambda=\pi\left(\frac{128}{3 M^{4}} \frac{16 \pi \mathcal{E}^{5}}{\kappa^{4}(1+D)^{5} \mathcal{K}^{6}}\right)^{\frac{1}{3}}
$$


and:

$$
\bar{\alpha}=\frac{L}{\pi}\left(\frac{3 M \pi^{2} \kappa}{2} \frac{(1+D)^{2}}{16 \mathcal{E}^{2}}\right)^{\frac{1}{3}}
$$

When the Roelands viscosity-pressure relation (4.27) and the density-pressure relation (4.25) are used, two additional parameters are introduced in the model, for example the pressure-viscosity coefficient $\alpha$ and the viscosity at ambient viscosity $\eta_{0}$.

\subsection{Starved lubrication model}

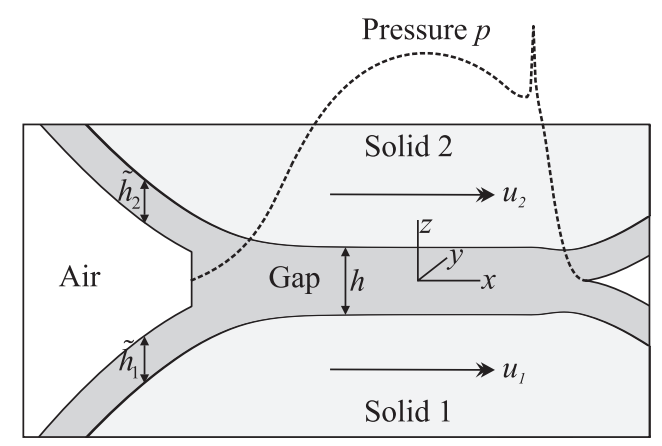

Figure 4.3: Starved lubrication.

When the contact is starved, the pressure build-up starts at a small distance in front of the contact, see Figure 4.3. Compared to fully flooded lubrication, shown in Figure 4.2, this situation is more complex, as the boundary of the pressurized region is now a free boundary. Its location is determined by the shape and thickness of the lubricant layers on the running surfaces in the inlet to the contact. The total thickness of these layers is defined by $h_{\text {oil }}=\tilde{h}_{1}+\tilde{h}_{2}$. In the starved model an additional variable is introduced, the so-called fractional film content, see Elrod [29], representing the ratio $h_{\text {oil }}$ and the actual gap height $(h)$ :

$$
\theta=h_{\text {oil }} / h=H_{\text {oil }} / H .
$$

Inside the pressurized region, the lubricant completely fills the gap between the solid surfaces and in the starved/cavitated regions $\theta$ in unknown and needs to be solved. The following complementarity condition must be fulfilled:

$$
P(X, Y)(\theta(X, Y)-1)=0,
$$

with $P(X, Y) \geq 0$ and $0 \leq \theta(X, Y)<1$. Bayada [5] included the fractional film content into the Reynolds equation (4.20). This modified the Reynolds equation, which in dimensionless form reads: 


$$
\frac{\partial}{\partial X}\left(\frac{\bar{\rho} H^{3}}{\bar{\eta} \lambda} \frac{\partial P}{\partial X}\right)+\kappa^{2} \frac{\partial}{\partial Y}\left(\frac{\bar{\rho} H^{3}}{\bar{\eta} \lambda} \frac{\partial P}{\partial Y}\right)-\frac{\partial(\theta \bar{\rho} H)}{\partial X}=0,
$$

which is an equation for the pressure $P$ in the pressurized region and for the fractional film content $\theta$ in the starved/cavitated regions. Equation (4.35) is a hyperbolic equation for $\theta$, so at the inlet of the domain a boundary condition needs to be specified. This boundary condition for $\theta$ is obtained by prescribing the total thickness of the supply layers $H_{\text {oil }}(Y, \bar{t})$ at the inlet of the domain and substituting this into Eq. (4.33).

\subsection{Numerical solution}

The first numerical solution of the EHL problem was presented by Petrusevich in 1951 [56] for line contacts. Based on an extensive series of numerical calculations, accurate film thickness prediction formulas have been obtained for fully flooded EHL line contacts by Dowson and Higginson in 1959 [27] and for fully flooded EHL elliptical contacts by Hamrock and Dowson in 1976 [35]. These formulas are still widely used today. Later the stability and efficiency of the numerical solvers was drastically increased by the introduction of multigrid techniques in EHL by Lubrecht [48], followed by Venner [65]. This allowed accurate and detailed numerical simulations of EHL contact problems, including the effect of time varying conditions, effects of surface waviness/roughness moving through the contact, and effects of starved lubrication [17, 66, 67, 71]. A similar multigrid EHL solver is used in the research presented in this thesis. The dimensionless system of equations is discretized with second-order accuracy on a uniform grid. The essential elements of the method are described in detail in Venner and Lubrecht [67]. The specific details regarding the starved lubrication problem are given by Wijnant [71], Chevalier [16], and Damiens [19].

\subsection{Characteristic theoretical results}

As an example, in Figure 4.4 on page 67 typical results are shown of the dimensionless gap height $H$ and the dimensionless pressure distribution $P$ for a circular EHL contact operating under fully flooded conditions. The parameters values for this case are given in the caption. The liquid enters the domain at $X=-2.5$. In the center of the pressure region the pressure distribution still resembles the semi-elliptical Hertzian dry contact profile. However, at the boundaries of the Hertzian region the pressure variation is smooth, rather than abruptly reaching $P=0$. Furthermore, in the outlet region locally a pressure maximum occurs, known as the Petrusevich pressure spike [56]. In the center of the Hertzian contact region the film thickness is nearly uniform. This is due to the large viscosity as a result of which the terms at the left hand side of the Reynolds equation (4.20) are negligible. In the outlet and to the sides the film thickness decreases which leads to the typical horse shoe shape of the film thickness map. The minimum film thickness occurs in the side lobes.

In Table 4.1 results are given for the central film thickness $H_{\text {cff }}$ (at $X=Y=0$ ) for fully flooded conditions and different grid densities. The 4th column of Table 4.1 shows that the difference between the solutions decreases by a factor 4 when the mesh size is halved. Thus, the solution converges with second order accuracy. As a second order discretization 
is used this shows that the truncation error has converged below the discretization error. The discretization error on level 6 is 4 times smaller than the difference between the values obtained on grid level 5 and 6 . In this example this difference is $0.03 \%$ of the central film thickness.

Next, consider the starved lubricated contact. The results for the dimensionless pressure $P$ and the lubricant distribution $\theta H$ are presented in the bottom part of Figure 4.4. The same parameters have been used as for the fully flooded case. In the pressurized region the product $\theta H$ is the film thickness and outside the pressurized region it is the total amount of lubricant in the gap represented as a single layer. It can be seen as the footprint of the contact, when rolling on a layer of oil equal to all lubricant in the gap situated on the bottom surface. The liquid enters the domain at $X=-2.5$ with a prescribed layer thickness: $\theta H=H_{\text {oil }}$. In this example $H_{\text {oil }}$ is uniform and equal to half the central film thickness for the fully flooded case: $H_{\text {oil }}=0.5 H_{\text {cff }}=0.21$. The results show that a jump in the lubricant distribution $\theta \mathrm{H}$ occurs, just in front of the Hertzian region. At this location the amount of lubricant supplied to the contact is sufficient to fill the gap and the pressure starts to build up. The film thickness is smaller than for the fully flooded case. Also, the pressure distribution more closely resembles the Hertzian pressure distribution and the height of the pressure spike has reduced considerably.

For different grid densities the central film thickness for the starved case $H_{c s}$ is given in Table 4.1. The convergence factor is given in the last column. On contrary to the fully flooded case, it does not show second order behavior. This can be explained by the influence of the boundary of the pressure region on the solution. This boundary is approximately circular and when it is discretized on a Cartesian grid an extra error is introduced. This error is not of second order, see Bos [7]. Furthermore, as the boundary can more accurately be represented on denser grids, its position may change on a denser grid. A change of the position of the boundary has a significant influence on the central film thickness. Therefore, the convergence factor does not always converge to a constant.

\begin{tabular}{|c|c|c|c|c|c|}
\hline \multirow[b]{2}{*}{$\begin{array}{c}\text { Level } \\
(L)\end{array}$} & \multirow[b]{2}{*}{ Mesh } & \multicolumn{2}{|c|}{ Fully flooded } & \multicolumn{2}{|c|}{ Starved } \\
\hline & & $H_{c f f}$ & $\frac{H_{c f f}^{L-1}-H_{c f f}^{L-2}}{H_{c f f}^{L}-H_{c f f}^{L-1}}$ & $H_{c s}$ & $\frac{H_{c s}^{L-1}-H_{c s}^{L-2}}{H_{c s}^{L}-H_{c s}^{L-1}}$ \\
\hline 1 & $65^{2}$ & 0.373824 & & 0.175881340 & \\
\hline 2 & $129^{2}$ & 0.408928 & & 0.175959007 & \\
\hline 3 & $257^{2}$ & 0.417974 & 3.9 & 0.175984527 & 3.0 \\
\hline 4 & $513^{2}$ & 0.420252 & 4.0 & 0.175991897 & 3.5 \\
\hline 5 & $1025^{2}$ & 0.420823 & 4.0 & 0.175993340 & 5.1 \\
\hline 6 & $2049^{2}$ & 0.420965 & 4.0 & 0.175993470 & 11.1 \\
\hline
\end{tabular}

Table 4.1: Dimensionless central film thickness and convergence factors for fully flooded conditions and for starved conditions. Parameters: $M=20, L=10, D=1, \eta_{0}=0.8$ Pa.s , $\alpha=20 \cdot 10^{-9} \mathrm{~Pa}^{-1}$, and $H_{\text {oil }}=\frac{1}{2} H_{\text {cff }}$. 
Fully flooded EHL: liquid completely fills the gap
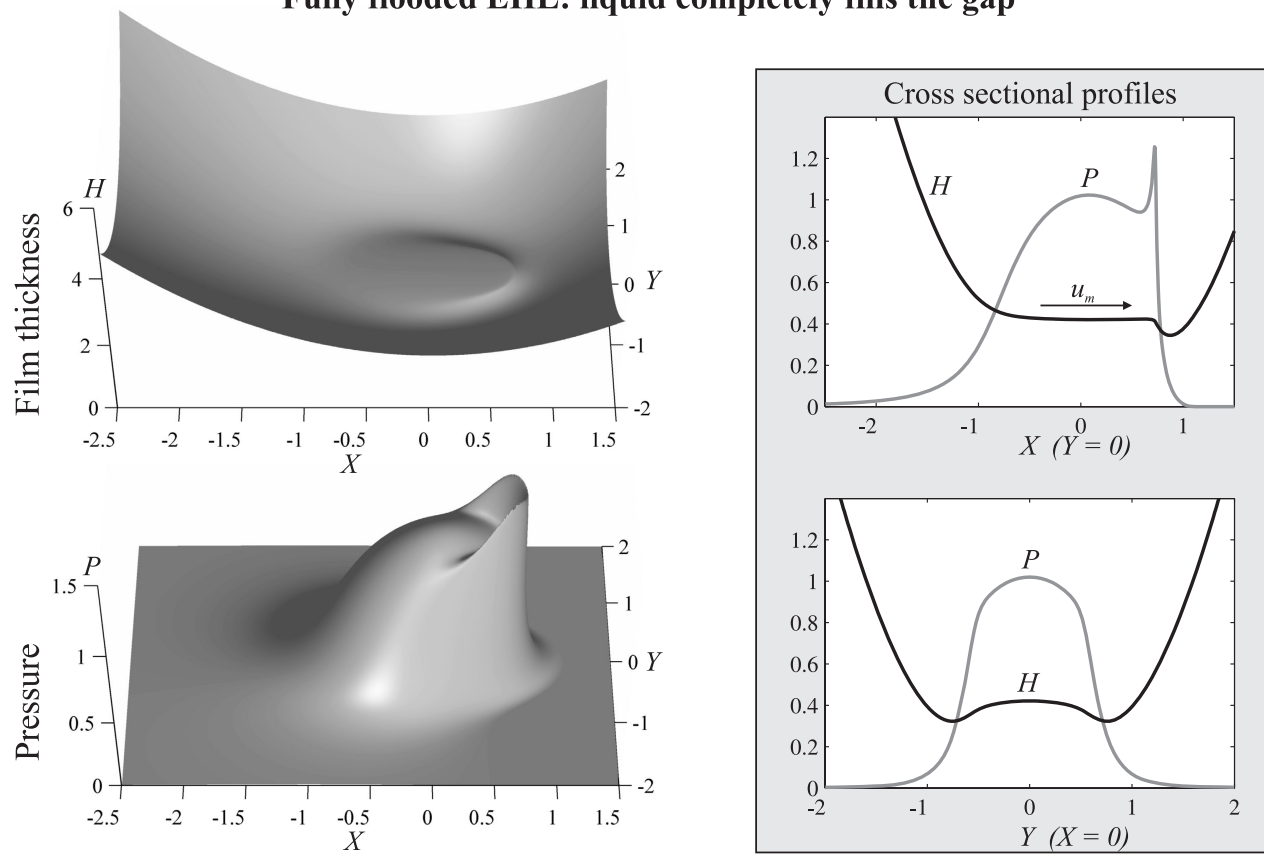

Starved EHL: limited supply of liquid
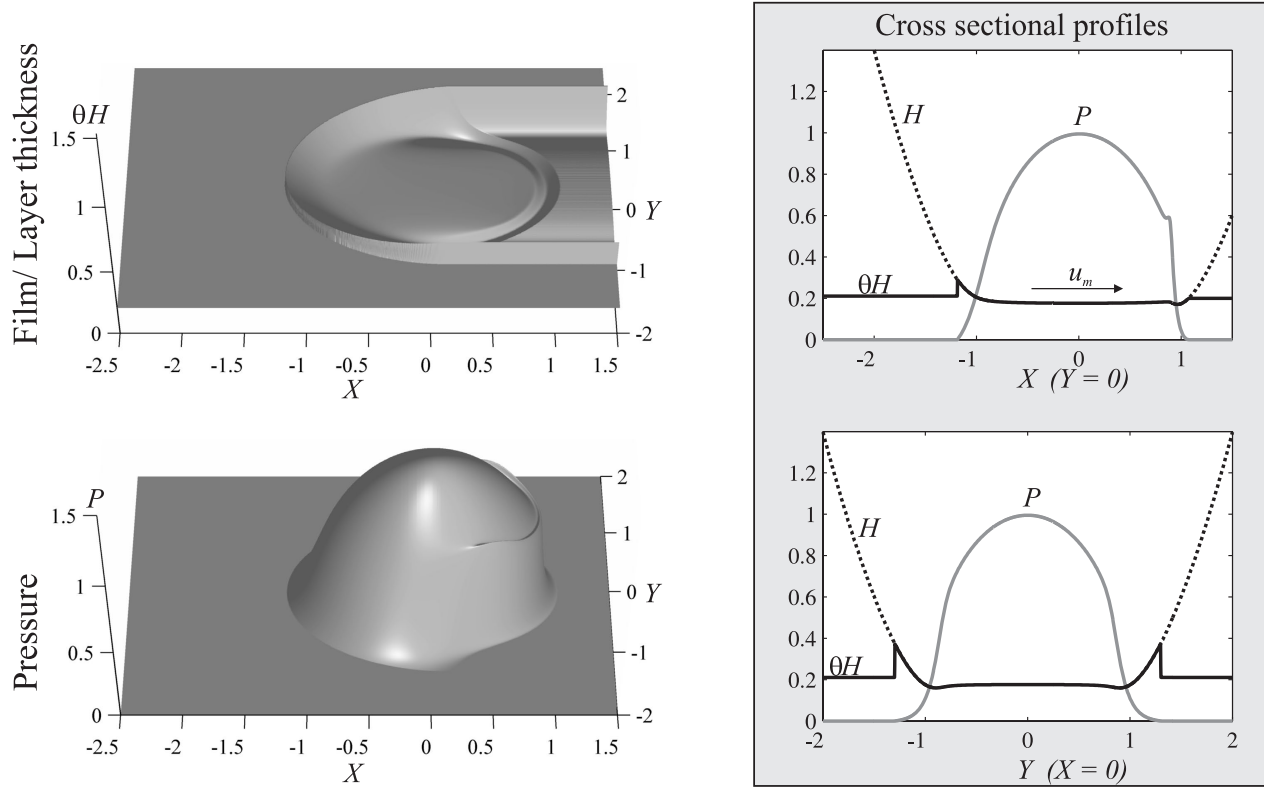

Figure 4.4: Fully flooded and starved EHL. Solution for film thickness $H$, lubricant distribution $\theta H$ and pressure distribution $P$. Parameters: $M=20, L=10, \quad D=1, \quad \eta_{0}=0.8$ Pa.s, $\alpha=20 \cdot 10^{-9} \mathrm{~Pa}^{-1}$, and $H_{\text {oil }}=\frac{1}{2} H_{\text {cff }}$. 


\subsection{Experimental setup}

By using optical interferometry the film thickness in EHL contacts can be measured. Optical interferometry, introduced in lubrication by Gohar and Cameron [33], has been developed to a very high level, allowing measurements of film thicknesses down to just a couple of nanometers. The EHL film thickness measurements presented in this thesis have been performed on an optical EHL device developed at Imperial College, London, UK, based on the research of Foord et al. [31], Cameron and Gohar [8], Wedeven et al. [69], Johnston et al. [42], Cann et al. [14], and others. With this device the film thickness can be measured in the EHL contact between a ball or a roller and a rotating glass disk. In Figure 4.5 a photograph and a sketch of the glass disk and the ball are shown. The disk is supported by a shaft that is driven by an electromotor. The ball is supported by three small bearings and is driven by the disk under pure rolling conditions. To prevent spin of the ball the rotational axis of the ball is directed towards the surface at the center of the disk. The rotational speed of the disk, the load that is applied on the rolling element, and the distance of the contact with respect to the center of the disk can be adjusted.

With an optical system, light from a halogen source is focused on the contact area. The light is partly reflected by a semi-reflective chromium layer at the bottom of the disk. The reflected light interferes with the light reflected at the surface of the ball. The resulting interference patterns are analyzed using a spectrometer that is connected to a computer. Because a silica spacer layer is used between the semi-reflective chromium layer and the oil film, the film thickness can be measured in the range between 1 and $1000 \mathrm{~nm}$ with an accuracy of $\pm 1 \mathrm{~nm}$. By using a color camera and image analysis methods, film thickness maps of the contact region can be obtained. For an extensive description of the experimental method the reader is referred to the articles cited above.
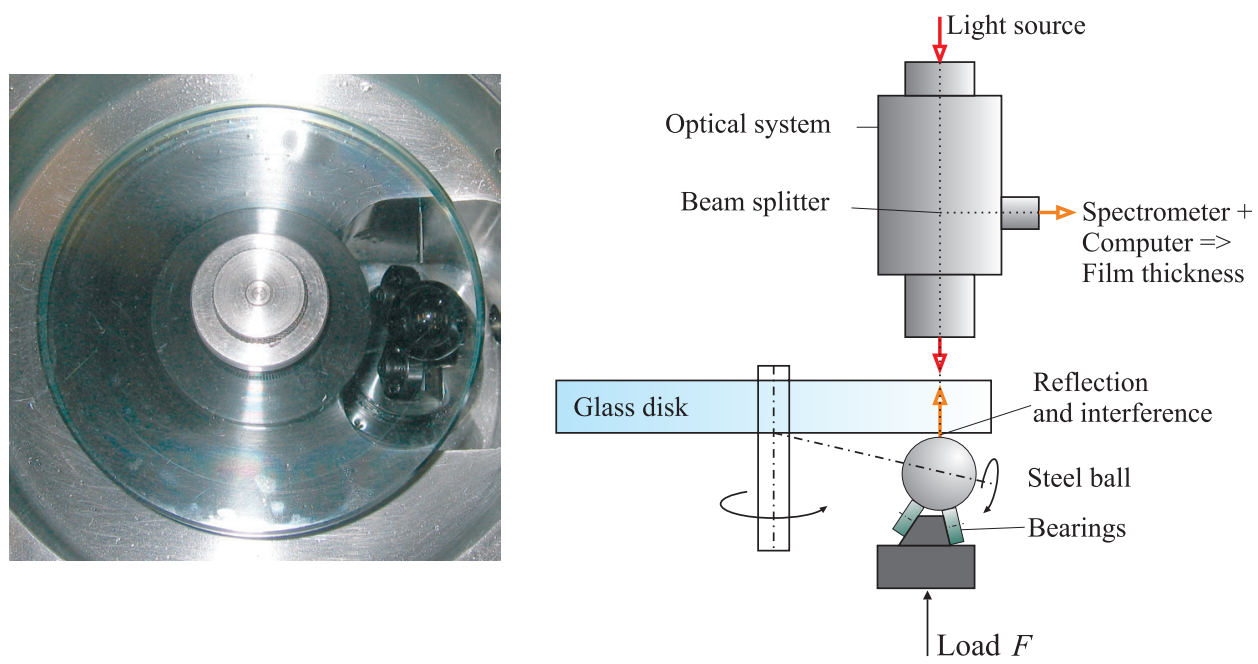

Figure 4.5: Typical setup for optical film thickness measurements for the EHL contact between a glass disk and a steel ball. 


\subsection{Experimental results}

\subsubsection{Fully flooded EHL}

By using the setup described in the previous section, the fully flooded EHL film thickness at the contact center has been measured for different surface velocities $u_{m}$ and oil temperatures. The results are shown in Figure 4.6. The parameters values are given in the caption. Also shown in the figure are the predictions obtained by the Hamrock and Dowson film thickness formula [35] and the results of the present numerical simulations. For this oil the agreement between the measured and the predicted values is very good, even at extremely thin films. The measurement is repeated and a video camera is used to record the optical interference patterns of the contact area. The obtained interferometric images for five velocities are also shown. In these images the inlet of the contact is positioned at the bottom. At high speeds the images clearly show the horseshoe shaped film thickness distribution, as was shown in the theoretical results. At lower speeds the side lobes decrease and the distribution is nearly uniform. Also, at these low film thickness levels some surface roughness effects are visible.

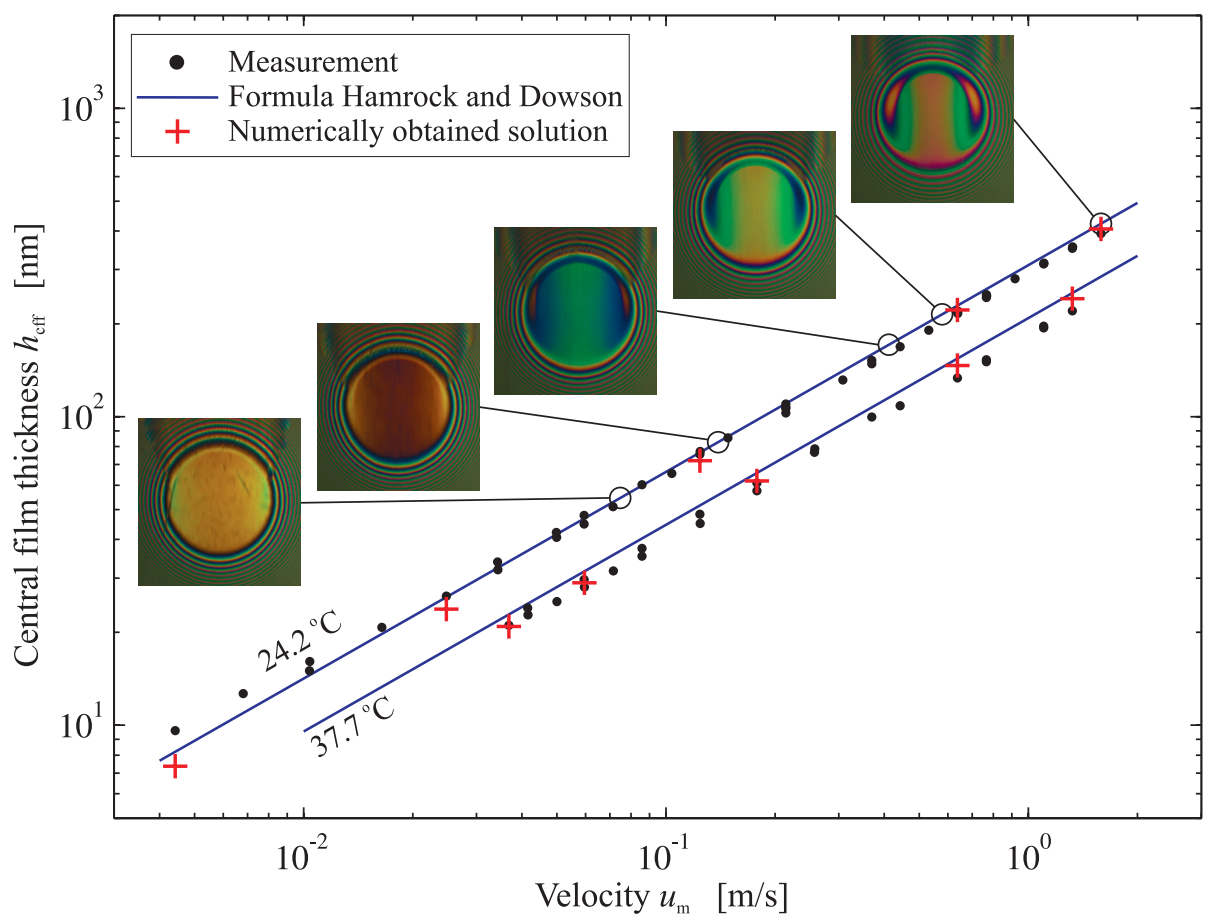

Figure 4.6: Central EHL film thickness as function of velocity $u_{m}$ operating under fully flooded conditions. For five speeds interferometric images are shown. The contact is lubricated with HVI60. Viscosity: $\eta_{0}=0.050$ Pa.s at $24.2^{\circ} \mathrm{C}$ and $\eta_{0}=0.027$ Pa.s at $37.7^{\circ} \mathrm{C}$. Other parameters: load $F=20 \mathrm{~N}$, ball radius $R_{x}=R_{y}=9.525 \mathrm{~mm}$, reduced elastic modulus $E^{\prime}=111.2 \mathrm{GPa}$, and the pressure-viscosity index $\alpha=19.8 \mathrm{GPa}^{-1}$. 


\subsubsection{Starved EHL}

In Figure 4.7 an interferometric image is shown of an EHL contact operating under starved conditions. At the inlet of the contact the track, formed on the disk and the ball at the previous overrollings, is clearly visible. Moreover, a butterfly shaped meniscus is shown. Outside this meniscus the oil partially fills the gap between the ball and the disk.

Due to the pressure distribution in the EHL contact oil flows to the side of the track. When the rate of this side flow in the contact is equal to the rate at which oil flows onto the track from the side, the layer thickness in the track is steady. By means of film thickness measurements using a ball and disk apparatus, Cann et al. [9] showed that the steady state film thickness can be quantified by a single dimensionless parameter consisting of the surface velocity, the contact size, the viscosity, the surface tension and the volume of oil near the track. A relatively small steady state film thickness occurs for large contacts operating at a high speed that are lubricated with a small amount of oil with a high viscosity.

When the side flow out of the contact exceeds the flow from the side onto the track, the layer thickness in the track will gradually decrease. As the film thickness inside the contact is determined by the thickness of the layers in the track, the film thickness will also decrease in time. An example of this transient behavior is shown in Figure 4.8, where the dots represent the measured central film thickness as a function of time. In the next chapter, starved lubricated contacts are studied in more detail. A model will be presented for the prediction of the transient film thickness decay when the reflow of oil onto the track is negligible. The drawn line in Figure 4.8 represents the predictions of this model. 


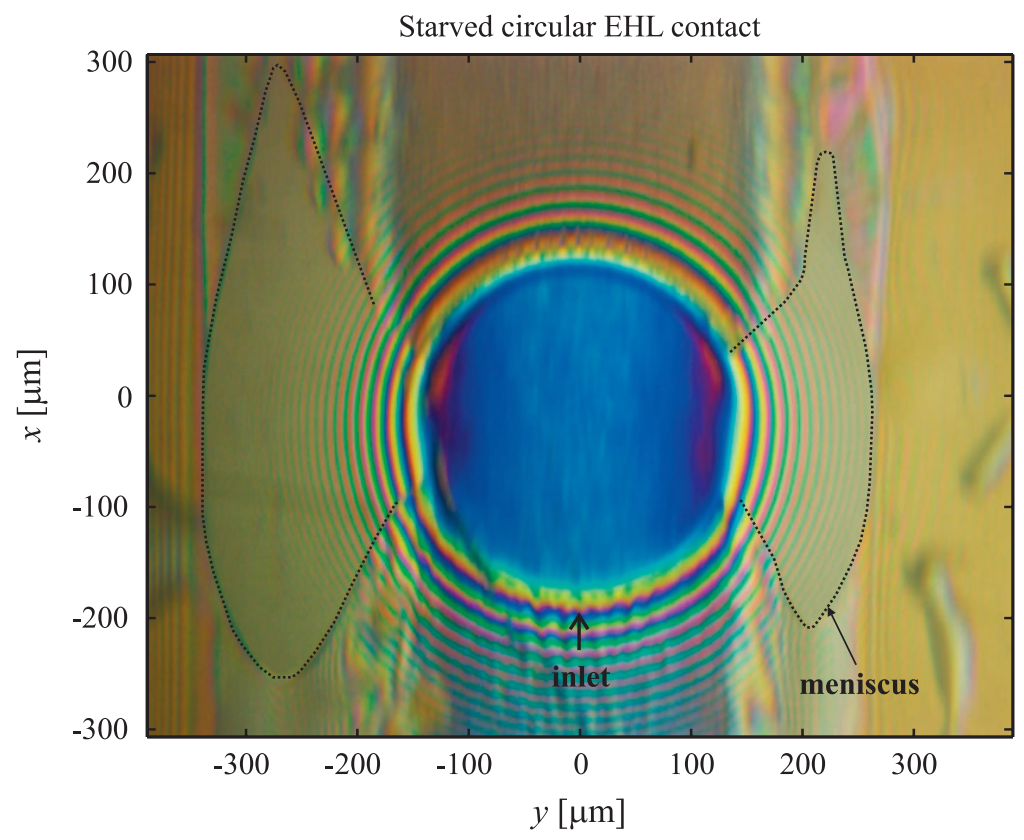

Figure 4.7: Interferometric image of an EHL contact operating under starved conditions.

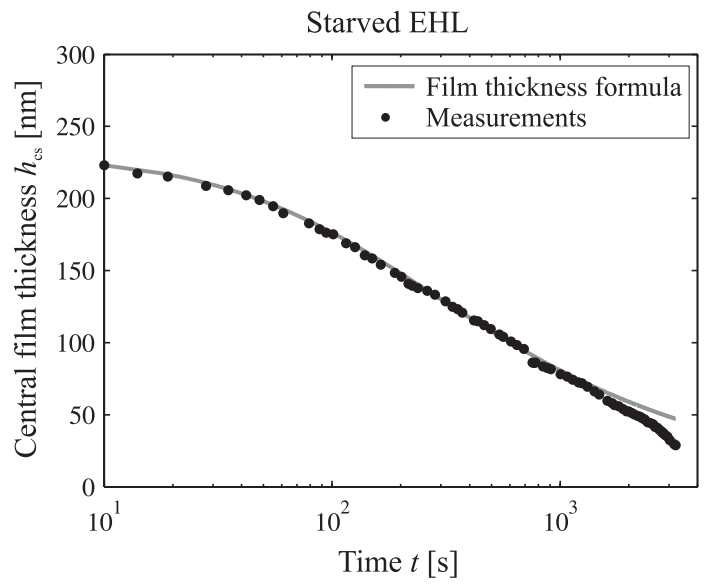

Figure 4.8: Film thickness as function of time for an elliptic EHL contact operating under starved conditions: measured values and prediction by the model presented in Chapter 5 . 


\section{Chapter 5}

\section{FILM THICKNESS DECAY IN STARVED}

\section{EHL CONTACTS}

In this chapter the effect of the EHL contact pressure on the thickness of the lubricant layers on the running track is studied. A model is presented for the prediction of the layer thickness decay as a function of time, when reflow effects are negligible. Both circular and elliptic EHL contacts are considered. As the film thickness inside a starved EHL contact is determined by the thickness of the layers supplied to the contact, this model can be used to predict the film thickness decay. The main part of this research has been published in van Zoelen et al. [79].

\subsection{Theoretical formulation}

In standard lubrication models, the reference frame is fixed to the lubricant film and the analysis is restricted to the region around the pressurized zone of the EHL contact. In the present work a different approach is taken. The modeling frame includes each of the rolling tracks on the running surfaces. The tracks are assumed to be covered by a thin layer of lubricant. This layer is continually forced by the pressure distribution in the EHL contact(s),
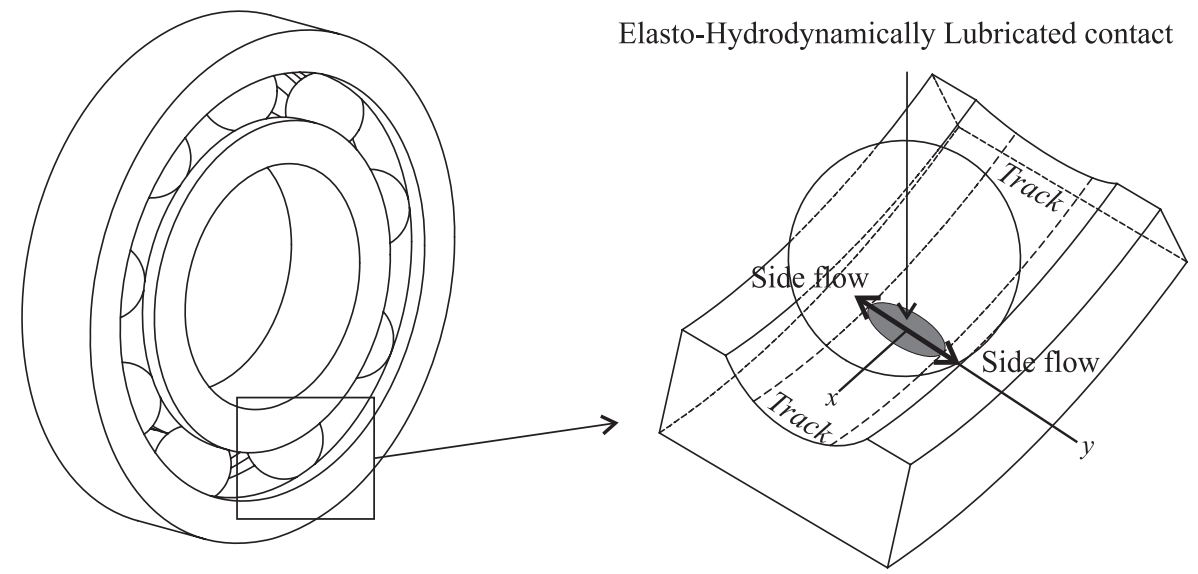

Figure 5.1: Ball bearing section, illustrating side flow in a single elasto-hydrodynamically lubricated contact, expelling lubricant from the track to the sides. 
causing the lubricant to flow to the sides of the track, see Figure 5.1. The main idea of the present model is to quantify the flow perpendicular to the rolling direction, i.e. the side flow, and use the side flow to predict the decrease of the supply layer thickness on the tracks. In 1973 Kingsbury [44, 45] already developed a simple model for the film thickness in a starved EHL contact based on the side flow in the contact. However, in this model he did not consider the amount of lubricant on the tracks and the relation between the layer thickness and film thickness. Also, the assumptions in the derivation of the side flow are rather crude.

In the sections 5.1.1 and 5.1.2 the present layer thickness model is described. Inputs to the model are the starved EHL film thickness and contact pressure distribution. These can be obtained from solving the starved EHL contact problem numerically. However, in section 5.1.3 it will be shown that, for severely starved cases, it is sufficient to use the Hertzian semi-ellipsoid as an approximation of the pressure distribution in the contact, resulting in a simplified layer thickness equation presented in section 5.1.4. This equation is used to derive an equation for the central film thickness as a function of time in section 5.1.5.

\subsubsection{Layer thickness model}

In Figure 5.2, a schematic representation of a part of the rolling track is shown, including an elliptic contact area, representing the starved EHL pressurized region. Pure rolling is assumed and the rolling is in the $x$-direction. $a^{+}(y)$ and $a^{-}(y)$ denote the location of the boundaries of the pressurized region on the inlet side (upstream) and the outlet side (downstream), respectively. The Cartesian coordinates $x$ and $y$ originate at the centre of the Hertzian region and the coordinates $s$ and $\theta$ are fixed to the track. In this chapter the coordinate $y$ is used to describe a position along the width of the track and the coordinate $s$, used in the centrifugal effects model, is replaced by $y+\frac{1}{2} L$.

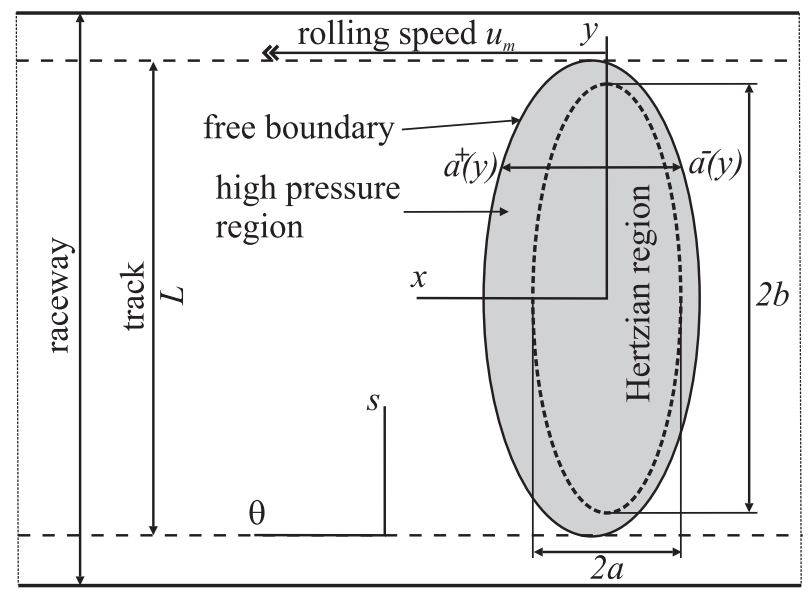

Figure 5.2: Schematic representation of a section of the rolling track and an elliptical contact operating in the starved regime. 
In section 3.1.1 a relation has been derived for the layer thickness $h_{\infty}(s, t)$ averaged over the circumference of the rollers and the inner and outer raceways, see Eq. (3.7). Replacing $s$ by $y+\frac{1}{2} L$ this relation reads:

$$
\frac{\partial \tilde{h}_{\infty}}{\partial t}+\frac{1}{l_{t} \rho_{0}} \frac{\partial \hat{q}_{y}}{\partial y}=0 .
$$

In the model presented in this chapter the track length $l_{t}(y)$ will be approximated by its central value, thus $l_{t}=l_{t}(y=0)$. Using Eq. (5.1) the layer thickness distribution averaged over the total track length $\tilde{h}_{\infty}(y, t)$ can be calculated when the rate of mass flow to the side integrated over the total track length $\hat{q}_{y}(y, t)$ is known. In this chapter only the effect of the contact pressure on the supply layer thickness is considered. In that case the rate of mass flow $\hat{q}_{y}$ is determined by the sum of the contributions of each of the EHL contacts, numbered by index $k$, in the configuration:

$$
\hat{q}_{y}(y, t)=\sum_{k=1}^{n_{c}} \hat{q}_{y, k}(y, t),
$$

where $n_{c}$ denotes the total number of EHL contacts. The rate of mass flowing to the side for a single EHL contact $k$ is deduced from the Reynolds equation (4.11):

$$
q_{y, k}(x, y, t)=-\frac{\rho h^{3}}{12 \eta} \frac{\partial p}{\partial y} .
$$

The total mass flow rate to the side for contact $k$ at time $t$ and position $y$ is obtained by integration of Eq. (5.3) over the contact area in $x$ direction:

$$
\hat{q}_{y, k}(y, t)=-\int_{a^{-}}^{a^{+}}\left(\frac{\rho h^{3}}{12 \eta} \frac{\partial p}{\partial y}\right) d x,
$$

where the film thickness $h=h(x, y, t)$. The density $\rho$ and viscosity $\eta$ are a function of the pressure $p=p(x, y, t)$, which are modeled using the empirical relations presented by Dowson and Higginson, Eq. (4.18), and Roelands, Eq. (4.16), respectively. Thus, the mass flow rate $\hat{q}_{y, k}$ can be computed, when the pressure $p$ and the film thickness $h$ are known.

For a starved EHL contact the film thickness $h$ depends on the sum of the thickness of the layers supplied to the contact $h_{\text {oil }}$. As in the centrifugal force effect model, it is assumed that in the outlet of an EHL contact the lubricant equally partitions between the contacting surfaces. The continuous exchange of liquid in the roller raceway contacts, together with other effects such as contact pressure and surface tension, will tend to distribute the lubricant evenly along the circumference of the contacting solid surfaces. As a result, after many equipartitions the thickness of the lubricant layer on the tracks $\tilde{h}(\theta, y, t)$ is equal to the layer thickness distribution averaged over the track length $\tilde{h}_{\infty}(y, t)$. An example of such a distribution is shown in Figure 5.3 for a spherical thrust bearing. For a ball bearing this distribution only occurs when the rolling elements do not spin. The effect of the cage on the distribution is not taken into account here. 


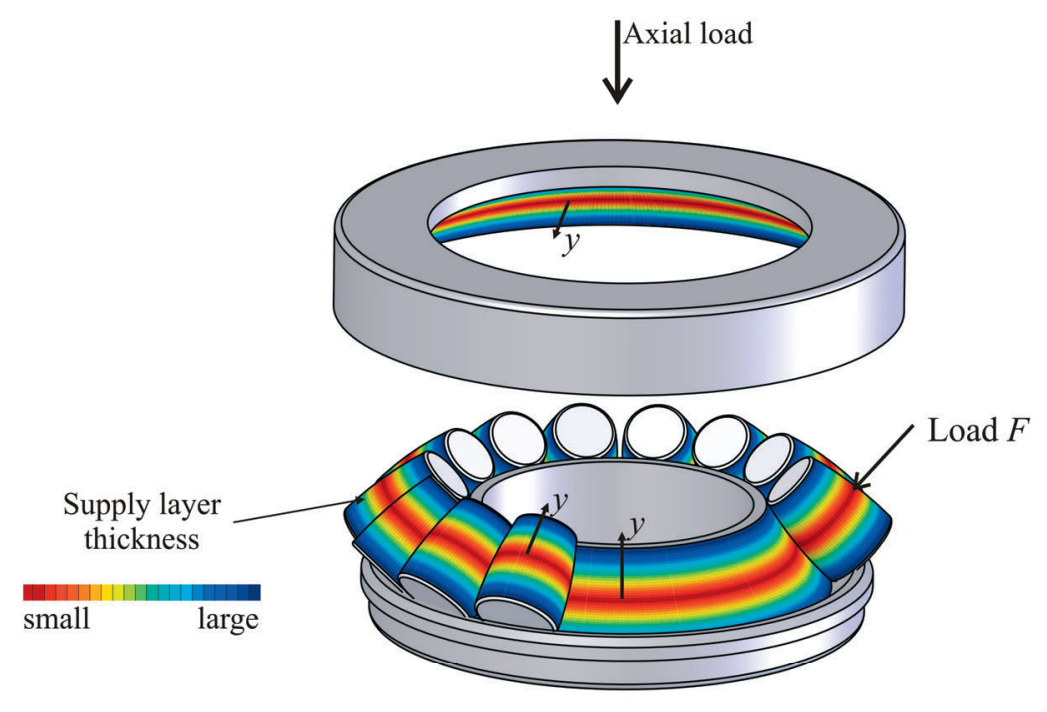

Figure 5.3: Schematic representation of a spherical thrust bearing operating in the starved regime, illustrating that for the long term operation the supply layer thickness distribution and thus the inlet condition $h_{\text {oil }}$ is similar for each contact. For pure axial loading the load on each roller is similar.

For the conditions assumed, the sum of the thickness of the layers close to the inlet $h_{\text {oil }}$ for each contact may be approximated as twice the average free surface layer thickness:

$$
h_{\text {oil }}(y, t) \approx 2 \tilde{h}_{\infty}(y, t) \text {. }
$$

Thus, the mass flow rate $\hat{q}_{y, k}$ in each contact depends on the same inlet condition $h_{\text {oil }}$.

In a bearing the pressure may not be equal for each EHL contact. For example in a radially loaded bearing the load and thus the pressure varies over the circumference of the bearing. In that case the mass flux $\hat{q}_{y, k}$ is also a function of the circumference of the bearing. Furthermore, the pressure depends on the geometry of the steel surfaces, which can be different for the inner and outer raceway. The effect of the load distribution and the difference of the inner/outer raceway geometry on the mass flow $\hat{q}_{y}$ will be modelled in Chapter 6. For now it is assumed that contact geometry and the load are equal for each contact. This occurs for example in purely axially loaded thrust bearings, see Figure 5.3. As the inlet condition $h_{\text {oil }}$ is similar for each contact, for this case the film thickness, the pressure and thus the side flow rate is equal for each contact. Therefore, Eq. (5.2) becomes:

$$
\hat{q}_{y}(y, t)=n_{c} \hat{q}_{y, k}(y, t) .
$$

For a single contact situation such as in the ball and disk apparatus $n_{c}=1$ and the mass flow rate to the side is $\hat{q}_{y}=\hat{q}_{y, k}$. 


\subsubsection{Dimensionless equations}

The equations are made dimensionless introducing the following dimensionless variables:

$$
\begin{aligned}
& X=x / b, \quad Y=y / b, \quad \bar{a}^{+}=a^{+} / a, \quad \bar{a}^{-}=a^{-} / a, \quad \bar{\rho}=\rho / \rho_{0}, \\
& \bar{\eta}=\eta / \eta_{0}, \quad P=p / p_{h}, \quad H=h / c, \quad \tilde{H}_{\infty}=\tilde{h}_{\infty} / c, \quad H_{\text {oil }}=h_{\text {oil }} / c, \\
& \hat{Q}_{Y}=\hat{q}_{y} \frac{12 \eta_{0} b}{n_{c} h_{\text {oil }}^{3} \rho_{0} p_{h} a}, \quad \hat{Q}_{Y, k}=\hat{q}_{y, k} \frac{12 \eta_{0} b}{h_{\text {oil }}^{3} \rho_{0} p_{h} a}, \quad \text { and } \quad \bar{t}=t / \tau .
\end{aligned}
$$

For the definition of the variables the reader is referred to the nomenclature and Chapter 4. The time scale is defined by:

$$
\tau=\frac{3 \eta_{0} l_{t} b^{2}}{2 n_{c} c^{2} p_{h} a} .
$$

Scaling of Eq. (5.1), Eq.(5.4) and Eq. (5.6) gives:

$$
\begin{gathered}
\frac{\partial \tilde{H}_{\infty}}{\partial \bar{t}}+\frac{\partial}{\partial Y}\left(\frac{1}{8} H_{o i l}^{3} \hat{Q}_{Y}\right)=0, \\
\hat{Q}_{Y, k}(Y, \bar{t})=\int_{\bar{a}^{-}}^{\bar{a}^{+}}\left(-\frac{H^{3}}{H_{\text {oil }}^{3}} \frac{\bar{\rho}}{\bar{\eta}} \frac{\partial P}{\partial Y}\right) d X,
\end{gathered}
$$

and:

$$
\hat{Q}_{y}(Y, \bar{t})=\hat{Q}_{y, k}(Y, \bar{t}),
$$

where $H=H(X, Y, \bar{t})$. Due to the scaling, the total dimensionless thickness of the supply layers just in front of the inlet to the contact $H_{o i l}(Y, \bar{t})$ is introduced in the equations. $Q_{Y, k}$ is the dimensionless mass flow to the side in contact $k$ divided by $H_{\text {oil }}^{3}$. The dimensionless density $\bar{\rho}$ and viscosity $\bar{\eta}$ are a function of the dimensionless pressure $P=P(X, Y, \bar{t})$, which are modeled using Eq. (4.25) and Eq. (4.27), respectively. Equation (5.5) in dimensionless variables reads:

$$
H_{o i l}(Y, \bar{t}) \approx 2 \tilde{H}_{\infty}(Y, \bar{t}) .
$$

The dimensionless layer thickness equation is obtained by substitution of Eq. (5.12) into Eq. (5.9):

$$
\frac{\partial \tilde{H}_{\infty}}{\partial \bar{t}}+\frac{\partial}{\partial Y}\left(\tilde{H}_{\infty}^{3} \hat{Q}_{Y}\right)=0
$$


Considering a symmetrical distribution of $\tilde{H}_{\infty}(Y, \bar{t})$ with respect to the $X$ axis, the solution for the track centreline $Y=0$ reads:

$$
\tilde{H}_{\infty}(0, \bar{t})=\left(2 \int_{0}^{\bar{t}}\left(\frac{\partial \hat{Q}_{Y}}{\partial Y}\right)_{Y=0} d \bar{t}+\tilde{H}_{0, \infty}^{-2}\right)^{-1 / 2},
$$

where $\tilde{H}_{0, \infty}$ is the initial layer thickness along the symmetry line $Y=0$.

Solving Eq. (5.13) or Eq. (5.14) for $\tilde{H}_{\infty}$ and some initial condition involves an iterative process, as $\hat{Q}_{Y}$ is a function of the layer thickness itself. The calculation of $\hat{Q}_{Y}$ requires $H$ and $P$, which can be obtained by solving the starved EHL problem numerically, with the inlet condition $H_{\text {oil }}=2 \tilde{H}_{\infty}$. Also, in the Elastic Piezoviscous regime, Ertel-Grubin like methods [52] can be used to approximate $H$ and $P$ in the inlet to the contact at $Y=0$. In addition, in the next section it will be shown that for the case of heavily starved contacts, $\hat{Q}_{Y}$ can be calculated, with good accuracy, using the Hertzian contact pressure and gap width as an approximation. In this way, without having to solve the complete starved lubrication problem, a prediction of the film thickness decay after many overrollings can be obtained.

\subsubsection{Asymptotic dimensionless flux}

In Figure 5.4 a cross section of the numerically simulated film thickness and pressure distribution at $Y=0$ and $X=0$ are shown, for a circular EHL contact operating under fully flooded conditions. In addition, three starved cases are shown with different inlet layer thickness, relative to the central fully flooded film thickness: $H_{\text {oil }} / H_{\text {cff }}=1, \frac{1}{2}$, and $\frac{1}{4}$. Also, the Hertzian pressure distribution is shown. The values of the parameters for this case are: $N=20.1, L=10.4, D=1, \eta_{0}=0.8$ Pa.s, and $\alpha=20 \cdot 10^{-9} \mathrm{~Pa}^{-1}$.

From the studies of single starved contacts (Chevalier et al. [18], Damiens et al. [22]) it is known that, in the heavily starved lubrication regime the film thickness inside the contact is directly related to the layer thickness supplied to the contact. In Figure 5.4, it can be seen that with increasing degree of starvation (decreasing $H_{\text {oil }}$ ), the central film thickness decreases. Moreover, the film thickness distribution inside the contact becomes more uniform. The gap height $H$ converges to:

$$
H(Y, \bar{t})=\frac{H_{o i l}(Y, \bar{t})}{\bar{\rho}(P)} .
$$

At the same time the pressure distribution becomes more narrow, i.e. the extent of the inlet region towards the contact decreases. The location of the boundary of the pressurized region approaches the Hertzian contact circle. In that case the dimensionless location of the pressure boundary is defined by:

$$
\bar{a}^{+}=-\bar{a}^{-}=\sqrt{1-Y^{2}},
$$


while the pressure distribution approaches the Hertzian dry contact pressure distribution $\left(P_{H}\right)$. This applies to both the direction of motion ( $X$-direction) and the cross-flow direction ( $Y$-direction). Thus, the dimensionless pressure distribution $P$ converges to:

$$
P=P_{H}=\sqrt{1-X^{2}-Y^{2}}
$$
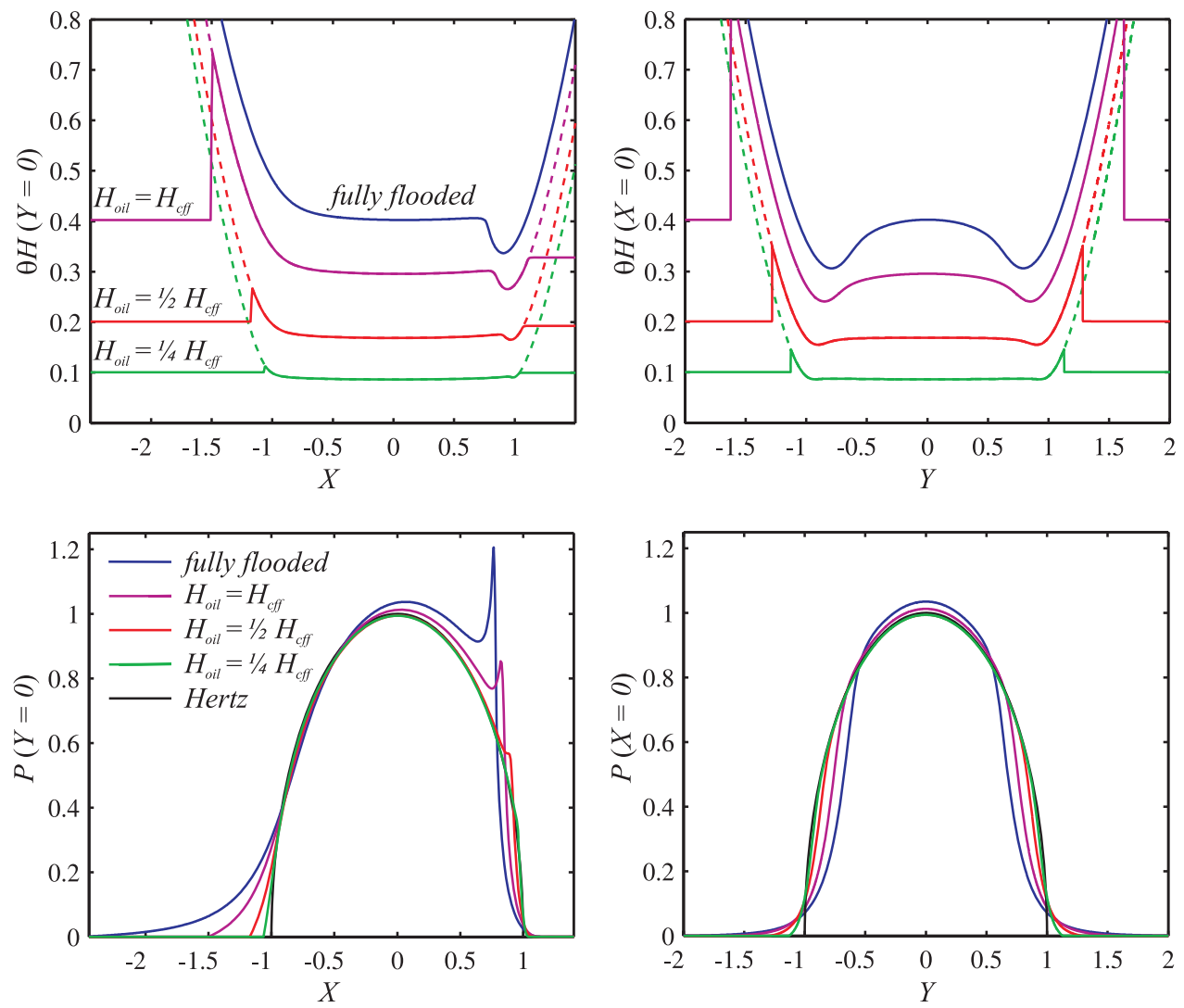

Figure 5.4: Film thickness and pressure distribution for various values of $H_{\text {oil }}$ and fully flooded conditions for $D=20.1, N=20.1$ and $L=10.4$ in dimensionless variables. See also Figure 4.4. 
Substitution of Eq. (5.10), Eq. (5.15), Eq. (5.16) and Eq. (5.17) in Eq. (5.11), and introduction of a new variable $\varphi$, gives the value of $\hat{Q}_{Y}$ for the asymptotic regime (See Appendix C.1 for a detailed derivation):

$$
\hat{Q}_{Y}(Y)=Y C(Y),
$$

with:

$$
C(Y)=\int_{-1 / 2 \pi}^{1 / 2 \pi}\left(\left(\bar{\rho}\left(P_{H}\right)\right)^{-2}\left(\bar{\eta}\left(P_{H}\right)\right)^{-1}\right) d \varphi,
$$

and:

$$
P_{H}(\varphi, Y)=\sqrt{1-Y^{2}} \cos (\varphi) .
$$

Input to the equation for the central layer thickness (Eq. (5.14)) is the gradient $\partial \hat{Q}_{Y} / \partial Y$ at $Y=0$, which for the asymptotic regime is obtained by differentiation of Eq. (5.18):

$$
\lim _{H_{\text {oil }} \downarrow 0}\left(\frac{d \hat{Q}_{Y}}{d Y}\right)_{Y=0}=C(0) .
$$

In Figure 5.5 the ratio is shown of the flux gradient $\partial \hat{Q}_{Y} / \partial Y$ at $Y=0$, calculated numerically using the starved EHL solver, and the asymptotic flux gradient $C(0)$ as a function of the inlet layer thickness $H_{\text {oil }} / H_{\text {cff }}$. The same parameters are used as for the results of Figure 5.4. Note that the ratio approaches 1.0 for thinner inlet layers. Hence, the numerically obtained flux gradient indeed converges to the asymptotic flux gradient with an increasing degree of starvation.

This is further illustrated in Table 5.1, where the value of $\partial \hat{Q}_{Y} / \partial Y$ at $Y=0$ is given for different cases represented by the dimensionless Moes parameters $N, L$, and $D$, with $\alpha=20 \mathrm{GPa}^{-1}$ and $\eta_{0}=0.8$ Pa.s and inlet layer thickness $H_{\text {oil }} / H_{\text {cff }}$. For more information regarding the numerical accuracy of the values the reader is referred to appendix C.2. The asymptotic values of $\partial \hat{Q}_{Y} / \partial Y$ are calculated using Eq. (5.21) with Eq. (5.19) and Eq. (5.20). Notice that also for these cases the numerically calculated values converge to the asymptotic case with decreasing thickness of the inlet layer $H_{\text {oil }} / H_{\text {cff }}$. For thicker inlet layers the deviation from the asymptotic value increases with increasing $N$ and decreasing $D$. 


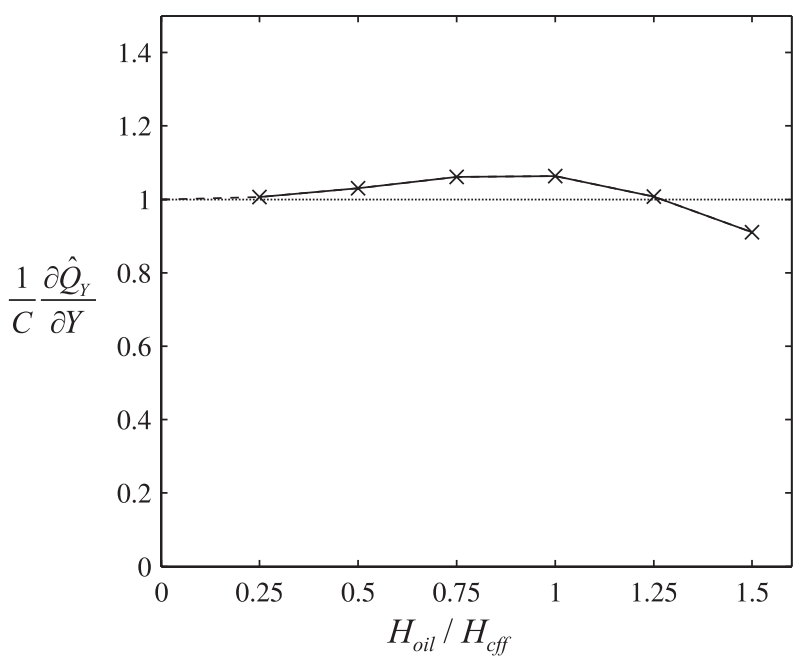

Figure 5.5: Ratio of the numerically obtained flux gradient $\partial \hat{Q}_{Y} / \partial Y$ and the asymptotic flux gradient $C$ as a function of the inlet layer thickness for $Y=0, N=20.1, L=10.4$ $\eta_{0}=0.8$ Pa.s, and $\alpha=20 \cdot 10^{-9} \mathrm{~Pa}^{-1}$. The ratio approaches 1.0 for smaller inlet layer thicknesses.

\begin{tabular}{|c|c|c|c|c|c|c|c|}
\hline$N$ & $L$ & $D$ & $H_{c f f}$ & \multicolumn{4}{|c|}{$\partial \hat{Q}_{Y} / \partial Y$ at $Y=0$} \\
\cline { 5 - 8 } & & & & $\frac{H_{\text {oil }}}{N}=1$ & $\frac{H_{\text {oil }}}{H_{\text {cff }}}=\frac{1}{2}$ & $\frac{H_{\text {oil }}}{H_{\text {cff }}}=\frac{1}{4}$ & $\lim _{H_{\text {oil }} \downarrow 0}$ \\
\hline 20 & 2.5 & 1 & $2.3110^{-1}$ & 0.884 & 0.911 & 0.917 & 0.924 \\
\hline 20 & 5.0 & 1 & $3.0310^{-1}$ & 0.481 & 0.477 & 0.467 & 0.467 \\
\hline 20 & 10 & 1 & $4.2110^{-1}$ & 0.237 & 0.233 & 0.227 & 0.225 \\
\hline 100 & 2.5 & 1 & $7.1110^{-2}$ & 0.763 & 0.590 & 0.559 & 0.550 \\
\hline 100 & 5.0 & 1 & $9.4310^{-2}$ & 0.406 & 0.300 & 0.273 & 0.265 \\
\hline 100 & 10 & 1 & $1.3310^{-1}$ & 0.199 & 0.151 & 0.132 & 0.129 \\
\hline 1000 & 2.5 & 1 & $1.2110^{-2}$ & 0.582 & 0.281 & 0.248 & 0.245 \\
\hline 1000 & 5.0 & 1 & $1.6410^{-2}$ & 0.307 & 0.150 & 0.126 & 0.120 \\
\hline 1000 & 10 & 1 & $2.3710^{-2}$ & 0.150 & 0.077 & 0.065 & 0.060 \\
\hline 100 & 10 & 0.1 & $1.1810^{-1}$ & 0.747 & 0.226 & 0.201 & 0.167 \\
\hline 100 & 10 & 0.01 & $9.9210^{-2}$ & 1.223 & 0.280 & 0.256 & 0.187 \\
\hline
\end{tabular}

Table 5.1: Numerically obtained values of $\partial \hat{Q}_{Y} / \partial Y$ for $Y=0, \quad \eta_{0}=0.8$ Pa.s, $\alpha=20 \cdot 10^{-9} \mathrm{~Pa}^{-1}$ and various values of $N, L$ and $D$. The limit is calculated using Eq. (5.21) employing Eq. (5.19) and Eq. (5.20). 


\subsubsection{Solution layer thickness equation}

When the asymptotic flux (5.18) is substituted into Eq. (5.13) the resulting layer thickness equation is similar to Eq. (3.10), as in that case $\hat{Q}_{Y}$ is independent of the time. Hence, Eq. (5.13) can be solved using the method of characteristics, see Appendix A.1. The solution for the dimensionless layer thickness along a characteristic reads:

$$
\tilde{H}_{\infty}(Y, \bar{t})=\left(\frac{Y_{0} C\left(Y_{0}\right)}{Y C(Y)}\right)^{1 / 3} \tilde{H}_{\infty, 0},
$$

where $\tilde{H}_{\infty, 0}=\tilde{H}_{\infty, 0}\left(Y_{0}\right)$ is the initial layer thickness distribution and $Y_{0}$ follows from the numerical solution of the equation:

$$
\int_{Y_{0}}^{Y}(\phi C(\phi))^{-1 / 3} d \phi=3\left(Y_{0} C\left(Y_{0}\right)\right)^{2 / 3} \tilde{H}_{\infty, 0}^{2} \bar{t},
$$

for a particular $Y$ and $\bar{t}$. In the calculations of the layer thickness presented in this chapter, a recursive adaptive Simpson quadrature approximation to the integral of Eq. (5.23) was used with an absolute error tolerance of $10^{-6}$. The resulting equation was solved iteratively, with a termination tolerance of approximately $2 \cdot 10^{-16}$. Before solving Eq. (5.23) the function $C(Y)$ is calculated and stored for 1600 uniformly distributed grid points in $Y$. Subsequently, in the process of iteratively solving $Y_{0}, C\left(Y_{0}\right)$ is determined by linear interpolation from these pre-computed values.

The central layer thickness for the asymptotic regime is obtained by substitution of Eq. (5.21) into Eq. (5.14):

$$
\tilde{H}_{\infty}(0, \bar{t})=\left(2 C(0) \bar{t}+\tilde{H}_{\infty, 0}^{-2}\right)^{-1 / 2}
$$

\subsubsection{Film thickness}

The relation between the film thickness and the layer thickness in the asymptotic regime $\left(H_{\text {oil }} \downarrow 0\right)$ is obtained by substitution of Eq. (5.12) into Eq. (5.15):

$$
H(Y, \bar{t})=\frac{2 \tilde{H}_{\infty}(Y, \bar{t})}{\bar{\rho}\left(P_{H}\right)} .
$$

Combining Eq. (5.25) and Eq. (5.24) gives central film thickness as a function of time for the asymptotic regime:

$$
H_{c s}(\bar{t})=\left(\frac{1}{2} \bar{\rho}_{c}^{2} C(0) \bar{t}+H_{c s, 0}^{-2}\right)^{-1 / 2},
$$

where $\bar{\rho}_{c}=\bar{\rho}(P=1)$ is the dimensionless density of the lubricant in the center of the contact and $H_{c s, 0}$ is the central film thickness at $\bar{t}=0$. 
When the Dowson and Higginson density-pressure relation (4.25) and the Roelands equation (4.27) are used the dimensionless film thickness for the asymptotic regime is fully defined by the pressure-viscosity coefficient $\alpha$, the maximum Hertzian pressure $p_{h}$, and the dynamic viscosity at ambient pressure $\eta_{0}$.

\subsection{Theoretical results}

\subsubsection{Layer thickness distribution}

In Figure 5.6 the solution is shown for the layer thickness distribution across the width of the track for various times. The results are shown in dimensionless variables. The EHL contact parameters are: $N=100, L=10$, and $D=0.1$, with $\alpha=20 \mathrm{GPa}^{-1}$, and $\eta_{0}=0.8 \mathrm{~Pa} \cdot \mathrm{s}$. For these parameters the pressure is $p_{h}=0.66 \mathrm{GPa}$ and the dimensionless fully flooded central film thickness is $H_{c f f}=0.118$. The initial layer thickness distribution is uniform with a thickness of $\tilde{H}_{0, \infty}=\frac{1}{2} H_{c f f}$.

Recall that this result is valid for the situation of a single contact at a constant load, or the situation of multiple contacts, where the contact geometry and the load are equal for each contact. This occurs for example in a purely axially loaded thrust bearing, see Figure 5.3. The results show a relatively rapid layer thickness decrease near the boundaries of the track. This difference in layer thickness is a result of the relatively large viscosity due to high pressures in the center region of the contact. As a consequence, the side flow rate is reduced in the central region, resulting in a slower decreasing layer thickness at this location.

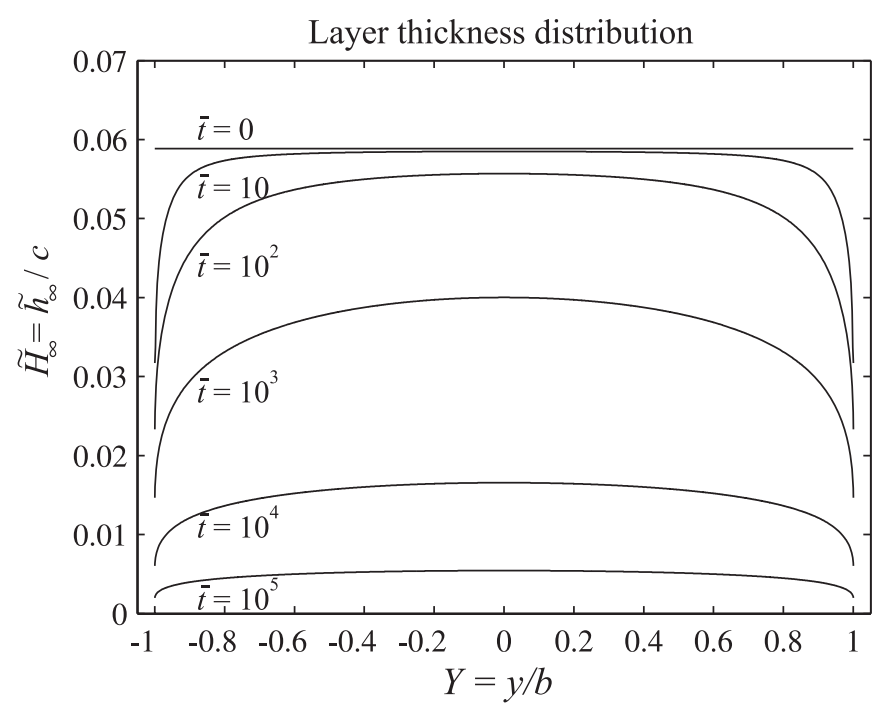

Figure 5.6: Layer thickness distribution at various times in dimensionless variables, with $p_{h}=0.66 \mathrm{GPa}, \eta_{0}=0.8 \mathrm{~Pa} \cdot \mathrm{s}$ and $\alpha=20 \cdot 10^{-9} \mathrm{~Pa}^{-1}$. 


\subsubsection{Central layer thickness}

In Figure 5.7 the layer thickness as a function of time at $Y=0$ is shown in dimensionless variables for the case discussed in the preceding section. Two curves are shown:

1. The solid line represents the solution calculated from Eq. (5.14), using the values of $\partial \hat{Q}_{Y} / \partial Y$ obtained from the solution of the complete starved lubrication problem for different degrees of starvation, see Table 5.1. A linear interpolation is used to calculate $\partial \hat{Q}_{Y} / \partial Y$ at the intermediate values of $H_{\text {oil }}=2 \tilde{H}_{\infty}$. Equation (5.14) is calculated using discrete time steps $k$ with length equal $\Delta \bar{t}$ :

$$
\tilde{H}_{\infty}^{k+1}=\left(2 \Delta \bar{t} \sum_{k^{\prime}=0}^{k}\left(\frac{\partial \hat{Q}_{Y}}{\partial Y}\left(\tilde{H}_{\infty}^{k^{\prime}}\right)\right)+\tilde{H}_{0, \infty}^{-2}\right)^{-1 / 2} .
$$

2. The dashed line represents the solution that is calculated using the layer thickness equation for the asymptotic regime (5.24).

Note that even though the asymptotic and numerically calculated $\hat{Q}_{Y}$ for thicker layers differ quite significantly (see Table 5.1), for larger times $\bar{t}$ approximately the same layer thickness is predicted.

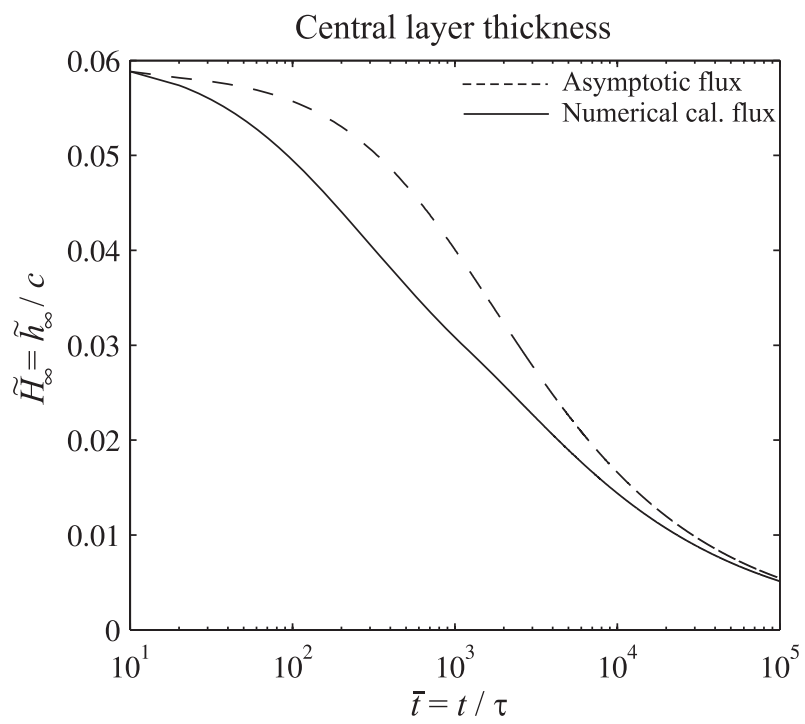

Figure 5.7: Decreasing layer thickness in time in dimensionless variables, with $N=100$, $L=10, D=0.1, \alpha=20 \mathrm{GPa}^{-1}$, and $\eta_{0}=0.8 \mathrm{~Pa} \cdot \mathrm{s}$. The solid line is the solution calculated from Eq. (5.27) using a linear interpolation of the numerically calculated flux values given in Table 5.1. The dashed line is the solution of the asymptotic film thickness decay equation (5.24). 


\subsubsection{Influence of physical parameters}

In this section the influence of the physical parameters on the central starved film thickness for the asymptotic regime is discussed. Equation (5.26) in dimensional form reads:

$$
h_{c s}(t)=\left(C_{2} t+h_{c s, 0}^{-2}\right)^{-1 / 2}
$$

with:

$$
C_{2}=\frac{\frac{1}{2} \bar{\rho}_{c}^{2} C}{\tau c^{2}}=\frac{n_{c} p_{h} a \bar{\rho}_{c}^{2} C}{3 \eta_{0} l_{t} b^{2}}=\frac{n_{c} E^{\prime} \kappa^{2} \bar{\rho}_{c}^{2} C}{12 \eta_{0} l_{t} R \mathcal{E}},
$$

where $C=C(0)$. The asymptotic decay rate of the central film thickness is determined by parameter $C_{2}$, and, according to Eq. (5.29), the decay rate is smaller for a larger viscosity $\eta_{0}$ and a larger total track length $l_{t}$ and it is independent of the velocity $u_{m}$. Furthermore, for a particular pressure $p_{h}$, the decay rate is larger for a larger reduced modulus of elasticity $E^{\prime}$, for a larger number of contacts $n_{c}$, and for a larger value of the geometry parameter group $\kappa^{2} / R \mathcal{E}$.

The parameter group $\bar{\rho}_{c}^{2} \mathrm{C}$ is a decreasing function of $p_{h}$, as is shown in Figure 5.8 for $\eta_{0}=0.8 \mathrm{~Pa} \cdot \mathrm{s}$ and $\alpha=20 \cdot 10^{-9} \mathrm{~Pa}^{-1}$. The Hertzian pressure can be written as:

$$
p_{h}=\sqrt[3]{\frac{3 F \kappa E^{\prime 2}}{32 \pi R^{2} \mathcal{E}^{2}}}
$$

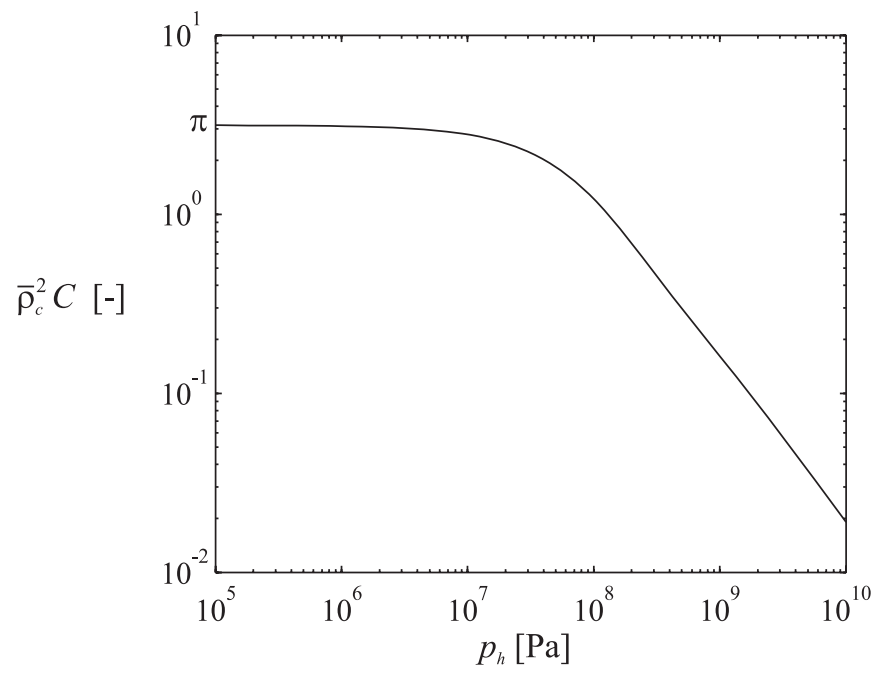

Figure 5.8: Parameter group $\bar{\rho}_{c}^{2} C$ as a function of the pressure $p_{h}$, for $\eta_{0}=0.8$ and $\alpha=20 \cdot 10^{-9} \mathrm{~Pa}^{-1}$. 
According to Eq. (5.30), Figure 5.8 and Eq. (5.29), a higher load $F$ gives a lower value of $C_{2}$, and thus a smaller film thickness decay rate. This behavior is quite remarkable and indicates that optimal design of lubricated contacts, i.e. maximizing the lubricant film, in the case of starved contacts may not necessarily mean minimizing the load. Indeed, during the initial operation the contacts operate under fully flooded conditions and in that case the film thickness is smaller at higher loads. However, during operation the contacts become increasingly starved and under severely starved EHL conditions the decay rate is smaller at higher loads. Hence, in the long run the film thickness may be larger. In fact, the contacts are more efficiently using the lubricant supplied for film formation inside the contact. It is stressed that this behavior is not an artifact from the presented model. It can also be seen in the results presented by Damiens, see Figure 4 in [22], although it is not explicitly mentioned there. Also, it is confirmed by the experimental results presented at the end of this chapter. Moreover, it is even already visible in the crude model presented by Kingsbury [44].

\subsection{Experimental approach}

The model is validated using the setup described in Section 4.8. The measurement procedure is as follows. A clean ball or roller is positioned in the rig and the radial position of the contact on the disk is set. Using a syringe, a small droplet of oil is placed on the surface of the rolling element, a few millimetres in front of the contact. The disk is mounted and the spacer layer thickness is determined and set as zero film thickness level. Next, the disk is rotated at a surface velocity in the contact of $u_{m}=300 \mathrm{~mm} / \mathrm{s}$ and run for half an hour to obtain a uniform lubricant distribution along the track.

During the initial period oil levees are formed on both sides of the track. As these levees are relatively large compared to the layer thickness, the reflow of oil onto the track area in the time between overrollings is large. In order to minimize the reflow, the radius of the track on the disk $R_{\text {disk }}$ is adjusted slowly during rolling, pushing the levees on the disk approximately $2 \mathrm{~mm}$ to both sides of the track. This is illustrated in Figure 5.9. Note that this method cannot remove the levees on the roller. Next, the ball is re-positioned at the initial track radius, the desired velocity $u_{m}$ is chosen, and the layer thickness is measured at various times during approximately one hour.

Oil with a high viscosity is used in order to minimize the reflow between overrollings. The properties of the lubricant and the solids are given in Table 5.2. The total track length for the setup is defined by:

$$
l_{t}=2 \pi\left(R_{x, 1}+R_{\text {disk }}\right),
$$

where $R_{x, 1}$ is the radius of the rolling element in the direction of rolling. The viscosity at 40 ${ }^{\circ} \mathrm{C}$ and $100{ }^{\circ} \mathrm{C}$ are obtained from [76] and the Walther formula (see [62]) is used to obtain the viscosity of the oil at the room temperature. The room temperature is recorded at the start of each experiment. 


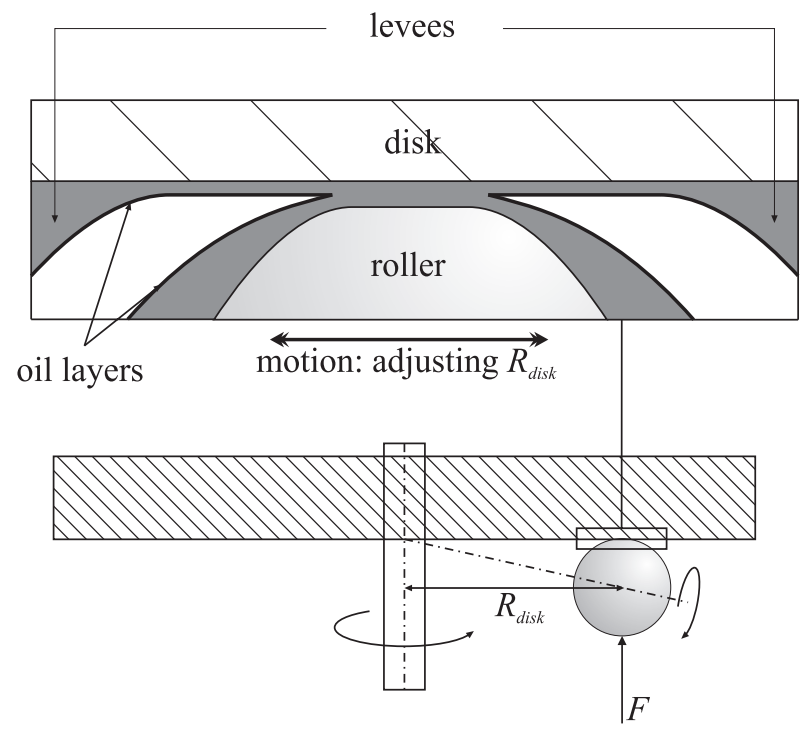

Figure 5.9: Reduction of reflow; the levees are pushed to the sides by slowly adjusting the track radius $R_{\text {disk }}$ during rolling.

\begin{tabular}{|c|c|c|c|c|c|}
\hline \multicolumn{7}{|c|}{ Lubricant type: ExxonMobil SHF403 } \\
\hline \multirow{2}{*}{ Viscosity } & \multirow{2}{*}{$\eta_{0}$} & $40^{\circ} \mathrm{C}$ & \multicolumn{2}{c|}{$100^{\circ} \mathrm{C}$} & \\
\cline { 3 - 6 } & & $3.37 \cdot 10^{-1}$ & \multicolumn{2}{c|}{$3.32 \cdot 10^{-2}$} & Pa.s \\
\hline Density & $\rho_{0}$ & \multicolumn{5}{|c|}{850} & $\mathrm{Kg}^{-3}$ \\
\hline Pressure-viscosity index & $\alpha$ & \multicolumn{5}{c|}{20} & $\mathrm{GPa}^{-1}$ \\
\hline \multicolumn{7}{|c|}{ Properties of the solids } & \\
\hline \multicolumn{7}{|c|}{ Ball } & Roller & Disk & \\
\hline Radius in $x$ dir. & $R_{x, 1}$ & $9.525 \cdot 10^{-3}$ & $9.525 \cdot 10^{-3}$ & $R_{x, 2}=\infty$ & $\mathrm{m}$ \\
\hline Radius in $y$ dir. & $R_{y, 1}$ & $9.525 \cdot 10^{-3}$ & $7.0 \cdot 10^{-2}$ & $R_{x, 2}=\infty$ & $\mathrm{m}$ \\
\hline Reduced radius of curv. & $R$ & $4.762 \cdot 10^{-3}$ & $8.384 \cdot 10^{-3}$ & - & $\mathrm{m}$ \\
\hline Ellipticity ratio & $\kappa$ & 1 & 0.270 & - & - \\
\hline Elliptic integral first & $\mathcal{K}$ & $\pi / 2$ & 2.727 & - & - \\
\hline Elliptic integral sec. & $\mathcal{E}$ & $\pi / 2$ & 1.082 & - & - \\
\hline Modulus of elasticity & $E$ & 210 & 210 & 70 & $\mathrm{GPa}$ \\
\hline Poisson's ratio & $v$ & 0.30 & 0.30 & 0.25 & - \\
\hline Reduced mod. of elas. & $E^{\prime}$ & & 113 & & $\mathrm{GPa}$ \\
\hline
\end{tabular}

Table 5.2: Properties of the lubricant and the solids. 


\subsection{Experimental results}

The experimental results of the film thickness distribution as a function of time will be discussed for the circular contact. Subsequently, the influence of the speed and the load on the central film thickness is studied experimentally for a circular and an elliptical contact. The measurement results are compared with the theoretical predictions.

\subsubsection{Interferometric images}

A video camera is used to record the optical interference images of the contact area between the ball and the disk. In Figure 5.10 six interferometric images are shown captured at the same position on the disk at different times. The disk centre is positioned to the left of the image and the direction of motion of the surfaces is in the positive $x$ direction. The parameters are given in the caption.

Snapshot (a) is recorded immediately after the track radius was slowly adjusted during rolling, as described in section 5.3. Using this method large oil levees at the sides of the track are effectively removed, reducing the reflow from the sides onto the track. The figure shows that the initial profile has a wavy surface. This is the result of the consecutive overrolling of the layer at a varying track radius, i.e. position on $y$. It is found that this waviness disappears during the first overrolling, so it does not influence the film thickness measurement.

Next, the track radius is set to its original position, so the rolling contact is in view of the camera and the first interferometric image of the contact area is recorded (b). The blue coloured area is the high-pressure region, where the classical "horse shoe" shape can be recognized. Its size is approximately equal to the Hertzian contact size. The levees at the sides of the track form a characteristic butterfly shaped meniscus at both sides of the Hertzian region. Here, the oil layer on the ball and the disk are combined into a single oil film. The concentric rings are a result of the increasing gap between the ball and the disk. Above and below the blue area a section of the rolling track on the disk is visible. Notice that the track width in the inlet is slightly smaller than at the outlet. This shows that between two consecutive overrollings reflow occurs at the boundaries of the track.

From the interferometric images film thickness maps can be obtained by image analysis, using a calibration file obtained separately. The central film thickness is given in the figure. For the operating conditions the numerically calculated fully flooded film thickness is $h_{c f f}=463 \mathrm{~nm}$. Accordingly, the contact is already operating in the severely starved EHL regime at $t=0$.

The interferometric images (c), (d) and (e) are recorded at $t=30 \mathrm{~s}, t=112 \mathrm{~s}$ and $t=446 \mathrm{~s}$, respectively. In image (e) the size of the butterfly wing on the right hand side has increased significantly. After image (e) was recorded, it was observed that lubricant occasionally entered the contact from the side, see (f), causing a sudden local increase in film thickness and overall the film thickness did not decay further. 
(a) Intitial oil layer distribution

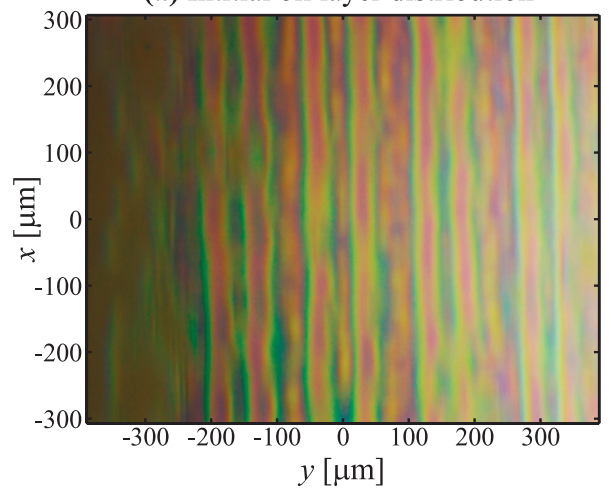

(c) $t=30 \mathrm{sec} . \quad h_{c \mathrm{~s}}=138 \mathrm{~nm}$

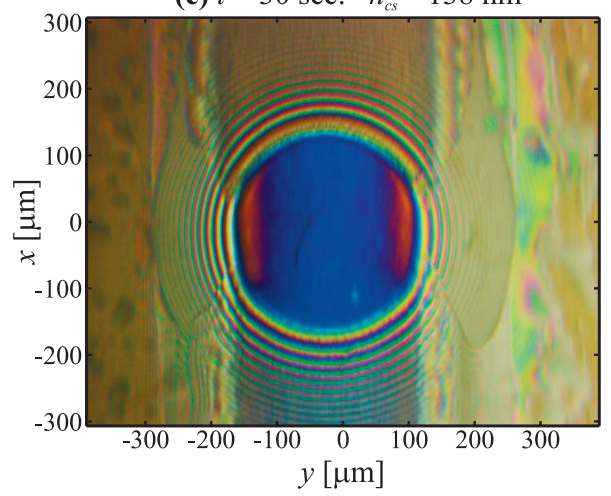

(e) $t=446 \mathrm{sec} . \quad h_{c s}=35 \mathrm{~nm}$

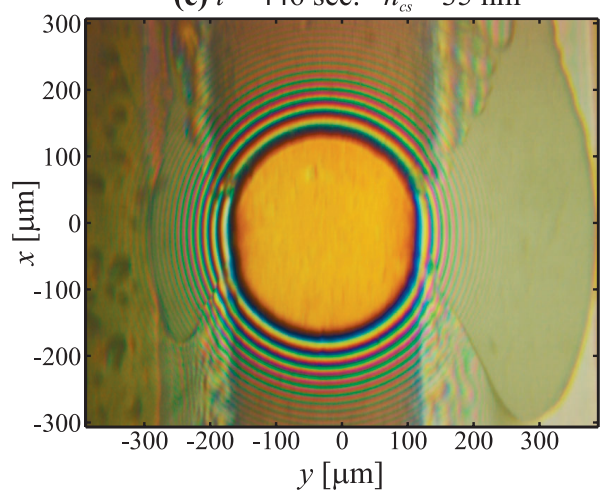

(b) $t=0 \mathrm{sec} . \quad h_{c \mathrm{~s}}=158 \mathrm{~nm}$

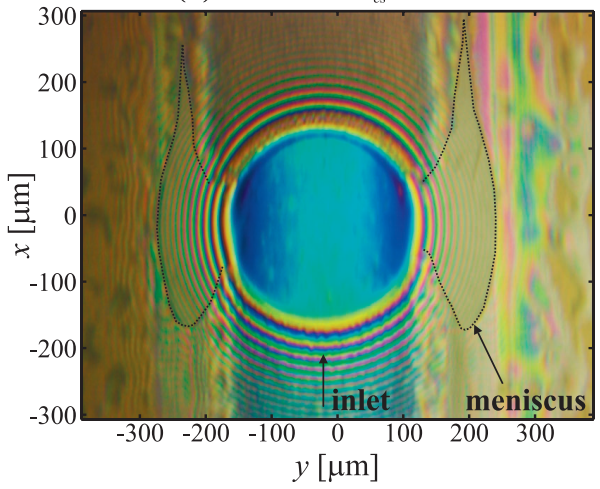

(d) $t=112 \mathrm{sec} . \quad h_{c s}=79 \mathrm{~nm}$

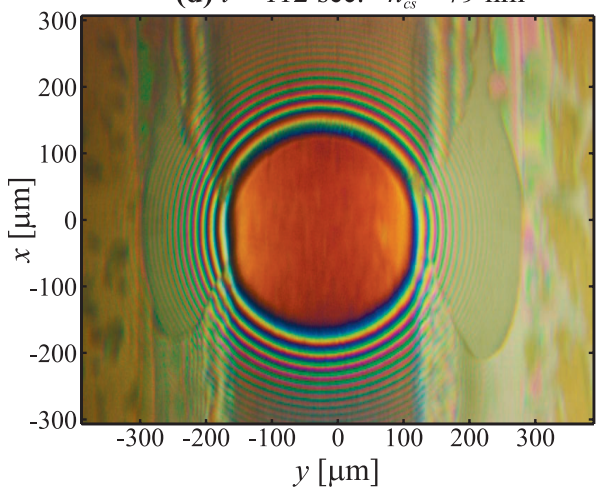

(f) $t=581 \mathrm{sec} . \quad h_{c s}=37 \mathrm{~nm}$

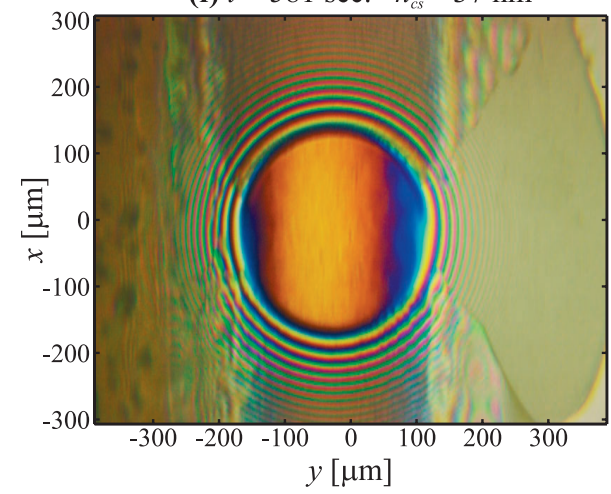

Figure 5.10: Interferometric images of the oil layer/film distribution of an EHL contact operating in the starved EHL regime. Parameters: $u_{m}=107 \mathrm{~mm} / \mathrm{s}, \quad F=19 \mathrm{~N}$, $R_{\text {disk }}=36.5 \mathrm{~mm}, \quad T=22.2^{\circ} \mathrm{C}, \quad \eta_{0}=0.96 \mathrm{~Pa} \cdot \mathrm{s}, \quad p_{h}=0.51 \mathrm{GPa}, \quad a=b=134 \mu \mathrm{m}, \quad N=36$, $L=8.4$, and $h_{c f f}=463 \mathrm{~nm}$. 


\subsubsection{Film thickness distribution}

For the experiment discussed in the preceding section in Figure 5.11 the distributions of the measured film thickness at $x=0$ are shown for 6 instants of time. Also, the results of the model for the asymptotic layer thickness decay are shown in the figure (black lines), where the layer thickness is translated into film thickness by means of Eq. (5.25). In the calculations an initial uniform layer was used with a relatively large thickness. In this way the obtained distribution becomes independent of the initial condition. When the calculated film thickness distribution was equal to the first measured film thickness distribution, the time was set to zero.

The results show that the measured film thickness in the central region is larger than predicted by the model, but the general decay trend is predicted quite well. The minimum film thickness indeed occurs at both sides until $t=143 \mathrm{~s}$. The area where the minimum film thickness occurs tends to spread to the center. At $t=446 \mathrm{~s}$ the distribution is more or less flat. At the left and right boundary the film thickness increases, probably due to reflow from the levees at the side of the track or due to a small amount of spin of the ball. The model predicts a smaller film thickness at the boundaries.

The experiment was repeated at a different track radius $R_{\text {disk }}$. The results are shown in Figure 5.12. The experimental results shown in Figure 5.11 and Figure 5.12 are remarkable similar. An important difference is that the equilibrium film thickness reached in the long term is smaller $\left(h_{c}=27 \mathrm{~nm}\right)$ than in the first experiment $\left(h_{c}=35 \mathrm{~nm}\right)$. This may be a result of the difference in track radius. The support of the ball is designed to prevent spin motion of the ball or sideward slip of the contact. Ideally the rotational axis of the ball should cross the center of the disk at the surface, see Figure 5.9 on page 87. However, in practice there is an alignment error and therefore small sideward slip or spin motion occurs. This results in a small change of the location of the track, effectively allowing lubricant from the levees to enter the contact area. Possibly the alignment error in the second experiment was smaller, as it was observed that the occasionally occurring reflow of lubricant from the side was smaller. 


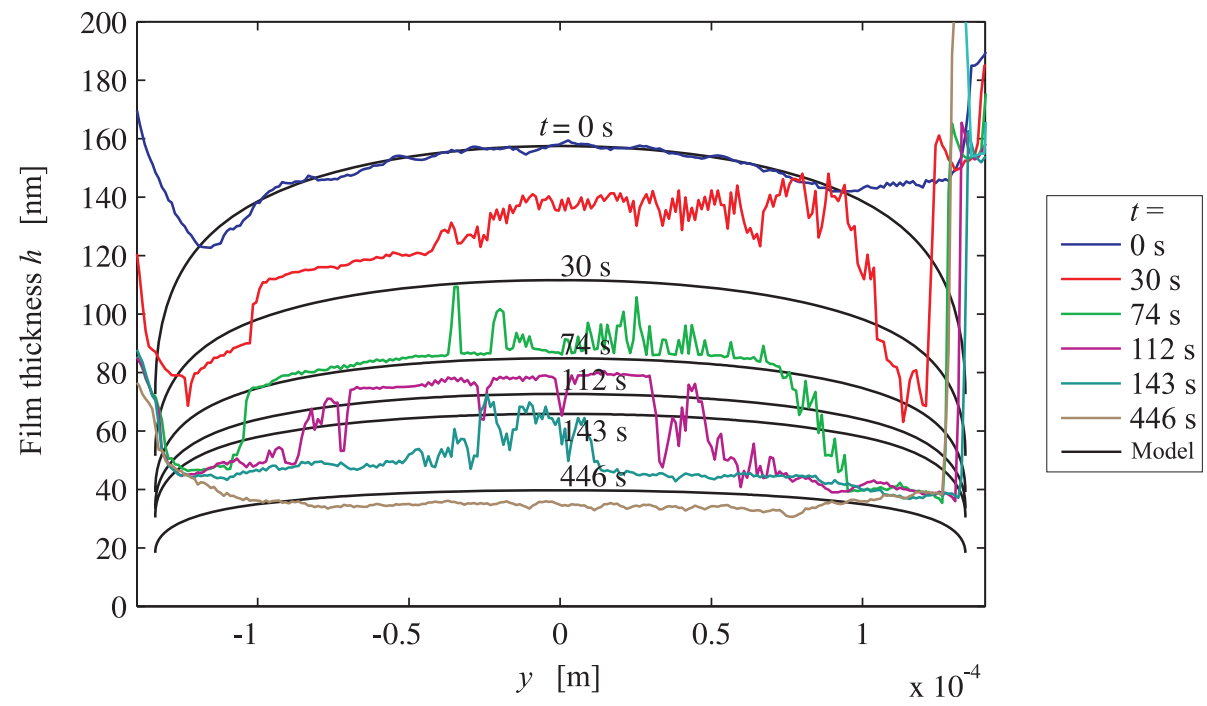

Figure 5.11: Measured (colored lines) and calculated (black lines) film thickness distribution. Parameters: $u_{m}=107 \mathrm{~mm} / \mathrm{s}, \quad F=19 \mathrm{~N}, \quad R_{\text {disk }}=36.5 \mathrm{~mm}, \quad T=22.2^{\circ} \mathrm{C}, \quad \eta_{0}=0.96 \mathrm{~Pa} \cdot \mathrm{s}$, $p_{h}=0.51 \mathrm{GPa}, a=b=134 \mu \mathrm{m}, N=36, L=8.4$ and $h_{c f f}=463 \mathrm{~nm}$.

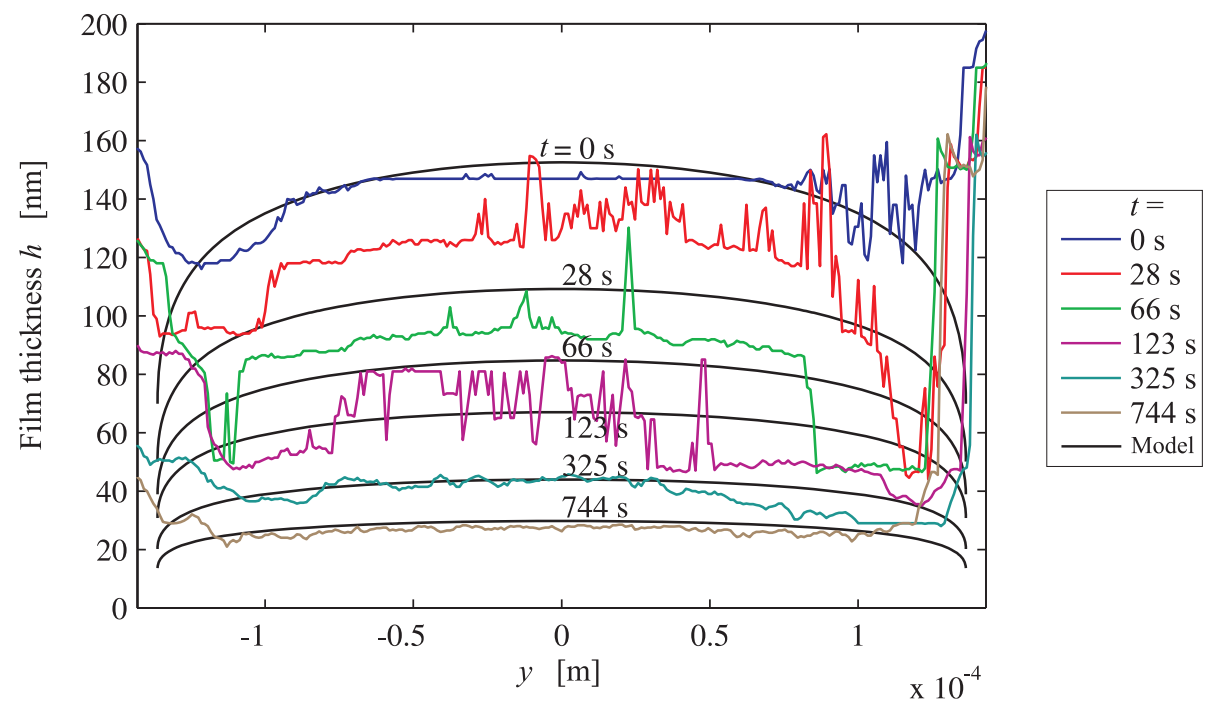

Figure 5.12: Measured (colored lines) and calculated (black lines) film thickness distribution.

Parameters: $u_{m}=107 \mathrm{~mm} / \mathrm{s}, \quad F=20 \mathrm{~N}, \quad R_{\text {disk }}=39.0 \mathrm{~mm}, \quad T=24.7^{\circ} \mathrm{C}, \quad \eta_{0}=0.82 \mathrm{~Pa} \cdot \mathrm{s}$, $p_{h}=0.51 \mathrm{GPa}, a=b=136 \mu \mathrm{m}, N=43, L=8.1$ and $h_{c f f}=418 \mathrm{~nm}$. 


\subsubsection{Central film thickness}

The measurements of the central film thickness were carried out for different velocities and loads for a circular as well as an elliptical contact. The test conditions for the measurement series are given in Table 5.3. The fully flooded film thickness $h_{c f f}$ is calculated numerically.

The experimental results for the circular and the elliptical contact at different velocities and loads are presented in Figure 5.13, Figure 5.14 and Figure 5.15. The measured values are represented by the dots. The solid lines show the predicted film thickness that is calculated by means of Eq. (5.28), i.e. the asymptotic film thickness decay model. The initial layer thickness of the measurement series differs. In order to compare the series, the starting time of each series is chosen such that the first measurement value coincides with the theoretical film thickness.

The measured film thickness of the circular contact (Figure 5.13) is larger than the model predicts. This difference may be explained by reflow effects. That the reflow is considerable is noticeable at the end of the measurement series, where an equilibrium film thickness is reached. At higher velocities the equilibrium is reached for a thicker film, which is most likely caused by extra reflow at higher speeds due to the occurrence of more spin or sideward slip, as was discussed in the preceding section. By reducing the levees at the side of the track before the experiment the replenishment is reduced significantly, but it is difficult to eliminate the reflow effects completely, as the levees on the ball could not be removed and during the measurements new small levees are formed on the disk as well.

The measured film thickness for the elliptical contact (Figure 5.14) is in good agreement with the predicted thickness. Contrary to the circular contact case, the measurements do not show an equilibrium film thickness, which suggests that the reflow effects are smaller here. This may be explained by the larger track width and the smaller gradient of the levees, compared to the circular contact. Also, as the rotation around the spin axis is suppressed by the carriage of the roller, reflow due to spin cannot occur. The model predicts a layer decrease that is independent of the velocity. This is confirmed by the measurements. Notice that at the end of the measurement series the film thickness decay is larger than predicted by the model. As the difference increases at higher speeds and an increasing non-symmetric layer thickness distribution was observed at higher speeds, this is probably caused by centrifugal effects or sideward slip. For more experimental results for the circular and elliptic contact at different speeds, the reader is referred to appendix C.3.

Finally, the asymptotic model predicts a lower layer decay and film thickness decay at higher loads. As mentioned in section 5.2.3 this result was quite unexpected. However, as can be seen in Figure 5.15 this indeed occurs and the physical explanation is that the side flow reduces for higher loads due to a larger viscosity in the central region of the contact. 


\begin{tabular}{|c|c|c|c|c|c|c|c|c|c|c|c|}
\hline Nr. & $\begin{array}{c}u_{m} \\
(\mathrm{~mm} / \mathrm{s})\end{array}$ & $\begin{array}{c}F \\
(\mathrm{~N})\end{array}$ & $\begin{array}{c}p_{h} \\
(\mathrm{GPa})\end{array}$ & $\begin{array}{c}a \\
(\mu \mathrm{m})\end{array}$ & $\begin{array}{c}b \\
(\mu \mathrm{m})\end{array}$ & $\begin{array}{c}T \\
\left({ }^{\circ} \mathrm{C}\right)\end{array}$ & $\begin{array}{c}\eta_{0} \\
(\mathrm{~Pa} . \mathrm{s})\end{array}$ & $\begin{array}{c}R_{\text {disk }} \\
(\mathrm{mm})\end{array}$ & $\begin{array}{c}h_{\text {cff }} \\
(\mathrm{nm})\end{array}$ & $N$ & $L$ \\
\hline 1 & 60 & 20 & 0.51 & 136 & 136 & 24.5 & 0.83 & 36.0 & 295 & 65 & 7.0 \\
\hline 2 & 186 & 20 & 0.51 & 136 & 136 & 24.6 & 0.83 & 36.0 & 625 & 28 & 9.3 \\
\hline 3 & 500 & 20 & 0.51 & 136 & 136 & 25.0 & 0.80 & 35.5 & 1159 & 14 & 12 \\
\hline 4 & 60 & 30 & 0.33 & 108 & 398 & 24.2 & 0.85 & 35.8 & 393 & 36 & 7.0 \\
\hline 5 & 186 & 30 & 0.33 & 108 & 398 & 24.2 & 0.85 & 35.8 & 856 & 15 & 9.3 \\
\hline 6 & 500 & 30 & 0.33 & 108 & 398 & 24.2 & 0.85 & 35.8 & 1618 & 7.3 & 12 \\
\hline 7 & 186 & 10 & 0.23 & 74.5 & 276 & 24.4 & 0.84 & 35.8 & 802 & 5.1 & 9.3 \\
\hline 8 & 186 & 50 & 0.40 & 127 & 472 & 24.5 & 0.83 & 35.8 & 801 & 26 & 9.3 \\
\hline
\end{tabular}

Table 5.3: Test conditions, measurement of the central film thickness, series $1-8$.

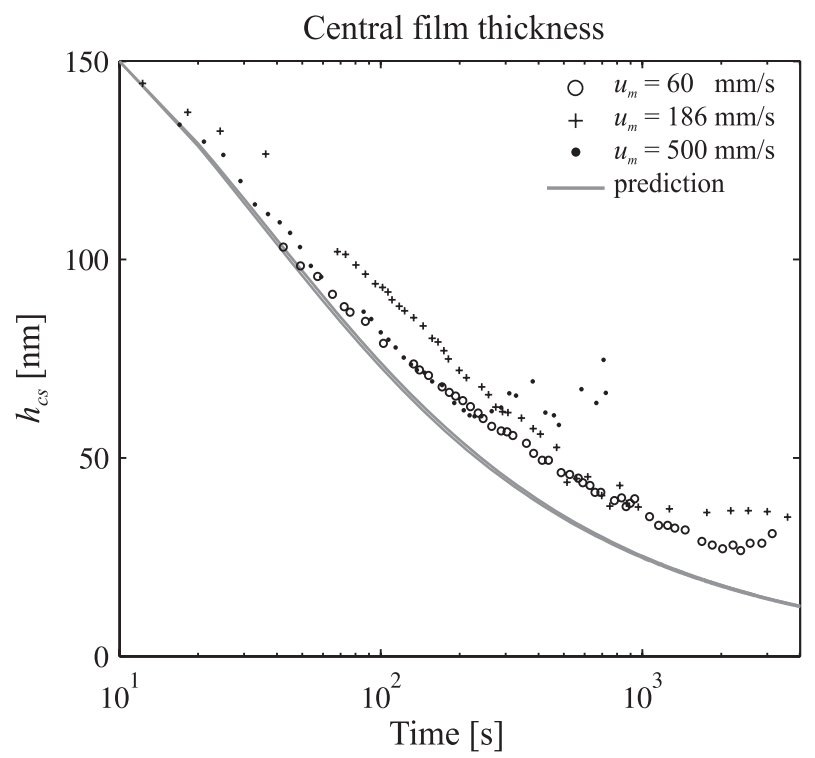

Figure 5.13: Measured and theoretical central film thickness. Measurement series $1-3$ : Circular contact at different velocities, for parameters see Table 5.3. 


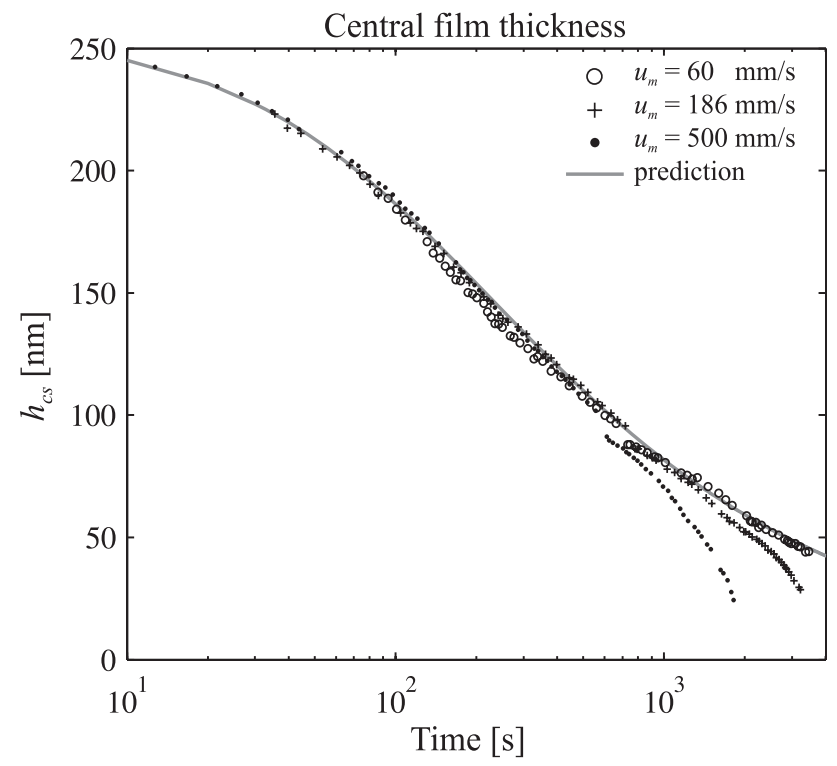

Figure 5.14: Measured and theoretical central film thickness. Measurement series $4-6$ : Elliptical contact at different velocities, for parameters see Table 5.3.

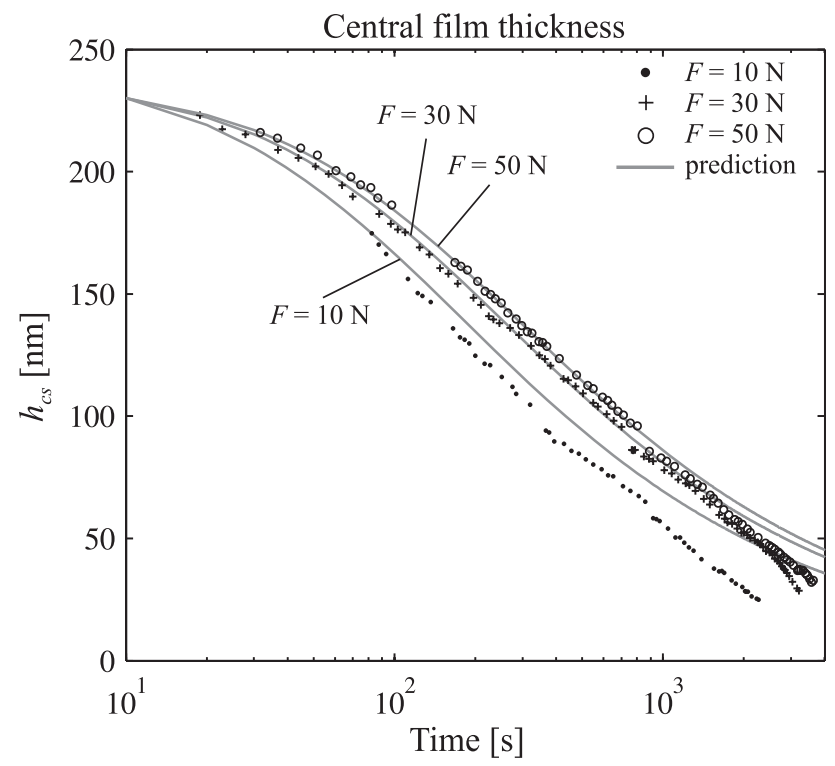

Figure 5.15: Measured and theoretical central film thickness. Measurement series 5, 7 and 8: Elliptical contact at different loads, for parameters see Table 5.3. 


\subsection{Discussion}

\subsubsection{Film thickness decay model Chevalier/Damiens}

Chevalier [16] and Damiens [19] presented a model for the prediction of the decay of the combined thickness of the layers at the inlet to the EHL contact $h_{\text {oil }}$ as a function of the number of overrollings $n$ :

$$
r_{n+1}=\frac{r_{n}}{\sqrt[\gamma]{1+\left(r_{n}\right)^{\gamma}}},
$$

where:

$$
r_{n}=\frac{h_{o i l, n}}{h_{o i l, 1}} .
$$

$h_{\text {oil }, n}$ denotes the combined layer thickness for the $n$-th overrolling and $h_{\text {oil, } 1}$ the combined layer thickness at the outlet of the contact, when the contact is operating under fully flooded conditions and no reflow occurs. The parameter $\gamma$ is derived from a number of numerically calculated solutions of the starved EHL contact problem. The results for $\gamma$ are presented in [19], as a function of the non-dimensional inlet length parameter $\sqrt{M / L}$ and for three values of the ratio $r_{n}$.

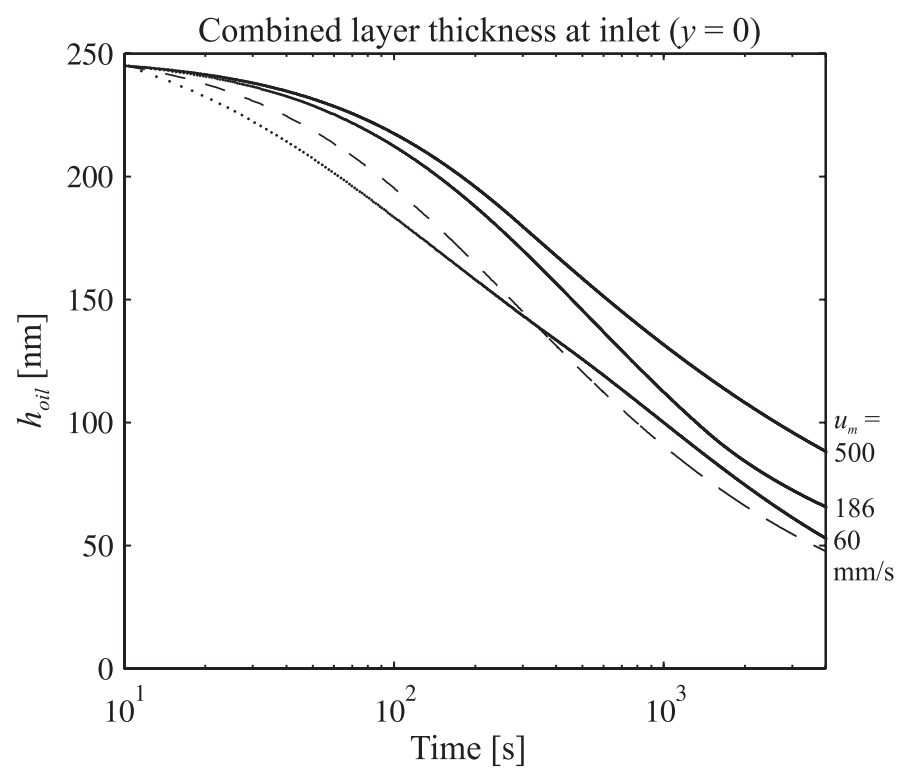

Figure 5.16: The decay of the combined thickness of the supply layers $\left(h_{\text {oil }}\right)$ predicted by Damiens (solid line) and the asymptotic film thickness decay model (dashed line), using the parameters of measurement series 4-6, see Table 5.3. 
For the prediction of the long term film thickness reduction, the film thickness decay model presented in this thesis has two advantages in comparison to the model of Chevalier/Damiens (C/D):

1. The model is a function of time, whereas the $C / D$ film thickness reduction law is a function of the number of overrollings $n$. The number of overrollings is not well defined in a rolling element bearing, as the rollers and the raceways are each overrolled at a different frequency.

2. The ability of the present model to accurately predict the decay rate for small values of $r_{n}=h_{o i l, n} / h_{o i l, 1}$.

The second statement will be illustrated by an example. In Figure 5.16 the layer thickness reduction is shown, as predicted by the $\mathrm{C} / \mathrm{D}$ model, for the conditions of measurement series 4-6. For details regarding the implementation of the C/D model, the reader is referred to appendix C.3. In the figure the layer thickness $h_{\text {oil }}$ for each overrolling is plotted as a dot, however, except for series $4(60 \mathrm{~mm} / \mathrm{s})$, the individual dots are not distinguishable. The dashed line represents the result of the asymptotic film thickness decay model. The reader is reminded that it is a single line as the asymptotic decay model predicts a decay rate that is independent of the velocity, see section 5.2.3. Conversely, the $\mathrm{C} / \mathrm{D}$ model predicts a decay rate that varies with the velocity.

The predictions of the models agree well for low velocities, but differ at higher velocities. This can be explained by the accuracy of determining the value of the parameter $\gamma$. Damiens [19] points out that, for smaller values of $r_{n}$, the accuracy of the presented $\gamma$ values is not excellent, as the small inlet lengths could not be represented well in the computational grid. A second reason why the parameter $\gamma$ may be inaccurate for thinner layers is that the dependence of $\gamma$ on $\sqrt{M / L}$ is based on inlet length consideration only. However, for thinner layers, i.e. smaller $r_{n}$, the influence of the layer reduction at the outlet may no longer be neglected. For that case the dependence of $\gamma$ on $\sqrt{M / L}$ may not be valid. Hence, the presented results for the C/D model for smaller $r_{n}$, i.e. larger $h_{\text {oil, } 1}$, and thus larger velocities are less accurate, which explains the observed deviation between the results of the $\mathrm{C} / \mathrm{D}$ model and the results presented here.

\subsubsection{Centrifugal effects and contact pressure effects}

In this section the relative importance of the contact pressure effect and the centrifugal effect in a bearing is studied. This is done by comparing the rate of mass flow to the side on the track due to these effects. For the centrifugal effects this mass flow rate is defined by Eq. (3.9). The body force for the inner raceway is of the order of magnitude $f_{s} \approx \rho_{0} \Omega_{i r w}^{2} R_{i r w} \sin \left(\alpha^{\prime}\right)$. In this analysis it is assumed that $f_{s}$ is constant over the width of the track and the body forces on the rollers are of the same order of magnitude. For that case Eq. (3.9) becomes:

$$
\hat{q}_{s}(s, t)=\frac{\rho_{0} \tilde{h}_{\infty}^{3}}{3 \eta_{0}} l_{t} \rho_{0} \Omega_{i r w}^{2} R_{i r w} \sin \left(\alpha^{\prime}\right) .
$$


The rate of mass flow to the side due to the contact pressure effects is defined by Eq. (5.18). In dimensional form it reads:

$$
\hat{q}_{y}(y, t)=\frac{2 \rho_{0} n_{c} \tilde{h}_{\infty}^{3} \kappa p_{h}}{3 \eta_{0}} \frac{y}{b} C\left(\frac{y}{b}\right) .
$$

In Figure $5.17 \hat{q}_{s}$ and $\hat{q}_{y}$ are shown as a function of the position $y / b$ on the track. The parameters used are given in the caption. As in this example a large rotational speed $\Omega_{i r w}$ and a large taper angle $\alpha^{\prime}$ are chosen, the mass flow rate due to centrifugal effect is relatively large. However, the figure shows that the flow rate due to contact pressure effects $\hat{q}_{y}$ is still dominant in a large part of the domain. In the central region the mass flow due to the centrifugal effects $\hat{q}_{s}$ is dominant, though the flow rate is relatively small. When the ellipticity ratio $\kappa$ becomes very small, e.g. in tapered roller bearings for which line contact occurs, the centrifugal effect becomes significant in a larger part of the domain. However, in many cases the contact pressure effects will be dominant near the boundaries, expelling lubricant to the sides of the track. Hence, typically the flow rate due to the centrifugal effect is too small to replenish the track from the side. Moreover, due to the centrifugal effects the lubricant from the reservoirs at the side of the track can migrate away from the track. This has a negative influence on the reflow rate.

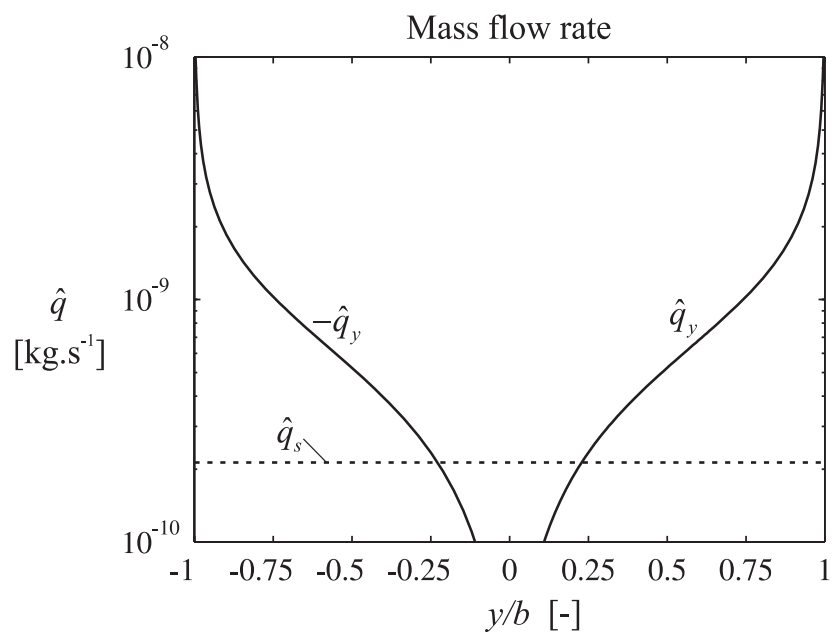

Figure 5.17: Rate of mass flow to the side as a function of the position across the track $y / b$ due to centrifugal effects $\hat{q}_{s}$ and contact pressure effects $\hat{q}_{y}$. Parameters: $n_{c}=16, \kappa=0.01$, $p_{h}=3 \mathrm{GPa}, \quad l_{t}=0.25 \mathrm{~m}, \quad \rho_{0}=850 \mathrm{~kg} / \mathrm{m}^{3}, \quad \Omega_{i r w}=1000 \mathrm{rad} / \mathrm{s}, \quad R_{i r w}=0.05 \mathrm{~m}, \alpha^{\prime}=45^{\circ}$, $\eta_{0}=0.01$ Pa.s, $\alpha=20 \mathrm{GPa}^{-1}$, and $\tilde{h}_{\infty}=100 \mathrm{~nm}$. 


\subsection{Conclusion}

A model has been developed that predicts the change of the supply layer due to contact pressure effects. As the film thickness inside a starved EHL contact is determined by the thickness and distribution of the supply layer, this model can be used to predict the film thickness decay.

The evolution of the layer thickness is obtained from a relatively simple hyperbolic equation. For the thickness of the layer at the center of the track a layer thickness decay formula was derived. Strictly, the EHL starved contact pressure profile and film thickness are required as input. However, for the case of heavily starved contacts, the film thickness decay can be calculated, with adequate approximation, using the Hertzian contact pressure and gap width. In this way, without having to solve the starved EHL problem numerically, a prediction is obtained of the film thickness decay for the long term operation of cylindrical and elliptic contacts under starved conditions.

The model has been validated employing experimental data. It was found that for the circular contact reflow effects are considerable. The measured film thickness of the elliptical contacts is in good agreement with the predicted thickness. The asymptotic model predicts a film thickness decay that is independent of velocity and predicts a smaller central layer thickness decay at higher loads. This was confirmed by the experimental results.

It was shown that the flow rate due to the contact pressure is large compared to the flow rate due to the centrifugal effect. Therefore, the centrifugal effects are too small to replenish the track from the side. 


\section{Chapter 6}

\section{LAYER THICKNESS DECAY IN RADIALLY}

\section{LOADED BEARINGS}

In Chapter 5 the effect of the EHL contact pressure on the thickness of the lubricant layers on the running track was studied. A model was presented for the prediction of layer thickness as a function of time, for the case reflow effects are negligible. This model is applicable for a single contact situation at a constant load, e.g. as in a ball on disk measurement apparatus, or a multiple contact situation, for which the contact geometry and the load are constant and equal for each contact. This for example occurs in purely axially loaded thrust bearings.

However, in many cases a bearing is (also) loaded in radial direction. In that case the load varies over the circumference of the bearing. Furthermore, the geometry of the steel surfaces can be different for the inner and outer raceway. In this chapter the model presented in Chapter 5 is extended to include the effects of the load variation and the difference in the geometry of the outer and inner raceway. Results of the layer thickness as a function of time will be presented for a ball bearing and for a spherical roller bearing. In both cases the effects of the bearing load and the rotational speed on the rate of decay of the layer thickness are investigated.

\subsection{Theoretical formulation}

The first step is to quantify the mass flow rate in the EHL contacts perpendicular to the rolling direction, i.e. the side flow. Next, this flow rate is used to determine the decrease of the supply layer thickness on the track. The side flow in an EHL contact depends on the load, see Chapter 5. In this study the applied load to the EHL contacts varies along the circumference of the bearing. First, it will be shown how the load distribution in the bearing is defined. Subsequently, the equations for the side flow rate are derived.

\subsubsection{Load distribution}

According to Harris [36] in most cases the load distribution in a rolling element bearing may be approximated by the load distribution in a statically loaded bearing. Subsequently, in order to obtain the dynamic load distribution, the centrifugal force acting on the rollers can be added to the static load distribution on the outer raceway. For a bearing under pure radial load $F_{r}$ a schematic representation of the different contributions to the load distribution is shown in Figure 6.1. 


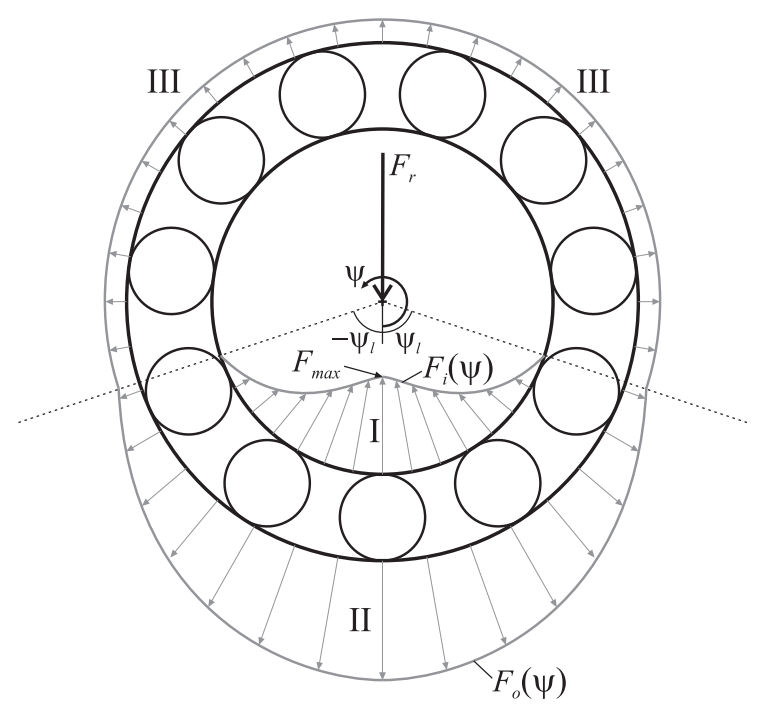

Figure 6.1: Schematic representation of the load distribution on the inner raceway $F_{i}(\psi)$ and the outer raceway $F_{o}(\psi)$ for a bearing under pure radial load $F_{r}$. Loaded zones: Inner raceway (I), Outer raceway (II, III).

$\psi$ denotes the angular position along the circumference of the bearing. Depending on $\psi$, three regions can be distinguished in which different contributions act:

I: $\quad$ Static load distribution on the inner raceway. $\psi \in\left[-\psi_{l}, \psi_{l}\right]$

II: $\quad$ Static load distribution and centrifugal force on the outer raceway. $\psi \in\left[-\psi_{l}, \psi_{l}\right]$

III: Centrifugal force on the outer raceway. $\psi \in\left[\psi_{l}, 2 \pi-\psi_{l}\right]$

The static load distribution, i.e. the load distribution on the inner raceway, for a rigidly supported bearing subjected to a radial load $F_{r}$, is given by Harris [36]:

$$
F_{i}(\psi)=F_{\max }\left(1-\frac{1}{2 \varepsilon}(1-\cos (\psi))\right)^{n},
$$

where $\varepsilon$ is the load distribution factor determining the size of the load zones I and II, i.e. $\psi_{l}$. The power $n$ is 1.5 for circular and elliptical contacts and $n$ is 1.11 for line contacts. $F_{\max }$ is the maximum static load:

$$
F_{\max }=K_{n}\left(\frac{P_{d} \varepsilon}{1-2 \varepsilon}\right)^{n},
$$




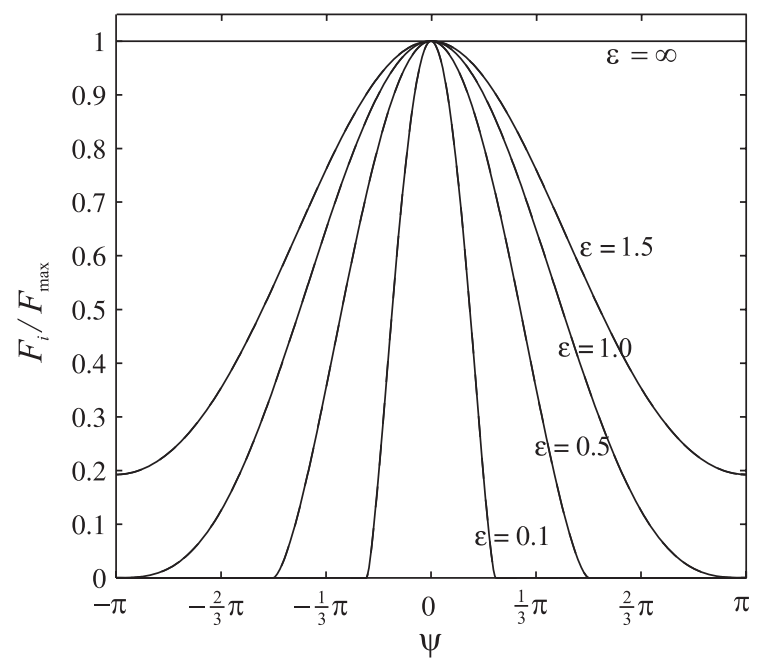

Figure 6.2: Dimensionless load distribution for various values of the load distribution factor $\varepsilon$.

$P_{d}$ is the diametral clearance, which for normal bearing operation is positive, although improper mounting of the bearing or large temperature differences between the inner and the outer ring can result in a negative clearance. $K_{n}$ is the load deflection factor, which is a combination of the deflection factors of the contacts on the inner raceway $\left(K_{i}\right)$ and the ones on the outer raceway $\left(K_{o}\right)$ according to:

$$
K_{n}=\left(K_{i}^{-1 / n}+K_{o}^{-1 / n}\right)^{-n}
$$

For a circular or elliptical contact $K_{i}$ and $K_{o}$ are defined by (see Eq. (2.13) in [71]):

$$
K_{i / o}=\frac{2}{3} E^{\prime} \sqrt{2 R \pi^{2} \mathcal{E} /\left(4 \kappa^{2} \mathcal{K}^{3}\right)} .
$$

For the definition of the variables in Eq. (6.4), the reader is referred to Chapter 4 and the nomenclature. The load distribution factor $\varepsilon$ is a function of the radial load $F_{r}$ and the number of rollers $n_{r}$, and can be solved from the static load equilibrium:

$$
F_{r}=\frac{n_{r}}{2 \pi} \int_{-\psi_{l}}^{\psi_{1}} F_{i}(\psi) \cos (\psi) d \psi,
$$

where $\psi_{l}$ is the angular location of the boundary of the load zones, see Figure 6.1:

$$
\psi_{l}=\left\{\begin{array}{ll}
\operatorname{acos}(1-2 \varepsilon) & 0 \leq \varepsilon<1 \\
\pi & \varepsilon \geq 1
\end{array} .\right.
$$




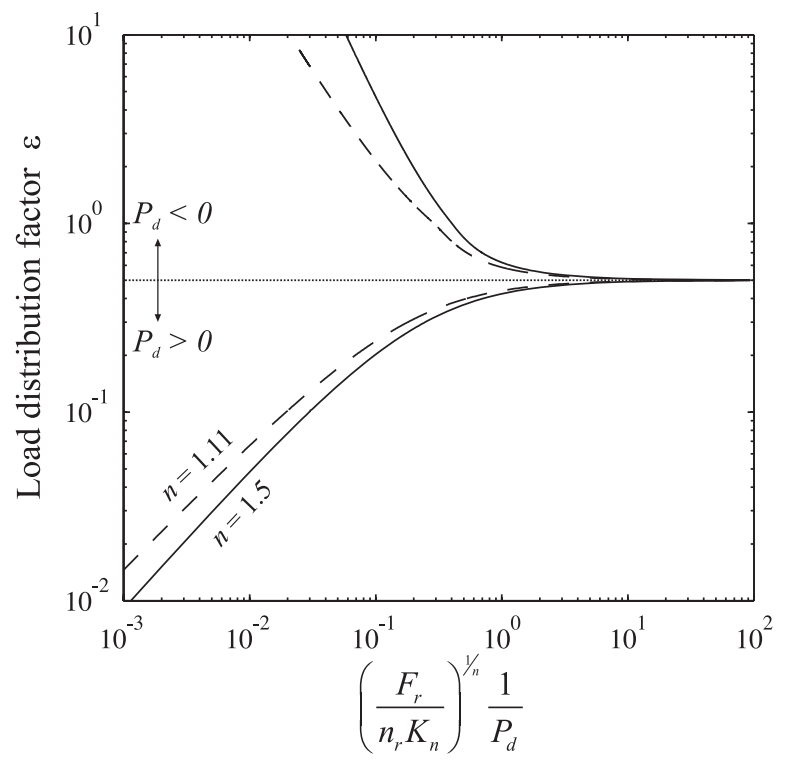

Figure 6.3: The load distribution factor $\varepsilon$ as a function of the radial load $F_{r}$, the number of rollers $n_{r}$, the load deflection factor $K_{n}$, and the diametral clearance $P_{d}$.

In Figure 6.2 the load distribution is shown for various values of the load distribution factor $\varepsilon$. The distribution is scaled by the maximum static load $F_{\max }$ occurring at $\psi=0$. For $\varepsilon>1$ the static load distribution is nonzero over the entire circumference and in the limit for $\varepsilon \rightarrow \infty$ the load distribution becomes uniform. However, values of $\varepsilon>0.5$ only occur for negative diametral clearances, which is uncommon. This is shown in Figure 6.3, where the load distribution factor $\varepsilon$ is given as a function of the radial load $F_{r}$, the number of rollers $n_{r}$ the load deflection factor $K_{n}$, and the diametral clearance $P_{d}$. The solution of $\varepsilon$ is obtained by solving Eq. (6.5), with Eqs. (6.1), (6.2), and (6.6). The figure shows that for a positive diametral clearance, $\varepsilon$ increases with increasing $F_{r}$ and decreasing $n_{r}, K_{n}$, and $P_{d}$.

The centrifugal force that acts on the outer contact of a roller is proportional to the mass of the roller $m_{r}$ times its acceleration:

$$
F_{c}=m_{r} \Omega_{c a}^{2}\left(R_{x, r o l}+R_{x, i r w}\right),
$$

where $\Omega_{c a}$ the rotational speed of the cage and $R_{x, \text { rol }}$ and $R_{x, \text { irw }}$ are the radii of the rolling element and of the inner raceway, respectively. Finally, the load distribution on the outer raceway is obtained by adding the centrifugal force $F_{c}$ to the static load distribution:

$$
F_{o}(\psi)=F_{i}(\psi)+F_{c} .
$$




\subsubsection{Mass flow to the side}

The layer thickness distribution in time $\tilde{h}_{\infty}(y, t)$ can be obtained from the continuity equation (5.1). Input to this equation is the rate of mass flow to the side integrated over the track $\hat{q}_{y}(y, t)$. In the model presented in this chapter this flow rate is only determined by the mass flow rate in the EHL contacts. Hence, other effects such as the centrifugal effects or reflow effects are not included here. Due to the varying load in the bearing the flow rate in an EHL contact is a function of the angular position. In addition, due to the difference in the geometry of the inner and the outer raceway, it is different for the contacts on the inner raceway and on the outer raceway. According to Eq. (5.2) the mass flow rate $\hat{q}_{y}$ is equal to the sum of the contributions of each EHL contact. When $\hat{q}_{y, i}$ and $\hat{q}_{y, o}$ are the rate of mass flow to the side in an EHL contact on the inner and the outer raceway, respectively, Eq. (5.2) can be written as:

$$
\hat{q}_{y}(y, t)=\sum_{j=1}^{n_{r}}\left(\hat{q}_{y, i}\left(y, t, \psi_{j}\right)+\hat{q}_{y, o}\left(y, t, \psi_{j}\right)\right),
$$

where $n_{r}$ is the total number of rolling elements per row. The index $j$ is used to number the rolling elements. The angular position of rolling element $j$ is defined by:

$$
\psi_{j}=\Omega_{c a} t+\frac{2 \pi j}{n_{r}},
$$

where $\Omega_{c a}$ is the rotational speed of the cage. The time it takes for the rolling elements to exchange their angular position is:

$$
\lambda_{r r}=2 \pi /\left(n_{r} \Omega_{c a}\right) .
$$

Due to the varying load in the bearing the mass flow rate $\hat{q}_{y}$ varies periodically with a wavelength $\lambda_{r r}$. Typically, $\lambda_{r r}$ is very small compared to the time scale considered for the layer evolution. In order to predict the long term behavior of the layer thickness, it is therefore sufficient to replace this high frequent time dependent effect by its average value. Hence, Eq. (6.9) becomes:

$$
\hat{q}_{y}(y, t)=\sum_{j=1}^{n_{r}}\left(\frac{1}{\lambda_{r r}} \int_{0}^{\lambda_{r r}}\left(\hat{q}_{y, i}\left(y, t, \psi_{j}\right)+\hat{q}_{y, o}\left(y, t, \psi_{j}\right)\right) d t\right) .
$$

Substitution of Eq. (6.11) and $d t=d \psi_{j} / \Omega_{c a}$ into Eq. (6.12) and changing integration variables from $t$ to $\psi_{j}=\left[0, \Omega_{c a} \lambda_{r r}\right]=\left[0,2 \pi / n_{r}\right]$ gives:

$$
\begin{aligned}
\hat{q}_{y}(y, t) & =\sum_{j=1}^{n_{r}}\left(\frac{n_{r}}{2 \pi} \int_{0}^{2 \pi / n_{r}}\left(\hat{q}_{y, i}\left(y, t, \psi_{j}\right)+\hat{q}_{y, o}\left(y, t, \psi_{j}\right)\right) d \psi_{j}\right) \\
& =\frac{n_{r}}{2 \pi} \int_{0}^{2 \pi}\left(\hat{q}_{y, i}(y, t, \psi)+\hat{q}_{y, o}(y, t, \psi)\right) d \psi .
\end{aligned}
$$


When the film thickness $h=h(x, y, \psi, t)$ and pressure $p=p(x, y, \psi, t)$ are known for the inner and outer raceway contacts, the flow rates $\hat{q}_{y, i}(y, t, \psi)$ and $\hat{q}_{y, o}(y, t, \psi)$ can be calculated by means of Eq. (5.4). How to obtain the pressure and film thickness is explained next.

\subsubsection{Film thickness and pressure}

As in Chapter 5 it is assumed that due to the equipartition in the contacts for the long term operation, the layer thickness is uniform in circumferential direction and equal for the rollers and the raceways. An example of such a layer thickness distribution for a ball bearing is shown in Figure 6.4. It is assumed that the equipartition of the layers takes place over the full width of the track, even though because of the radial loading the Hertzian contact width may vary along the circumference of the bearing. For ball bearings, this type of distribution only occurs when the load is purely radial. Otherwise, due to the non-zero contact angle between the balls and the raceways, spin motion will occur, which will continuously alter the location of the track on the balls. Under the conditions assumed, the sum of the thickness of the layers close to the inlet $h_{\text {oil }}(y, t)$ for each contact may be approximated as twice the average free surface layer thickness $h_{\infty}(y, t)$.

In spite of the enormously increased efficiency of numerical solution algorithms for starved EHL problems, accurate computation of the film thickness $h$ and the pressure distribution $p$ as a function of both time $t$ and the circumferential direction $\psi$ for long operation times is not feasible in short computing times. In Chapter 5 it was shown that for severely starved conditions and uniform supply layers the contact size and the pressure distribution can be accurately approximated by the Hertzian dry contact solution. The same approximation will be used in this chapter. For severely starved conditions the film

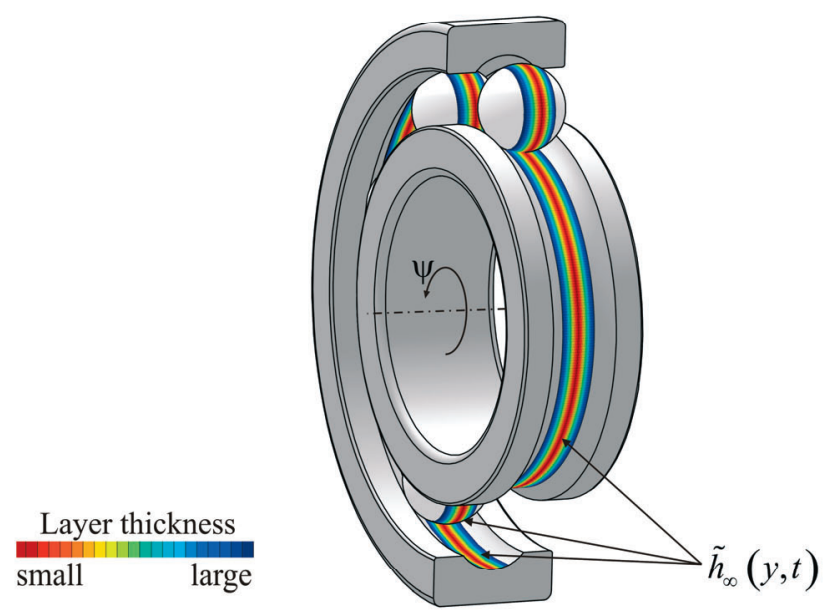

Figure 6.4: Schematic representation of a deep groove ball bearing operating in the starved regime, illustrating that for the long term operation the supply layer thickness distribution and thus the inlet condition $h_{o i l}$ is similar for each contact, under the condition that spin of the balls does not occur. 
thickness for the inner and the outer raceway contacts is defined by Eq. (5.25), which in dimensional variables reads:

$$
h(x, y, \psi, t)=\frac{\rho_{0} h_{\text {oil }}(y, t)}{\rho(p)}=\frac{2 \rho_{0} \tilde{h}_{\infty}(y, t)}{\rho(p)} .
$$

The size of a Hertzian contact, defined by $a$ and $b$, and the central Hertzian pressure $p_{h}$ are a function of the load according to $F^{\frac{1}{3}}$, see Eqs. (4.1), (4.2) and (4.10). Thus, when the load varies along the circumference of the bearing, $a, b$ and $p_{h}$ also vary along the circumference, i.e. with $\psi$, according to:

$$
a(\psi)=\breve{a}\left(\frac{F(\psi)}{F_{\max }}\right)^{\frac{1}{3}}, \quad b(\psi)=\breve{b}\left(\frac{F(\psi)}{F_{\max }}\right)^{\frac{1}{3}}, \quad p_{h}(\psi)=\breve{p}_{h}\left(\frac{F(\psi)}{F_{\max }}\right)^{\frac{1}{3}},
$$

where $\breve{p}_{h}$, $\breve{a}$ and $\breve{b}$ are the Hertzian pressure, the semi-minor axis, and the semi-major axis, respectively, of a Hertzian contact loaded with $F=F_{\max }$. The Hertzian pressure distribution as a function of $\psi$ is obtained by substitution of Eq. (6.15) in Eq. (4.9) and rearranging:

$$
p(x, y, \psi)=\breve{p}_{h} \sqrt{\left(\frac{F(\psi)}{F_{\max }}\right)^{\frac{2}{3}}-\left(\frac{x}{\breve{a}}\right)^{2}-\left(\frac{y}{\breve{b}}\right)^{2}} .
$$

The location of the boundary of the pressurized region is (see Figure 5.2):

$$
a^{+}(y, \psi)=-a^{-}(y, \psi)=\breve{a} \sqrt{\left(\frac{F(\psi)}{F_{\max }}\right)^{\frac{2}{3}}-\left(\frac{y}{\breve{b}}\right)^{2}} .
$$

The geometry of the steel surfaces and the load distribution is different for the inner and the outer raceway. Therefore, the contact size and pressures are different as well. In the equations in the following sections the indices $i$ and $o$ will be added to the parameters and variables for the inner raceway contacts and the outer raceway contacts, respectively.

\subsubsection{Scaling}

The same scaling is used as in Chapter 5, see Eq. (5.7), with $p_{h}=\breve{p}_{h, i}, a=\breve{a}_{i}, b=\breve{b}_{i}$, and $c=\breve{c}_{i}$, and the scaling of $\hat{q}_{y, i}$ and $\hat{q}_{y, o}$ is similar to the scaling of $\hat{q}_{y, k}$. The following ratios are defined:

$$
\phi_{a}=\breve{a}_{i} / \breve{a}_{o}, \quad \phi_{b}=\breve{b}_{i} / \breve{b}_{o} .
$$

The load is scaled according to:

$$
\bar{F}_{i}(\psi)=\frac{F_{i}(\psi)}{F_{\max }}, \quad \bar{F}_{o}(\psi)=\frac{F_{o}(\psi)}{F_{\max }}, \quad \bar{F}_{c}=\frac{F_{c}}{F_{\max }} .
$$


Substitution of Eq. (6.1) and Eq. (6.8) into Eq. (6.19) gives the dimensionless load distribution for the inner and the outer raceway, respectively:

$$
\begin{gathered}
\bar{F}_{i}(\psi)=\left(1-\frac{1}{2 \varepsilon}(1-\cos (\psi))\right)^{n}, \\
\bar{F}_{o}(\psi)=\bar{F}_{o}(\psi)+\bar{F}_{c} .
\end{gathered}
$$

$\hat{Q}_{Y}$ is obtained using Eqs. (5.10), (6.13), (6.16), (6.17) and (6.14) in scaled variables. For the derivation of $\hat{Q}_{Y}$ the reader is referred to Appendix D.1. It can be written in the same form as in Chapter 5:

$$
\hat{Q}(Y)=Y C(Y),
$$

where the function $C(Y)$ is now defined by:

$$
C(Y)=\frac{1}{4 \pi} \int_{0}^{2 \pi}\left(\int_{-\pi / 2}^{\pi / 2}\left(\left(\bar{\rho}\left(P_{i}\right)\right)^{-2}\left(\bar{\eta}\left(P_{i}\right)\right)^{-1}\right) d \varphi+\phi_{b}^{3} \int_{-\pi / 2}^{\pi / 2}\left(\left(\bar{\rho}\left(P_{o}\right)\right)^{-2}\left(\bar{\eta}\left(P_{o}\right)\right)^{-1}\right) d \varphi\right) d \psi
$$

The expressions for the dimensionless density $\bar{\rho}(P)$ and viscosity $\bar{\eta}(P)$ are Eq. (4.25) and Eq. (4.27), respectively. The equations for the dimensionless pressure for the inner and outer raceway contacts are:

$$
\begin{aligned}
& P_{i}(\varphi, Y, \psi)=\sqrt{\left(\bar{F}_{i}(\psi)\right)^{\frac{2}{3}}-Y^{2}} \cos (\varphi), \\
& P_{o}(\varphi, Y, \psi)=\phi_{a} \phi_{b} \sqrt{\left(\bar{F}_{o}(\psi)\right)^{\frac{2}{3}}-\left(\phi_{b} Y\right)^{2}} \cos (\varphi) .
\end{aligned}
$$

\subsubsection{Layer thickness distribution}

The equations for the dimensionless layer thickness $\tilde{H}(Y, \bar{t})$ presented in section 5.1.4 are also applicable here. Input to these equations is the function $C(Y)$, which is now defined by Eq. (6.23). Analysis of the system of dimensional equations shows that the dimensionless layer thickness $\tilde{H}(Y, \bar{t})$, depends only on the contact size ratios $\phi_{a}$ and $\phi_{b}$, the load distribution parameters $\varepsilon, n$ and $\bar{F}_{c}$, and the parameters of the viscosity-pressure and the density-pressure relation $\alpha, \eta_{0}$ and $p_{h}=\breve{p}_{h, i}$.

To obtain $C(Y)$ the integration with respect to $\psi$ in Eq. (6.23) is discretized using $10^{6}$ uniformly distributed nodes. A recursive adaptive Simpson quadrature approximation to the inner integrals of Eq. (6.23) was used with an absolute error tolerance of $10^{-6}$. The calculation time for $C(Y)$ is dramatically reduced by pre-computation of inner integrals for a limited number of uniformly distributed values of the parameter group in front of the cosines of Eq. (6.24). It was found that 100 pre-calculated values are sufficient. An approximation of the inner integrals as a function of this parameter group is constructed by linear interpolation between the pre-computed values. 


\subsubsection{Central layer thickness}

The solution for the dimensionless layer thickness at $Y=0$ is defined by Eq. (5.24). In dimensional form it reads:

$$
\tilde{h}_{\infty}(0, t)=\left(C_{3} t+\tilde{h}_{\infty, 0}^{-2}\right)^{-\frac{1}{2}},
$$

where $\tilde{h}_{\infty, 0}$ is the initial layer thickness and:

$$
C_{3}=\frac{2 C}{\tau c^{2}}=\frac{4 n_{c} p_{h} a C}{3 \eta_{0} l_{t} b^{2}}=\frac{n_{c} E^{\prime} \kappa^{2} C}{3 \eta_{0} l_{t} R \mathcal{E}} .
$$

The parameters $E^{\prime}, \kappa, R$, and $\mathcal{E}$ are related to the inner raceway contacts and $p_{h}=\breve{p}_{h, i}$, $a=\breve{a}_{i}, b=\breve{b}_{i}$, and $c=\breve{c}_{i}$. The parameter $C=C(0)$ is defined by Eq. (6.23).

\subsection{Results and discussion}

Results of the film thickness as a function of time will be presented for two bearings: a radial ball bearing (209) and a spherical roller bearing (22317). For the properties of these bearings the reader is referred to appendix D.3. In the examples the dynamic viscosity is $\eta_{0}=0.02 \mathrm{~Pa}$.s and the pressure-viscosity coefficient is $\alpha=20 \cdot 10^{-9} \mathrm{~Pa}^{-1}$. In the following, the decay of the central layer thickness with time is discussed. Subsequently, the results for the layer thickness distribution across the track are presented.

\subsubsection{Central layer thickness}

The spherical roller bearing is considered for the case of a bearing load of $20 \mathrm{kN}$ and a rotational velocity of $3000 \mathrm{rpm}$. This bearing consists of two rows of rollers that are positioned at a small angle relative to the axis of rotation of the bearing. The effect of this angle on the load distribution is neglected. Hence, the load on each row is assumed to be $F_{r}=10 \mathrm{kN}$. For this loading, the maximum predicted Hertzian contact width $b$ is $17.6 \mathrm{~mm}$, which is smaller than the width of the roller itself $(20.7 \mathrm{~mm})$. Therefore, according to Harris [36], the Hertzian contact area for each contact will be elliptic.

Suppose that $t_{c r}$ is the period after which the layer thickness is reduced to some critical value $\tilde{h}_{c r}$. This period can be found by rewriting Eq. (6.25):

$$
t_{c r}=t_{c r}\left(\tilde{h}_{c r}, \tilde{h}_{\infty, 0}\right)=\frac{1}{C_{3}}\left(\frac{1}{\tilde{h}_{c r}^{2}}-\frac{1}{\tilde{h}_{\infty, 0}^{2}}\right) .
$$

When the initial layer thickness is $\tilde{h}_{\infty, 0}=50 \mathrm{~nm}$ and the critical layer thickness is chosen equal to $\tilde{h}_{c r}=10 \mathrm{~nm}$ the critical time becomes:

$$
t_{c r}=\frac{9.6 \cdot 10^{15} \mathrm{~m}^{2}}{C_{3}}
$$




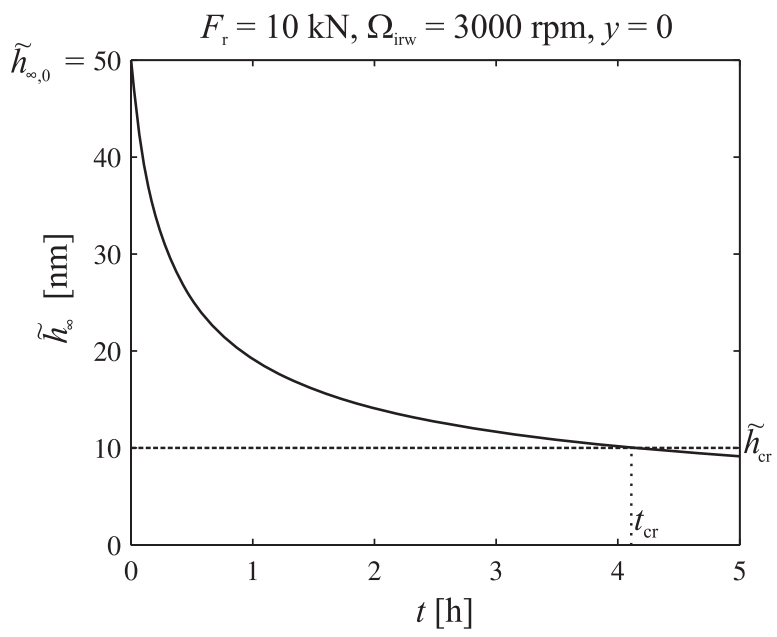

Figure 6.5: Predicted central layer thickness as a function of time for spherical roller bearing 22317.

In Figure 6.5 the predicted central layer thickness is shown as a function of time for the spherical roller bearing. Also, the critical time $t_{c r}$ is shown, which for this case is 4.1 hour.

Next, the effects of the bearing load and rotational velocity on the decay rate will be discussed. As a reference for the decay rate the critical time $t_{c r}$ defined by Eq. (6.28) is taken. In Table 6.1 the predicted times $t_{c r}$ are given for the spherical roller bearing and the radial ball bearing for different loads and rotational velocities. From these results, it can be seen that the predicted times $t_{c r}$ are considerably larger for the spherical roller bearing than for the ball bearing. This can be explained by the difference in the contact geometry parameters $\kappa$ and $R$. According to Eq. (6.28), $t_{c r}$ is inversely proportional to $C_{3}$, which itself is linearly dependent on the ratio $\kappa^{2} / R$, see Eq. (6.26). This ratio is 38 times larger for the ball bearing than for the spherical roller bearing. As the difference of the other parameters involved is considerably smaller or negligible, the difference in decay time stems from this ratio.

Furthermore, the results in Table 6.1 show that at a constant angular velocity $\Omega_{\text {irw }}$ a change of the bearing load $F_{r}$ does not significantly alter the results for $t_{c r}$. However, a change of the angular velocity $\Omega_{i r w}$ has a relatively large effect. This can be explained by the effect of the centrifugal force on the side flow rate. The centrifugal force $F_{c}$ is considerably smaller than the forces due to the static loading, see $F_{\max }$ in Table 6.1. Due to the exponential viscosity-pressure dependence, larger loads, i.e. larger pressures, result in larger viscosities and a reduced side flow. This effect is shown in the theoretical and experimental results presented in Chapter 5. Consequently, in the bearing the side flow due to the centrifugal force (zone III in Figure 6.1) is considerably larger than the side flow in the highly loaded zone (I en II). As a result the layer thickness decay period $t_{c r}$ is mainly determined by the flow in zone III, which is determined by the centrifugal force and thus the velocity. 


\section{Spherical roller bearing 22317}

\begin{tabular}{|c|c|c|c|c|c|c|c|}
\hline & Nr. & 1 & 2 & 3 & 4 & 5 & \\
\hline Radial load & $F_{r}$ & 10 & 10 & 10 & 5 & 2.5 & $\mathrm{kN}$ \\
\hline Rotational speed inner raceway & $\Omega_{i r w}$ & 750 & 1500 & 3000 & 3000 & 3000 & rpm \\
\hline Contact speed & $u_{m}$ & 2.56 & 5.11 & 10.2 & 10.2 & 10.2 & $\mathrm{~m} / \mathrm{s}$ \\
\hline Centrifugal load on roller & $F_{c}$ & 5.52 & 22.1 & 88.3 & 88.3 & 88.3 & $\mathrm{~N}$ \\
\hline Maximum static load & $F_{\max }$ & 4.98 & 4.98 & 4.98 & 2.82 & 1.62 & $\mathrm{kN}$ \\
\hline Load distribution factor & $\varepsilon$ & 0.16 & 0.16 & 0.16 & 0.12 & 0.09 & - \\
\hline $\begin{array}{l}\text { Maximum Hertzian pressure } \\
\text { inner raceway contact, with } \\
F=F_{\text {max }}\end{array}$ & $\breve{p}_{h, i}$ & 1.23 & 1.23 & 1.23 & 1.02 & 0.85 & $\mathrm{GPa}$ \\
\hline $\begin{array}{l}\text { Half length inner raceway } \\
\text { contact, with } F=F_{\max }\end{array}$ & $\breve{a}_{i}$ & 0.220 & 0.220 & 0.220 & 0.182 & 0.151 & $\mathrm{~mm}$ \\
\hline $\begin{array}{l}\text { Half width inner raceway } \\
\text { contact, with } F=F_{\max }\end{array}$ & $\breve{b}_{i}$ & 8.80 & 8.80 & 8.80 & 7.29 & 6.06 & $\mathrm{~mm}$ \\
\hline $\begin{array}{l}\text { Fully flooded central film } \\
\text { thickness inner raceway } \psi=0\end{array}$ & $h_{c f f}$ & 122 & 194 & 309 & 321 & 333 & $\mathrm{~nm}$ \\
\hline Decay time & $t_{c r}$ & 1.8 & 2.7 & 4.1 & 4.0 & 3.8 & $\mathrm{~h}$ \\
\hline \multicolumn{8}{|l|}{ Deep groove ball bearing 209} \\
\hline & Nr. & 6 & 7 & 8 & 9 & 10 & \\
\hline Radial load & $F_{r}$ & 10 & 10 & 10 & 5 & 2.5 & $\mathrm{kN}$ \\
\hline Rotational speed inner raceway & $\Omega_{i r w}$ & 1500 & 3000 & 6000 & 6000 & 6000 & rpm \\
\hline Contact speed & $u_{m}$ & 2.46 & 4.91 & 9.82 & 9.82 & 9.82 & $\mathrm{~m} / \mathrm{s}$ \\
\hline Centrifugal load on roller & $F_{c}$ & 0.27 & 1.09 & 4.34 & 4.34 & 4.34 & $\mathrm{~N}$ \\
\hline Maximum static load & $F_{\max }$ & 5.07 & 5.07 & 5.07 & 2.59 & 1.34 & $\mathrm{kN}$ \\
\hline Load distribution factor & $\varepsilon$ & 0.44 & 0.44 & 0.44 & 0.41 & 0.38 & - \\
\hline $\begin{array}{l}\text { Maximum Hertzian pressure } \\
\text { inner raceway contact, with } \\
F=F_{\text {max }}\end{array}$ & $\breve{p}_{h, i}$ & 3.21 & 3.21 & 3.21 & 2.57 & 2.06 & $\mathrm{GPa}$ \\
\hline $\begin{array}{l}\text { Half length inner raceway } \\
\text { contact, with } F=F_{\max }\end{array}$ & $\breve{a}_{i}$ & 0.285 & 0.285 & 0.285 & 0.228 & 0.183 & $\mathrm{~mm}$ \\
\hline $\begin{array}{l}\text { Half width inner raceway } \\
\text { contact, with } F=F_{\max }\end{array}$ & $\breve{b}_{i}$ & 2.64 & 2.64 & 2.64 & 2.12 & 1.70 & $\mathrm{~mm}$ \\
\hline $\begin{array}{l}\text { Fully flooded central film } \\
\text { thickness inner raceway } \psi=0\end{array}$ & $h_{c f f}$ & 92 & 147 & 234 & 245 & 256 & $\mathrm{~nm}$ \\
\hline Decay time & $t_{c r}$ & 0.057 & 0.086 & 0.127 & 0.118 & 0.110 & $\mathrm{~h}$ \\
\hline
\end{tabular}

Table 6.1: Parameters and central layer thickness decay times for the single row deep-groove ball bearing 209 and the spherical roller bearing 22317. 
Finally, it is found that the predicted times $t_{c r}$ in Table 6.1 are very short compared to the observed grease life in actual bearings, which is generally of the order of thousands of hours. The assumed critical layer thickness $\tilde{h}_{c r}$ may be smaller in practice, which will give longer times $t_{c r}$. However, this cannot explain the large difference between the predicted critical times and the grease life. As the single contact behavior predicted by the model was found to be very accurate, the difference in critical times must be explained by a considerable amount of replenishment of the track taking place in a bearing, which on average counteracts the strong tendency of the EHL contacts to expel lubricant from the track.

The lubricant available for replenishment is situated on the sides of the track and on the cage. In section 5.5.2 it is shown that the flow rate due to the centrifugal effects is too small to replenish the track from the side. Also, the surface tension driven replenishment of the track is very small, see Jacod et al. [40] and Gershuni et al. [32]. Jacod et al. [40] showed that local significant reflow from the sides to the contact may occur due to capillary forces. For ball bearings the spin of the balls is an important supply mechanism. This was shown in the measurement results presented in Chapter 5. The results for the ball-disk contact showed increased film thickness values compared to the model predictions, which was explained by the supply of oil from the side of the track, probably caused by a small amount of spin of the ball. Furthermore, in grease lubricated bearings the supply of base oil from the grease reservoirs on the cage is important. Damiens et al. [21] studied the influence of the cage on the film thickness experimentally, using a ball and disk apparatus. They found that for grease lubrication the cage has a significant effect on the film thickness. This is explained by the redistribution of lubricant in the cage/ball contacts.

Through analysis of the measured temperature of grease lubricated bearings Lugt et al. [50] showed that grease lubrication is not a continuous process, but is characterized by "events" caused by film breakdown and recovery. This means that occasionally there is a considerable supply of lubricant to the track, which is large enough to recover the film. They concluded that grease lubrication exhibits deterministic chaotic behavior and highly depends on the initial filling conditions. Also, the temperature for a cylindrical roller bearing rotating at $6000 \mathrm{rpm}$, presented in [50], show that the time between recovery of the film is of the order 10 hours to 100 hours. This is much shorter than the bearing life. Hence, the incidental recovery of the film is vital for the durability of a bearing, and it is therefore essential to include this effect in a lifetime prediction model for grease lubricated bearings. The supply of lubricant from the cage can be modeled by adding a source term $q_{\text {cage }}(y, t)$ to the continuity relation (5.1):

$$
\frac{\partial \tilde{h}_{\infty}}{\partial t}+\frac{1}{l_{t} \rho_{0}} \frac{\partial \hat{q}_{y}}{\partial y}=\frac{1}{l_{t} \rho_{0}} q_{\text {cage }}(y, t),
$$

where $q_{\text {cage }}(y, t)$ is the rate of mass flow from the cage onto the tracks per unit width in $y$ direction of the track. Unfortunately, the supply rate from the grease reservoirs on the cage is unknown as little is known about grease bleeding. 


\subsubsection{Layer thickness distribution}

In this section the results for the lubricant layer thickness distribution across the track of the spherical roller bearing are discussed. It should be recalled that the lubricant layer thickness is assumed to be distributed evenly along the circumference of the bearing. The results shown in this section represent a cross section of this 3-dimensional distribution. This is illustrated in Figure 6.6.

In Figure 6.7 the layer thickness distribution at different times $t$ is shown for the case of constant force and three different rotational speeds. The values of the parameters used are as given in Table 6.1 for the cases 1, 2 and 3. The initial layer is uniform with thickness $50 \mathrm{~nm}$. After 7.5 minutes the overall layer thickness is decreased by about $3 \mathrm{~nm}$. However, the layer has no longer a uniform thickness. The solution shows a furrow in the center and at both sides of this furrow a levee is formed. The distribution is symmetrical with respect to $y=0$. At the boundaries of the solution domain the layer thickness tends to become very large, which means that liquid accumulates in these areas. The layer thickness in the furrow decreases with time and the levees are pushed to the sides. At the boundaries of the levees a

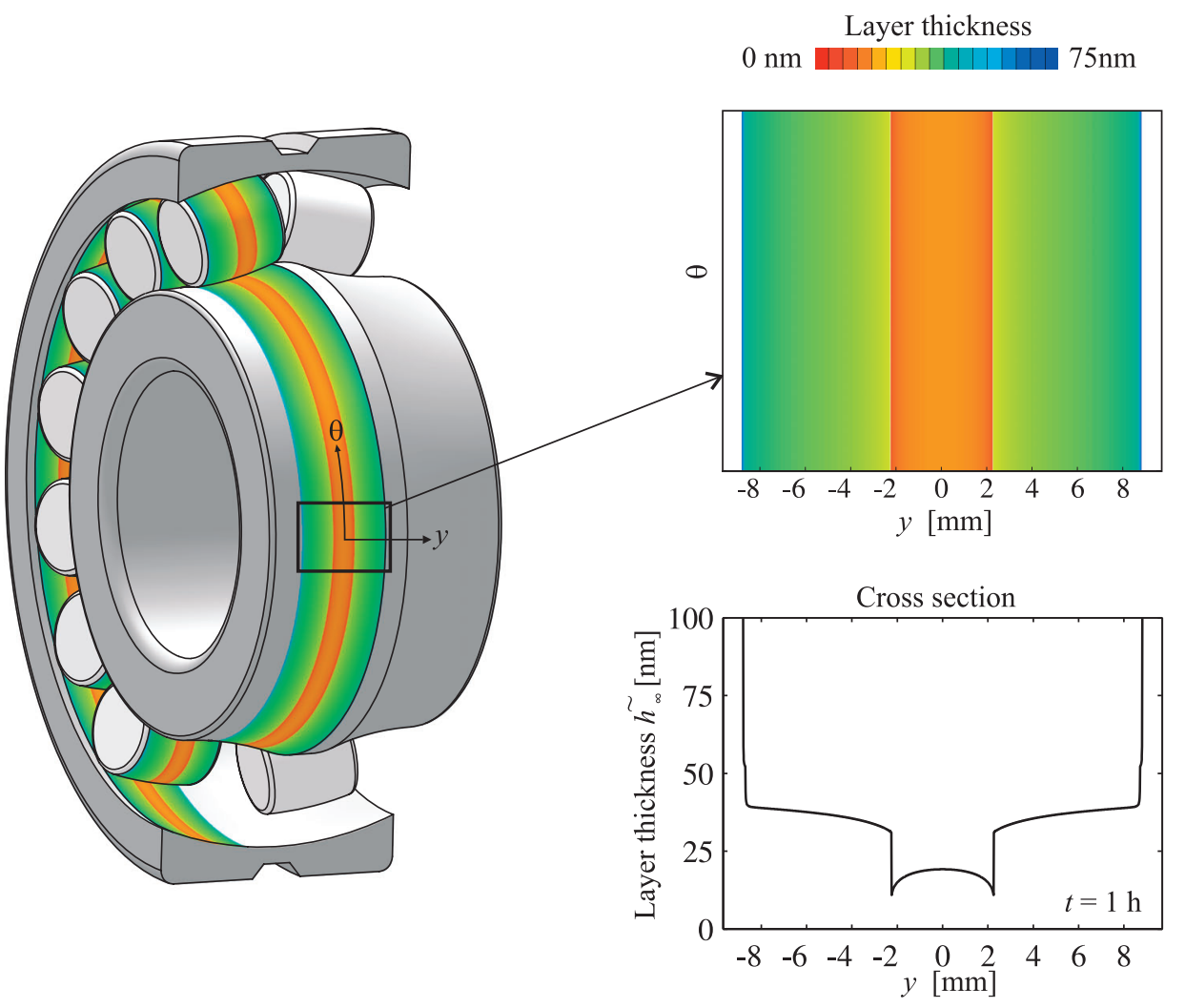

Figure 6.6: Layer thickness distribution at $t=1 \mathrm{~h}$. Results for spherical roller bearing 22317. The operation conditions are given in Table 6.1, Nr. 3. 
discontinuity in the layer thickness occurs. The location of the moving front of a levee is determined by mass conservation, for more details the reader is referred to appendix D.2. Depending on the rotational speed, the front of the levee has moved out of the domain after 1 or 2 hours. The main difference between the solutions for the different rotational velocities is in the width of the furrow and the levees, both of which are larger for larger rotational speeds.

The appearance of the furrow and the levees can be explained by the effect of the relatively large side flow in the lightly loaded zone (zone III in Figure 6.1). The Hertzian contact width is relatively small in this zone. In fact, it is equal to the furrow width. The side flow outside the furrow is smaller, since the layer in this area is only forced in the relatively heavily loaded zones (I and II). Therefore, liquid from the furrow accumulates in this area, resulting in the appearance of the levees. The contact size in zone III is larger for larger centrifugal forces, thus the width of the furrow increases for higher velocities. The model is based on mass conservation. Hence, the levees are larger when the furrow is deeper. As in the lightly loaded zone the pressure and thus the viscosity increase is minimal at the boundaries of the furrow, the minimum layer thickness occurs at these boundaries.

At the border of the solution domain a small ridge is shown. This ridge is the results of the difference of the contact geometry for the inner and the outer raceway. The maximum width of the Hertzian region for the inner raceway contacts is $1.8 \%$ larger than for the outer raceway contacts.

In Figure 6.8 the layer thickness distribution is shown for the parameters of case 5 given in Table 6.1. The parameter values are the same as for the results shown at the bottom of Figure 6.7, except that the bearing load $F_{r}$ is now $2.5 \mathrm{kN}$ rather than $10 \mathrm{kN}$. Notice that the width of the contact in Figure 6.8 is smaller. This is explained by the reduction of the maximum Hertzian contact width due to a smaller bearing load. However, as the effect of the load due to the centrifugal force on the rollers is dominant in the region of the furrow, the results in the central region are not altered due to a change of the bearing load $F_{r}$.

The discontinuities in the predicted layer thickness distribution will not occur in practice. The surface tension tends to reduce the local curvature of the liquid surface. Hence, the actual distribution will be smoother. Secondly, the EHL contacts also contribute to the smoothening of the layers. This smoothening effect has been studied by Damiens et al. [20]. It was shown that it depends on the ratio $s / \lambda$ where $\lambda$ is the wavelength of the layer thickness profile and $s$ the length of the pressurized region at the inlet of the contact up to the Hertzian region. A larger value of the ratio $s / \lambda$ means a larger smoothing effect. For the severely starved cases that are considered here the inlet length $s$ is very small. However, for a discontinuity in the layer thickness the wavelength $\lambda$ is zero. Therefore a local smoothening effect due to the contact pressure is to be expected in these areas. 
Case 1: $F_{r}=10 \mathrm{kN}, \Omega_{i r w}=750 \mathrm{rpm}$

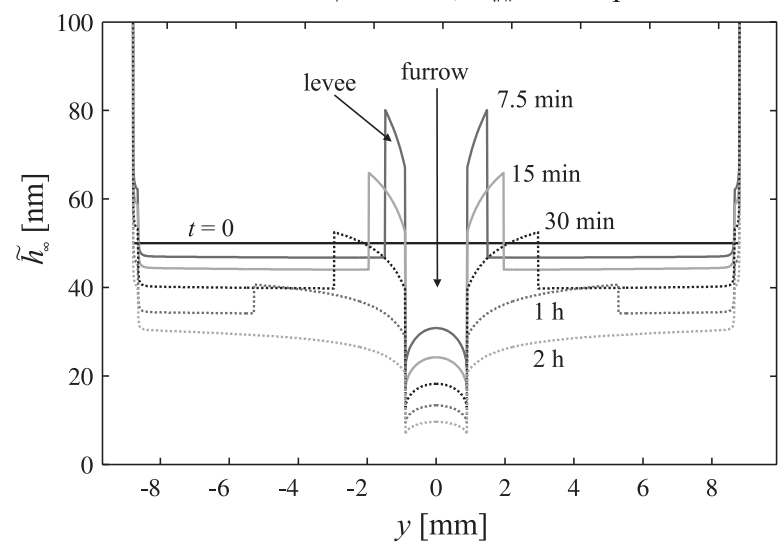

Case 2: $F_{r}=10 \mathrm{kN}, \Omega_{i r w}=1500 \mathrm{rpm}$

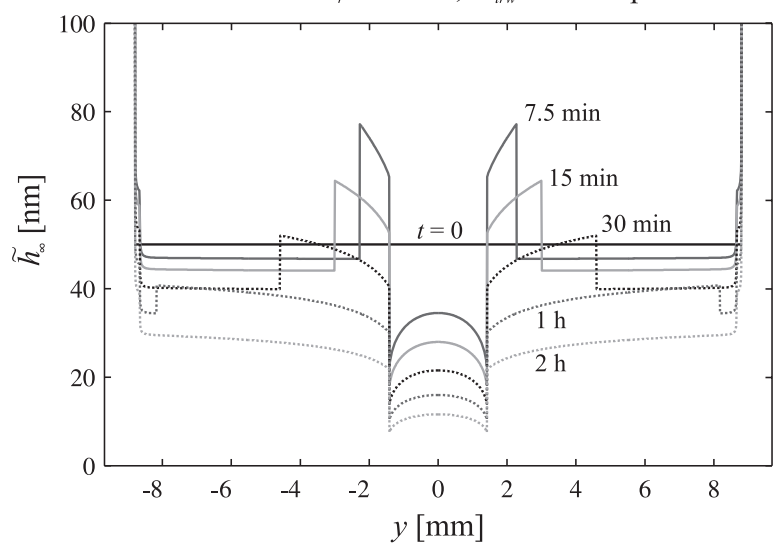

Case 3: $F_{r}=10 \mathrm{kN}, \Omega_{i r w}=3000 \mathrm{rpm}$

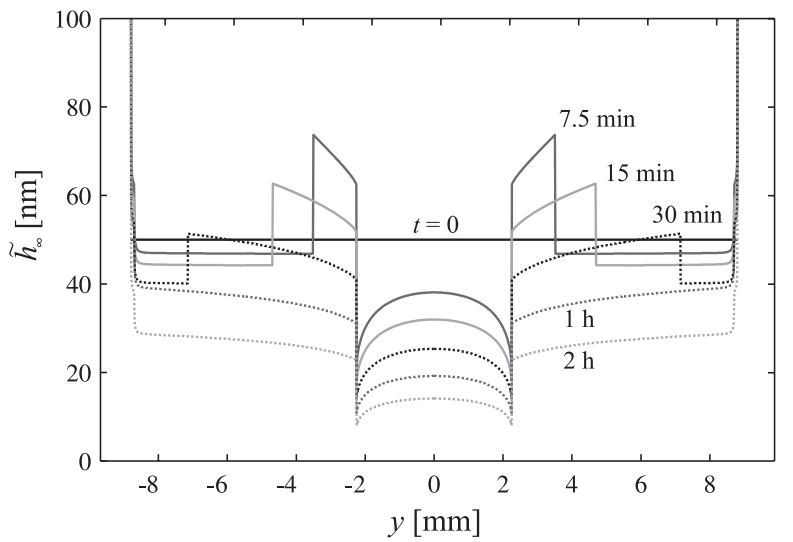

Figure 6.7: Results for spherical roller bearing 22317 illustrating the effect of the velocity on the layer thickness. For the operation conditions, see Table 6.1, $\mathrm{Nr} \mathrm{1,} 2$ and 3, respectively. 


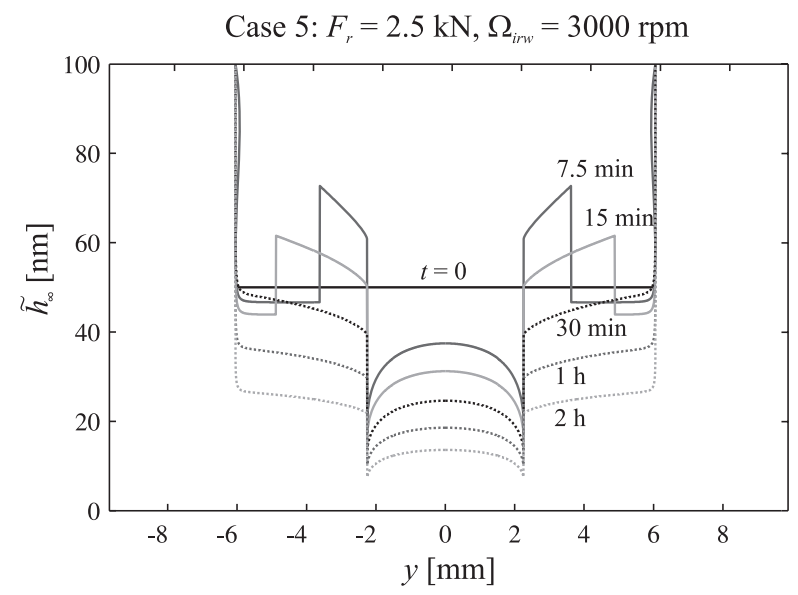

Figure 6.8: Layer thickness distribution for spherical roller bearing 22317 for a load of 2.5 kN. For the operation conditions, see Table 6.1 Nr. 5.

\subsection{Conclusion}

A model has been developed for predicting the change of the supply layer for the long-term operation of an EHL contact under starved conditions. In the present study this model is used to predict the supply layer thickness in a rolling element bearing, taking into account each of the roller-raceway EHL contacts and the variation of the load along the circumference of the bearing.

Results were presented for the layer thickness as a function of time for a deep groove ball bearing and a spherical roller bearing. It is shown that the central layer thickness decay periods predicted for the spherical roller bearing are considerably larger than for the ball bearing. This difference is explained by the different geometry of the contact.

It was also found that the side flow in the lightly loaded zone is relatively large and as a result, the decay time is mainly determined by the magnitude of the centrifugal forces that act on the rollers. This introduces a velocity dependency in the model. On the other hand, the influence of the bearing radial load on the central layer thickness was found to be small. Furthermore, the layer thickness distribution across the track was studied for the spherical roller bearing. In the predicted layer thickness distribution discontinuities were found. Hence, in reality a local smoothening effect due to the contact pressure and surface tension is to be expected in these areas.

The model predicts layer thickness decay periods that are far shorter than the operation periods of bearings commonly found in practice. This can be attributed to the model assuming no replenishment, i.e. it represents a "worst case” scenario. The results show that unless significant replenishment takes place a bearing cannot sustain an adequate lubricant layer. The model can be extended to take into account such effects through a source term in the governing equation. 


\section{Chapter 7}

\section{CONCLUSION AND RECOMMENDATIONS}

\subsection{Conclusion}

When the thickness of the lubricant film in an EHL contact is reduced due to insufficient supply of lubricant, the contact is operating in the so-called starved lubrication regime. This typically occurs in grease lubricated rolling element bearings. As most bearings are lubricated with grease, accurate prediction of the behavior of starved lubricated EHL contact in rolling element bearings is of great importance.

The film thickness in a starved EHL contact is directly related to the thickness of the lubricant layers on the running tracks supplied to the contact. Therefore, accurate models predicting the thickness of these supply layers are a prerequisite to the reliable prediction of the film thickness in rolling element bearings operating in the starved regime. In rolling element bearings the thickness of these supply layers is determined by many effects. In this thesis in particular two effects have been modelled: the centrifugal effect and the contact pressure effect.

The flow of the liquid layers was effectively modelled using the lubrication approximation. For the case of bearing applications it was shown that further simplification is justified, leading to a relative simple equation for the layer thickness, as a function of time and position across the track. The model was successfully validated experimentally using optical interferometry. The model can be used to predict the layer thickness for millions of revolutions and for multiple contacts, without having to solve the starved EHL contact problem for each contact individually. Also, the load variation along the circumference of the bearing and the difference in the geometry of the outer and inner raceway have been taken into account.

It was shown that the flow due to the centrifugal effects is determined by the shape of the surfaces on which the lubricant flows, but is independent of the size of the bearing. For severely starved contacts the layer thickness decay rate is smaller at higher loads. This behavior indicates that optimal design of lubricated contacts, i.e. maximizing the lubricant film, in the case of starved contacts may not necessarily mean minimizing the load. Furthermore, it was found that for severely starved contacts the film thickness decay rate is independent of the velocity. However, for pure radially loaded bearings the decay time is mainly determined by the magnitude of the centrifugal load that acts on the rollers. This introduces a velocity dependency in the model.

The centrifugal effect significantly affects the layer thickness distribution, within the service life of the bearing. However, it was shown that the effect of the contact pressure on 
the layer thickness is large compared the effect of the centrifugal force. The results show that unless significant replenishment takes place a bearing cannot sustain an adequate lubricant layer. With the developed models it is possible to determine the minimum amount of replenishment needed to maintain a sufficiently film thickness for a given type of bearing.

\subsection{Recommendations for future research}

Several recommendations for future research can be made. For further validation of the present model:

- Carry out film thickness measurements for a configuration with multiple EHL contacts. For these experiments a similar setup can be used as for the single contact case. A multiple contact setting can be obtained by pressing several rolling elements mounted in a circle against the glass disk. This is similar to the thrust bearing setup used in one of the classical studies on starved EHL contacts, i.e. the study by Wedeven et al. [70].

- Extension of the current setup so that, the attribution of the spin rate of the ball on the film thickness can be measured. For the case of the ball and the disk the measured starved film thickness is larger than the thickness predicted by the model (Chapter 5). This was attributed to inflow induced by the spin of the ball supplying new lubricant from the sides to the track. When the angle of the ball carriage can be adjusted automatically, the spin rate can be controlled actively. It this way the effect of spin on the film thickness can be studied directly.

- Investigation of the wear marks that occur on the surfaces of the roller and the raceways during bearing operation. These wear marks most likely occur at the locations across the track where the film is minimal and therefore ruptures first. As in this regime the supply of oil to the track is small, the present model can be used to predict the location of the minimal layer thickness, i.e. the location of the wear marks. This could provide some additional validation of the model.

For further development of the model:

- Extension of the model for finite length line contacts. The current film thickness decay model is developed for contacts that are circular or elliptic. However, in for example tapered roller bearings or cylindrical roller bearings, line contact appears. In that case the side flow cannot be calculated using the Hertzian pressure distribution. The calculation of the side flow in starved EHL line contacts requires a separate finite length line contact approach.

- Include the effects of layer smoothening. In Chapter 6 the results for the layer thickness distribution showed discontinuities across the track. It was concluded that, due to surface tension and EHL contact pressure, the real film thickness distribution will be smoother. The effect of surface tension can be included in the 
model using the equations given in Chapter 2. To include the contact pressure effects in the model the real pressure and film thickness profiles in the EHL contact should be determined. To obtain the pressure and film thickness profiles as a function of the position along the circumference of the bearing and as a function of time, the starved EHL problem has to be solved many times, which is computationally expensive. Therefore, a new approach should be developed for the efficient calculation of the pressure and layer thickness distribution for the long term operation.

- Development of a model for the prediction of the regime of boundary or mixed lubrication. Below a certain speed the lubricant is unable to completely separate the steel surfaces, i.e. the film ruptures. Locally in the EHL contact dry zones occur. This is called the boundary or mixed-lubrication regime. In this regime extra heat may be generated and wear of the steel surfaces occur. The surface protection needs to be provided by boundary layers. For the prediction of the occurrence of this regime new models based on physical realistic assumptions are needed, describing the behavior of the lubricant for extremely thin films. Modeling this behavior involves a multi-scale approach.

- Extension of the model with quantitative prediction models for the supply mechanisms. The model presented in Chapter 6 predicts layer thickness decay periods that are far shorter than the operation period of bearings commonly found in practice. This can be attributed to the model assuming no supply of lubricant to the track. The results show that, unless significant lubricant is supplied to the track, a bearing cannot sustain a significant lubricant film. The model can be extended to take into account such effects. 


\section{REFERENCES}

[1] Lubricating Grease Guide, second edition, NLGI, Kansas City, Missouri, 1987.

[2] SKF General Catalogue 6000, Germany, 2005.

[3] Åström, H., Östensen, J. O., and Höglund, E., (1993), “Lubricating Grease Replenishment in an Elastohydrodynamic Point-Contact," ASME Journal of Tribology, vol. 115, no. 3, pp. 501-506.

[4] Barus, C., (1891), "Note on the Dependence of Viscosity on Pressure and Temperature,” Proc. Am. Acad. Arts Sci., vol. 27, no. 13.

[5] Bayada, G., Chambat, M., and El Alaoui, M., (1990), "Variational Formulations and Finite Element Algorithms for Cavitation Problems," ASME Journal of Tribology, vol. 112, no. 2, pp. 398-403.

[6] Booster, E. R. and Wilcock., D. F., (1953), "Minimum Oil Requirements of Ball Bearing,” ASLE Lubrication Engineering, vol. 9, pp. 140-143.

[7] Bos, J., (1995), Frictional Heating of Tribological Contacts, Ph.D. thesis, University of Twente, Enschede, the Netherlands, ISBN 90-9008920-9.

[8] Cameron, A. and Gohar, R., (1966), "Theoretical and Experimental Studies of the Oil Film in Lubricated Point Contact," Proceedings of the Royal Society of London A, vol. 291, no. 1427, pp. 520-536.

[9] Cann, P. M. E., Damiens, B., and Lubrecht, A. A., (2004), “The Transition Between Fully Flooded and Starved Regimes in EHL,” STLE Tribology Transactions, vol. 37, no. 10, pp. 859-864.

[10] Cann, P. M. E. and Lubrecht, A. A., (1999), “An Analysis of the Mechanisms of Grease Lubrication in Rolling Element Bearings,” Lubrication Science, vol. 11, no. 3, pp. 227-245.

[11] Cann, P. M. E. and Lubrecht, A. A., (2003), “The Effect of Transient Loading on Contact Replenishment with Lubricating Greases," in Proceedings of the 30th LeedsLyon Symposium on Tribology, Dalmaz, G. et al. (eds.), Elsevier Tribology Series, vol. 43, pp. 745-750. 
[12] Cann, P. M. E. and Lubrecht, A. A., (2007), "Bearing Performance Limits with Grease Lubrication: the Interaction of Bearing Design, Operating Conditions and Grease Properties,” Journal of Physics D: Applied Physics, vol. 40, no. 18, pp. 54465451.

[13] Cann, P. M. E. and Spikes, H. A., (1992), "Film Thickness Measurements of Lubricating Greases under Normally Starved Conditions,” NLGI spokesman, vol. 56, no. 2, pp. 21-27.

[14] Cann, P. M. E., Spikes, H. A., and Hutchinson, J., (1996), “The Development of a Spacer Layer Imaging Method (SLIM) for Mapping Elastohydrodynamic Contacts,” STLE Tribology Transactions, vol. 39, no. 4, pp. 915-921.

[15] Cann, P. M. E., Webster, M. N., Doner, J. P., Wikstrom, V., and Lugt, P. M., (2007), “Grease Degradation in R0F Bearing Tests," STLE Tribology Transactions, vol. 50, no. 2, pp. 187-197.

[16] Chevalier, F., (1996), Modélisation des Conditions d'alimentation dans les Contactes Élastohydrodynamiques Ponctuels, Phd thesis, I.N.S.A. de Lyon, France.

[17] Chevalier, F., Lubrecht, A. A., Cann, P. M. E., Colin, F., and Dalmaz, G., (1994), "Starved Film Thickness: A Qualitative Explanation," in Proceedings of the 21st Leeds-Lyon Symposium on Tribology, Elseviers Tribology Series, vol. 30, pp. 249257.

[18] Chevalier, F., Lubrecht, A. A., Cann, P. M. E., Colin, F., and Dalmaz, G., (1998), "Film Thickness in Starved EHL Point Contacts," ASME Journal of Tribology, vol. 120, no. 1, pp. 126-133.

[19] Damiens, B., (2003), Modélisation de la Lubrification Sous-Alimentee dans les Contacts Élastohydrodynamiques Elleptiques, Phd thesis, I.N.S.A. de Lyon, France.

[20] Damiens, B., Cann, P. M. E., Venner, C. H., and Lubrecht, A. A., (2003), “Analysis of the Oil Distribution in Starved Elliptical E.H.D. Contacts," in Proceedings of the 29th Leeds-Lyon Symposium on Tribology, Dowson, D. et al. (eds.), Elsevier Tribology Series, vol. 41, pp. 685-691.

[21] Damiens, B., Lubrecht, A. A., and Cann, P. M. E., (2004), "Influence of Cage Clearance on Bearing Lubrication,” STLE Tribology Transactions, vol. 47, no. 1, pp. 2-6.

[22] Damiens, B., Venner, C. H., Cann, P. M. E., and Lubrecht, A. A., (2004), "Starved Lubrication of Elliptical EHD Contacts,” ASME Journal of Tribology, vol. 126, no. 1, pp. 105-111. 
[23] Diez, J. A. and Kondic, L., (2002), “Computing Three-Dimensional Thin Film Flows Including Contact Lines,” Journal of Computational Physics, vol. 183, no. 1, pp. 274 306.

[24] Diez, J. A., Kondic, L., and Bertozzi, A., (2000), “Global Models for Moving Contact Lines,” Physical Review E, vol. 63, no. 1, pp. 011208.

[25] Dowson, D., (1998), History of Tribology, Professional Engineering Publishing Limited, London and Bury St Edmunds, UK, 2nd edn., ISBN 1-86058-070-X.

[26] Dowson, D. and Ehret, P., (1999), "Past, Present and Future Studies in Elastohydrodynamics,” Proceedings of the ImechE; part J., Journal of Engineering Tribology, vol. 213, no. 5, pp. 317-333.

[27] Dowson, D. and Higginson, G. R., (1959), "A Numerical Solution to the Elastohydrodynamic Problem,” J. Mech. Eng. Sci., vol. 1, no. 1, pp. 6-15.

[28] Dowson, D. and Higginson, G. R., (1966), Elastohydrodynamic Lubrication: The Fundamentals of Roller and Gear Lubrication, Pergamon Press, Oxford, Great Britain, ISBN 0080114725.

[29] Elrod, H. G., (1981), “A Cavitation Algorithm,” ASME Journal of Tribology, vol. 103, no. 3, pp. 350-354.

[30] Ertel, A. M., (1939), “Hydrodynamic Lubrication Based on New Principles,” Akad. Nauk SSSR Prikadnaya Mathematica I Mekhanika, vol. 3, no. 2, pp. 41-52.

[31] Foord, C. A., Wedeven, L. D., Westlake, F. J., and Cameron, A., (1969-1970), "Optical Elastohydrodynamics," Proceedings of the ImechE; part J., Journal of Engineering Tribology, vol. 184, no. 1, pp. 487-505.

[32] Gershuni, L., Larson, M. G., and Lugt, P. M., (2008), "Lubricant Replenishment in Rolling Bearing Contacts,” STLE Tribology Transactions, vol. 51, no. 5, pp. 643-651.

[33] Gohar, R. and Cameron, A., (1963), “Optical Measurement of Oil Film Thickness under Elasto-Hydrodynamic Lubrication,” Nature, vol. 200, pp. 458-459.

[34] Grubin, A. N. and Inogradova, I. E., (1949), Kh, F. Ketova (Ed.), Central Scientific Research Institute for Technology and Mechanical Engineering (Moscow), Book No. 30, (DSIR translation No. 337).

[35] Hamrock, B. J. and Dowson, D., (1975), "Isothermal Elastohydrodynamic Lubrication of Point Contacts - Part I - Theory,” Tech. rep., NASA, USA.

[36] Harris, T. A., (1984), Rolling Bearing Analysis, John Wiley \& Sons, 2nd edn., ISBN 0-471-79979-3. 
[37] Hertz, H., (1881), “Über die Berührung Fester Elastischer Körper,” Journal für die reine und angewandte Mathematik, vol. 92, pp. 156-171.

[38] Howell, P. D., (2003), "Surface-Tension-Driven Flow on a Moving Curved Surface,” Journal of Engineering Mathematics, vol. 45, no. 3, pp. 283-308.

[39] Hurley, S. and Cann, P. M. E., (2003), "Infrared Spectroscopic Analysis of a GreaseLubricated Rolling Contact,” NLGI spokesman, vol. 67, no. 6, pp. 26-32.

[40] Jacod, B. C., Pubilier, F., Cann, P. M. E., and Lubrecht, A. A., (1999), “An Analysis of Track Replenishment Mechanisms in the Starved Regime," in Proceedings of the 25th Leeds-Lyon Symposium on Tribology, Dowson, D. et al. (eds.), Elsevier Tribology Series, vol. 36, pp. 483-492.

[41] Johnson, K. L., (1985), Contact Mechanics, Cambridge University Press, UK, ISBN 0 521347963.

[42] Johnston, G. J., Wayte, R., and Spikes, H. A., (1991), "The Measurement and Study of Very Thin Lubricant Films in Concentrated Contacts," STLE Tribology Transactions, vol. 34, no. 2, pp. 187-194.

[43] Kendall, D. L. R., Williamson, B. P., and Cann, P. M. E., (1993), “The Influence of Grease Composition on Film Thickness in EHD Contacts,” NLGI Spokesman, vol. 57, no. 8, pp. 13-18.

[44] Kingsbury, E., (1973), “Cross Flow in a Starved EHD Contact,” ASLE Transactions, vol. 16, no. 4, pp. 276-280.

[45] Kingsbury, E., (1985), "Parched Elastohydrodynamic Lubrication,” ASME Journal of Tribology, vol. 107, pp. 229-233.

[46] Kuznetsov, A. A. and Martynov, V. M., (1972), “Contact Angle of Lubricating Oils at Elevated Temperatures,” Chemistry and Technology of Fuels and Oils, vol. 8, no. 10, pp. 773-776.

[47] Lee-Prudhoe, I., Venner, C. H., Cann, P. M. E., and Spikes, H., (2006), "Experimental and Theoretical Approaches to Thin Film Lubrication Problems," in IUTAM Symposium on Elastohydrodynamics and Micro-elastohydrodynamics, Snidle, R. W. and Evans, H. P. (eds.), vol. 134, pp. 241-255.

[48] Lubrecht, A. A., (1987), The Numerical Solution of Elastohydrodynamically Lubricated Line- and Point Contact Problem Using Multigrid Techniques, Ph.D. thesis, University of Twente, Enschede, the Netherlands, ISBN 90-9001583-3.

[49] Lugt, P. M., (2009), “A Review on Grease Lubrication in Rolling Bearings,” STLE Tribology Transactions, vol. 52, no. 4, pp. $470-480$. 
[50] Lugt, P. M., Velickov, S., and Tripp, J. H., (2009), "On the Chaotic Behavior of Grease Lubrication in Rolling Bearings,” Tribology Transactions, vol. 52, no. 5, pp. 581-590.

[51] Moes, H., (2000), “Lubrication and Beyond,” Lecture notes , code 115531, University of Twente, Enschede, the Netherlands.

[52] Morales-Espejel, G. E. and Wemekamp, A. W., (2008), "Ertel-Grubin Methods in Elastohydrodynamic Lubrication - A Review," Proceedings of the ImechE; part J., Journal of Engineering Tribology, vol. 222, no. 1, pp. 15-34.

[53] Myers, T. G. and Charpin, J. P. F., (2001), "The Effect of the Coriolis Force on Axisymmetric Rotating Thin Film Flows,” International Journal of Non-Linear Mechanics, vol. 36, no. 4, pp. 629-635.

[54] Myers, T. G., Charpin, J. P. F., and Chapman, S. J., (2002), "The Flow and Solidification of a Thin Fluid Film on an Arbitrary Three-Dimensional Surface," Physics of Fluids, vol. 14, no. 8, pp. 2788-2803.

[55] O’Brien, S. B. G. and Schwartz, L. W., (2002), in Encyclopedia of Surface and Colloid Science, Hubbard, A. (ed.), chap. Theory and Modeling of Thin Film Flows, pp. 5283-5297, M. Dekker, New york, ISBN 0824706331.

[56] Petrusevich, A. I., (1951), "Fundamental Conclusions from the Contact Hydrodynamic Theory of Lubrication,” Izv. Akad. Hauk SSR (ONT), vol. 2, pp. 209223.

[57] Rajagopal, K. R. and Szeri, A. Z., (2003), “On an Inconsistency in the Derivation of the Equations of Elastohydrodynamic Lubrication," Proceedings: Mathematical, Physical and Engineering Sciences, vol. 459, no. 2039, pp. 2771-2786.

[58] Reynolds, O., (1886), “On the Theory of Lubrication and Its Application to $\mathrm{Mr}$ Beauchamp Tower's Experiments, Including an Experimental Determination of the Viscosity of Olive Oil,” Phil. Trans. R. Soc., London, UK, vol. 177, pp. 157-234.

[59] Roelands, C. J. A., (1966), Correlational Aspects of the Viscosity-TemperaturePressure Relationship of Lubrication Oils, Ph.D. thesis, Technische Hogeschool Delft, the Netherlands.

[60] Schäfer, C. T., Giese, P., Rowe, W. B., and Woolley, N. H., (2000), "Elastohydrodynamically Lubricated Line Contact Based on the Navier-Stokes Equations.," in Thinning Films and Tribological Interfaces. Proc. 26th Leeds-Lyon Symposium, Dowson, D. et al. (eds.), Tribology series, vol. 28, pp. 57-68, Elsevier.

[61] Schwartz, L. W. and Weidner, D. E., (1995), "Modeling of Coating Flows on Curved Surfaces,” Journal of Engineering Mathematics, vol. 29, no. 1, pp. 91-103. 
[62] Seeton, C., (2006), "Viscosity-Temperature Correlation for Liquids," Tribology Letters, vol. 22, no. 1, pp. 67-78.

[63] Snoeijer, J. H., (2006), "Free-surface flows with large slopes: Beyond lubrication theory,” Phys. of Fluids, vol. 18, no. 2, pp. 021701-4.

[64] Valery Roy, R., Roberts, A. J., and Simpson, M. E., (2002), “A Lubrication Model of Coating Flows Over a Curved Substrate in Space,” Journal of Fluid Mechanics, vol. 454, pp. 235-261.

[65] Venner, C. H., (1991), Multilevel Solution of the EHL Line and Point Contact Problem, Ph.D. thesis, University of Twente, ISBN 90-9003974-0.

[66] Venner, C. H. and Lubrecht, A. A., (1994), "Numerical Simulation of a Transverse Ridge in a Circular EHL Contact under Rolling/Sliding,” ASME Journal of Tribology, vol. 116, no. 4, pp. 751-761.

[67] Venner, C. H. and Lubrecht, A. A., (2000), Multilevel Methods in Lubrication, Tribology Series, vol. 37, Elsevier, Amsterdam, ISBN 0-444-50503-2.

[68] Venner, C. H., Popovici, G., Lugt, P. M., and Organisciak, M., (2008), "Film Thickness Modulations in Starved Elastohydrodynamically Lubricated Contacts Induced by Time-Varying Lubricant Supply,” ASME Journal of Tribology, vol. 130, no. 4, pp. 041501.

[69] Wedeven, L. D., (1970), Optical Measurements in Elastohydrodynamic Rolling Contact Bearings, Ph.D. thesis, Imperial College, London, England.

[70] Wedeven, L. D., Evans, D., and Cameron, A., (1971), “Optical Analysis of Ball Bearing Starvation,” Journal of Lubrication Technology, vol. 93, no. 3, pp. 349-363.

[71] Wijnant, Y.H., (1998), Contact Dynamics in the Field of Elastohydrodynamic Lubrication, Ph.D. thesis, University of Twente, Enschede, the Netherlands, ISBN 9036512239.

[72] Wikström, V. and Höglund, E., (1996), "Starting and Steady-State Friction Torque of Grease-Lubricated Rolling Element Bearings at Low Temperatures. 1. A Parameter Study,” STLE Tribology Transactions, vol. 39, no. 3, pp. 517-526.

[73] Wikström, V. and Höglund, E., (1996), “Starting and Steady-State Friction Torque of Grease-Lubricated Rolling Element Bearings at Low Temperatures. 2. Correlation with Less-Complex Test Methods,” STLE Tribology Transactions, vol. 39, no. 3, pp. 684-690.

[74] Wikström, V. and Jacobson, B., (1997), "Loss of Lubricant from Oil-Lubricated Near-Starved Spherical Roller Bearings,” Proceedings of the ImechE; part J., Journal of Engineering Tribology, vol. 211, no. J1, pp. 51-66. 
[75] Wilson, A. R., (1979), “The Relative Film Thickness of Grease and Oil Films in Rolling Bearings," Proceedings of the ImechE; part J., Journal of Engineering Tribology, vol. 193, pp. 185-192.

[76] Wu, M., Ho, S., and Forbus, T., (2006), in Practical Advances in Petroleum Processing, Hsu, C. S. and Robinson, P. R. (eds.), chap. 17, Synthetic Lubricant Base Stock Processes and Products, pp. 553-577, Springer, New York, ISBN 978-0-38725811-9.

[77] van Zoelen, M. T., Venner, C. H., and Lugt, P. M., (2008), "Free Surface Thin Layer Flow on Bearing Raceways," ASME Journal of Tribology, vol. 130, no. 2, pp. 021802.

[78] van Zoelen, M. T., Venner, C. H., and Lugt, P. M., (2009), "Free Surface Thin Layer Flow in Bearings Induced by Centrifugal Effects," STLE Tribology Transactions, in press.

[79] van Zoelen, M. T., Venner, C. H., and Lugt, P. M., (2009), "Prediction of Film Thickness Decay in Starved EHL Contacts Using a Thin Layer Flow Model," Proceedings of the ImechE; part J., Journal of Engineering Tribology, vol. 223, pp. 541-552. 


\section{Appendix A}

\section{A.1 Method of characteristics}

In this section the quasi-linear PDE (2.37) is solved using the method of characteristics. For convenience the bars are omitted and the equation is written in the following form:

$$
\frac{1}{r} \frac{\partial}{\partial s}\left(\frac{1}{3} h^{3} q\right)+\frac{\partial h}{\partial t}=0 \Rightarrow-\frac{1}{3} \frac{1}{r} \frac{d q}{d s} h^{3}=\frac{\partial h}{\partial t}+\frac{\partial h}{\partial s} \frac{q}{r} h^{2}
$$

with:

$$
r=r(s) \text {, and } h=h(s, t) .
$$

The solution surface is defined by the vector:

$$
\left(\begin{array}{l}
t(\sigma, \xi) \\
s(\sigma, \xi) \\
h(\sigma, \xi)
\end{array}\right),
$$

where the characteristic curves, parameterized by $\xi$, emerge from the initial curve which is parameterized by $\sigma$, see Figure 2.3. Using the chain rule the derivative of $h$ with respect to $\xi$ is written as:

$$
\frac{d h}{d \xi}=\frac{\partial h}{\partial t} \frac{d t}{d \xi}+\frac{\partial h}{\partial s} \frac{d s}{d \xi} .
$$

Equation (A.1) is similar to Eq. (A.4) and after comparison the following coupled system of differential equations is obtained:

$$
\begin{aligned}
& \frac{d t}{d \xi}=1, \\
& \frac{d s}{d \xi}=\frac{q}{r} h^{2}, \\
& \frac{d h}{d \xi}=-\frac{1}{3} \frac{1}{r} \frac{d q}{d s} h^{3} .
\end{aligned}
$$

The following boundary conditions are defined:

$$
\xi=0 \quad \rightarrow \quad t=t_{0}(\sigma), \quad s=s_{0}(\sigma), \quad h=h_{0}(\sigma)
$$


Thus, $t_{0}, s_{0}$ and $h_{0}$ are the starting positions of the characteristic curves on $t, s$ and $h$. Integration of the first equation of Eq. (A.5) and application of the initial condition gives:

$$
t(\sigma, \xi)=\xi+t_{0}
$$

The combination of the last two equations of Eq. (A.5) leads to:

$$
\frac{d h}{d s}=-\frac{1}{3} \frac{h}{q} \frac{d q}{d s}
$$

Separation of variables and integration over the characteristic curves gives:

$$
\int \frac{1}{h} d h=-\frac{1}{3} \int\left(\frac{1}{q} \frac{d q}{d s}\right) d s \quad \Rightarrow \ln |h|=-\frac{1}{3} \ln |q|+f_{1}(\sigma),
$$

where $f_{1}(\sigma)$ is an arbitrary function. Application of the boundary conditions gives:

$$
h(\sigma, \xi)=\left(\frac{q\left(s_{0}\right)}{q(s)}\right)^{\frac{1}{3}} h_{0}
$$

The next step is to find a solution for $s(\sigma, \xi)$. Substitution of Eq. (A.10) into the second equation of Eq. (A.5) gives:

$$
\frac{r(s)}{(q(s))^{\frac{1}{3}}} \frac{d s}{d \xi}=\left(q\left(s_{0}\right)\right)^{\frac{2}{3}} h_{0}^{2} .
$$

Integration over the characteristic curves gives:

$$
\int \frac{r(s)}{(q(s))^{\frac{1}{3}}} d s=\left(q\left(s_{0}\right)\right)^{\frac{2}{3}} \xi h_{0}^{2}+f_{2}(\sigma)
$$

where $f_{2}(\sigma)$ is an arbitrary function. Substitution of the boundary conditions (Eq. (A.6)) and Eq. (A.7) gives the equation for the characteristic projections:

$$
\int_{s_{0}}^{s} \frac{r(\phi)}{q(\phi)^{\frac{1}{3}}} d \phi=\left(q\left(s_{0}\right)\right)^{\frac{2}{3}}\left(t-t_{0}\right) h_{0}^{2}
$$

where $\phi$ is an integration variable. 


\section{A.2 Discrete equations}

In this section the discretization of Eq. (2.33) and Eq. (2.35) is discussed for the case of a axisymmetric layer, i.e. $Q_{\theta}=0$. For convenience the bars on the dimensionless variables are omitted. The discretization method used to solve Eq. (2.33) is second-order accurate in space. The diffusivity term is discretized using the positivity preserving scheme (PPS) on a uniform grid proposed by Diez et al. [23]. The time discretization is performed using an explicit first order Euler scheme. Using this scheme, the time step $\Delta t$ has to be much smaller than the space step $\Delta t<\mathrm{O}(\Delta s)^{4}$ in order to maintain numerical stability. Hence, for fine grids it becomes necessary to use partially-implicit methods. However, within the scope of this work it is sufficient to use the Euler scheme:

$$
h_{i}^{k+1}=h_{i}^{k}-\frac{1}{r_{i}} \frac{\Delta t}{\Delta s}\left(r_{i+1 / 2} Q_{i+1 / 2}^{(s)}-r_{i-1 / 2} Q_{i-1 / 2}^{(s)}\right),
$$

where $i$ numbers the nodes in $s$ direction, respectively and $k$ is the time step number. The flux component $Q_{s}$, see Eq. (2.35), is reorganized and discretized according to:

$$
\begin{aligned}
& Q_{i+1 / 2}^{(s)}=\frac{1}{3} H_{i+1 / 2} \frac{h_{i}+h_{i+1}}{2}\left[f_{s}+C \kappa^{\prime}\right]_{i+1 / 2}+\frac{1}{3} G_{i+1 / 2} \varepsilon\left[f_{n}\right]_{i+1 / 2} \frac{h_{i+1}-h_{i}}{\Delta s} \\
& +\frac{1}{3} D_{i+1 / 2} \varepsilon C \frac{\left.\Delta^{2} h\right|_{i+1}-\left.\Delta^{2} h\right|_{i}}{\Delta s} .
\end{aligned}
$$

The discrete surface Laplacian is defined according to:

$$
\left.\Delta^{2} h\right|_{i}=\frac{1}{r_{i}} \frac{1}{(\Delta s)^{2}}\left(r_{i+1 / 2}\left(h_{i+1}-h_{i}\right)-r_{i-1 / 2}\left(h_{i}-h_{i-1}\right)\right) .
$$

The nonlinear terms read:

$$
D_{i+1 / 2}=2 \frac{h_{i}^{2} h_{i+1}^{2}}{h_{i}+h_{i+1}}, \quad G_{i+1 / 2}=\frac{\left(h_{i}\right)^{3}+\left(h_{i+1}\right)^{3}}{2}, \quad H_{i+1 / 2}=\frac{\left(h_{i}\right)^{2}+\left(h_{i+1}\right)^{2}}{2} .
$$




\section{A.3 Numerical accuracy}

For the numerically calculated layer thickness distribution presented in section 2.5, in Figure A.1 the initial layer thickness and the solution at $t=1 / 128 \mathrm{~h}$ is shown, calculated on various grid size $\Delta \bar{s}=1 / 64,1 / 128,1 / 256,1 / 512$ and scaled time step size $\Delta \bar{t}=1.4 \cdot 10^{-3}, 1.4 \cdot 10^{-4}, 1.4 \cdot 10^{-5}, 1.4 \cdot 10^{-6}$, respectively. Except for the bump region, the layer thickness calculated on the different grids is indistinguishable. At the bump the maximum value decreases with decreasing grid and time step size. The solutions for the three finest grid levels clearly show second order behavior. The same behavior was found for other times. The difference between the solutions for the two finest grids is small. Therefore, a grid size of $\Delta \bar{s}=1 / 256$ and a time step size of $\Delta \bar{t}=1.4 \cdot 10^{-5}$ is sufficient for this example.

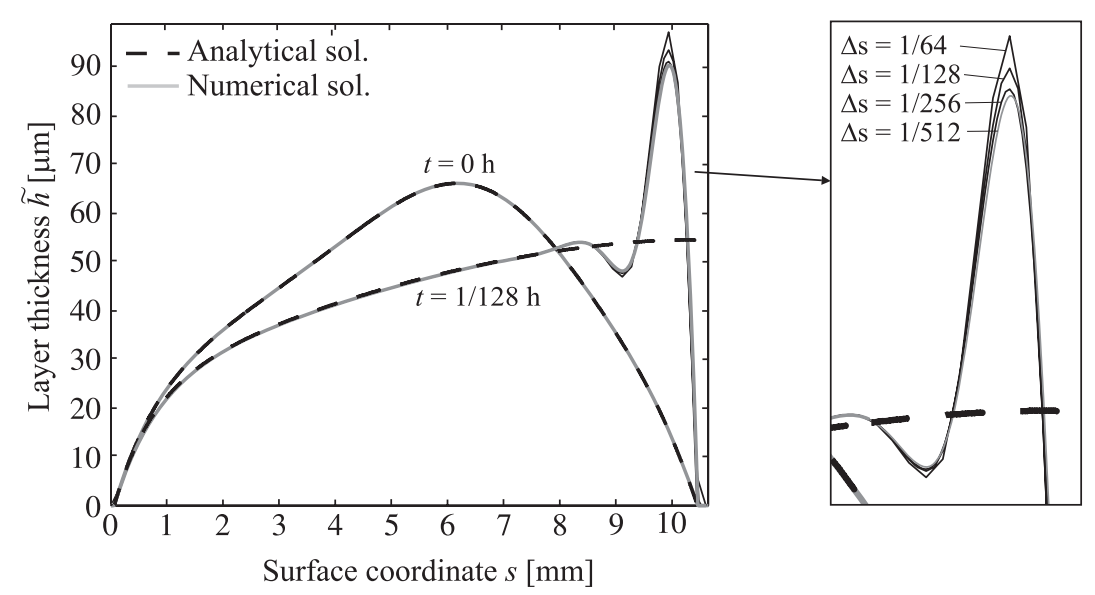

Figure A.1: Solution of the layer thickness calculated on various grid and time step sizes. 


\section{Appendix B}

In this appendix the equations are derived for the inertia forces, acting on the fluid layer on the surface of a rolling element, induced by the motion of this rolling element in a bearing. In the layer thickness model, presented in Chapter 3, the body force components are defined in a coordinate system that is fixed to the roller. In the next section, the transformation between this local coordinate system of the roller and the inertia frame will be defined.

\section{B.1 Coordinate transformations}

In Figure B.1 four Cartesian coordinate systems are shown. The coordinate systems $\mathbf{x}_{1}=\left(x_{1}, y_{1}, z_{1}\right)$ and $\mathbf{x}_{\text {rol }}$ represent the inertia frame and the local coordinate system of the roller, respectively. See also Figure 3.1. The coordinate system $\mathbf{x}_{2}$ is fixed to the cage of the bearing and is rotating at a speed $\Omega_{c a}$, relative to the inertia frame. The centre of the coordinate system $\mathbf{x}_{3}$ is positioned at the centre of the roller at a distance $R_{\text {crol }}$ relative to axis $z_{2}$. The axis $z_{3}$ coincides with the axis of rotation of the roller. $\gamma$ denotes the angle between the axis of rotation of the roller $z_{\text {rol }}$ and the cage $z_{2}$, respectively. The coordinate system $\mathbf{x}_{\text {rol }}$ is rotating around the axis $z_{\text {rol }}$, at a negative speed $-\Omega_{\text {rol }}$ relative to $\mathbf{x}_{3}$.

The coordinate transformations are defined according to:

$$
\begin{array}{lll}
\mathbf{x}_{1}=\mathbf{R}_{1} \mathbf{x}_{2}, \quad \mathbf{x}_{2}=\mathbf{R}_{2} \mathbf{x}_{3}+\mathbf{T}_{1}, & \mathbf{x}_{3}=\mathbf{R}_{3} \mathbf{x}_{\text {rol }}, \\
\Rightarrow \quad \mathbf{x}_{1}=\mathbf{R}_{4} \mathbf{x}_{\text {rol }}+\mathbf{R}_{1} \mathbf{T}_{1}, \quad \text { with } \quad \mathbf{R}_{4}= & \mathbf{R}_{1} \mathbf{R}_{2} \mathbf{R}_{3},
\end{array}
$$

where the rotation matrices and the translation vector read:

$$
\begin{array}{rlrl}
\mathbf{R}_{1} & =\left[\begin{array}{lll}
\cos \left(\Omega_{c a} t\right) & -\sin \left(\Omega_{c a} t\right) & 0 \\
\sin \left(\Omega_{c a} t\right) & \cos \left(\Omega_{c a} t\right) & 0 \\
0 & 0 & 1
\end{array}\right], & \mathbf{R}_{2}=\left[\begin{array}{ccc}
\cos (\gamma) & 0 & \sin (\gamma) \\
0 & 1 & 0 \\
-\sin (\gamma) & 0 & \cos (\gamma)
\end{array}\right], \\
\mathbf{R}_{3}=\left[\begin{array}{ccc}
\cos \left(-\Omega_{\text {rol }} t\right) & -\sin \left(-\Omega_{\text {rol }} t\right) & 0 \\
\sin \left(-\Omega_{\text {rol }} t\right) & \cos \left(-\Omega_{\text {rol }} t\right) & 0 \\
0 & 0 & 1
\end{array}\right], & \mathbf{T}_{1}=\left[\begin{array}{c}
R_{c r o l} \\
0 \\
0
\end{array}\right] .
\end{array}
$$




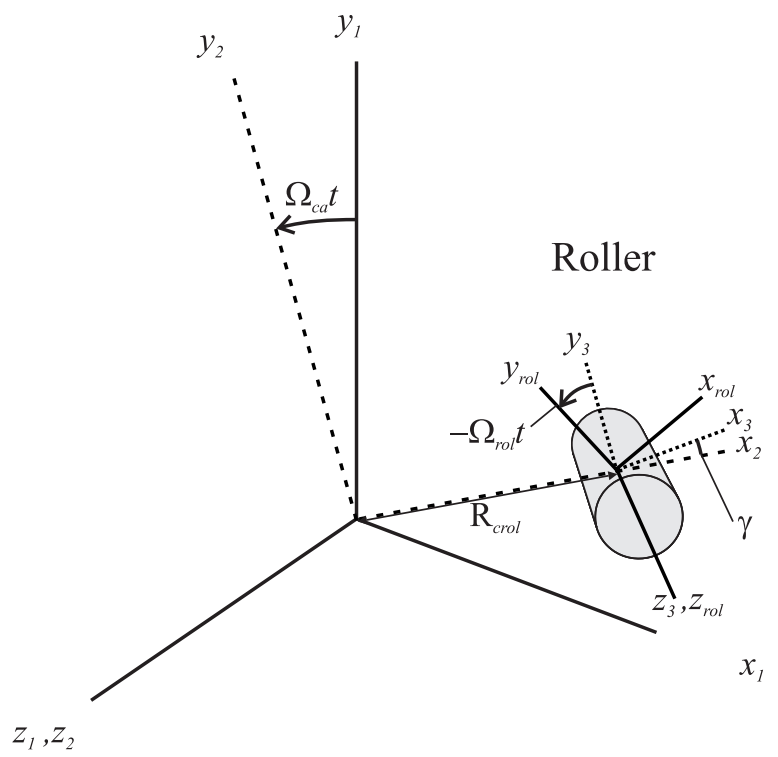

Figure B.1: Transformations between the inertia frame and the local coordinate system of a roller.

\section{B.2 Acceleration vector of a fluid particle}

Consider a fluid particle which location is defined by the vector $\mathbf{r}_{\mathbf{1}}$ and $\mathbf{r}_{\text {rol }}$ in the $\mathbf{x}_{\mathbf{1}}$ and the $\mathbf{x}_{\text {rol }}$ coordinate system, respectively. According to Eq. (B.1) the relation between $\mathbf{r}_{\mathbf{1}}$ and $\mathbf{r}_{\text {rol }}$ reads:

$$
\mathbf{r}_{1}=\mathbf{R}_{4} \mathbf{r}_{\text {rol }}+\mathbf{R}_{1} \mathbf{T}_{1}
$$

The velocity and acceleration of the fluid particle relative to the inertia frame are found by differentiation of Eq. (B.3) with respect to time $t$ :

$$
\begin{aligned}
& \dot{\mathbf{r}}_{1}=\mathbf{R}_{4} \dot{\mathbf{r}}_{\text {rol }}+\dot{\mathbf{R}}_{4} \mathbf{r}_{\text {rol }}+\dot{\mathbf{R}}_{1} \mathbf{T}_{1}, \\
& \ddot{\mathbf{r}}_{1}=\mathbf{R}_{4} \ddot{\mathbf{r}}_{\text {rol }}+2 \dot{\mathbf{R}}_{4} \dot{\mathbf{r}}_{\text {rol }}+\ddot{\mathbf{R}}_{4} \mathbf{r}_{\text {rol }}+\ddot{\mathbf{R}}_{1} \mathbf{T}_{1} .
\end{aligned}
$$

For the application considered, it is reasonable to assume that the acceleration and velocity of the fluid particle, relative to the underlying solid surface, are negligible compared to the acceleration and velocity of the solid surface relative to the inertia frame. Hence, the acceleration vector of the fluid particle reduces to:

$$
\begin{aligned}
\ddot{\mathbf{r}}_{1} & =\mathbf{R}_{4} \ddot{\mathbf{r}}_{\text {rol }}+2 \dot{\mathbf{R}}_{4} \dot{\mathbf{r}}_{\text {rol }}+\ddot{\mathbf{R}}_{4} \mathbf{r}_{\text {rol }}+\ddot{\mathbf{R}}_{1} \mathbf{T}_{1} \\
& \approx \ddot{\mathbf{R}}_{4} \mathbf{r}_{\text {rol }}+\ddot{\mathbf{R}}_{1} \mathbf{T}_{1} .
\end{aligned}
$$




\section{B.3 Local force vector}

The body force due to inertia, which acts on the fluid with a density $\rho$, is defined by:

$$
\mathbf{f}_{1}=-\rho \ddot{\mathbf{r}_{1}} .
$$

The body force vector is transformed to the reference frame of the roller $\mathbf{x}_{\text {rol }}$ :

$$
\mathbf{f}_{\text {rol }}=\mathbf{R}_{3}{ }^{-1} \mathbf{R}_{2}{ }^{-1} \mathbf{R}_{1}{ }^{-1} \mathbf{f}_{1} .
$$

Substitution of Eq. (B.6) and Eq. (B.5) into Eq. (B.7) gives:

$$
\mathbf{f}_{\text {rol }}=-\rho \mathbf{R}_{3}^{-1} \mathbf{R}_{2}^{-1} \mathbf{R}_{1}^{-1} \ddot{\mathbf{R}}_{4} \mathbf{r}_{\text {rol }}-\rho \mathbf{R}_{3}^{-1} \mathbf{R}_{2}^{-1} \mathbf{R}_{1}^{-1} \ddot{\mathbf{R}}_{1} \mathbf{T}_{1} .
$$

The force vector components defined in the local surface coordinate system of the roller $(s, \theta)$, see Figure 3.1, read:

$$
f_{s, r o l}=\mathbf{f}_{\text {rol }} \cdot \mathbf{e}_{1}, \quad f_{\theta, \text { rol }}=\mathbf{f}_{\text {rol }} \cdot \mathbf{e}_{2}, \quad f_{n, \text { rol }}=\mathbf{f}_{\text {rol }} \cdot \mathbf{n},
$$

where:

$$
\begin{array}{ll}
\mathbf{r}_{\text {rol }}=\left[\begin{array}{c}
r(s) \cos (\theta) \\
r(s) \sin (\theta) \\
z(s)
\end{array}\right], & \mathbf{e}_{1}=\frac{\partial \mathbf{r}_{\text {rol }}}{\partial s} /\left|\frac{\partial \mathbf{r}_{\text {rol }}}{\partial s}\right|=\left[\begin{array}{c}
r^{\prime}(s) \cos (\theta) \\
r^{\prime}(s) \sin (\theta) \\
z^{\prime}(s)
\end{array}\right], \\
\mathbf{e}_{2}=\frac{\partial \mathbf{r}_{\text {rol }}}{\partial \theta} /\left|\frac{\partial \mathbf{r}_{\text {rol }}}{\partial \theta}\right|=\left[\begin{array}{c}
-\sin (\theta) \\
\cos (\theta) \\
0
\end{array}\right], \quad \mathbf{n}=-\mathbf{e}_{1} \times \mathbf{e}_{2}=\left[\begin{array}{c}
z^{\prime}(s) \cos (\theta) \\
z^{\prime}(s) \sin (\theta) \\
-r^{\prime}(s)
\end{array}\right] .
\end{array}
$$

Substitution of Eq. (B.10), Eq. (B.8) and Eq. (B.2) into Eq. (B.9) gives the equations for the body force terms in the local coordinate system on the surface of the roller. It is found that these force components are very involved. They depend on the position $\theta$ and the time $t$. However, when they are averaged over the circumference, using Eq. (3.12), the force terms are dramatically simplified and are only a function of $s$ :

$$
\begin{aligned}
& \hat{f}_{s, r o l}(s)=\rho \Omega_{c a}{ }^{2}\left(\sin ^{2}(\gamma) z(s)+\sin (\gamma) R_{c r o l}\right) z^{\prime}(s)+\ldots \\
& \left(\left(\frac{1}{2} \cos ^{2}(\gamma)+\frac{1}{2}\right) \Omega_{c a}{ }^{2}-2 \Omega_{c a} \Omega_{r o l} \cos (\gamma)+\Omega_{r o l}{ }^{2}\right) \rho r^{\prime}(s) r(s), \\
& \hat{f}_{\theta, r o l}(s)=0, \\
& \hat{f}_{n, r o l}(s)=\rho \Omega_{c a}{ }^{2}\left(-\sin ^{2}(\gamma) z(s)-\sin (\gamma) R_{c r o l}\right) r^{\prime}(s)+\ldots \\
& \left(\left(\frac{1}{2} \cos ^{2}(\gamma)+\frac{1}{2}\right) \Omega_{c a}{ }^{2}-2 \Omega_{c a} \Omega_{r o l} \cos (\gamma)+\Omega_{r o l}{ }^{2}\right) \rho z^{\prime}(s) r(s) .
\end{aligned}
$$




\section{Appendix C}

\section{C.1 Derivation of the asymptotic flux}

In this section $\hat{Q}_{Y}$ is derived for heavily starved contacts. Substitution of the film thickness (Eq. (5.15)) and the pressure (Eq. (5.17)) into Eq. (5.10) gives:

$$
\hat{Q}_{Y, k}(Y)=\int_{\bar{a}^{-}}^{\bar{a}^{+}}\left(\bar{\eta}^{-1} \bar{\rho}^{-2} \frac{Y}{P_{H}}\right) d X .
$$

Coordinate $\varphi$ is introduced and the following coordinate transformation is defined:

$$
X=\bar{a}^{+} \sin (\varphi)=\sqrt{1-Y^{2}} \sin (\varphi) .
$$

Differentiation of Eq. (C.2) with respect to $\varphi$ gives:

$$
d X=\sqrt{1-Y^{2}} \cos (\varphi) d \varphi .
$$

Substitution of Eq. (C.2) into Eq. (5.17) gives:

$$
P_{H}(\varphi, Y)=\sqrt{1-Y^{2}} \cos (\varphi) .
$$

In the new coordinate system the boundaries of the pressurized region, Eq. (5.16), become:

$$
\bar{a}^{+}=-\bar{a}^{-}=\pi / 2 .
$$

Substitution of Eq. (C.3), Eq. (C.4) and (C.5) into Eq. (C.1) gives:

$$
\hat{Q}_{Y, k}(Y)=Y \int_{-\pi / 2}^{\pi / 2}\left(\left(\bar{\eta}\left(P_{H}\right)\right)^{-1}\left(\bar{\rho}\left(P_{H}\right)\right)^{-2}\right) d \varphi .
$$

Substitution of Eq. (C.6) into Eq. (5.11) and introducing function $C(Y)$ gives:

$$
\hat{Q}_{Y}(Y)=Y C(Y),
$$

with:

$$
C(Y)=\int_{-\pi / 2}^{\pi / 2}\left(\left(\bar{\eta}\left(P_{H}\right)\right)^{-1}\left(\bar{\rho}\left(P_{H}\right)\right)^{-2}\right) d \varphi .
$$




\section{C.2 Dimensionless flux gradient on different grids}

In Table C.1 the numerically obtained flux gradient $\partial \hat{Q}_{Y} / \partial Y$ (See Eq. (5.10)) at the centreline $Y=0$ is given for three grid sizes, where $N_{x}$ is the number of grid points in $X$ direction. A domain $X \rightarrow[-2.5,1.5]$ and $Y \rightarrow[-2.0,2.0]$ is used with maximum grid sizes of 2049x2049 nodes for the circular case, 2049x4097 nodes for $D=0.1$ and 1025x8193 nodes for $D=0.01$. The number of grid points in $Y$ direction is scaled accordingly. Notice that for thinner inlet layers $H_{\text {oil }}$ and for larger $N$ values, the results show a larger difference between the different grids, as an accurate representation of the pressure and film thickness for these cases requires a very fine mesh.

\begin{tabular}{|c|c|c|c|c|c|c|}
\hline \multirow{2}{*}{$N$} & \multirow{2}{*}{$L$} & \multirow{2}{*}{$D$} & \multirow{2}{*}{$H_{\text {oil }} / H_{\text {cff }}$} & \multicolumn{3}{|c|}{$\partial \hat{Q}_{Y} / \partial Y$ at $Y=0$} \\
\cline { 5 - 7 } & & & & $N_{X}=513$ & $N_{X}=1025$ & $N_{X}=2049$ \\
\hline 20 & 2.5 & 1 & 1.00 & 0.8850 & 0.8845 & 0.8843 \\
\hline & & & 0.50 & 0.9119 & 0.9108 & 0.9107 \\
\hline & & & 0.25 & 0.9046 & 0.9158 & 0.9169 \\
\hline 20 & 5.0 & 1 & 1.00 & 0.4809 & 0.4806 & 0.4808 \\
\hline & & & 0.50 & 0.4742 & 0.4769 & 0.4767 \\
\hline & & & 0.25 & 0.4593 & 0.4694 & 0.4673 \\
\hline 20 & 10 & 1 & 1.00 & 0.2376 & 0.2374 & 0.2374 \\
\hline & & & 0.50 & 0.2315 & 0.2326 & 0.2325 \\
\hline & & & 0.25 & 0.2252 & 0.2265 & 0.2266 \\
\hline 100 & 2.5 & 1 & 1.00 & 0.7656 & 0.7639 & 0.7634 \\
\hline & & & 0.50 & 0.5933 & 0.5899 & 0.5898 \\
\hline & & & 0.25 & 0.5437 & 0.5448 & 0.5591 \\
\hline 100 & 5.0 & 1 & 1.00 & 0.4071 & 0.4060 & 0.4060 \\
\hline & & & 0.50 & 0.2954 & 0.3002 & 0.2998 \\
\hline & & & 0.25 & 0.2814 & 0.2641 & 0.2731 \\
\hline 100 & 10 & 1 & 1.00 & 0.1995 & 0.1989 & 0.1988 \\
\hline & & & 0.50 & 0.1513 & 0.1508 & 0.1505 \\
\hline & & & 0.25 & 0.1335 & 0.1326 & 0.1324 \\
\hline 1000 & 2.5 & 1 & 1.00 & 0.6179 & 0.5861 & 0.5821 \\
\hline & & & 0.50 & 0.2946 & 0.2911 & 0.2809 \\
\hline & & & 0.25 & 0.2672 & 0.2324 & 0.2484 \\
\hline 1000 & 5.0 & 1 & 1.00 & 0.3227 & 0.3115 & 0.3074 \\
\hline & & & 0.50 & 0.1501 & 0.1423 & 0.1499 \\
\hline & & & 0.25 & 0.1048 & 0.1150 & 0.1263 \\
\hline 1000 & 10 & 1 & 1.00 & 0.1574 & 0.1518 & 0.1504 \\
\hline & & & 0.50 & 0.0779 & 0.0771 & 0.0772 \\
\hline & & & 0.25 & 0.0676 & 0.0598 & 0.0646 \\
\hline & 10 & 0.1 & 1.00 & 0.7499 & 0.7473 & Not calcu. \\
\hline & & & 0.50 & 0.2233 & 0.2264 & Not calcu. \\
\hline & 10 & 0.01 & 1.00 & 1.2243 & 1.2231 & Not calcu. \\
\hline & & & 0.50 & 0.2793 & 0.2803 & Not calcu. \\
\hline & & & 0.25 & 0.2459 & 0.2557 & Not calcu. \\
\hline
\end{tabular}

Table C.1: $\partial \hat{Q}_{Y} / \partial Y$ at $Y=0$, for various values of $N, L$ and $D$ and grid size $N_{x}$. 


\section{C.3 Film thickness: experimental results}

In this section experimental results of the central film thickness decay in a circular as well as an elliptical contact at different velocities are presented; see Figure C.1 and Figure C.2, respectively. The properties of the lubricant and the solids are given in Table 5.2. The test conditions for the measurement series are given the figures and the captions.
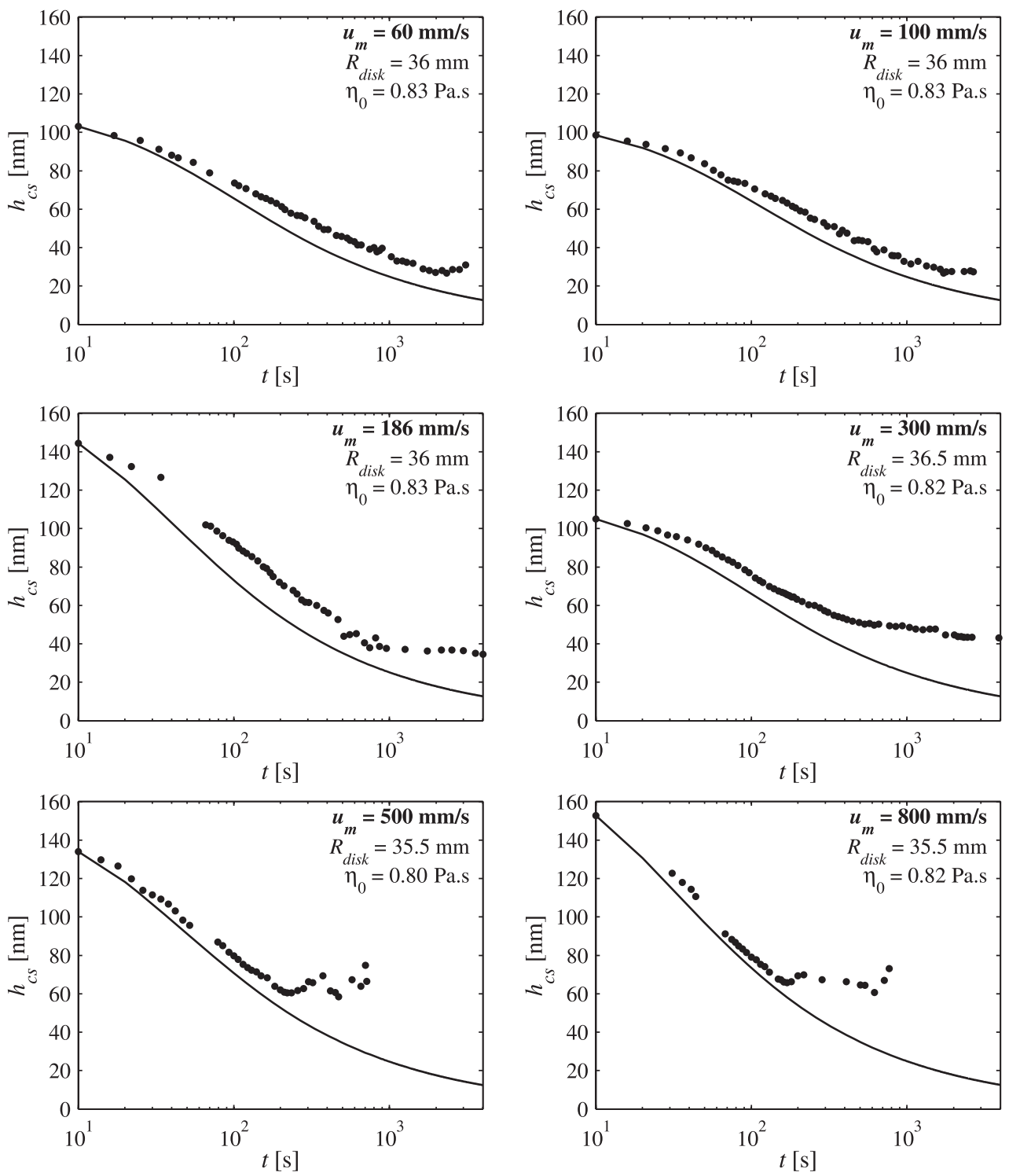

Figure C.1: Measured (dots) and predicted (solid lines) central film thickness. Circular starved contact at different velocities. $F=20 \mathrm{~N}, p_{h}=0.51 \mathrm{GPa}$, and $a=b=136 \mu \mathrm{m}$. 

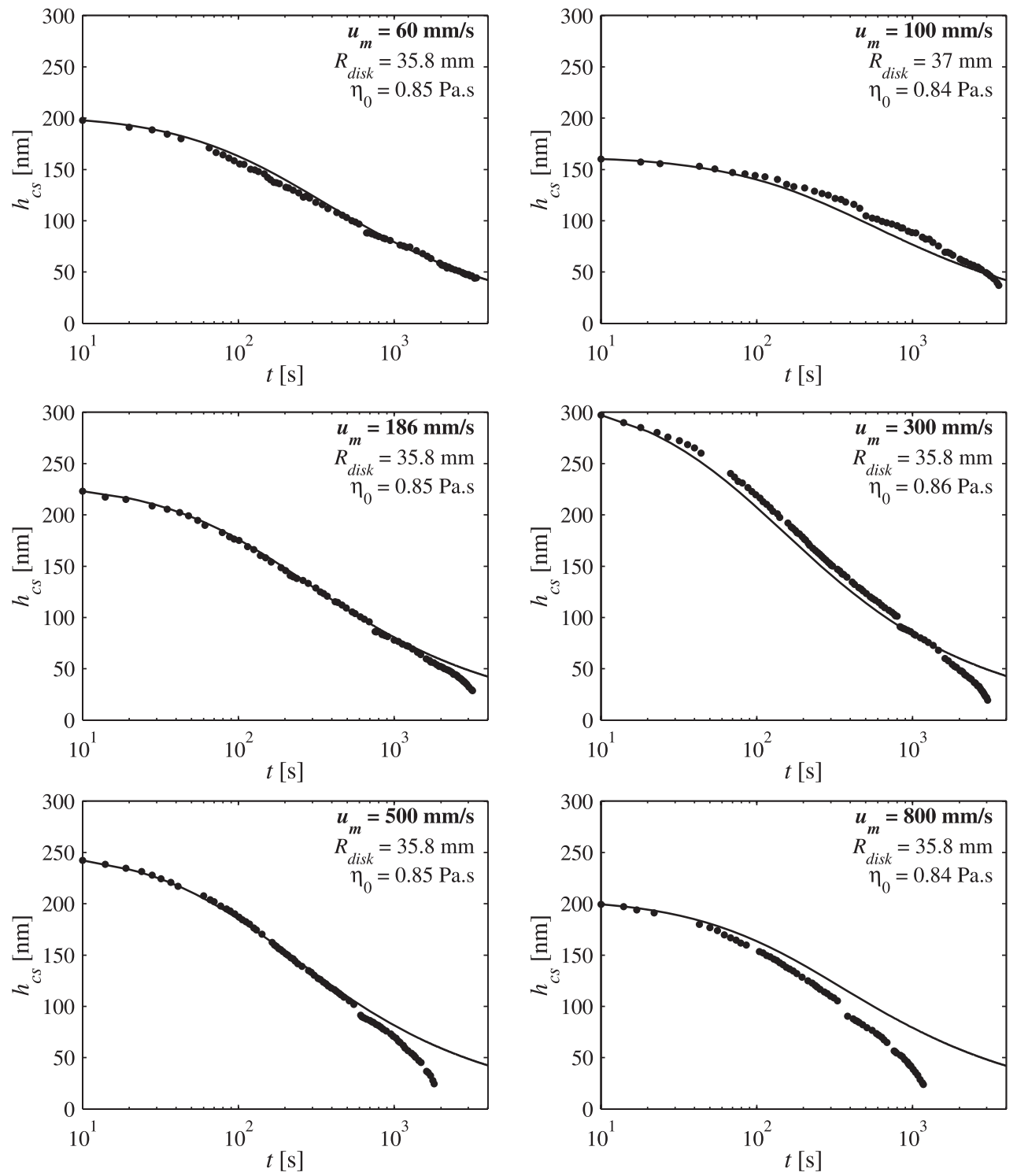

Figure C.2: Measured (dots) and predicted (solid lines) central film thickness. Elliptic starved contact at different velocities. $\kappa=0.27, \quad F=30 \mathrm{~N}, \quad p_{h}=0.33 \mathrm{GPa}, \quad a=108 \mu \mathrm{m}$, $b=398 \mu \mathrm{m}$. 


\section{C.4 Details implementation Chevalier/Damiens model}

In the layer thickness decay model presented by Damiens [19] the layer thickness at the inlet of the contact during the first overrolling $n=1$ is defined by: $h_{\text {oil }, 1}=\bar{\rho}_{c} h_{c f f}$. For the calculation of the results presented in Figure 5.16, the value of the parameter $\gamma$ as a function of $r_{n}$ is obtained by piecewise linear interpolation:

$$
\gamma=\left\{\begin{array}{lll}
\gamma_{1}+\left(\gamma_{2}-\gamma_{1}\right)\left(1-r_{n}\right) / 0.7 & \text { if } 0.3<r_{n} \leq 1.0 \\
\gamma_{2}+\left(\gamma_{3}-\gamma_{2}\right)\left(0.3-r_{n}\right) / 0.2 & \text { if } 0.1<r_{n} \leq 0.3 \\
\gamma_{3} & \text { if } 0<r_{n} \leq 0.1
\end{array}\right.
$$

where the values of $\gamma_{1}, \gamma_{2}$ and $\gamma_{3}$ are taken from the results presented by Damiens [19], see Table C.2.

\begin{tabular}{|c|c|c|c|c|c|}
\hline $\begin{array}{c}\text { Measurement } \\
\text { series }\end{array}$ & $M$ & $L$ & $\gamma_{1}$ & $\gamma_{2}$ & $\gamma_{3}$ \\
\hline $\mathbf{4}$ & 96 & 9.1 & 6.3 & 4.5 & 3.3 \\
\hline $\mathbf{5}$ & 41 & 9.4 & 5.8 & 4.3 & 3.3 \\
\hline $\mathbf{6}$ & 20 & 12 & 5.4 & 4.2 & 3.3 \\
\hline
\end{tabular}

Table C.2: Parameters $\gamma_{1}, \gamma_{2}$ and $\gamma_{3}$.

The film thickness decay model presented in Chapter 5 is a function of time, whereas Eq. (5.32) is a function of the number of overrollings. In order to compare the results of the models and the experimental results, a relation between $n$ and $t$ is defined. Considering the roller and disk experiment, the disk and rolling element (ball/roller) are overrolled at a different frequency:

$$
f r_{\text {disk }}=\frac{u_{m}}{2 \pi R_{\text {disk }}}, f r_{\text {roller }}=\frac{u_{m}}{2 \pi R_{x, \text { roller }}} .
$$

The number of overrollings $n$ is found by using the average frequency:

$$
n=\frac{1}{2}\left(f r_{\text {roller }}+f r_{\text {disk }}\right) t \text {. }
$$

Substitution of Eq. (C.10) and rearranging gives:

$$
t=\frac{4 \pi}{u_{m}} \frac{R_{\text {disk }} R_{x, \text { roller }}}{R_{\text {disk }}+R_{x, \text { roller }}} n .
$$




\section{Appendix D}

\section{D.1 Dimensionless flow rate}

In this section the equation for the dimensionless flow rate $\hat{Q}_{Y}$ is derived for the model presented in Chapter 6. The same scaling is used as in Chapter 5, see Eq. (5.7), with $p_{h}=\breve{p}_{h, i}, \quad a=\breve{a}_{i}, \quad b=\breve{b}_{i}$ and $c=\breve{c}_{i}$, and the scaling of $q_{y, i}$ and $q_{y, o}$ is similar to the scaling of $q_{y, k}$. The following ratios are defined:

$$
\phi_{a}=\breve{a}_{i} / \breve{a}_{o} \text {, and } \phi_{b}=\breve{b}_{i} / \breve{b}_{o} \text {. }
$$

The load is scaled according to Eq. (6.19). Substitution of $n_{r}=n_{c} / 2$ in Eq. (6.13) and scaling gives:

$$
\hat{Q}_{Y}(Y, \bar{t})=\frac{1}{4 \pi} \int_{0}^{2 \pi}\left(\hat{Q}_{Y, i}(Y, \bar{t}, \psi)+\hat{Q}_{Y, o}(Y, \bar{t}, \psi)\right) d \psi .
$$

Scaling of Eq. (6.14) gives:

$$
H(X, Y, \psi, \bar{t})=\frac{H_{o i l}(Y, \bar{t})}{\bar{\rho}(P)} .
$$

Substitution of Eq. (D.3) into Eq. (5.10) gives the dimensionless mass flow rate for a single contact $\hat{Q}_{Y, k}$. For a contact on the inner and the outer raceway it is, respectively:

$\hat{Q}_{Y, i}(Y, \bar{t}, \psi)=\int_{\bar{a}_{i}^{-}}^{\bar{a}_{i}^{+}}\left(-\bar{\rho}^{-2} \bar{\eta}^{-1} \frac{\partial P_{i}}{\partial Y}\right) d X$, with $\bar{\rho}=\bar{\rho}\left(P_{i}\right)$, and $\bar{\eta}=\bar{\eta}\left(P_{i}\right)$, and

$\hat{Q}_{Y, o}(Y, \bar{t}, \psi)=\int_{\bar{a}_{o}^{-}}^{\bar{a}_{o}^{+}}\left(-\bar{\rho}^{-2} \bar{\eta}^{-1} \frac{\partial P_{o}}{\partial Y}\right) d X$, with $\bar{\rho}=\bar{\rho}\left(P_{o}\right)$, and $\bar{\eta}=\bar{\eta}\left(P_{o}\right)$,

where the pressure distribution, Eq. (6.16), in dimensionless variables for a contact on the inner and the outer raceway is:

$$
\begin{aligned}
& P_{i}(X, Y, \psi)=\sqrt{\left(\bar{F}_{i}(\psi)\right)^{\frac{2}{3}}-X^{2}-Y^{2}} \text {, and } \\
& P_{o}(X, Y, \psi)=\phi_{a} \phi_{b} \sqrt{\left(\bar{F}_{o}(\psi)\right)^{\frac{2}{3}}-\left(\phi_{a} X\right)^{2}-\left(\phi_{b} Y\right)^{2}},
\end{aligned}
$$

respectively. The location of the boundary of the pressurized region in $X$, Eq. (6.17), in dimensionless variables for a contact on the inner and the outer raceway is: 


$$
\begin{aligned}
& \bar{a}_{i}^{+}=-\bar{a}_{i}^{-}=\sqrt{\left(\bar{F}_{i}(\psi)\right)^{\frac{2}{3}}-Y^{2}}, \text { and } \\
& \bar{a}_{o}^{+}=-\bar{a}_{o}^{-}=\frac{1}{\phi_{a}} \sqrt{\left(\bar{F}_{o}(\psi)\right)^{\frac{2}{3}}-\left(\phi_{b} Y\right)^{2}},
\end{aligned}
$$

respectively. Substitution of the derivative of Eq. (D.5) with respect to $Y$ into Eq. (D.4) gives:

$$
\begin{aligned}
& \hat{Q}_{Y, i}(Y, \psi)=\int_{\bar{a}_{i}^{-}}^{\bar{a}_{i}^{+}}\left(\bar{\rho}^{-2} \bar{\eta}^{-1} \frac{Y}{P_{i}}\right) d X, \text { and } \\
& \hat{Q}_{Y, o}(Y, \psi)=\phi_{a}^{2} \phi_{b}^{4} \int_{\bar{a}_{o}^{-}}^{\bar{a}_{o}^{+}}\left(\bar{\rho}^{-2} \bar{\eta}^{-1} \frac{Y}{P_{o}}\right) d X .
\end{aligned}
$$

Coordinate $\varphi$ is introduced and the following coordinate transformation is defined:

$$
X=\left\{\begin{array}{cc}
\sqrt{\left(\bar{F}_{i}(\psi)\right)^{\frac{2}{3}}-Y^{2}} \sin (\varphi) & \text { inner raceway contacts } \\
\frac{1}{\phi_{a}} \sqrt{\left(\bar{F}_{o}(\psi)\right)^{\frac{2}{3}}-\left(\phi_{b} Y\right)^{2}} \sin (\varphi) & \text { outer raceway contacts }
\end{array}\right.
$$

Differentiation of Eq. (D.8) with respect to $\varphi$ gives:

$$
d X=\left\{\begin{array}{cc}
\sqrt{\left(\bar{F}_{i}(\psi)\right)^{\frac{2}{3}}-Y^{2}} \cos (\varphi) d \varphi & \text { inner raceway contacts } \\
\frac{1}{\phi_{a}} \sqrt{\left(\bar{F}_{o}(\psi)\right)^{\frac{2}{3}}-\left(\phi_{b} Y\right)^{2}} \cos (\varphi) d \varphi & \text { outer raceway contacts }
\end{array}\right.
$$

Substitution of Eq. (D.8) into Eq. (D.5) gives:

$$
\begin{aligned}
& P_{i}(\varphi, Y, \psi)=\sqrt{\left(\bar{F}_{i}(\psi)\right)^{\frac{2}{3}}-Y^{2}} \cos (\varphi), \text { and } \\
& P_{o}(\varphi, Y, \psi)=\phi_{a} \phi_{b} \sqrt{\left(\bar{F}_{o}(\psi)\right)^{\frac{2}{3}}-\left(\phi_{b} Y\right)^{2}} \cos (\varphi) .
\end{aligned}
$$

In the new coordinate system the boundaries of the pressurized region, Eq. (D.6) become:

$$
\bar{a}_{i}^{+}=-\bar{a}_{i}^{-}=\pi / 2, \quad \text { and } \quad \bar{a}_{o}^{+}=-\bar{a}_{o}^{-}=\pi / 2 .
$$

Substitution of Eqs. (D.9), (D.10), and (D.11) into Eq. (D.7) gives:

$$
\begin{aligned}
& \hat{Q}_{Y, i}(Y, \psi)=\int_{-\pi / 2}^{\pi / 2}\left(\bar{\rho}^{-2} \bar{\eta}^{-1}\right) d \varphi, \text { and } \\
& \hat{Q}_{Y, o}(Y, \psi)=\phi_{b}^{3} \int_{-\pi / 2}^{\pi / 2}\left(\bar{\rho}^{-2} \bar{\eta}^{-1}\right) d \varphi .
\end{aligned}
$$

Substitution of Eq. (D.12) into Eq. (D.2) gives: 


$$
\hat{Q}_{Y}(Y)=Y C(Y),
$$

with:

$$
C(Y)=\frac{1}{4 \pi} \int_{0}^{2 \pi}\left(\int_{-\pi / 2}^{\pi / 2}\left(\left(\bar{\rho}\left(P_{i}\right)\right)^{-2}\left(\bar{\eta}\left(P_{i}\right)\right)^{-1}\right) d \varphi+\phi_{b}^{3} \int_{-\pi / 2}^{\pi / 2}\left(\left(\bar{\rho}\left(P_{o}\right)\right)^{-2}\left(\bar{\eta}\left(P_{o}\right)\right)^{-1}\right) d \varphi\right) d \psi
$$

\section{D.2 Hydraulic jump}

The dimensionless equation for the layer thickness is defined as given in Eq. (5.13), i.e.:

$$
\frac{\partial}{\partial Y}\left(\tilde{H}^{3} Q\right)+\frac{\partial \tilde{H}}{\partial \bar{t}}=0,
$$

where $\tilde{H}=\tilde{H}(Y, \bar{t})$. In this section $Q=Q(Y)$ is assumed to be constant in time, as is the case for the asymptotic film thickness model. Equation (D.15) can give multiple solutions for the layer thickness $\tilde{H}$ for a certain position $Y$. An example of such a multi-valued solution is shown in Figure D.1 (black line) in dimensional variables for one of the examples presented in Chapter 6, see Figure 6.7. Mathematically this solution is correct. However, this result violates the assumptions of lubrication theory, as the lubricant layer is no longer continuous in the thickness direction. Therefore, the multi-valued solution is converted into a single valued solution introducing a hydraulic jump (grey line) in the layer thickness. In the next sections the integral form of Eq. (D.15) is given and relations for the position and the velocity of the hydraulic jump are derived.

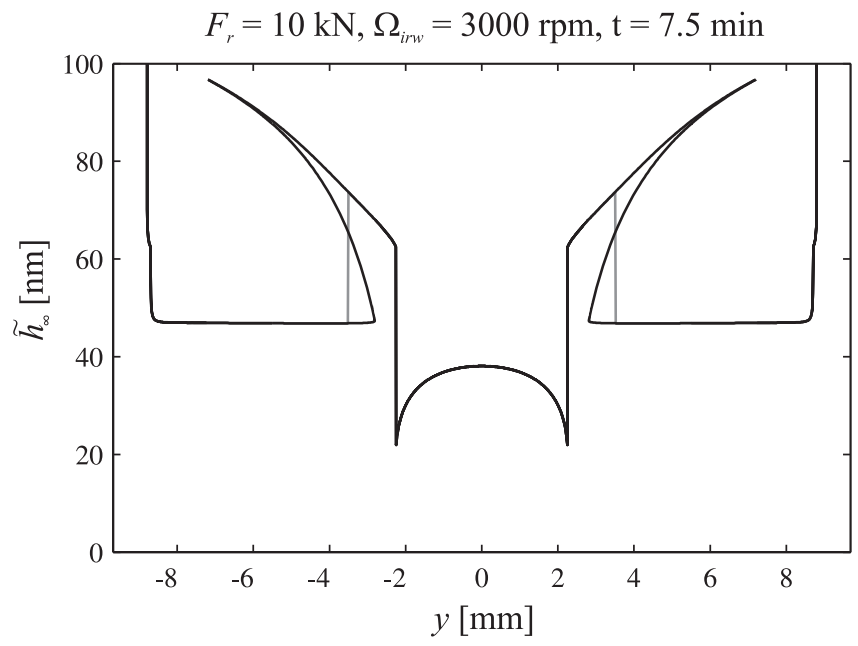

Figure D.1: Layer thickness distribution for one of the examples presented in Chapter 6, see Figure 6.7. A multi-valued solution is found. 


\section{D.2.1 Integral conservation form}

As the derivative with respect to $Y$ is unknown at the hydraulic jump, to find the position and the velocity of the jump Eq. (D.15) is written in the integral conservation form. This form does not contain any derivatives with respect to $Y$. It is obtained by integration of Eq. (D.15) over the domain $Y \in\left[Y_{-}(t) Y_{+}(t)\right]$ :

$$
\begin{aligned}
& \int_{Y_{-}(\bar{t})}^{Y_{+}(\bar{t})}\left(\frac{\partial}{\partial Y}\left(\tilde{H}^{3} Q\right)+\frac{\partial \tilde{H}}{\partial \bar{t}}\right) d Y=0 \Rightarrow \\
& \tilde{H}_{+}^{3} Q\left(Y_{+}\right)-\tilde{H}_{-}^{3} Q\left(Y_{-}\right)+\int_{Y_{-}(\bar{t})}^{Y_{+}(\bar{t})}\left(\frac{\partial \tilde{H}}{\partial \bar{t}}\right) d Y=0,
\end{aligned}
$$

where $\tilde{H}_{-}=\tilde{H}_{-}\left(Y_{-}(\bar{t}), \bar{t}\right)$ and $H_{+}=H_{+}\left(Y_{+}(\bar{t}), \bar{t}\right)$. Using the Leibniz integral rule Eq. (D.16) becomes:

$$
\tilde{H}_{+}^{3} Q\left(Y_{+}\right)-\tilde{H}_{-}^{3} Q\left(Y_{-}\right)+\frac{d}{d \bar{t}} \int_{Y_{-}(\bar{t})}^{Y_{+}(\bar{t})} \tilde{H}(Y, \bar{t}) d Y+\tilde{H}_{-} \frac{d Y_{-}}{d \bar{t}}-\tilde{H}_{+} \frac{d Y_{+}}{d \bar{t}}=0
$$

\section{D.2.2 Jump position}

Let the solution exhibit a hydraulic jump at $Y_{*}(\bar{t})$ in the domain $Y \in\left[Y_{-}(\bar{t}) Y_{+}(\bar{t})\right]$, see Figure D.2. Equation (D.15) is solved using the method of characteristics. The layer thickness at the boundaries $Y_{-}(\bar{t})$ and $Y_{+}(\bar{t})$ is:

$$
\tilde{H}_{-}\left(Y_{-}, \bar{t}\right)=\left(\frac{Q\left(Y_{0-}\right)}{Q\left(Y_{-}\right)}\right)^{\frac{1}{3}} \tilde{H}\left(Y_{0-}\right) \quad \text { and } \quad \tilde{H}_{+}\left(Y_{+}, \bar{t}\right)=\left(\frac{Q\left(Y_{0+}\right)}{Q\left(Y_{+}\right)}\right)^{\frac{1}{3}} \tilde{H}\left(Y_{0+}\right) \text {, }
$$

respectively, where $Y_{0-}$ and $Y_{0+}$ are the starting position of the characteristics on $Y$.

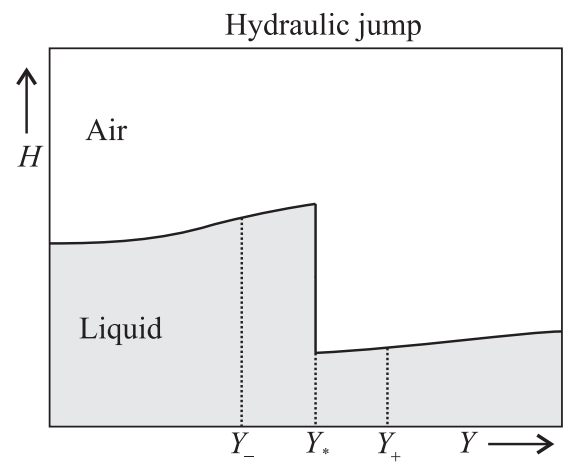

Figure D.2: Sketch layer thickness distribution. The solution exhibits a hydraulic jump at $Y_{*}$. 
When $Y_{-}(\bar{t})$ and $Y_{+}(\bar{t})$ follow the characteristic projections the following relations hold:

$$
\frac{d Y_{-}}{d \bar{t}}=3 \tilde{H}_{-}^{2} Q\left(Y_{-}\right) \quad \text { and } \quad \frac{d Y_{+}}{d \bar{t}}=3 \tilde{H}_{+}^{2} Q\left(Y_{+}\right) .
$$

Substitution of Eq. (D.18) and Eq. (D.19) into Eq. (D.17) gives:

$$
-2\left(\tilde{H}\left(Y_{0+}\right)\right)^{3} Q\left(Y_{0+}\right)+2\left(\tilde{H}\left(Y_{0-}\right)\right)^{3} Q\left(Y_{0-}\right)+\frac{d}{d \bar{t}} \int_{Y_{-}(\bar{t})}^{Y_{+}(\bar{t})} \tilde{H}(Y, \bar{t}) d Y=0 .
$$

Integration of Eq. (D.20) with respect to $\bar{t}$ gives:

$$
-2\left(\tilde{H}\left(Y_{0+}\right)\right)^{3} Q\left(Y_{0+}\right) \bar{t}+2\left(\tilde{H}\left(Y_{0-}\right)\right)^{3} Q\left(Y_{0-}\right) \bar{t}+\int_{Y_{-}(\bar{t})}^{Y_{+}(\bar{t})} \tilde{H}(Y, \bar{t}) d Y-\int_{Y_{0-}}^{Y_{0+}} \tilde{H}\left(Y_{0}\right) d Y_{0}=0
$$

In the limit $Y_{-}(\bar{t}) \uparrow Y_{*}(\bar{t})$ and $Y_{+}(\bar{t}) \downarrow Y_{*}(\bar{t})$ the third term of Eq. (D.21) is zero. Hence the starting position of the characteristics entering the jump at each side have to fulfill the following condition:

$$
-2\left(\tilde{H}\left(Y_{0+}\right)\right)^{3} Q\left(Y_{0+}\right) \bar{t}+2\left(\tilde{H}\left(Y_{0-}\right)\right)^{3} Q\left(Y_{0-}\right) \bar{t}-\int_{Y_{0-}}^{Y_{0+}} \tilde{H}\left(Y_{0}\right) d Y_{0}=0 .
$$

Using Eq. (D.22) the position of the jump is obtained indirectly. When a multi-valued solution is found for $Y_{0}$ at a position $Y$, Eq. (D.22) is used to determine which solution is valid. The minimum and maximum value of $Y_{0}$, i.e. $Y_{0_{-}}$and $Y_{0+}$ respectively, are substituted into Eq. (D.22). When the left hand side of Eq. (D.22) is smaller than zero, $Y_{0-}$ is used and when the left hand side of Eq. (D.22) is larger than zero, $Y_{0+}$ is used to calculate the layer thickness. For an initially uniform layer with thickness $\tilde{H}_{0}$ Eq. (D.22) becomes:

$$
2 \tilde{H}_{0}^{2} \bar{t}\left(Q\left(Y_{0-}\right)-Q\left(Y_{0+}\right)\right)-Y_{0+}+Y_{0-}=0
$$

\section{D.2.3 Jump velocity}

When $Y_{-}(\bar{t}) \uparrow Y_{*}(\bar{t})$ and $Y_{+}(\bar{t}) \downarrow Y_{*}(\bar{t})$ in the limit the third term of Eq. (D.17) is zero and Eq. (D.17) becomes:

$$
\tilde{H}_{+}^{3} Q\left(Y_{*}\right)-\tilde{H}_{-}^{3} Q\left(Y_{*}\right)+\tilde{H}_{-} \frac{d Y_{*}}{d \bar{t}}-\tilde{H}_{+} \frac{d Y_{*}}{d \bar{t}}=0
$$

Equation (D.24) can be written as:

$$
\frac{d Y_{*}}{d \bar{t}}=Q\left(Y_{*}\right)\left(\tilde{H}_{-}^{2}+\tilde{H}_{+} \tilde{H}_{-}+\tilde{H}_{+}^{2}\right),
$$

which is the Rankine-Hugoniot relation for Eq. (D.15) representing the velocity of the hydraulic jump. 


\section{D.3 Bearing properties}

In Table D.1 values of the relevant parameters for a radial ball bearing (209) and a spherical roller bearing (22317) are given. The values of the parameters until $P_{d}$ have been deduced from Harris [36]. The reader is referred to the nomenclature for the equations that have been used to calculate the other parameters.

\begin{tabular}{|c|c|c|c|c|}
\hline \multicolumn{5}{|l|}{ Properties of the bearings } \\
\hline & & $\begin{array}{l}\text { Deep groove } \\
\text { ball bearing } \\
209\end{array}$ & $\begin{array}{l}\text { Spherical } \\
\text { roller bearing } \\
22317\end{array}$ & \\
\hline Number of rolling elements & $n_{r}$ & 9 & 14 & - \\
\hline Mass rolling element & $m_{r}$ & $8.37 \cdot 10^{-3}$ & $7.93 \cdot 10^{-2}$ & $\mathrm{~kg}$ \\
\hline \multirow{2}{*}{$\begin{array}{l}\text { Radii of curvature rolling } \\
\text { element }\end{array}$} & $R_{x, \text { rol }}$ & $6.35 \cdot 10^{-3}$ & $12.50 \cdot 10^{-3}$ & $\mathrm{~m}$ \\
\hline & $R_{y, \text { rol }}$ & $6.35 \cdot 10^{-3}$ & $79.96 \cdot 10^{-3}$ & $\mathrm{~m}$ \\
\hline \multirow{2}{*}{$\begin{array}{l}\text { Radii of curvature inner } \\
\text { raceway }\end{array}$} & $R_{x, i r w}$ & $26.15 \cdot 10^{-3}$ & $55.33 \cdot 10^{-3}$ & $\mathrm{~m}$ \\
\hline & $R_{y, i r w}$ & $-6.60 \cdot 10^{-3}$ & $-81.59 \cdot 10^{-3}$ & $\mathrm{~m}$ \\
\hline \multirow{2}{*}{$\begin{array}{l}\text { Radii of curvature outer } \\
\text { raceway }\end{array}$} & $R_{x, \text { orw }}$ & $-38.9 \cdot 10^{-3}$ & $-79.78 \cdot 10^{-3}$ & $\mathrm{~m}$ \\
\hline & $R_{y, \text { orw }}$ & $-6.60 \cdot 10^{-3}$ & $-81.59 \cdot 10^{-3}$ & $\mathrm{~m}$ \\
\hline Reduced modulus of Elas. & $E^{\prime}$ & $227 \cdot 10^{9}$ & $227 \cdot 10^{9}$ & $\mathrm{~Pa}$ \\
\hline Diametral clearance & $P_{d}$ & 0.102 & 0.0152 & $\mathrm{~mm}$ \\
\hline Track length & $l_{t}$ & 0.767 & 1.95 & $\mathrm{~m}$ \\
\hline \multirow{2}{*}{$\begin{array}{l}\text { Ratios contact size } \\
\text { inner/outer raceway }\end{array}$} & $\phi_{a}$ & 0.810 & 0.822 & - \\
\hline & $\phi_{b}$ & 1.031 & 1.018 & - \\
\hline Load defection factor & $K_{n}$ & $1.17 \cdot 10^{10}$ & $4.29 \cdot 10^{10}$ & $\mathrm{~N} / \mathrm{m}^{1.5}$ \\
\hline \multicolumn{5}{|l|}{ Inner raceway contacts } \\
\hline Ellipticity ratio & $\kappa$ & 0.108 & 0.0250 & - \\
\hline Elliptic integral first kind & $\mathcal{K}$ & 3.622 & 5.077 & - \\
\hline Elliptic integral sec. kind & $\mathcal{E}$ & 1.018 & 1.0014 & - \\
\hline Reduced radius of curv. & $R$ & $4.96 \cdot 10^{-3}$ & $1.02 \cdot 10^{-2}$ & $\mathrm{~m}$ \\
\hline Load defection factor & $K_{i}$ & $3.22 \cdot 10^{10}$ & $1.19 \cdot 10^{11}$ & $\mathrm{~N} / \mathrm{m}^{1.5}$ \\
\hline \multicolumn{5}{|l|}{ Outer raceway contacts } \\
\hline Ellipticity ratio & $\kappa$ & 0.137 & 0.0309 & - \\
\hline Elliptic integral first kind & $\mathcal{K}$ & 3.38 & 4.863 & - \\
\hline Elliptic integral sec. kind & $\mathcal{E}$ & 1.027 & 1.0021 & - \\
\hline Reduced radius of curv. & $R$ & $7.26 \cdot 10^{-3}$ & $1.48 \cdot 10^{-2}$ & $\mathrm{~m}$ \\
\hline Load defection factor & $K_{o}$ & $3.41 \cdot 10^{10}$ & $1.24 \cdot 10^{11}$ & $\mathrm{~N} / \mathrm{m}^{1.5}$ \\
\hline
\end{tabular}

Table D.1: Properties of the single row deep-groove ball bearing 209 and spherical roller bearing 22317. 


\section{ACKNOWLEDGEMENTS}

During the course of the last years many people have contributed in various ways to the work presented in this thesis.

This research project was supported by SKF Engineering and Research Centre in Nieuwegein. I gratefully acknowledge this support.

I am most grateful to my mentors, Kees Venner from the University of Twente, and Piet Lugt from SKF ERC for their support, guidance and enthusiasm. I am also grateful to Harry Hoeijmakers for his scientific support and for the careful reading of the manuscript.

From SKF ERC I would like to thank John Tripp and George Popovici for their interest and helpful suggestions and Jos Holsnijders, Hans de Ruig, Ralph Meeuwenoord for their help in the experiments. During the research project I had the opportunity to work at SKF Nieuwegein for two months. I would like to thank all the people at SKF ERC that made me feel welcome.

I would like to thank the members of the Engineering Fluid Dynamics group of the Faculty of Engineering Technology of the University of Twente for their cooperation and support and for creating a very pleasant work atmosphere; professor Harry Hoeijmakers, scientific staff: Koen van Andel, Rob Hagmeijer, Mico Hirschberg, Niels Kruyt and Edwin van der Weide, my (former) roommates: Peter van Dijk, Sietse Jongsma, Arjen Koop, George Popovici, Hein de Vries, fellow PhD students: Dirk van Eijkeren, Ellen van Emden, Remco Habing, Jacco Hospers, Rutger IJzermans, Philip Kelleners, Ryan Sidin, Jesse Slot, Arie Verhoeff and Remko Westra. Support staff: Wouter den Breeijen, Anjenet Mettivier-Meyer and Herman Stobbe, and further thanks goes to all the MSc students.

I would like to express my gratitude to my friends and family for their support and friendship over the years. I would like to thank my parents Theo and Truus and my brother Arno and sister Nanda for their interest and encouragement during my life. Finally I thank Luan for her love, laughter and support.

M.T. van Zoelen

Enschede, September 2009 


\title{
About THE AUThor
}

\author{
August 27th, $1981 \quad$ : $\quad$ Born in Apeldoorn, the Netherlands. \\ 1993 - 1999 : VWO (pre-university education), \\ "De Heemgaard”, Apeldoorn, the Netherlands. \\ 1999 - $2004 \quad$ : Study Mechanical Engineering, \\ University of Twente, the Netherlands. \\ Specialisation: Applied Mechanics. \\ Master's thesis: Modeling of tire/road contact and tire \\ vibrations. \\ 2003 \\ : Internship at the High Pressure Waterjet Laboratory, \\ Missouri University of Science and Technology, Rolla, USA. \\ Subject: Mechatronics: design and implementation of software \\ and hardware for an X-Y positioning system. \\ 2004 - 2005 : Research employee, University of Twente, the Netherlands, \\ Department of Engineering Fluid Dynamics. \\ Project: Free surface thin layer flow and centrifugal effects on \\ bearing raceways, sponsored by SKF. \\ 2006 \\ : Mechnical engineer, Witteveen+Bos Consulting Engineers \\ b.v., Deventer, the Netherlands. \\ Job description: Design of mechanical and flow systems in \\ the field of civil engineering. \\ 2007 - 2009 : Ph.D. student, University of Twente, the Netherlands, \\ Department of Engineering Fluid Dynamics.
}

\section{Article publications}

van Zoelen, M. T., Venner, C. H. and Lugt, P. M. (2009), "Free Surface Thin Layer Flow in Bearings Induced by Centrifugal Effects,” STLE Tribology Transactions, Taylor \& Francis, in press.

van Zoelen, M. T., Venner, C. H. and Lugt, P. M. (2009), "Prediction of Film Thickness Decay in Starved EHL Contacts Using a Thin Layer Flow Model,” Proceedings of ImechE; Part J., Journal of Engineering Tribology, vol. 223, pp. 541-552.

van Zoelen, M. T., Venner, C. H. and Lugt, P. M. (2008), "Free Surface Thin Layer Flow on Bearing Raceways, ” ASME Journal of Tribology, vol. 130, no. 2, pp. 021802. 


\section{Awards}

Received the "Dowson Prize" at the $35^{\text {th }}$ Leeds-Lyon Symposium on Tribology, Leeds, UK, 9-12 September 2008. (Best paper and presentation at the Symposium by a registered research student, or someone within 3 years of graduation from a research degree).

\section{Conferences}

van Zoelen, M. T., Venner, C. H. and Lugt, P. M. (2009), "Free Surface Thin Layer Flow Modeling of Contact Pressure Induced Lubricant Migration in Rolling Element Bearings," STLE Annual Meeting, May 17-21, 2009, Lake Buena Vista, FL, USA, oral presentation.

van Zoelen, M. T., Venner, C. H. and Lugt, P. M. (2009), "Prediction of Film Thickness Decay in Starved EHL Contacts Using a Thin Layer Flow Model,” JMBC day, January 2009, Eindhoven University of Technology, the Netherlands, poster contribution.

van Zoelen, M. T., Venner, C. H. and Lugt, P. M. (2009), “Thin Layer Flow in Rolling Element Bearings,” JMBC day, January 2009, Eindhoven University of Technology, the Netherlands, oral presentation.

van Zoelen, M. T., Venner, C. H. and Lugt, P. M. (2008), "Prediction of Film Thickness Decay in Starved EHL Contacts Using a Thin Layer Flow Model," $35^{\text {th }}$ Leeds-Lyon Symposium on Tribology, September 9-12, 2008, Leeds, UK, oral presentation.

van Zoelen, M. T., Venner, C. H. and Lugt, P. M. (2008), "Centrifugal Effects and Free Surface Thin Layer Flow in Roller Bearings,” STLE Annual Meeting, May 18-22, 2008, Cleveland, $\mathrm{OH}$, USA, oral presentation.

van Zoelen, M. T., Venner, C. H. and Lugt, P. M. (2008), “Free Surface Thin Layer Flow on Bearing raceways," JMBC day, January 2008, Delft University of Technology, the Netherlands, poster contribution. 